\title{
PAVLI AEGI:
}

NETAE PHARMACA SIM=

$$
\begin{aligned}
& \text { plicia, Othone Brunfelfio } \\
& \text { interprete. }
\end{aligned}
$$

IDEM DE RATIONE VICTVS

Gulielmo Copo Bafilienfi

$$
\text { interprete. }
$$

\section{Pre - Livin RSI64}
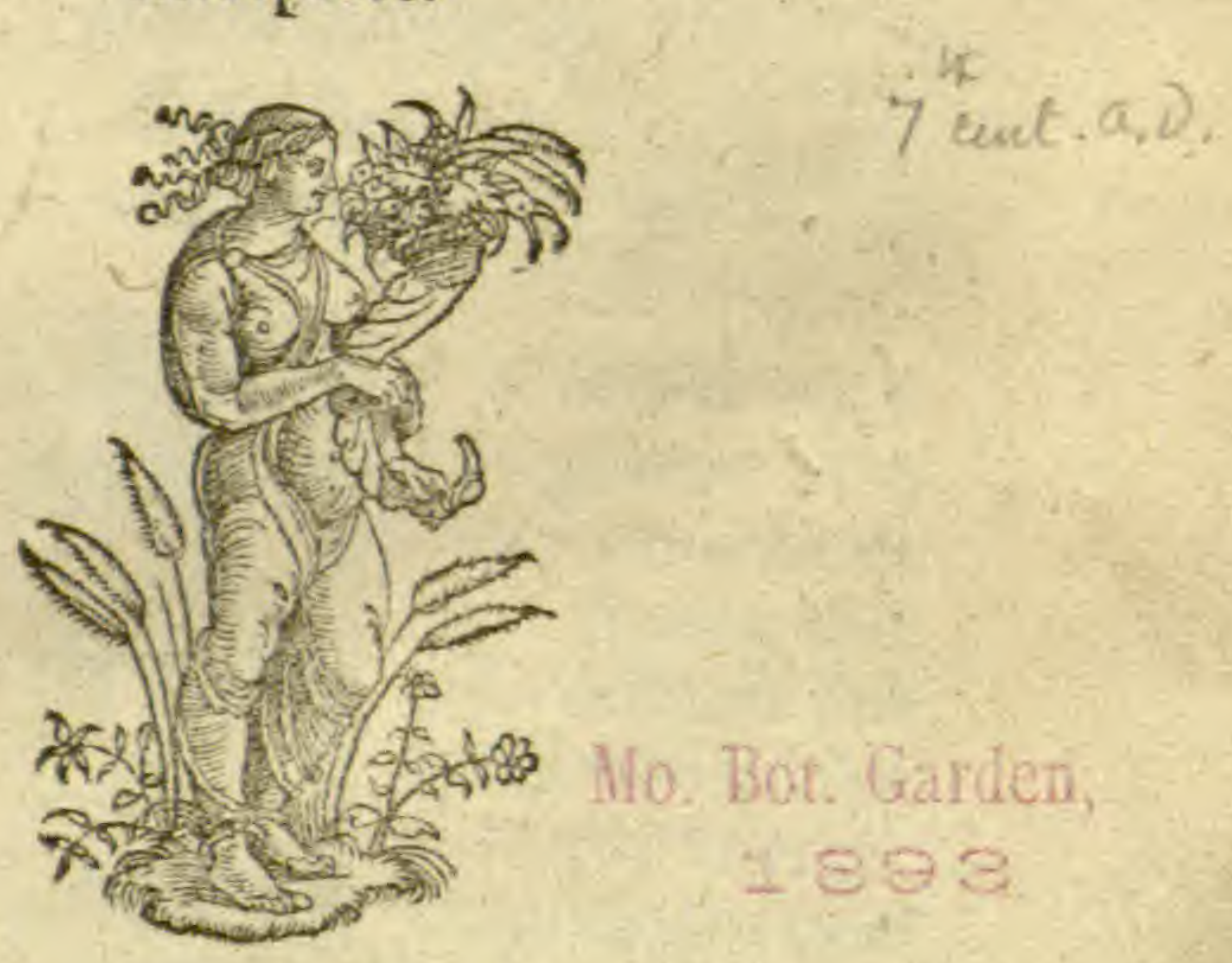

IN PAVLVM AEGINETAM DE

Simplcibus, tuxta ac de Ratione $V u t$ tus, Index tum utilis, twam necc]jsitus.

$$
\begin{aligned}
& \text { is } 31 \text { gusitt. } \\
& \text { Niralaus Roumprom }
\end{aligned}
$$




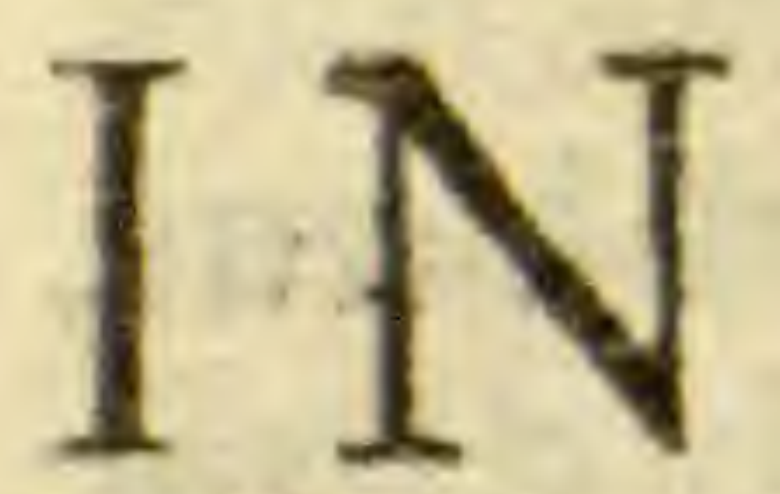

ET GENEROSIS

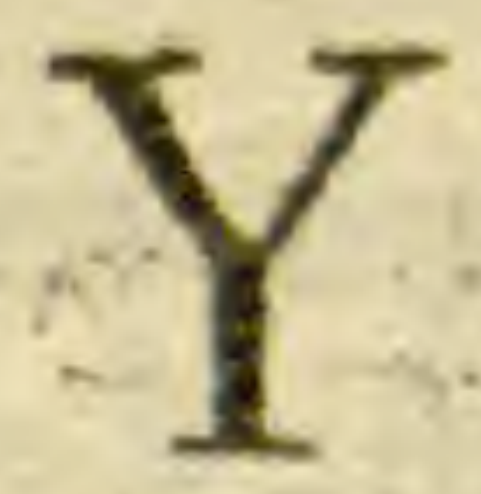

DOMHNIS., D.

Othoni \& Volphgango Conitis

bus à Solms, duarum ìnfiguti=

um Ecclefiarum, Argenti=

nenfis \& Colonienfis,

Canonicis . D. fuis

obfercuandiffimis

Otho Branfel=

fius S. D.

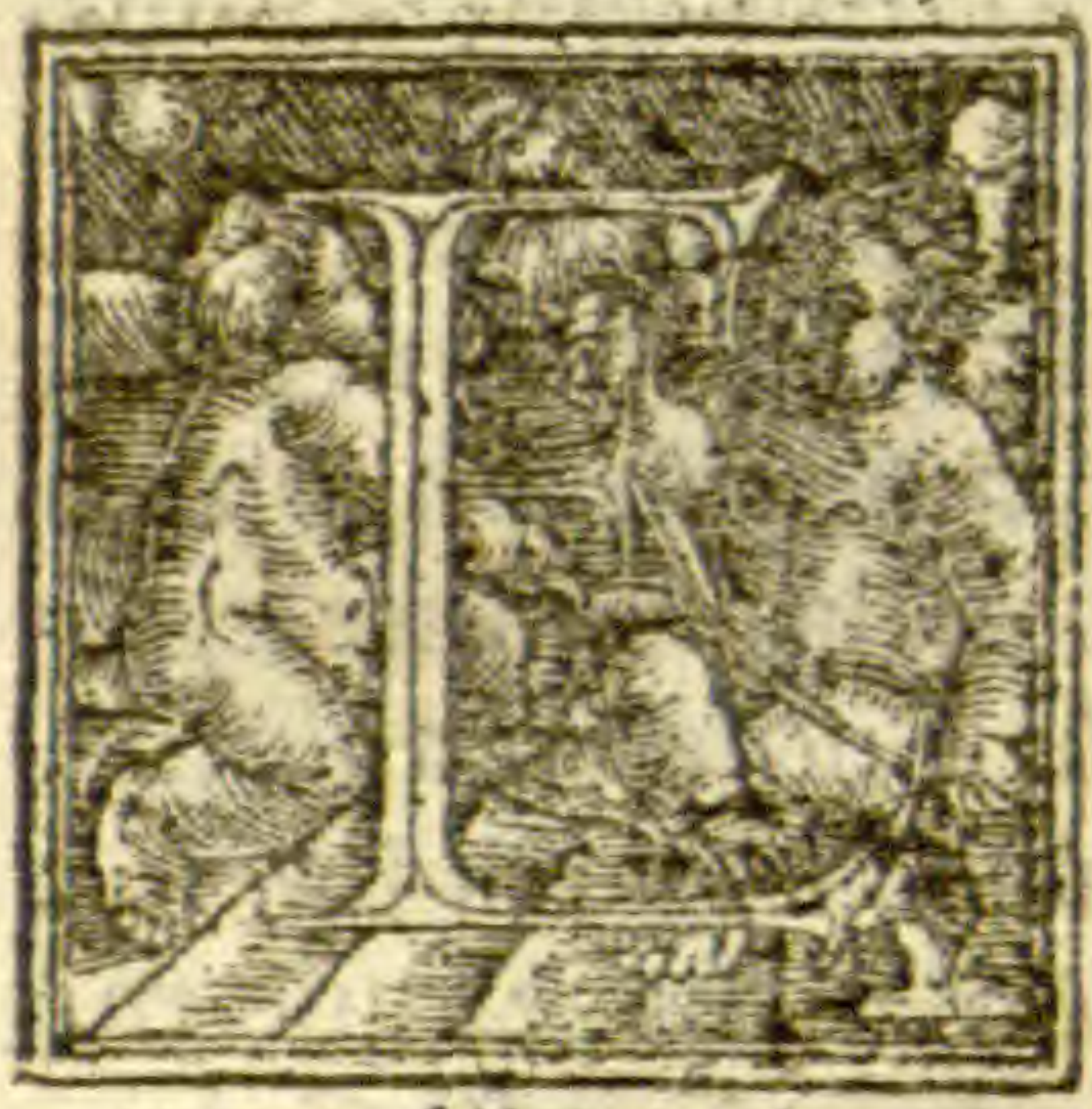

N MITTO PAV

li-Aeginetx, De fimplicibus nredi cinis libellü, qué inffu ueftrarü Cel fitudinum tietter dü nuper fulcep?.

Quanquam noir ea grandiloquencia, eogí dicendi lepore redditum, quo ego eum authorem Velt. Celf. deptredicaui, antequã noffem qui

$$
\text { A } 2 \text { dem }
$$




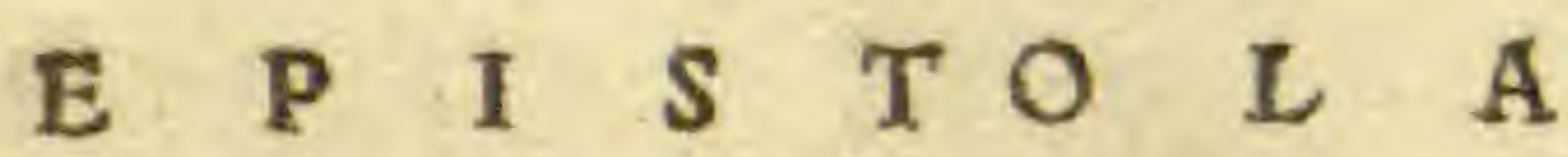

dem, quale fuiffem exéplare habiturus, \& ueftre fortaffe Celfituditnes fibi de me pollicebantur. In cauffa fuit ualetudo illa mea, in qua tan immenfis, atq, innume: ris officijs me profecute, funt Celf.V. De= inde exemplare morofum, ac penè prodi giofe depranatum, quibus factum eft, ut uotorum neutiquam compos fieri potue rim, omniać in irritum ceciderint. $\mathrm{Co}=$ gor enim uel inuitus hicincómoda prx= fari mea, non tam ut lugeam meas fortu= nas, quàm ut beneuolentiâmihi captem apud Veft. Celfitud, ne quod impoten= tix, \& imbecillitatis fuit, ignauixpotius, atqgofcitantia imputetur. Defpeream, fi maiori anxietate in omni uita, praf fus unquam fuerim. Siquidem urgebat hinc Typographus, qui, cum impende= rent nundinæ, carthas aliquot ante rgria tudinem coeptas, fibi nolebar abfolui, Ithinc negocium faciebat codex ouv $\gamma_{\mu \alpha \alpha}$ $\tau$ íd'rs, mendofus $8 \mathrm{z}$ mancus, ex quo me exericare non ualebam. Er nifi Galeni Streliquorum Gracorum adiutus com= mentarins fuiffem, ex quibus mutuatus eft fua Aegineta, nonnunquam ad uers

bum, 


\section{N V N C V P A T O R I A:}

bum, interdum fententiam ex fententia, fateor ingente, fterilem meoperam ef = featurum fuiffe, nec multum momenti fuiffe confecutum. Omitto quod fre= quenter Galeno atq Diafcoridę pugnan tia habebat, alijs quoģ fubinde nomen= claturis defignabat. Commata aut 1nulla aut ea prapoftera habebat. Preter infinis taseclypfes, \& id genus uitiliginum, qui= bus deprauatilibri funt confpurcati.Quę quantü mutent de fententia, quantas $\partial \mu \Phi i \beta O \lambda \mid \alpha$ cient, non eft quod dicam, cum me multo etiam doctioribus impo nant. Teftis mihi Iacobus Bedorortus, uir Græce quoqg lingux abfolute doctus. Queritur idem $\& 2 D$. Allanus Torinus, ut audio, Bafiliếfis medicus, uiralioqui do= Ctiflimus, qui nunc totum Paulum in $\mathrm{ma}$ nibus habet, cuius tralationem, ut fem= per optaui, extare, ita inter facrum nunc mihi conftituto \& faxum, Veftraru Celf. promiffis fatisfaciendú fuit, ni aut impref fori, uiro lóge opt.cui \& ipfi fide nos ob= Atrinxeramus, detriméto effe, aut a pudC. Veft. futilis reperiri uoluiffem . Erit itaq́ Veft.Celf.hremea detrimenta, trutina: 


\section{E $P$ I $S$ T $T$ O}

nare, $\&$ non tam quid ipfe receperim, fed quid tŭ uires bene permiferint, expendes $r e$, meminiffeç illius, quod in prouerbio eft, Canis feftinans, ceecos catulos parit. Decuiffet \& Celf. ueftræ, claras imagines hic celebrare, fed qua mihi obftitit in cu= dendo fors atç̣ feftinátia eadé factú eft, ut

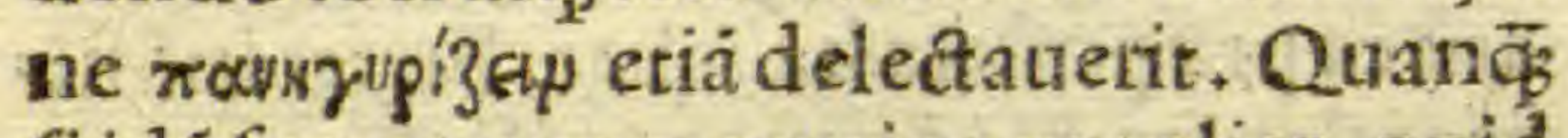
fiidé fucata quoqg oratione moliar, quid aliud quàm ebur atraméto candefaceré, aut foli lumé, quod dicitur, inferté? Qux enim infignes uirtutes, Cell.V.non pluri mum exornant: Et quam non laudê con fecurus eft, uéftra tam cafta, pia, \&liberali educatione, doetifs. preceptor uefter, $M$. Ioannes à Daurnheymic Erit ubi hrc fuis agenturlocis. Nunc penfum hocmeum beinigne fufcipite, fortunasqümeas mecŭ boni confulite \& ualete. Argentorati Cas lend.Septemb. An. à Chrifto noftro fertuatore nato, Millefimo quin gentefimo trigefimo:

primo.

$$
93
$$




\section{IN PAVLVM A E G I N E T A M D E}

Simplicibus, iuxtd ac de Ratione

V:ctus, Indexcum utilis

tum nece]Jarius.

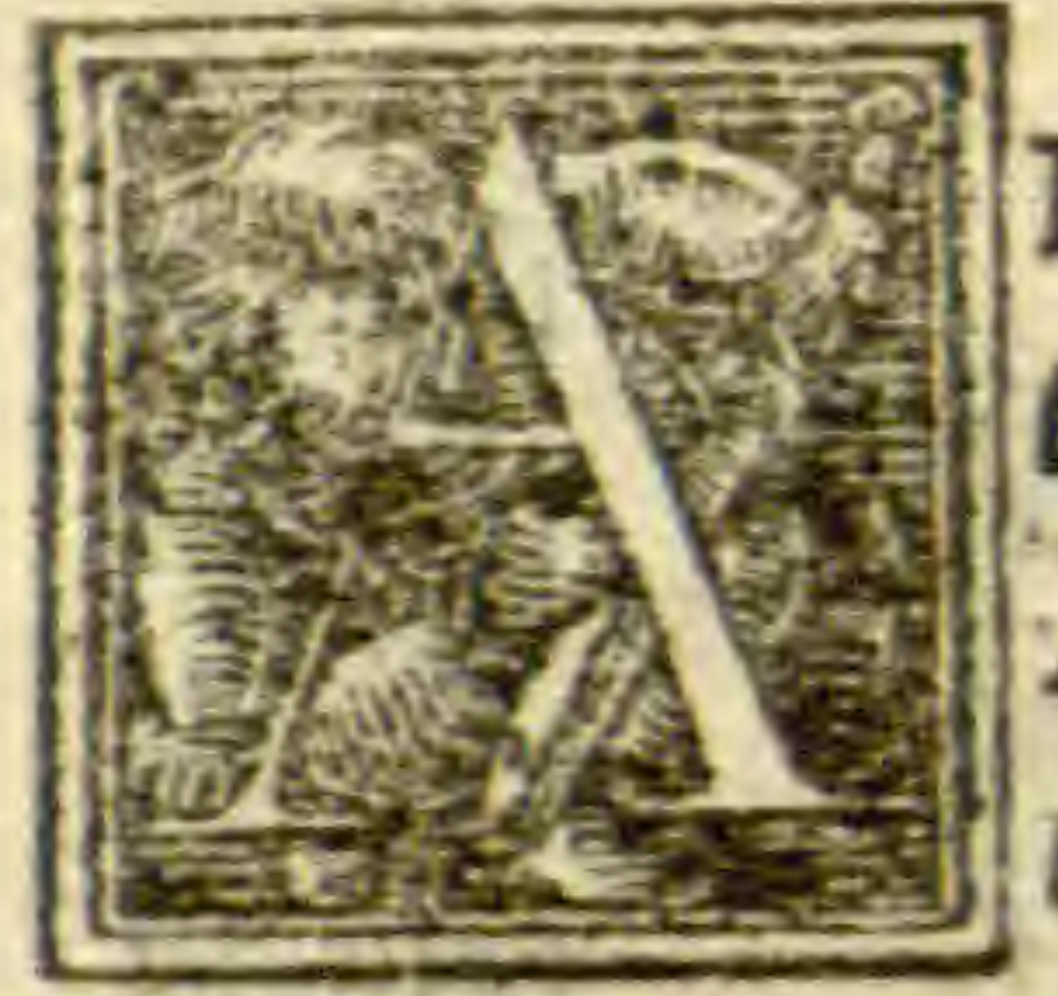

Acallis

Acatist

Aculiphe

Achantus

Achantion

Achantaleuce

Achanta Aegyptia

Acorus

Aciros

Arides

Afte

Acbids

Accrbum uinum

Acetam

Adarce
$\mathrm{B} \mathrm{RO}=$ Adianton

tanum iv Adeps

Abfinthi Adepsfuillus

11. Adeps Caprarum

3. Adeps Anferinus

3. Adeps Gallindceor $\vec{u} \sigma_{3}$.

4. Adeps Leonum

4. Adepsurfinus

4. Adcps uulpinus.

4. Adeps uiperce

4 Adeps mafculipeco=

4. ris

4. Adepsuetus

4. Aetonichon

4. Acgylops

11. Aegyros

83. Aetzale

5i. Acthiops

2. Acra
63.

$\sigma_{3 *}$

$\sigma_{3} \cdot$

$\sigma_{30}$

63.

63 .

2.

2.

20

2.

2.

3.

A 4 Acs 


\section{N D E X.}

Acs

Agallochum

Agaricum

Agcranton

Agnuscaftus

Agroftis

Agreftis paftinace

Aizoons

Althes

Alimum

Alcad

Alcyonia

Aloé

Alline.

Alypoit

Aly) Jon

filicas

Alauda

Amaracus

Amarantus

Aimbrofia

Ami

Amianton

Ammos

Amoniacum

Amorge

Amures
69: Ampcloprafum ibidem?

1. Ampclos agria

$7+$

ibidem

1. Amygdala dulcia ibidem

x. Amylum

1. Amomim

ibidem

ibidem.

78 .

2. Anchufe omnes

I*

5. Anagallis

5. Anagyris

7:

ibidem

5. Andrachne

5. Andrafiamion

ibidem

5. Androfartes

5. Anemone

o. Anetbum

6. Anthylits

74. Anofum

36. Anturbinum

6. Andrrbinon

ibidem, Anonis

8.

ibidem

ibtdem

ibidem

ibidem

ibidem

tbidem

ibidem

ibidem

ibidem. Animalia nolatilia 78 .

ibidem. Aparine

8.

ibıdems

ibidem

ibidem. Apocynon

11 .

ibidem, Aphace

ibidem. Aphenitron

ibidem, Aplorontron
$11+$

ibidem

Apoum 
I $\mathrm{A}$ D $\mathrm{D}$.

Apium

Aqua maris

Araneus

Argemone

Arifaron

Ariftolochid

Arceuthos

Arción

Arctiunt

Armenidcum

Arndibo

Arrogloffum

Aron

Arfenicum

Artimefice

Artos

Ajarum

Af bole

Aftalubotis

Afclepids

Afcyrron

A podatos

a)paragus

ajplenion

a) taphis

dfter atticus

affragulus 7z. Aphaltus

25. apphodelus

9. atherd

ibidem atbard

ibidem atractilis

ibidem atramentũfcriptor ${ }^{2} 5^{\circ}$

ibidem duriculamuris

Yo

ibidem aures

ibidem

abidem

ibidem

toidem

10.

ibidem

tbidem

ibidem

ibidem

tbidem

ibidem

ibidem

ibidem

ibidem

ibidem

ibidem

i1.

ibidem

ibidem Brathi

toident

ibidemt

सृ०

26

17. $\$ \sigma:$

B

D Alamus Myrepfica BBacharuls 12." (t)

Bdialufium ibidena

Balfamon

ibident ibidem

Battis

ibident

Batrachion ibtdent

Batrarchi

ibident

tbident

Betonica

$13^{2}$

Bechium

ibsdeat

Beta

$72^{6}$

Bitumen

$1 \mathrm{t}^{2}$

Blitum

ㄱis. 728

Blicorumlaetucdrū $73^{*}$

Blatte piftrinarice $60^{\circ}$

Botrys

$13^{3}$

135

Britanmed 


\section{$I \quad \mathbb{N} D \quad E \quad X$.}

Britdnnica 14 . CalamiCortes 28.

Bromos

ibidem

Camper

ibidem

Bryon Sphlanchnon 14.

Cantharides

Bryon Thalaßion 14. Cannalis

ibidem

Brapica

$72 \cdot 37$.

Bromus

75. Capnos

ibideme

Eraßicamarina

Bulbus

37. Capparis

13. Cardamomum

Bulbusuomitorius

13.

Bubonion

13. 11 .

Bugloffum

Bunion

13.

ibidem

Bupreftis

Butyrum

tbidem

ibidem

Buphtbalmon

Bunias

tbidem

73:

Bulbi

73.

Bucnia

32 .

\section{C}

Ancanus

Canchamum -28 .

cadmie

ibidem

Cacalia

ibtdem

Calamagroftis ibidem

Calaminthe

ibidem

Calamus aromaticus 28 .

Calamus phragmites 28 .

Canopon is.

Caris

29.

ibidem

Cancrifluniales ibidem Cancrimarini - ibidem

caron

ibidest

carpefium

Caria

ibidem

ibidem

Caryopbytlon 30.

Caffamon

ibidem:

caßsia

Caucalis

ibidem

ibldem

Capita Menidum

32

Capita Smaridum

Caput Muris

31.

Caput Milui

$i b t d c m$

CaputLacerte ibzdem

Capparis 73. Carice 76.

Caftanes 78 .

Caprehirci

79.

Caprinumlac.

81 .

Cafeus 


\section{N D E X X}

\section{Cafeus}

Capito

Cartiagineus

Capilus ueneris

Crrieslignorum

Cenchros

Cedrus

Centaureum

Centaureum minus

Ceratonits

Cecis

Cepca

Cera

Cepa

Cerafia

Cerut

Cerebrum

Cerebrumleporis

Cerebrum galli

Cerebrum Cameli

Cerebrummuftele

Cerebrum Chelido

Ccrebrun Ouillum

Chalbane

Cbalcitis

Cablcanthos

Chalcos
82;. Chondrilld

82. Chondros

83. Chrifocolla

3. Chrifocome

59. Chichorium

30. Cici

30. Cimnabaris

30.

30.

31.

31 .

32.

32.

74.

77.

49.

80.

19.

19.

19.

19.

19.

19.

69

69.

69.

69.

circed

Ctfferis

Ciftos

Ctffos

Citrium

Cinofbatus

Cicutd

Cicera

Cicere

Citreimali

Clcmatitis

Cnesus
Cinndmomum

Ciborum facultates

Clemattitis altera

Clenopodion

69.

70.

$70 \%$

70*

$33^{\circ}$

32.

$32 *$

$32^{\circ}$

320

$320^{\circ}$

32.

32.

$33^{\circ}$

38 .

39.

71.

75.

$75^{\circ}$

78.

33.

33.

33.

$33^{\circ}$

$31 *$

32.

31.

33. 


\section{N D E X}

Coccum Gnidion 34: Crateogonos

$37 \%$

Coccus Baphicus ibidem crethmon

$37 \%$

coccimeles

ibidem.

Crimnon

$37:$

colla

ibidem?

Crocodylion

38.

Colocyntbe

ibidem

Crocus

$38:$

Colocynthis

ibidem.

Cromnion

38.

Comarus

ibidem. Cuminum

38.

Conia

ibıdem: Cucumis

$7 \sigma$,

Conizs

ibidem.

Cucumis egreftis

60.

Corallum

36.

Coris

36 ,

Cucurbita fylueftris $\sigma_{2:}$

Corisherba

36: Cyantos

38.

Corianum uel Corian =

drum

Coronopus

Coftus

Cotyledon

Cochlis.

Cornus

Comon

conos

cor

Coagulum

Coaguium teporis

coagulum equinum

Coagulum phoce

crambs
350

$3^{6 .}$

36.

36 ,

36,

37.

39.

39.

80.

57:

$57^{\circ}$

572

57

37.
Cyclaminos

Cycldminosalters

cypariffus.

cyperus

Cypros

cytifum

D.

38.

38 .

38.

$39 \%$

39.

399

17:

17.

17.

$17^{\circ}$

17.

Daphne

18,

Dictamus

Diontitis

18.

Diphriges 
I N D E X

Dipfacon

Dorycnion

Dracontium

Dryopteris

E

T Benus

Eechinus

Échinusterreftris

Ecbinus marinus

Ecbion

Elaterium

Elative

Elapboboftos

Elenium

Eleofelinon

Elephas

Ellebortus

Elichryfon

Elxine

Elxine

Eltmus

Electrum

Empetron

Enula campana

Epithymum

Epimydion!

Epımelis

Epipattis
18. Ephemerors

ibidem Ephrydros

ibidem Erebinthus

ibidem Erice

Erynos

29. Erythrodanum

23. Eryfimon

23. Eruca

ibidem Euzomon

ibidem Eupatorium

ibidem

$20 \%$

ibidem

ibidem

ibidem

ibidem

21.

ibidicm

ibidem

tbidem

ibidem

24.

21.

20.

21 :

ibidem

ibidem

ibidem
Euphorbium

\section{F}

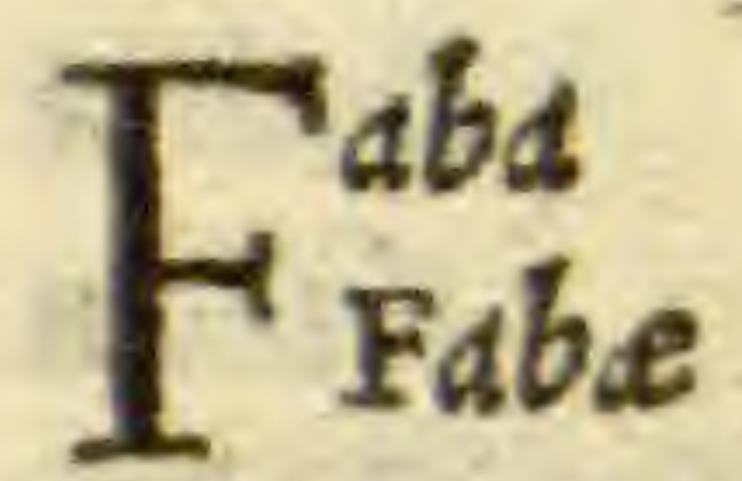

Fermentuns

Ferrum

Fexuini

Fex aceti

Fonum grectum

Ficus arbor

Ficus agreftis

Ficus

Flosfalis

Fuligo pictorum

Fumusterre

ibidem

ibidem

72.

220

ibidem

ibidem

$+98$.

750

23.

60.

66.

67.

75.

64.

65.

76.

5.

10.

29.

41.

74. 
I N D E X I

\section{G}

Calion

$T^{\text {Galipfis }}$

Galiopfis

Garos

Garum

Gilld.

Gallorü faginatorüteftes

Gentiana 15 .

Geranion

Gigarta.

Gingldium

Glaucum

Glaux

Glechon

Glycyrizon

Glycy ides

Gluten

Glandes

Glandule

Graphalium

Gongylis

Gorgylon

Granum tinctorum

Gramen

Gumme ccraforum

Gummi
(80.

Gyris

$$
\text { 14. Gypfos }
$$

ibidem

ibidem

ibidem

ibidem

31.

15.

16.

ibidem

ibidem

ibidem

ibidem

ibidem

ibidem

34:

78 .

80.

27.

$17^{\circ}$

ibidem

$34^{\circ}$

1.

31.

34.

Hepar

Hedera
F arena

6.

$5^{\circ}$

Hediofmon

Hedyfaron

$24^{\circ}$

ibidem

Heliotropium

Hemerocallis

Hemonitis

Hepar capre

Hepar capri

Hepar lacertie

Heparlupi

Hepar afini

Hepar apri

Heparmergi

ibidem

ibidem

tbidem

ibidem

ibidem

ibidem

ibidem

ibident

ibidem

ibidem

ibidem

32.

Hippocampus 260 Heppomarathron ibidem Hippolapathon ibidem Hippopotamus ibidem Hippofelinon

27.

Hippuris

ibidem

ibidem

67.

Hidnars 


\section{N D E X}

Hidnon 67. Lapathon 39 . Hyacinthws ibidem Lapidespreciofioes. 41 .

Hyalos

Hydrargyros

Hydropiper

Hioftiamus

Hypericon

Hypecoon

Hypogloffon

Hypophafton

Hyffopum

I

Tea, radix

Ichtbyocolla

Ios, uiold

Iris

Iris fylueftris

If atis

Ifopyron

Ited

I uncus quadratus

Ixos

\section{L}

T agopus

1 Ladanon

Lathyris

Iapfara ibidem Lapis Iudaicus

ibidem. Lapisafius

ibidem

ibidem Lapidesquiin $\beta$ oğijs. 43

ibidem Lac

ibidem Lactuca

14.

68. Leontopeialon

ibidem Lepidium

ibidem Lepis

ibidem Leucacanibon

Leucas.

26. Leticoion

27. Lence

26. Lemnia terra

27. Lentifcus

ibidem Lenticulapaluftris 68. ibidem Lentes

ibidem Limomium

ibidem Lichen

29. Lichenes

26. Libanotides

Libanotus

39. Lignys

ibidem . Ligufticum

ibidem Litargyros

ibidem Luthofsermon ibidem

40.

ibidem

ibidem

ibidem

ibidem

ibidem

ibidem

ibidem

$41 \cdot$

15.

65.

$4 x^{\circ}$

ibidem

ibidem

ibidem

ibidem

Lino
- $43^{\circ}$ 
Linofpermons

Linozoftus

Litrum

\section{Lilium}

Lonchitidis

\section{Lotos}

Lotos agreftis

Lotus arbor

Lotos Aegyptid

Lolium

Iutum Aegyptium

Iycopfos

Iycium

Lyfimachium

Iychnis

M

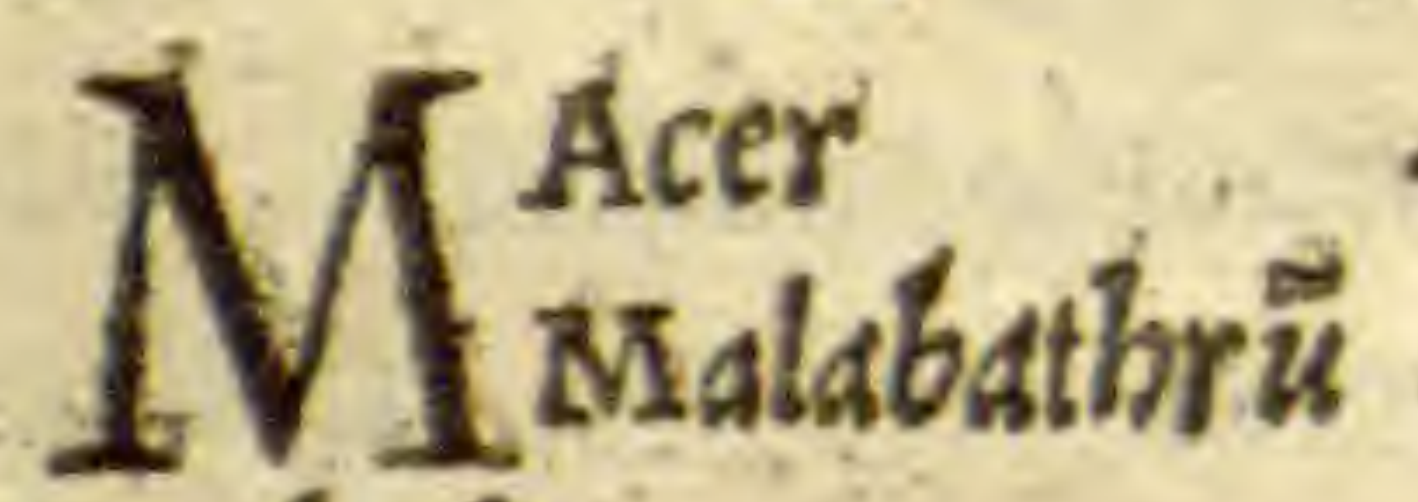

Malache

Mamiras

Mandragora

Mannd

Marathrum

Maftiche Cbia

Maftiche Aegyplis

indius perfica

Mals
43. MalusPerfica $47 \%$

43. Mala Armenica $\quad 47^{\circ}$

45. Marrubiumnigrum 22 :

37. Marrubium 56.

43. Maldpunicd 27

$44^{\circ} \mathrm{Mazd} \quad 75^{\circ}$

44. Melanterid $45^{\circ}$

44. Melanthum $45^{\circ}$

44. $\mathrm{Mel} \quad 454$

3. Meldmarumi $\quad 45^{4}$

is Melia 46

$44^{\circ}$ Melitotumi $45^{\circ}$

$44^{\circ}$ Miliffophyllon $\quad 46^{\circ}$

44. Memecylos 46.

44. Mefpild 46:77:

Medion $\because 47$.

44. Meon 47.

44. Medulla 48,2

44. Mentsodorata: 23.

44. Mercurialis : $43^{2}$

45. Melopepon 76

45. Milax 47

45. Mify $47^{i}$

45. Myagros 47 ,

45. Myaces $47^{\circ}$

46. Mituli 470

46. Myaron $48 \%$

Myagros 
I. N D E X

Myagros, herba Myélos

Mitetes

Myos witis

Myza

Myrice

Myropbitlon

Myrrha

Myrrhis

Myrrbone

Myrtus

Millum folis

Mirabolanum

Milumm panicum

Molybdena

Molybdos

Moly

Molles pifces

Mufteld

siullus

\section{$\mathrm{N}$}

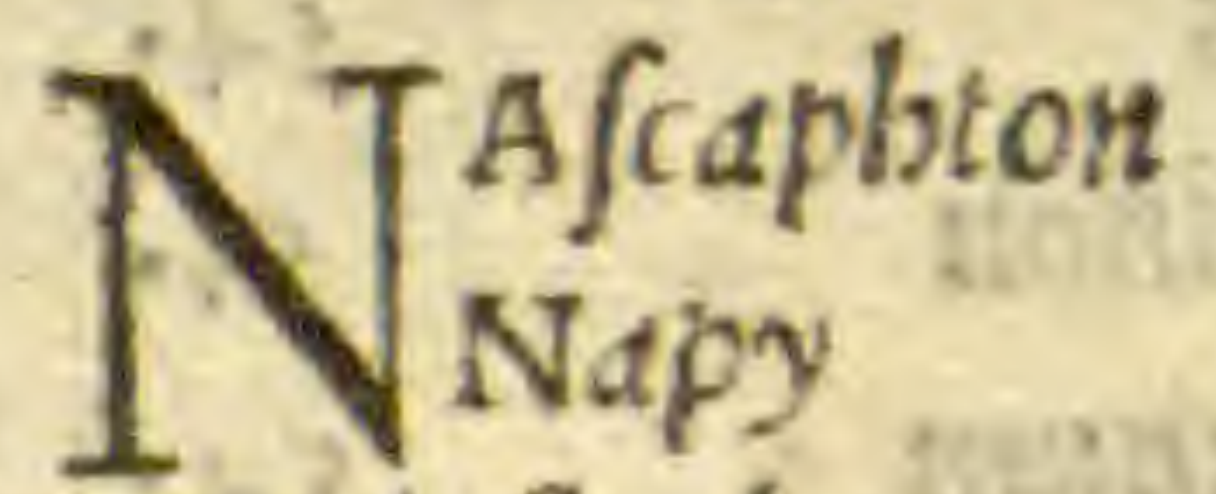

Nardoftachys

Nardus Celtica ibidem

Nardus montand ibidem Nartex
48. Narce

ibidem Narciffus

tbidcm Nafturcum

ibidem Neuras

ibidem Nerium

ibidem. Nimphed

ibidem Nimphedalba

ibidem Nuces

ibidem Nux Pontica

ibidem

ibidem

43.

12.

75.

47.

ibidem

45.

83 .

14.

82.

48.

ibidem.

49.

ibidem
O

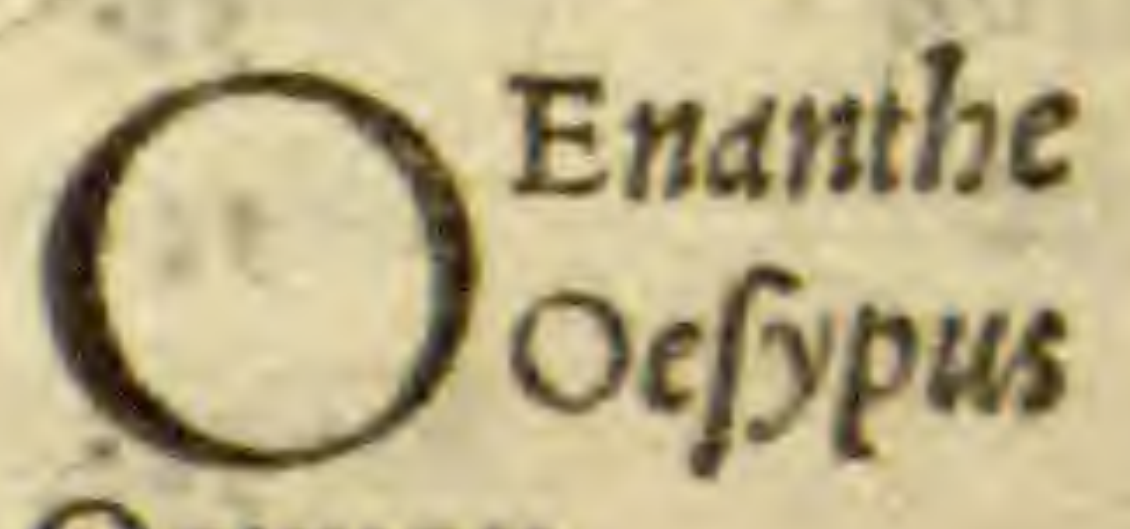

Ocunon

Ocimoides

Olinthicraßi

Oloftum

Olea
$\mathrm{N} u x$ ducllara

Nuxpini

$\mathbf{N} u x$ unglans

ibidom 49.

$29 \cdot 72$.

$49^{\circ}$

ibtdem

ibidem

ibtdem

29. 30.

ibidem

$77^{\circ}$

78 . ibident

so.

ibidcms

19.

Olee Acthiopice lachrim Oleumuetus 19. (ma.19) Oleumexdrupis ibidem Oleŭ ex crudo omphacio Oleum ablutü 19. (19. Oleumagreftisolee 20.

Olcuint 
I N D E X.
Oleum Cicinum
20.
Othonnia
50.
OleumSefaminum 20. Oua
Oleum Raph
Olcomel
Omphaciam
Oullacaro
79.
Ondzra
Onoma
Onofina
Onobrychis
20.
20.
Oxyacanthos
79.
50.
50.
50.
1 Apaker
P
OniAfini
Onyches
Ononis
Origanus
Oreofelimons
50. Pancration
50. Paliurus
51. Pandcois
51.
51. Papyrus
46.
10.
$53^{\circ}$
53.
$53^{\circ}$
53.
51. Paronychid
53.
51. Paffer
670
5. Paftinaca
Orobanche
Orobos
Orminon
Oriza
Orchis
Orchis minor
51. Pentaphyllos
$74^{\circ}$
51. Peplos
5l. Peplion
51. Pepon
51. Periclimenon
53.
53.
53.
$53{ }^{\circ}$
$54^{\circ}$
52. Periftercon
$54^{\circ}$
Orchis Serapia
52. Petafitis
Oricha
Ofiris
OfJabumans
75. Petrofelinon
52. Peucodanum
$54^{\circ}$
54.
$54^{\circ}$
52. Peganon
52. Pellis ouind
$54^{\circ}$
O) $\int a$ funm
52. Pellishippotdm
8.
Ofraca
oftrea
52. Pbaci
28.
68.
Phacos 


\section{N D E X.}

Phacos

68. Pifcium iura

Phalaris

68. Platanus

Phatangites :2

68.

Plumbum iuftum

68. Polemonitum

Philyras

6. Potium

Phlomi

Phoenix

69. Polygalon

69. Polygonon

Phoenux arbor

Phormion

Pbu

Phycos

Pbyilitus

Peper

Piper album

Piced

Piffa

Pi $\int$ feleon

Piftatia

Pitydes

Pttyocampe

Picee cortcx

Pityufa

Pinus

Picrocomon

Pytia

Purum agrefte

Puli

69. Polygomaton

69. Polycnemon

क. Polypodion

69. Pompholix

69. Potamogiton

53 Poterrhion

53. Portulaca

54. Porrus

ibidem Praßstum

tbidem Prafon

ibidem Propolis

ibidem Prunus

55. P eudobunion

ibidem Pfcudodictamum

ibidsm pfillion

56. Pforicon

57. Piamice

57. Pteled

it. Pteris

6\%. Pulnoagninus
55.

$47^{\circ}$

$55^{\circ}$

ibidem

ibtdem

ibidem

ibidem

56.

ibidem

ibidem

tbtdem

ibidem

7.

$44^{\circ}$

56.

ibidem

ibidem

34.

70

ibidem

ibidem

ibidem

$56^{\circ}$

ibidem

$57^{\circ}$

$55^{\circ}$ 


\section{N D E X}

Pulmo fuillus

Pulmo unlpis

Pulmoma

Pulegium

Pulicaria

Pumex

Pyrethrun

Pyros.

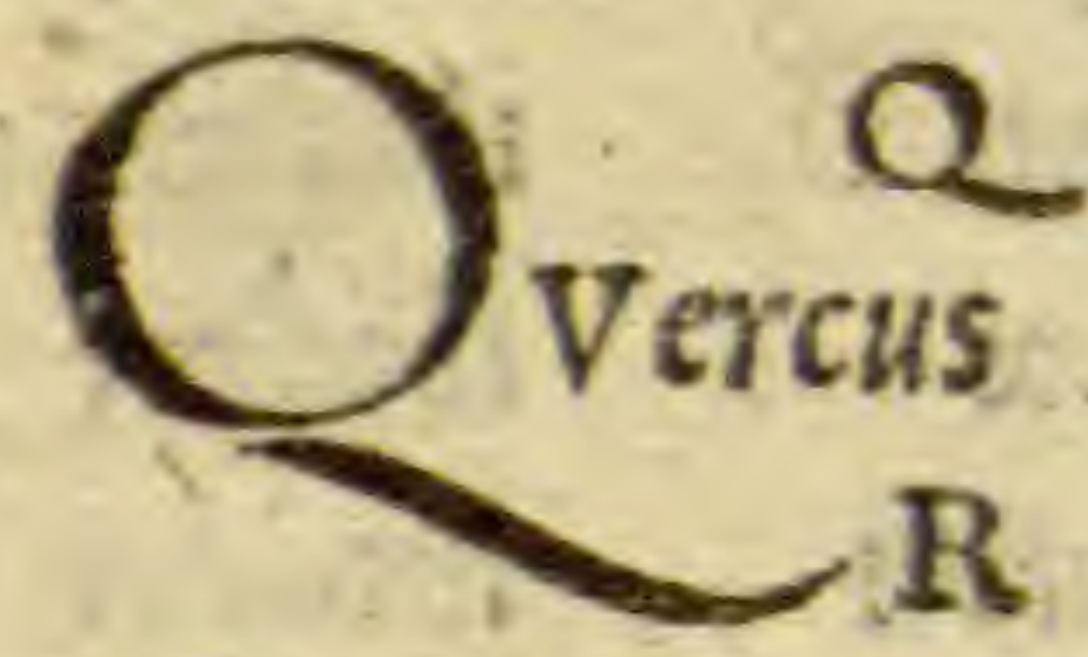

DAcos

R Rhammos ibidem Raphanos

Ra

Radix Rhodia

Radicula

Reon

Rhefine

Rhefinapica

Rhefind frobilina

Rhefind abietind ibidem. Rbefind terebintbund. 58 . Rhypos

Rofa

Rhus

\section{7}

ibidem

ibidem

58.

ibidem

73 .

58.

ibidem ibidem

58. ibidem ibidem tbidem:
55. Rubus ibidem Ruta tbident

56:

16.

$34^{\circ}$

32.
- 12.

$54^{\circ}$ $S$

59.

ibidem ibidem ibidem ibtdem ibidem ibidem ibidem ibidem ibidem $i b i d e m$ ibidem 60.

Sanguis fuillus 3. Sanguis columbarum 3 . Sanguisnocture ibidem Sanguis Veßpertilionü 3 : Sanguts caprarü ibidem Sanguisgalli ibıdem Sanguisgallne ibidem Sanguisouillus ibidem Sanguis hoodorü ibidem Sanguis urforum ibidem Sanguiscaprarü ibidem Sanguis 


\section{N D E X}

Sanguisterreftriscroco $=$ Seffeli $\quad 59 \div 1 \%$ ?

\section{dyli}

Sabucus

Sales

salfugo

Sabina

Sachar

Sarguis

Scammoisid

Scandix

Scincus

Scilla

Scolopendra

Scolopendrion

Scolimon

Scordions

Scorodon

Scorodoprafon

Scorpioides

Scorpius

Scorla

Scoria plumbi

schinos

schinu Anthos.

Schunifios

Schiftos

Sclinon ibidem sepie Tefta

4. Sefamoides

4. Sefamum

5. Seps

13. Senceta anguis

45. Senecta $A$ /pidis

80. Sialon

6 . Sideritis Heraclia 60.

ibidem sideros

ibidem sycios

ibidem sillybon

ibidem silurus

ibidem stlphium

ibidem sifon

ibidem Sifareus

62 Sifymbrium

ibidem syca

ibidem Sice

ibidem

ibidem

ibidcm

65.

ibidem

bidem

42.

59
Sycomorus

Symphyton

symphyton minus

siligineus panis

simulaceus paris ibidem

silique

syrtact

76.

$64^{\circ}$

ibidem

65.

ibidem

$65^{\circ}$

$74^{\circ}$ ibidem

ibldem

ibidem

ibidem

ibidem

ibidens

ibidem

61.

ibident

ibidenz

.




\section{N D E X}

Sycomora $\quad 78$ Stoebe

Sylueftridanimalia so. Stoechas

64.

Smilax

62. Stratiotes

Smyrna,Beotid ibidem Strychnon

ibidem

Snyrnium

ibidem

Stypteria

Sonchus

ibidem. Styrax

ibidems

ibidem

ibidem

ibldem

Soldnus

64. Stercus

35.

Solarytum fomniferu $64^{\circ}$ stercus caninum ibidcm Sclanium furiofum. $64^{\circ}$. Stercus pueror $\ddot{u}$ ibidem Sordes balnearum

17.

Stercusluporum tbidsm

Sordes

58. Stercus caprarum ibidem

Sordes durium

Spsrgantion

Spartum

Spangos

Spdingid

Spodion

Spind Arabicd

Spind Aegyptid

Spina falis

Spinalis medulld

sphondylion

squama aris

Sqquama ferri

Staphilinos

Stachis

Sirdta

Stimini ibidem

62 .

ibidem

63 .

ibidem

ibidem

4.

ibidem

S.

80.

65.

40.

ibidem

63.

ibidem

ibidem

ibidem

Stercus boum

ibidem

Stercus ouium

tbidem

Stercus columbarum 35.

Stercus domeftes arü auıu

Stercus muriu $35^{\circ} \quad$ (35. Stercusciconiarü ibidem stercusfturni ibidem Stercus crocodylorüter =

reftrium

Sulphur

Sxdor

Sulle carnes

$T$

Talum bubalum

Terra

52

5

Terraculta

15

15 
I N D E X X

Terra Acsyptis Terra finopid

TerraMiltos

Terraselufina

Terrasamid

Terra ampelitis

TerraCretica

Terra Eretrias

Terra Pegntis

Terra Armenid

Tefte

Tefta Scpie

Thalietrum

Thapfia

Thelipteris

Thlapis

Thridax

Thymus

Thymbra

Thymeled

Titanos

Torpedo

Tragacantha

Tragiume

Tragium frutex

Tragoriganos

Triobolos
15 Tragositodes

15 Trigle,pifis

15 Tripolum

is Triches

15 Trigon Thalaßia ibidem

15 Troglodytes.

15 Trifolium

15. Triticam

15 Thberum fuccus

67. 46 . $7 \cdot$ 74 .

\section{V}

52

59

25

25

25

25

25 Vitisalba

26 Vitss fatiud

26 Vitisculia

26 Vitisnigra

65 Vitisagrcfitis

49 Vipera

66. Vifcum

ibidcm Viola

ibidem

ibidem

ibidem
Vinum

Vitrum.

Vini potentid ibidem ibidcm ibidem
66.

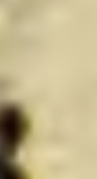

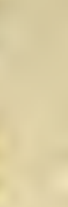




\section{N D E X}

Vintum dulce 8, Vterus

Vinumacerbum ibidem Vulpes

Vinumfuluum ibidem Vuapaffa

Vinumuetus ibidem. Vulpis pulmo

Vlmeus

56.

Vuspafje

$8 x^{\circ}$

Vmbilicus Veneris 36. Vue

Vrtica

3. 72 .

Vrina bumand

Vrins muli

52.

Vrindcaprarum ibldem

Vrina pueri impubis : $52^{\circ}$

53. 2 Xiris ibidem

55. 76. 76.

Vruns camelorum. ibidem

(2) $F \perp N \perp S$ :

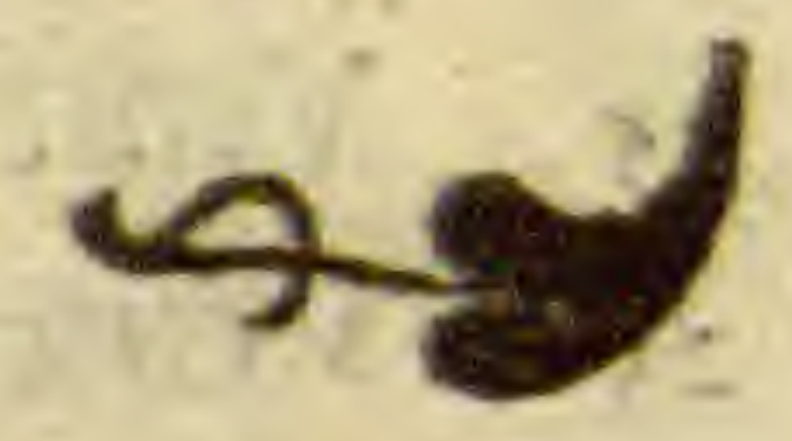

$\mathrm{x}$

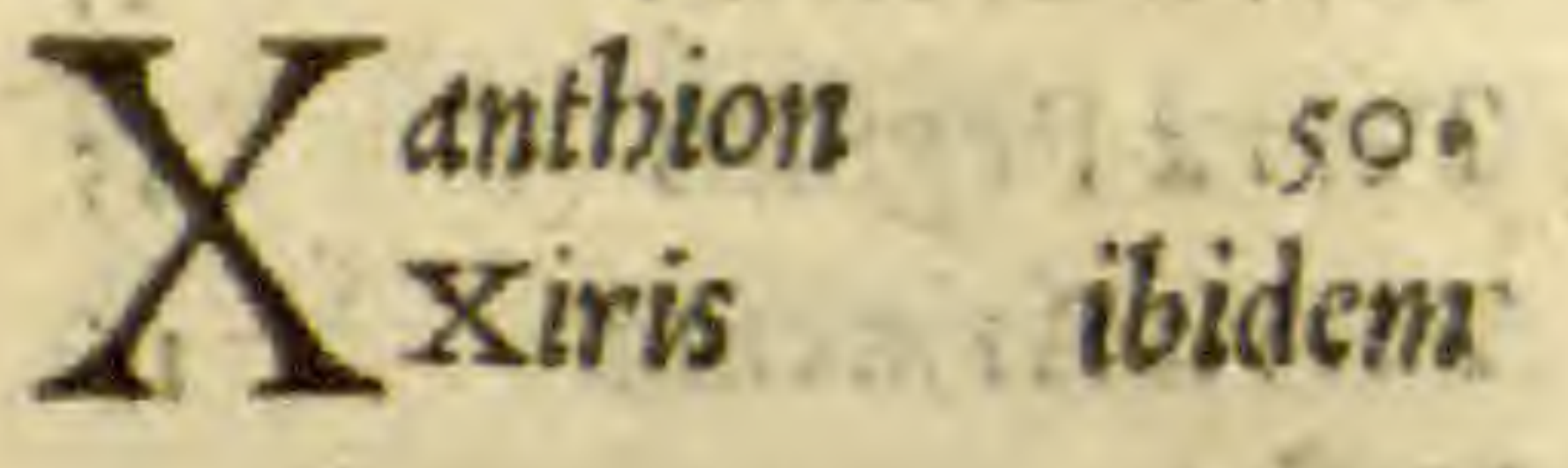
PAVII AE G I E T AE
V I T A EX
$S \vee$ I D A.

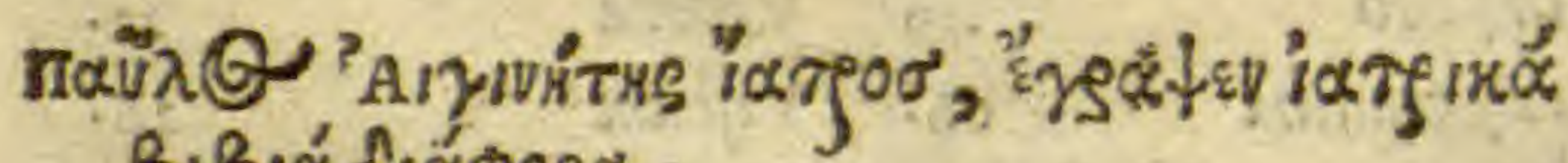

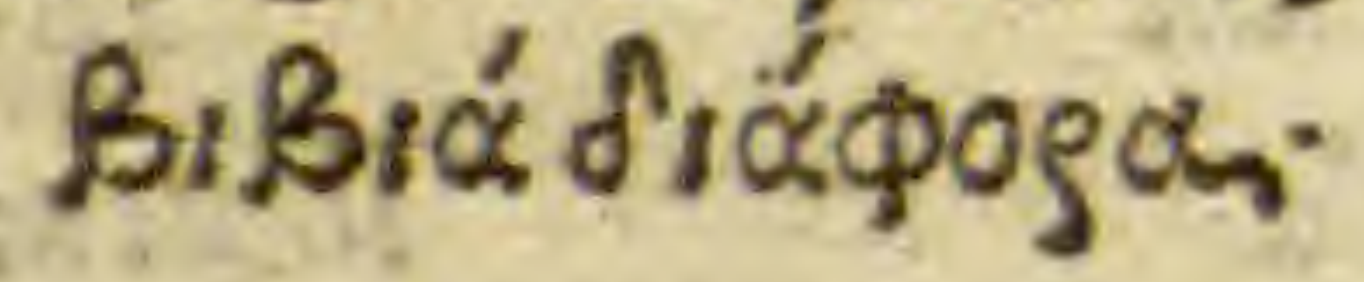

PAVLVS AEGINETA MEDICVS, \&fcripfit libros medicinales diuerfos. 


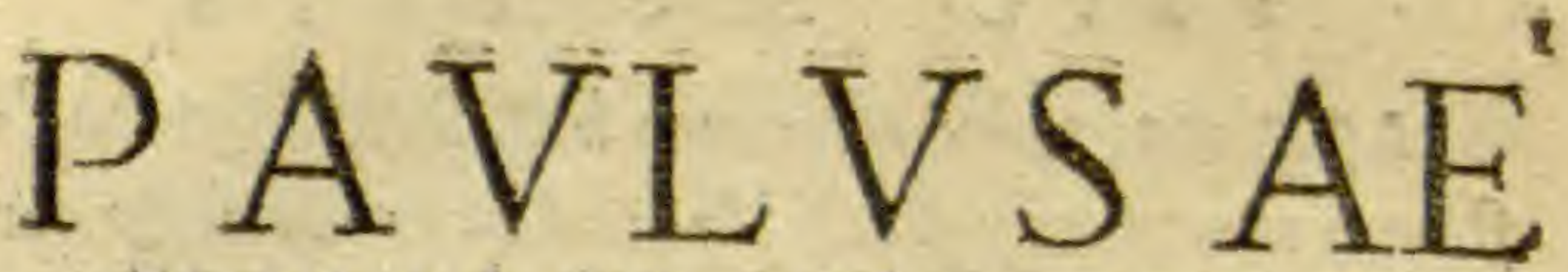

GINETA DE SIMPLICIBVS. Othone Brunfilfio interprete.

\section{Abrotonum.}

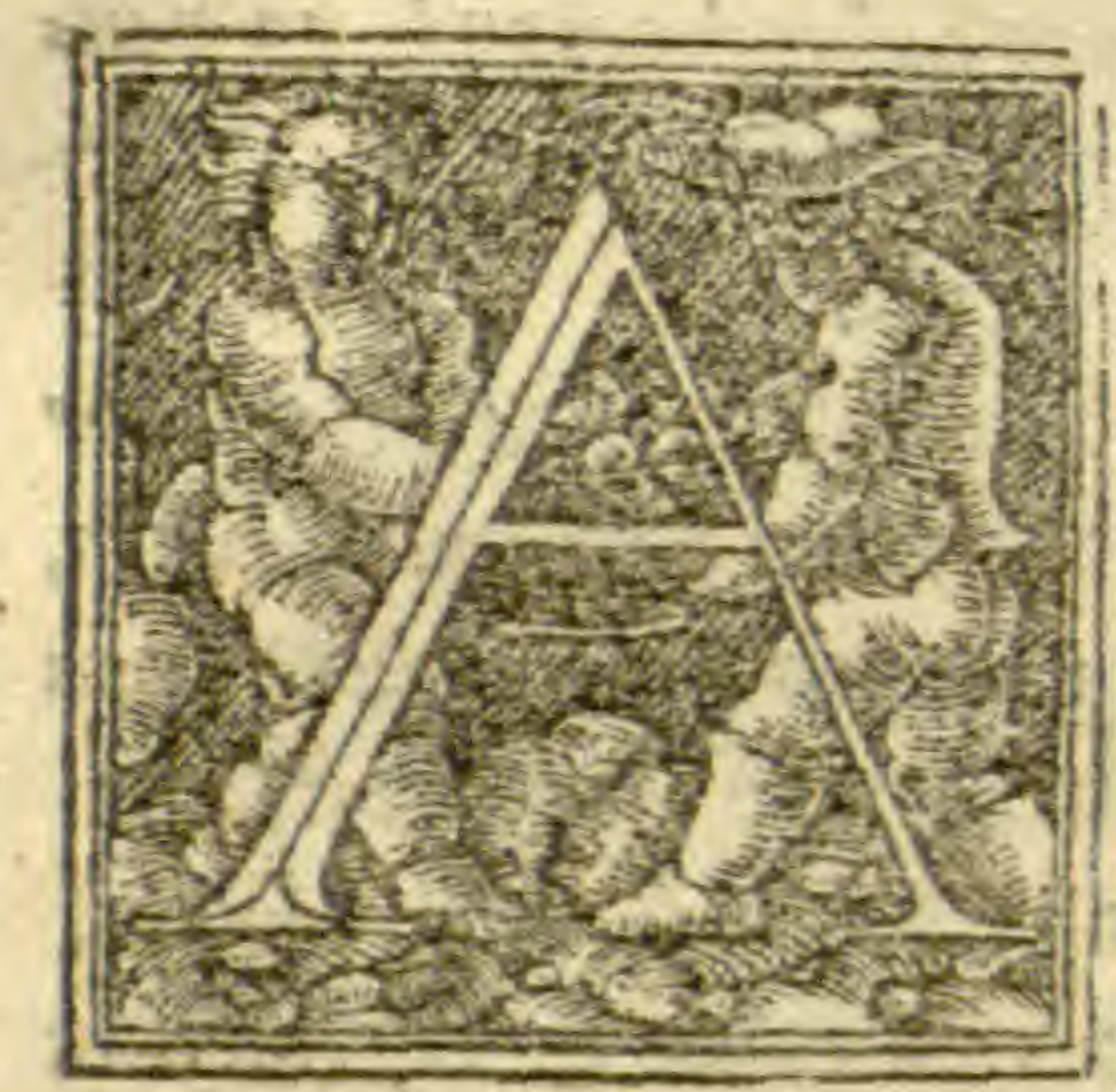

Brotonon, fimul or cale facite ficcat, id'́; adeo in tertio ordine. Nec mi= nus digerentis, er th ter tio ordine incidendipoten tie. Vnde per totum cor = pus intritum, que per ac ceßsionem redeunt fubirs a de rigores, anat $\cdot$ Cate $=$ rumuentriculo inimicum. Vftum quog; magis magisớs ficcat. Alopecias fanat cum olco quopiam quod parti : um tersuium fuerit.

\section{Agallochum.}

Agallochum, lignum eftex India, Thyis fimile, o= doratum, $v$ quod commanducatum, oris halitum com mendet. Ad uapores fuffitus q; accenditur - Radix eius pota, drachme pondere, uentriculiaquo fis humoribus fimul at $q_{;}$debtlitati atuxiltatur. Preterea $\sigma$ iectnoris - inteffinorum torminibus accommoda, or pleuri tidi, id eff, lateralibus doloribus.

\section{Agaricum.}




\section{P A V I. AE G I N E T A}

Agaricon, radix eff trunco harefcens, fed con/is Rentia admodum molleufcula, coalita exaêred $\sigma$ ter

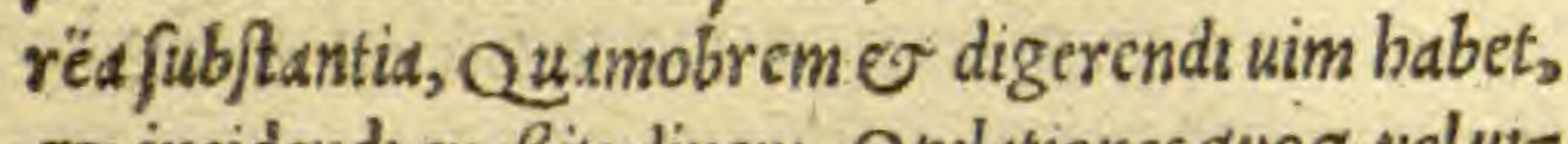
- incidendi craßitudinem, Optlationes quoq; uel ut= fcerum prefacturas repurgandi.

\section{Ageranton.}

Agcranton, facultatem habet digerendi, or paus . latira quodammodopblcgmate liberands.

\section{Agnus caftus.}

Agnusuellygos, $\Theta$ calefacit $\odot$ ficcat in tertio or dine. Eft autem partum tetuuium, of flatus difctutiěs, Ad conferuandam caftitatem prodeffe credutur', non folumcommeftus uel potus, uerum etiam fubftratus. Scminis quoq; eius ufus eft, adinduratum lienem, Obepar, fi potetur. Frixum tamen o inflat minus o digeritur facilius.

\section{Agroftis, id eft, grametr.}

Agroftis, tum ed imprimis que cft ex Parnafo mons $t c$, preciofacxifftr. Siccal enim mediocriter, o refri = gerat nonadco, parisum tenuitatcm obtmens, $\odot$ fub= acerba, quapropter mulinera glutinat, cataplafmata, $\sigma$ lapidofa ue fice excrementa frangit-

\section{Anchufe quatuor.}

Anchufe, quaruor generd, non unius quidemo= mnes potentie fint. Nam que ovor $\lambda \lambda^{\prime} \mid \alpha s$, id eff, ono cleadictaeft, eius radix con/tringendtumres babet, $\mathrm{F}$

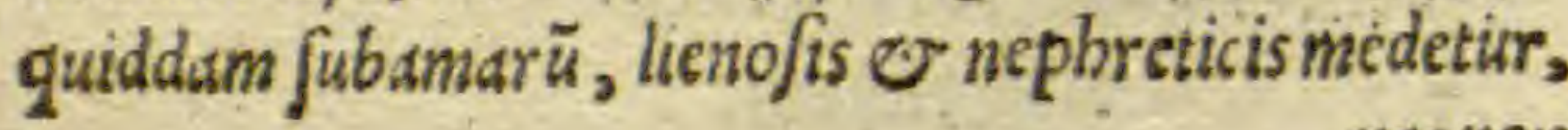




\section{D.E SIM P LICIBVS.}

nec non $\mathcal{O}$ Erefipilatis, nimirum cataplafimata. Et licet folla ip fa radice minus refrigerent, at q; exiccent, tamen ad d:arrboeam precipue bibuntur. Caterum

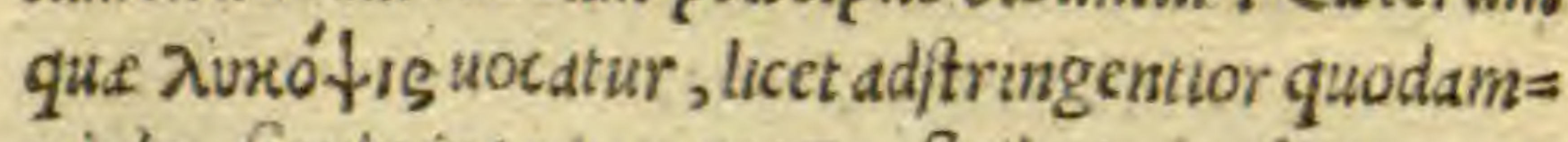
modo, fimiluer tamen er Erefipilatis cuxtlutur. At Onocles, of Alcibiadie cognominate, uis mator me dicata, $\mathcal{O}$ cos dịu ḋuperis dcmorfi funt, admodum

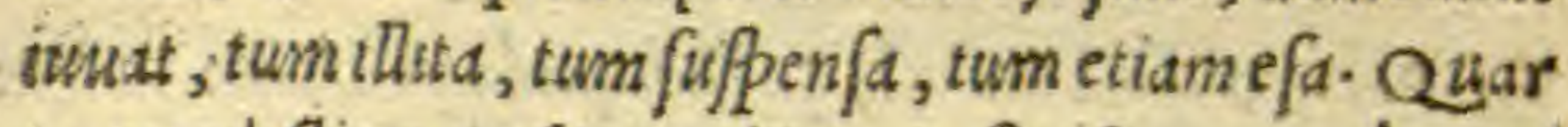

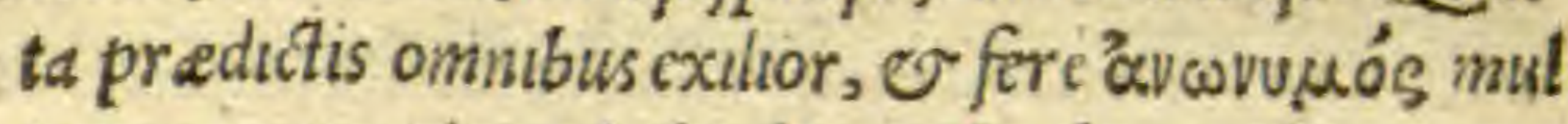
to tamen amarior Alcibiadio oxybaph imenjura pota, latos lumbricos expellit

\section{Adarce.}

A darce, effentia quidem fus, uelut fpuma quedam eft, aqua falfe concreta, circum harundines concre $=$ fcens, acerrima eo maiorem immodum calda, adeo ut adurat. Nullus eiuis ufus eft, $m / f$ foris appofita, $m i$ ftaq́; aligs. Namintro in corpufculum ipjam fumere, haud quaquam fas est.

\section{Adianton, uel capillus Veneris.}

Adıanton, arefact, extenuat $\mathcal{O}$ diftrahts, $\sigma$ in calore quidam or frigore, fynmetrum eft, quecau $\beta=$ fa eft, quod capillorum defluuia explet, strumas q́; et abfceffus digerit, ium preterea uefice calculos commi nutt, epotum, $\mathcal{O}$ craßis é pulmone excreatiombus confert, adeo óp deftillationes in ftomachum fijtic:

\section{Aizoon.}

Aizoum, geminume ef, o utrumóg.refrigcrat quie 


\section{P. A V L. AE GINET A}

dem in tertio ordine, fed arefacit, $\mathcal{E}$ ddffringit medis ocriter-Itaq́; aduerfumignem facrum, o berpetas et phlegmonas d fluxione natas, accommodatur.

\section{Aetonychon.} bebitur.

Aetonychon, de quo in lapidibus fermo nobis has

\section{Athera tuel Äthara.}

Atherd, forbitio eft, quafi liquida pulticuld, ex zed uel tritico, in tenui ßimum pollinem molita, infantibus accommodata, e cataplafmatise effc $d x$.

\section{Aegylops quæ \& Aueria.}

Argylopsdiftrahendihabet uim, quod ex coliquet, quia phlegmonas induraicas, e agylopas fanat.

\section{Aegyros.}

Acgyros excalefacit in primo temperatorum ordis ne, ficcat uero medlocriter, eff'; partum ternium. Huxius folla cum aceto catapla fmata, podagricos dolo res fedät. Verü, que ex ea fillat, rbefina, floribus paus. lo calditior, mifcetur acopis, nec non e malagmatis Fructus item cum aceto potus, Epilcpticosrefttuit.

\section{Pudendum mafculi cerui.}

Genitale mafculicerui, ficcatum, trttumq́; cumuis no, e potum, d̀ uiperd mor fis auxiliatur, mifcetur $\checkmark$ compofitis medicamentis.

\section{Aethale.}

Aethalc $\odot$ lignys, lttera L - differentur .

\section{Aethiopis.}

Acihiopis, fimiliaphlomo foliahabet, cius radicis des coctum 


\section{DE S I P L I C IBVS.}

cocium, di q́; fubinde potum, ifthiadicis, pleuriticis, O b b mopiotcis remedio eft. Eademarterias $\mathcal{O}$ afperi tates cuni melle fanat.

\section{Sanguis faillus.}

Frigidus fanguis, nullius frugi bone eft. Suillus bu midior, fed minus calet, o bumano fanguini, tempe $=$ rie ipfarefpondens.

\section{Sanguis columbarum.}

Columbarum, $\mathcal{O}$ palumbi, $\mathcal{O}$ turturis janguis quodammodo temperatus cxiftit. Que in oculis funt vтоб $\phi \alpha \gamma^{\prime} \mu \alpha \tau \alpha$ fanat, fi calldus inftilletur, $\sigma$ me ningi infuJus, qubuscapita fucrint per forata, inflam = mationem arcet.

\section{Sanguis noctur.}

Noctuse fanguis, cum uino potus, uel aquat, difproes medctur.

\section{Sanguis Vefpertilionum.}

Sanguis Vefpertllonum, fi uirgunum uberibus il= liniatur, ab extuberattone tuetur. Ferunt $\mathcal{O}$ pilos pro bibere, ne fubinde repullulent, fieoperung atur locus, perinde ut fanguinem quo ranarum, ev chameleontis, o mufce canine, uel cynordiftarum. Quanqu Galcnus id non ita babere affirmet, ficto reipericulo.

\section{Sanguis caprarum.}

Sanguis caprarum, propter tmmodicdm ficcitatem ficum melle potetur, fubeunibus cutem aquib, auxi= lioeft, o in rembuscalculos atterit.

$B_{3} \quad$ Sding $=$ 


\section{P A $V I$. AE GINETA}

\section{Sanguis galli $\&$ gallinx.}

At domefticarum aulum, id eft , galli 0 galline fanguis, è meninge fluxum fanguinis retinct.

\section{Sanguis ouiltus.}

Ouillus eplepticos 0 hamorragiam precipue iuuat.

\section{Sanguishodorum.}

Hodorumetiam fanguis recens datus, menfurd fea micotyle, cum equia portione acetipotus, hemoptos icis, ideft, ex thoracefanguinem fpuentibus, utilis cst. Sanguis urforam \& caprarum.

Vrforum, er agreftium caprarum, of bircorums $\checkmark$ taurorum fangunem abfceffus concoquere ferunt. Sanguis terreftris crocodili .

Terreftris crocodilifanguis, uffum acuit, admift fariorum q́; equorum fanguis, fepticis mifcetur.

\section{AeraLolium.}

Aerd, calcfacit $\mathcal{O}$ exiccat feréut tertio ordine, $v$ iridi aqualibus utribus pollet.

\section{DeAcallide.}

Acalis, frutcis culusdam fiuctus est in Aogypto nafcentis, cuus dilutumi cqIyrijs ad claritatem uifus uti libus mifcetur.

\section{Acacia.}

Acacia, tertij quidemeft arefacientium ordinis, fed refrigerantium fecundt, fiquidem lota fuertt, cft cnim peraccrba e terrofa.

\section{Acalyphe, ideft, Vrrica.}




\section{D.ES I.MPIICIB VS:}

Acalyphes, buius herbe tum fructustam folis te= nuium partium funt, ficcant $q$; fine mordecatione. Tus bercula dif cutit, of uentrem fubduct, inflandi quớs; uires babet, fed mediocriter, propter quod vencrem cximulat.

\section{Achantos, fpina unigaris.}

Achantos, alij equidem Melsmphyllon appellant, alij Paderotam, diftrahendie exiccandiurres obturet. Achantion.

- Achantion, partium tcnüu or caldde potentie eft, conuulfis modetur, $d c \beta p a f m i s$.

\section{Acanthaleuce, fpina alba.}

Acanthaleuce, radicem bibct exiccantem, $0 \mathrm{~d} d=$ fringentemtemperatc. Quäobrem o Coliacos, Sto machicos, hemoptoicos, $\mathcal{O}$ dentum dolorelaboran $=$ tes iusat. Porro, femen attenuantium purtium, o cali de potestatis cft, quod conuulfionimedetur:

\section{Acantha Aegyptica, id eft, Sp̈ina Aegy}

\section{pria uel Arabica.}

Acdntha Acgyptica, alij uocant Arabicam fpindm, admodum ad/fringentis $\mathcal{O}$ deficcantis potcntie $\mathrm{eft}$. Quofit, ut non tam fanguinem, quàm dlios quógf fuo resretincat.

\section{Aciros.}

Aciros, ocimo perfinilis, ftringit medlocriter, ideo q́; tum uentrem, tum menfes fiftit, potd, cataplajs matdetium, phygetla, of Erefipilatd unat.

B 4 AcOH 


\section{PAVL. AE GINETA}

\section{Aconitum.}

Aconitum Aconitum, quod o pardalianches, feptice v des lycoctonö. leteria facultatis, ad intra, id eft, incibo potuóf fum= Germant= ptum. Ceterum foris adputrefaciendum o exeden = ce vuolff = dum carnes, $\sigma$ que è corporc erumpunt, idoneum.

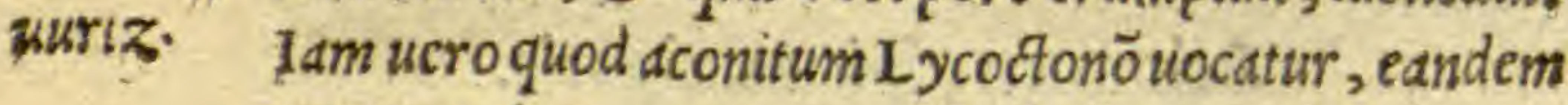
propemodum cum priore uim habet.Peculiare, ut ur fos er lupos perimat, quemadmodum illudpardos.

\section{Acorus.}

Acorum, ordinus eft tertij, calefaciendifalicet or deficcandi. Vtumur autem radicc etus ad ciendum uri nam, ov ad lienem induratü, Ceratoidis infuper craf = fitticm detergit, fimul at $\dot{q}$; extenuat .

\section{Locuifx:}

Acrides, idcft, locufte, fufftite, urine anguftijs,

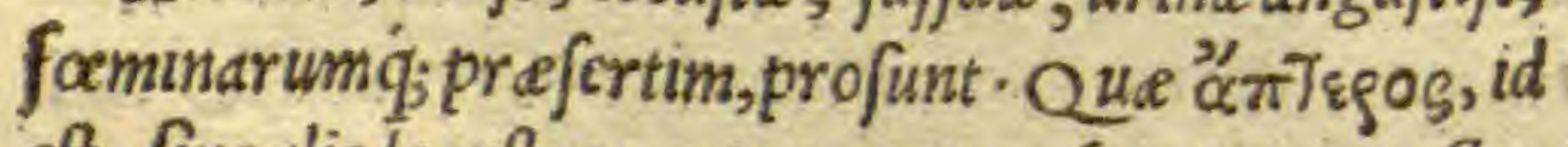
cft, fine alis locufta uocatur, contra fcorpsonumictus sumuino utilipime bibitur.

\section{Sabucus \& Ebulas.}

Acte qua miagna, er chameacte, ambe arefacien di uim habent, $\sigma$ mediocriter digerendi, $\odot$ tam po= te, quadme etiam commefte, bydropicorumaquam fub. ducunt.

\section{Sales.}

Omnes fales ficce uebementer, $\sigma$ ddftringentis funt potentia, o quicquid in corporibus humidume ft depafcunt, [uaq́q, mordacttate fubducunt. Qu uare ad fal 


\section{DE SIMPLICIBVS.}

famenta eis utuntur, $\mathcal{O}$ d̀ putredine feruant, uffi autẽ magis exedunt.

\section{Althea.}

Althea fiue Eblftus, fpecies eft fylueftris malue, uim babet diffrahendi, furmmittendi, inflammationem arcende, mitigandi, $\mathcal{\sigma}$ tubercula difcutiendi, preter cetera radix, \& femen, uitiligincs abftergit, \& femen calculos atterit-

\section{Alimum.}

Alimum, partium difsimilarium exiftis. Guftufal= fum, $\mathcal{O}$ fubaftringens, maior autem pars eius calis da eft temperate, cum humiditate inconfecta - Semen humanum, ov lac gignit.

\section{Alcaz.}

Alced fylueftris malue, $\sigma$ hec genus eft, cum ui= no potd dyffenterids or mor fus fanat, tum in primis radix.

\section{Alcyonia.}

Alcyonid, omnia detergunt, de digerunt, dcrem qu4 litatembabentia, e tale quidë exiffit Milefium. Pre ftat autě quod eft uermiforme $\sigma$ purpureum. Ita uftü

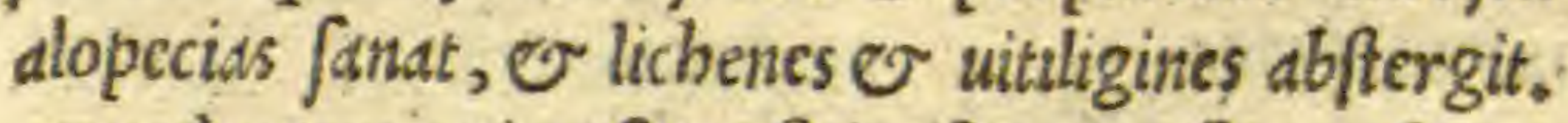
Quod autem extima fuperficie a fperum eff, non folum abftergit, uerum etiam excoriat $\odot$ fauciat - E/t quod lanis fuccedis quog; afsimilatur, fed cotcris ommibus imbecillius.

\section{Halmx, Salfugó,}

B s Salfulu 


\section{PAVL. AEG INETA}

$\therefore$ Salfugo quidem immixta, lcet altcrius fubftantie, confimilem falipotentiam babet, quaqu umbccillorem; aqua ddimixta. Pifcium dutem falforum, ov oluarum, qua Colymbades ditfe funt, uirum magis extergen= tiü eft. Proinde isthiadicis $v$ diffentericis infunditur.

\section{Aloè.}

Aloè, calcfacit in primo ordine, ficcat ucro in ters tio. Eft autem uilde grata ftomacho, ut fi quid aliud, quippe que und uicc abftergit, áigerit, of fercora ex= cernit, quamq gelota minus abftergit. Sedffomacho ma gis eft amica, inflammationes fedat, ov hulcera quie agreadcicatrič perducutur fanat, maxime que curcd fedem funt $\mathcal{O}$ pudenda.

\section{Flos falis.}

Flos falis, medicamenliquidü, magis tenuium pars tium, qudim fales ufti, acuta fatis diq́; diffrahcridi fa = cultatc preditum.

\section{Spuma falis.}

Halos acbne, id cft, fpuma falis, fpumofa eff falis efflorentia, mulio tenuiơris nature, quàm fal ip $\int c, \mathbb{C}$ distrahens etlam multo magis, fcd ad contrabendum minusidoned.

\section{Alfine, auricula muris.}

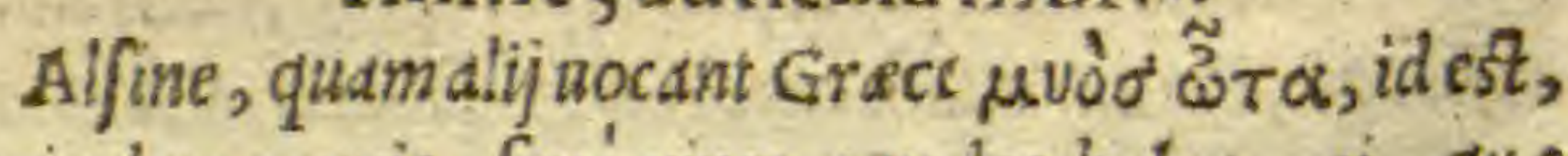
duriculam muris, feré uires equales babet, ei, que dicta eft Hellxire, reftringendif folicet $\odot$. humecidandi. Quacirca for untes fritat phlegmenas. 


\section{DESIMPLICIAVS: 6 \\ Alypon. \\ Alypon, purgat nigrambilem, femen per inferna,}

pari cpithimo menfura fumptum, addtis acto o fale. Intcftinatdmen leuiter cxulcerat.

\section{Alyfion.}

Aly fJon, fic nuncupata herba eft, quia dे cane ra biofo mor fis dtixiliatur. 'E/t autem poteftat's ficcato= rie $\mathcal{O}$ diffrahendl, abftergentis $q_{;}$- Qua ratio= ne renum meat us apertt, $\mathcal{O}$ apperitudinem duriciem '; prank coloris excutit.

\section{Vulpes.}

Vulpes uiuus, Totus uiucns uel mortuus in oleo decoctus, digerendi facultatem indit oleo, ut in pros fundo fixos humorcs in extimam cutem extrahat. Quarc artbriticos in ip $\int 0$, in alueolum imponentes, ac multo ibi tempore immorariprectipientes, princls pium pafionis fedauerunt, diutus autem inmorati eo medioliberatifunt.

\section{Amaracus.}

A maracum, calefact in tertio gradu, ut wocant, exiccat uero in fecundo.

\section{Amarantus.}

Amardntum, ditenuat, or incidit, Menfes, igitur ciet, cum uino potum, o grumos fan guinis diffolut, fi cum mulfo bibatur. Breuter, fluxiones omnes compe

7. foit acdeficcat, fed ftomacho plane perniciofum.

\section{Ambrofia.}




\section{P A V L. AE G I N E T A}

Ambrofia, appofits emplaftrum, uimbabet adflitis gendi, o expellendi.

\section{Ami.}

Ami, è tertiaclaffe eft calefacientium, ov drefacien tium, partium tenuium, quod o digerat, $\mathcal{F}$ urinam mouedt. Seinen maxime utileeft.

\section{Amianton.}

Amianton, in Cypro gignitur, fimile alumini fifs fili, ufus cius ad pflotbra que exiccent.

\section{Ammos, Harena.}

Harena in luttortbus maris, potenter exiceat, fucs cenfa enimin fole exugit at q́; exiccat aquofa corpora, fi in ed uidelicet $\int c p e l$ lantur, $\sigma c d$ obruantur, calefas Ats etiam fomentum ficcum efficitur uice milij $\mathcal{O}$ falis.

\section{Ammoniacum.}

Ammoniacum, incenfum uel uapordriü, fuccus eft feu gummi, mollicndi habens poteffatem intenfam, ac perinde $\mathcal{O}$ f cirros, $\mathcal{O}$ lapidofas duricies diffoluit.

\section{Amorge, amurca.}

Amurca, fecundieft ordinis, large calefacientiums o arefacientium. Hoc nam '́; pacto artdorum corpos rumbulcera medicatur, alia uero omniacontendit atǵ, irritat.

\section{Ampeloprafun.}

Ampeloprafum, id cft, Agrefte porrum, acerris mumexiftit, $\sigma$ calidi Simum, in fupremo ordine, $S e=$ catcraffos $\mathcal{E}$ glutinofos humores, fed $O$ cacoftoma $=$ chum 


\section{DE SIM PIICI B VS:}

chum, ab obftructione liberans.

Ampelosagria, ideft, Vitisagreftis.

vitis agreftis, tam racem guàm 'ong germina ipfa, exiergendiuim habent $\mathcal{G}$ ad/trictori= un habent quiddam.

Vitis fatiua \& culta.

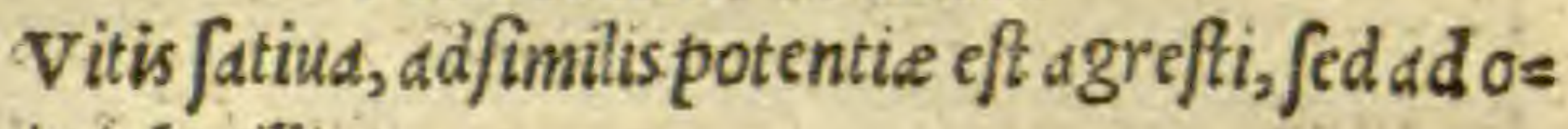
mia inbecillior.

\section{Vicis alba, Brionia.}

vitis albe, quame or Brioniam of pflotbrum uos cant, germina, quafiamica ftomacho cduntur, ov uri nam ctent - Radix uero cum boc quod abftergit, $p a r=$ tum $q_{\text {; }}$ eft tenuium, etiam mediocriter calefacit. QuL $4=$ rclienes induratos, potaliquefacit - Tum foris impos fitacum ficubus, $\odot$ p fords $\odot$ lepras fantat.

\section{Vitis nigra.}

Vitis nigra, que o ipsa brionia eft dicta, ante dis cte fimilis eft per ommia, preter quàm quod imbecil = lior eft.

\section{Anygdala.}

A mara amygdala, extenuandi, $\mathcal{\sigma}$ abobftructione liberandi uim poßtdent, corum que in inprofundore $=$ fidentlentorum o crafforum bumorum, una cum ex = tergendi cutem faculiate. Arbor quog; eandem uim babet.

\section{Amygdala dulcia.}

Dulcia amygdala, calida mediocriter funt. 


\section{P A V.L. AEIGI NETA}

\section{Amylum.}

Amylunt, fit extritico repurgato, dt $q$; dqua made. $f_{a} c_{t o}$, ea aqua fub indemutata, quinques, inde tan: dem cum molltum fuerit, per colum purgata, aqua demuo infufa. Sepurare furfures of ficcare oportebit in jole conffitum, priufquam arefcat: Sic paratum, potentiam habet refrigerandi mediocriter of defic candi, poftremo o a pera quéq, lentendio

\section{Amomum.}

Amomum, poteftate dcoro equipardtur, nifi quod acorum aridus, amomum ueroconcoqubilus cst.

\section{Anagallis.}

Anagallis, utraq; abftergendiuim fatis habet, par ticipat quiddam ettam $\mathcal{O}$ fubcalidum, $\mathcal{V}$ trabens. Itág; euellitftirpes quecorpori unheferunt, $\sigma$ éride ribus mucumemungtt.

\section{Anagyris.}

Anagyris, frutex eft grauiterolens, $\mathcal{O}$ digerens tis cxcalefactentis $q_{\text {; }}$ facultatis. Semen eius tenuß stmum nomitum prouocat.

\section{Andrachne, Portulaca.}

Andrachne, in poteftate refrigerandi eft tertie claßis, humectandi uero fecunde. Hac ratione uentri impof ita, eftuätes tuuat, fluxiones amouet. Et quia ui fcofa bumeditate predita $f$ t, dentium ftuporem $a b$ ace to fis contractum, lenut $\sigma$ extenust ${ }_{2}$ ob fuum lentore

Dyffenteris 
By $\int$ fenterias etiam o Hermorrogias refiringit. Andrafiamion.

Andrafiamton, bifurium babctur, alterum quide

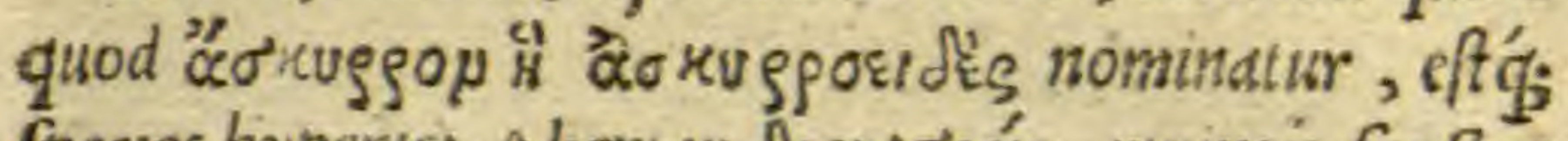
fpecies byperict. Altcrum fiourớás, utrius q; fructus Cach.rticus exiftit, folta ficciora.

\section{Androlarces.}

Androfarces, amara ov acris herba eft, digerentis - ficci temperamenti, quod sxinde liquet, quod tum bcrba ipfa, tum fructus, urinam promouent.

\section{Anemone.}

Anemone, oimnes acute or abftergendse funt po= tentic, extrabcndi quoque $v$ ord uaforum $r e f c=$ randi facultatem obtunent- Qusure pituitam proij= ciunt, o lepras arcent Cataplafmate, atq; lac tras bunt.

\section{$\checkmark$ Anethum,}

Anethum, calefact in fccundo ordine interfe, ficcat remißus. Itoq́; oleo decottum, digcrit, dolo= res amouet, fomnum concliat, cruda concoqut, tumoresque $\int \mathrm{cd}$.t. v vtum atem tertij ordinis $\mathrm{ca}=$ lefacientum, tum extccantium fit, of ob hoc ulces = ribus bumidis flaccidisq́; impofitumituat. Humidius uero minuscalidum, uiride neagis concoquit-

\section{Anthyllis}

Antby?lis duplex eft, fed utraq; bisicera deficcat. Eft etiam que chamept by fimilis oft; fed quadam tenus fubitiorum 


\section{P A V L. AEGINET A}

fubtliorum partium quàm altera. Adeo ut $\odot$ comicis albus competat. Quine ipfa magis allera extergere poteft.

\section{Anefum.}

Ane $f i f \mathrm{cmen}$, admodum acre eft , calefacitó; $\mathcal{O}$ fic cat intertiogradu, $\mathcal{E}$ perinde urinamciet, uentos $q_{;}$ diftrahit.

\section{Antirrhinum \& Anarthinon•}

Antirrbenon fiue antirrhinum, affinem bubonio fa cultatem obtinet, licet inferiorem, actenuiorem, Itág. exillo, de hoc conijcito.

\section{Anonis uel Ononis.}

Anonidis radix, maxime calefaciendo eft. Cuius fuccus potus cumuino, urinas pellit, calculos commil = nuit. Efcharas uel carbunculos erumpit, $\mathcal{O}$ dentium doloremmitigat.

\section{Aparine, Lappa.}

Aparine, quam alij phylantropum, alij omphacos edrpum, cognominant, mediocriter abfergut or cales facit - Habet autem o aliquid partium tennium.

\section{Apios, Pirus domeftica.}

pirus, inequal temperamento conftat. Pructus partim terrce, partum aquofe nature eff. Promde. efus ftomacho falubris eft, \& fitim extinguit. impo= fitusuero arefacit $\mathcal{O}$ mediocriter refrigerat.

Apocynon, Cynocrambe uel Braffica canina, uel Brafficaruftica. 


\section{DESIM PIICIBVS.}

A pocynon, funt qui Cynocramben uocant, $v$ Cy nomoron, quia cclerrime cancstollit é medio. Qutan hominibus quog; uenenuin eft-Herbd immodice foelet, callda $\sigma$ ficca, itd $q_{2}$ illita, admodum digerentis est fuccultatis.

\section{Zopiffa.}

Hypochyma, à nauigijs derafauetus pix, co quad marino falecommaduit, $\odot$ ficcandi $\sigma$ digerendi tu $\rightarrow$ mores, uim habet.

\section{Araneus:}

Araneum in emplaftrum emollitum, frontif̧; o tê porbus illitum, tertiana febris circutus finireautor est Diofcorides. Sangutnem fiftitteld etus impofita. Et bulcerum in fumma cute inflammationes arcet.

\section{Argemorie.}

Argemone, thm abftergentis, tum digerentis eff po tentis.

\section{Acifaron.}

Árifaron, aro o multo mitus, er actius eft, radice blius matore.

\section{Ariftolochia.}

Ariffolochic omnes quidem attenuantium partiums funt, abftergentes, amarulent e, $\mathcal{O}$ fubacres, $\mathbb{E}$ ad mul

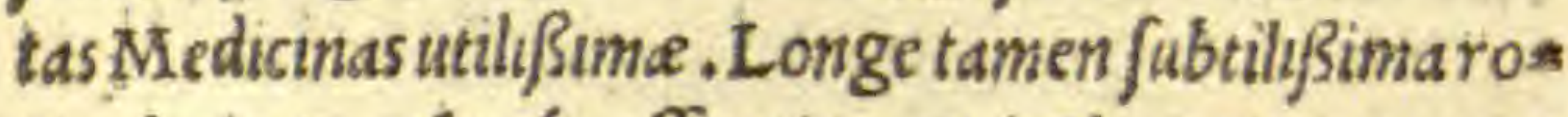
tunda, in ommbusq́; efficatior. Cui Clematites nomert eft, rcdolentior quidem, fed imbecillior, Longane ip $\sqrt{\alpha}$ quo g; mulsü cfficsax, calefacit tamé eque ut rotundior. 
\& P $\mathrm{VI}$ IEGINETA

\section{Arceuthos.}

Iuniperus arbor, calc facit, $\sigma$ ficcat, f cd in tertiadi Ptantia. Fructus quoq, arefactifimoluter, fed remifsus, atg; in primo ordine.

\section{Arcion.}

Arcion, fiue profopites, folia colocynthidi fintlis habet, fed matiora, e denfiora, firmiora qu, quétuctu= fis hulccribus impofita, digerit, ficcat atc; daffirningto Arctium.

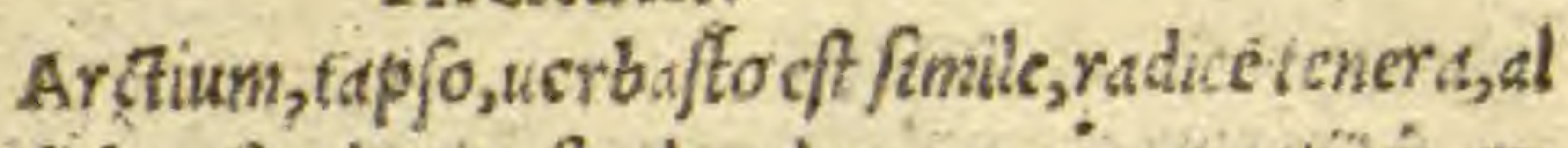

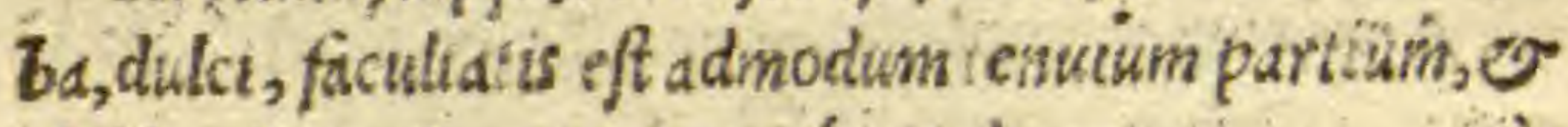
ob id exiccantis, extergentis q; mediocriter.

\section{Armeniacum.}

Armeniacum, tim habei cxtergentem cumleuiculd

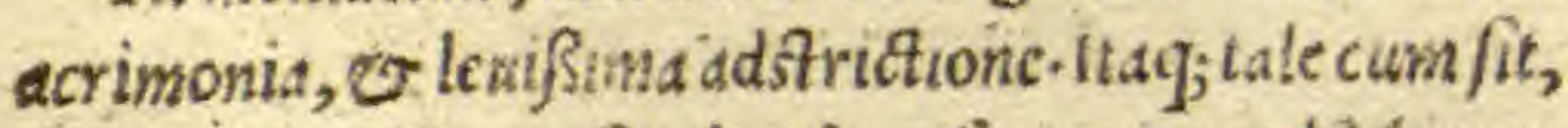
ocularibus mifcesur facultatibus, factt etiam ad priorum in fupcrcilijs incrementum.

\section{Arnabo.}

Arnato, inter aromata numeratur. Qure ungue tis piurimum mifcetur, calide of ficce potentis, $f e=$ cundumtertium ordiniem, proximumicasfie $\odot$ car= pefio. Vude $\mathrm{P}$ ofidonius, cum forte fortuna non fue= rit ad manum, cinamomo uleridum elfecenfuit.

\section{Anoglofium.}

Arnogloffum, refrigerat finul o deficcat, in $\int \mathrm{c}=$ cundo ordine, mixtum tiabens quiddam aquaum, tere reum, $\sigma$ aufterum, Ideog; aducr fum bulctrd, quee 


\section{E SIM P L I C I B V S.}

Cacoethe, ideft, Rebellia, uocantur; congruit. Item aduerfus fluxiones or putredines id ${ }^{\prime}$; temperature fymmetria Sanguinis ctiam eruptiones fiftit-Breuter, ad plurima utlle. Arefacit enim citra uellicationem, $\mathcal{O}^{\circ}$ refrigcrat. Nam ficctatem obtinet morfus expertem, o frigiditatem que nondum ob/tupeficiat.

\section{Aron.}

Aron primi ordinis eft in cale faciendo $v$ ficcdis= do. Terrena or ipfume fentid conftat, fed calida. Pro inde extergendi utm poßstdet. Radicestamen funt calis diores, er dracontium efficatius eft.

\section{Arfenicum.}

Arfenicon, facultatis est caustica, fue urentis, utun tur porro ad p filothra. Verum, fi diatius befcrit cuti, non parcel. Vifum tenuium partium redditur.

\section{Artimefixe.}

Artime fe utreq́p calefaciunt in fectundo ordine, arefaciuntóp in primo, funtơ; tenuium partium. Itaqg

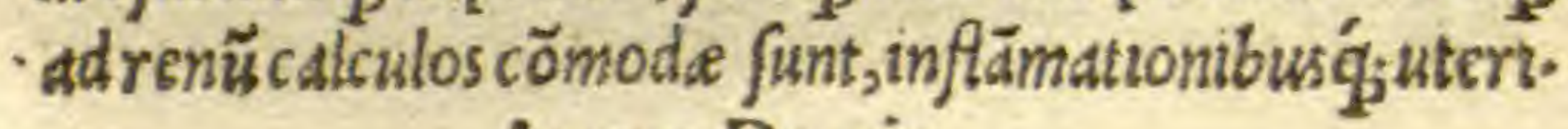

\section{Artos, Panis.}

Panisillitus, potétie eft magis digerentis quàm tri = ticü, propter naturam jalis o fermenti. Siquidem ex: tractiuum eft maldrum paßsonum fermentum:

\section{Afarum.}

A farum, acoro confimiles uires habct, intenfiores tamen paulo.

Afbole, Fuligo Piatorum :

$$
\mathrm{C}_{3}
$$

Fuligo 
$\therefore$ PAVI. AEG I NETA

Fuligo, qua picfores utuntur, quacq; ex uitriarijs officinis defumitur, digerendiutires, $\mathcal{O}$ adstringendi

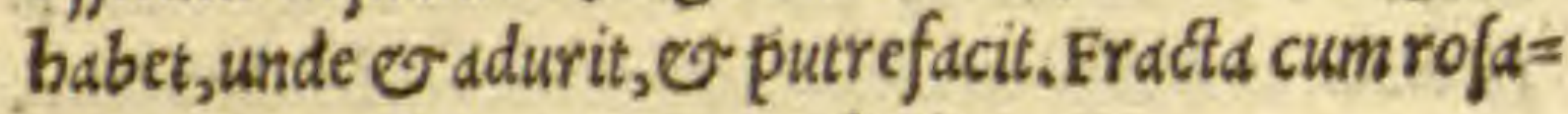
ceocerato, adcicatricem perducit.

\section{Aftalabotis,}

Aftalabotis, anumal ad allalicet inutile, tamen àf cor pione mor fis fuperpofitum, auxiliarifertur. Mifcetur

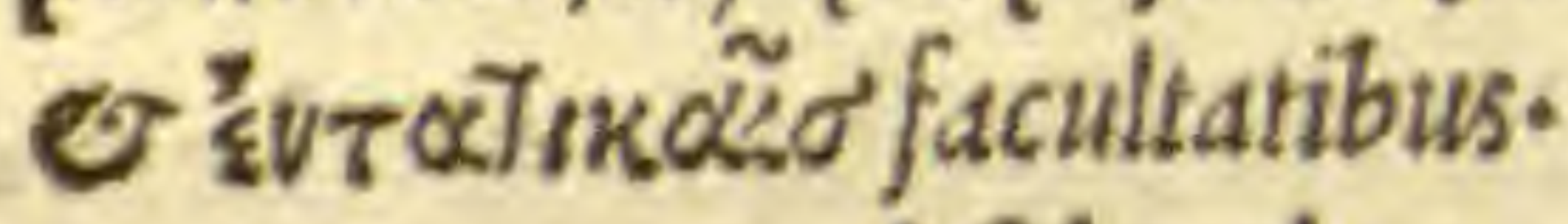

\section{Aftepias.}

Aftlepias, calldd $\odot$ ficcacum fit, partium quoq; tes nuinm, tormino is fubuenit in uinopota. Preterca illi= ta uenenatorum mor fibus, er maleficismammarum $\sigma^{\circ}$ mulicbrium locorum utcijs.

\section{De Afcyrro.}

Afcyrron, in Androfemodiclum eff.

\section{Afpalatos.}

A/palathos, ex dißimilium partium potentijs con= ftat, acutis, 0 acerbefcentibus. Itaq́; utriufq; ratione dcffccat, et proinde ad putredines et fluxiones eft utilis.

\section{Afparagus.}

A Paragus Petreus, tiel Myacanthinus, absiergëdi

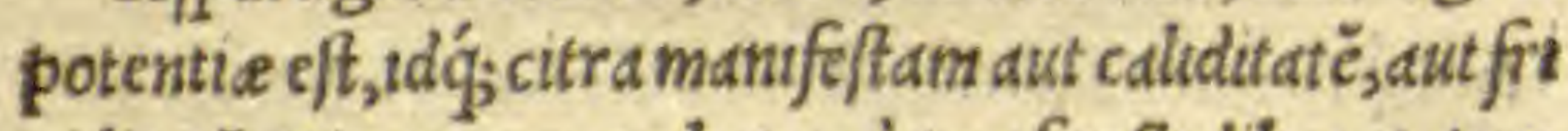
giditate. Hinc renes o hepar à prof farculiberat, tum maxime berbeipfius radices of f $\mathrm{cmch}$. Dentiü quoq; dolores fanat.

Afplenion.

A Plenium, tenusum quidcmpartium, nontamenca lidaherba 


\section{DE SIMPLICIBVS.}

lidaherba eft. Hac itaq; ratione deficcat, ev proinde. ad putredines er fuxiones eft utilis.

\section{Aftaphis Vua paffa.}

Aftaphis, culta quidem coquendi, adftringuendio leuiter digerendifacultatem $p o \beta$ idet, at agreftis uehe $=$ menter acrem obtinet, adeout ex capite purget, quod

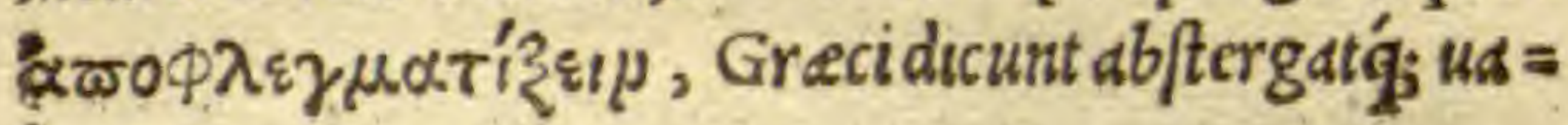
lenter.

\section{After Atricus, Bubonion.}

After Atttcus, alij Bubonton uocät, non ob id tant $\bar{u}$ quod llitum, fed ctiam quod fufpen fum bubones feruas recreditur.Mixla potentie $\mathrm{c} f \mathrm{t}$, digerentis utdelicet, $\boldsymbol{\sigma}$ refrigerantis.

\section{Aftragulos.}

Aftragulus, paruus breuis q; eft frutex, radices has bens conftrtctorias. Quamobrem etiant ex natura ef: non inftrenuc exiccantium. $\mathrm{Nam}$ bulcera uetera glutie nat, $\mathcal{O}$ aluum fluxis tentatom fiftit, fi quis in uino de coctam radicem bibat. Plurimus huius fructus prouen= tus eft in Phenco Arcadice.

\section{Afphaltus. Bitumen.}

A phaltus, ficcat ef calcfacit in fecuido ardine. Pro

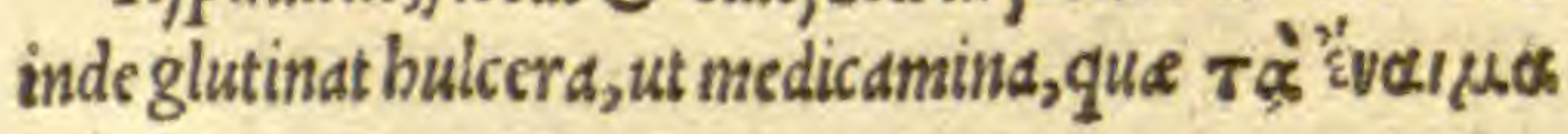
uocant.

\section{Afphodolus.}

Afphodeli, radix abftergendi $v$ diffrahendihabet potenuam . Crematis uerocinis caldior $\mathcal{V}$ exiccdntior, 


\section{$P$ A $V$ L. A E GINET,A}

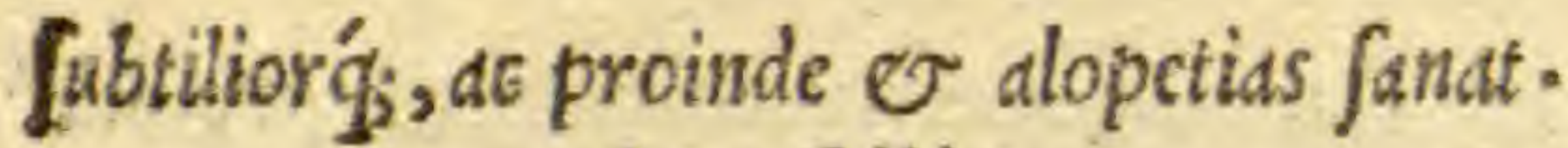

\section{Atractilis.}

Atractilis, fecundieft ordinis bumcefantium, primi seroxefrigerättum. Quddere uentrem celcritcr pers meat. Sementamen abftergentis nature eff, 0 arqud $=$ tis opilationemhepatis aperit.

\section{Aphace.}

Aphace, uim habet aftringendi, quemadmodum $v$ ipfa lenticula, edendáq; eft, ut lens, fcd agrius concoqui tir, etiamfideficcet ualentius, cum mediocricalore,

\section{Aphonition.}

Aphonition, diffrabendi e digerendipartium, for dida queq; repurgare, $\mathcal{E}$ pruritus fedars idoneum,po tum tamen, cacoftomachum eft.

(2.) . Aphronitron.

3 Nitri puma, equalcm cum nitro potentiambabet, partiǘq ; eft magis tenuiü, afpectu tenui, farine fimilis. Achras, pirum agrefte.

Pirum agref $\tilde{c}_{2}$, proximä piro facultatem babet, fed fedadftrictiorem, ob boc uulnera conglutinat matiord. Abfynthium.

Abfynthium, calidūeft tin ordene primo, dridü autems scrtto. Adfitrictoriam $\mathcal{E}$ amaram $\mathcal{O}$ acrem $\mathrm{fimul}$ qua litatespofidet, propter quod bilio fos uentris humokes per cgeftione inf crnam propellit, dut purinas euacuat. Ozabus anté cotenta in uétre pituita eff, propter aftrti=

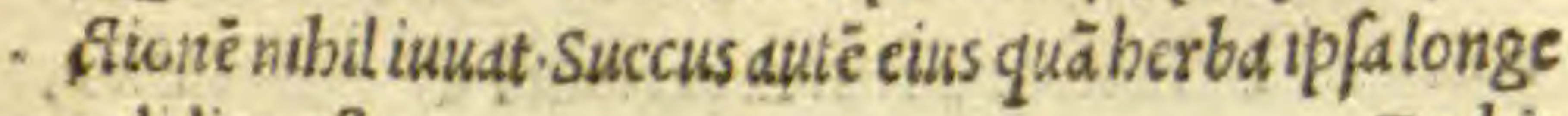
calidior cft.

Debis 


\section{DESIMPIICIBVS}

\section{DE HIS Q V AE AB B}

litersuncipiiant.

Balamus Myrepfica, id eft,

Mirabolanum.

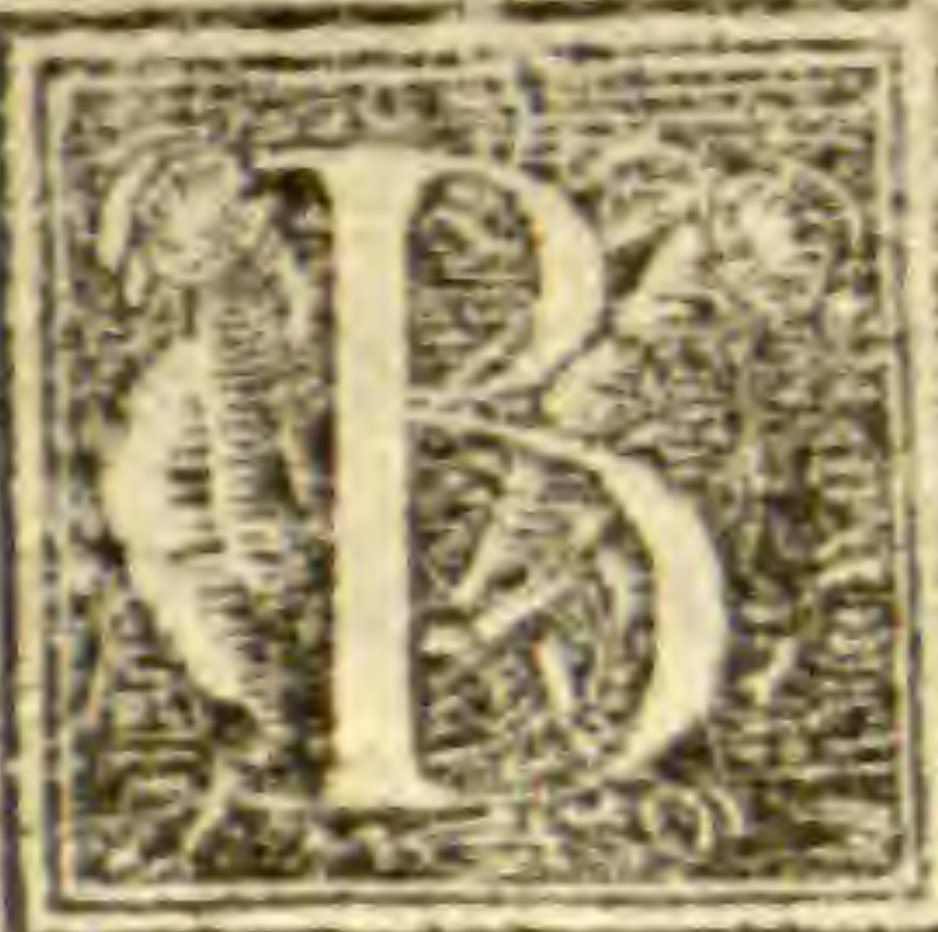

Alamus Myrcpficd, quod interne bde bet carnofum, abftergendi $\sigma$ fecands uimbabct. Quod fi quis cum melcrato bibertt dracbme ponderc, facíe tomu crit. Aluum etiamper inforna large de ijctt. Cum oxicrato intefinorum pref frailum medcturExtcriora quoq; cum aceto perunctd, fanat, nimirum propter abftergendi utm quam babet. Cortex quoqs cius admodum adftringit.

\section{Bacharus.}

Bacchar, fruticofa eft her ba, cimnamomo proxima, coronaria, acres. Huius radix decocta, ipertiua eff, uris

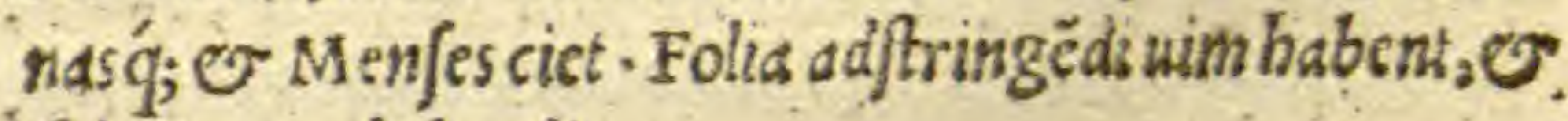
fiuxiones cohibendi.

\section{Balauftium.}

Balaufium, flos cft agreftis punice, immodice dds Atringit. Alq; eo fane nomine uetera bulcers ad dicatri cem perducttodeftillationes $\hat{q}_{\text {; }}$ fiftut.

\section{Balfamon.}

Bd] fumilignum dcficcat quidem, er calcfacit, feckn

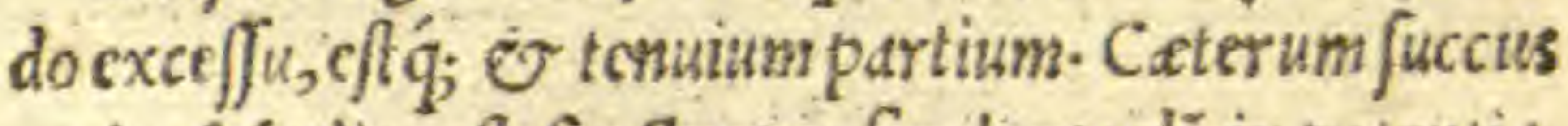
multo fubulior eft, fructus perfimilis quidé in potentid, fedlongé infrior tenutatis.

$C_{4}$ Bslote 
PAVI. AE G INTA

\section{Balote, uel Marrubrum nigrum.}

Marrubrum rigrum, acris $\odot$ detergentis of $p o=$ tentis-impo fitum igitur emplaftrimodocum fale, rabi di canismorfibus auxiliatur.

\section{Batus rubus.}

Inrubo, quod frutico fum exifttt, qualitate adftrine gente participat - Q euo fit ut uulncra glutinet, fluxio: nesof; fiftat-Radix uero, acerbef ccit feruentius, tenuiü $\dot{q}_{\text {; }}$ partiü eft magis, $\sigma$ pinde in rembus calculos atterit.

Fruclus, $f$ if it pramaturus, moderatius calefacit $\sigma$ ftringit-Quamobremciboidoneus eff. Immaturus ue:

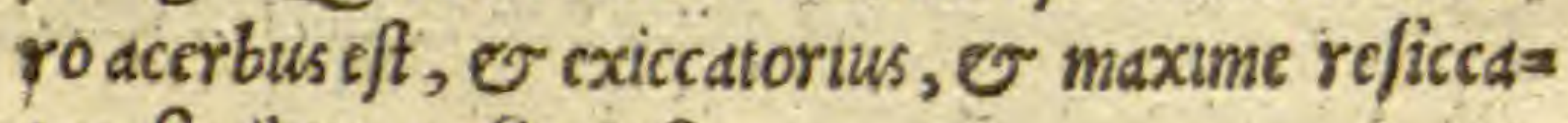
tus, fomilter o flos ipfe.

\section{Batrachion.}

Batrachion, quatuorhabet differentias, omneis ue= rocalide of ficce, uehements funt potentie, acute, uidelicet $\sigma$ cauftice. Adeout cum dolore cxulcerent, quibustamen fi quisutatur moderate, exteriora tan= tum, qua in eute accidunt, abftergit · Radix ueropro= pter ficcitatem fternutatorium eft medicamentum.

\section{Batrachi, Ranæ.}

Rane in iufculo cocte, a beftia mor fis commode eduntur. Porr o uftorumcinis, quoniam ficcißimus eft, cumliquida pece hemorrogias fiftut, et alopecias curat.

\section{Bdellium.}

Bdellium, tum Scythicum, tum Arabicum, ddmodü emollentis eff facultatis coruminge funt indurata, mas= 
xime uero quod recens fuerit. A rabicun diureticü eff, ordigeftiuum.Vnde calculos attertt.

\section{Betonica.}

Betonica, ualde cxalibus ramulis ef therba proximas pulegio frondcs babens, fed tenuiores, VQy $g \delta^{1} 0^{1} \mu \alpha=$ Tora jevoúvyoro Seritur plurimum in petrofis locis, qua ustuntur ad renümedicamina $\cdot \mathrm{E} / \mathrm{t} \sigma \mathrm{\text {Romanisalla }}$ quedä Betonica dicta, quä Diofcorides Ceftron nomi= zuat, alij uero $p$ fichotrophö.P ropterea quod frigidis to cis plantarigaudet, mbil fimile haběs iam ditte, preter efficatiam, ad ducendā eni urină utuntur $\sigma$ alis multa conducit nibil eque.Radix cius cum bydromelite pota, uomitumciet, $\mathcal{V}$ folid uentrem fubducunt.

\section{Bechium.}

Bechion, quod alij Bechicon, inde ddeo nuncupatŭ eft, quod Tufes orthopnoeds iunet, fuligine eius in $p$ i= ratuattracto, Coalitum $\varepsilon$ t, excalida $\mathcal{O}$ ficca fubftatia.

\section{i Blitum.}

Blitum, holus eft c fculentum, temperamento humi= dum or frigidum, in fecundoconftitutum ordine.

\section{Bulbus.}

Bulbus, ille, qui eft efculentus, amaritudinisq́; $\sigma$ ad fristions partsceps eft aliquantulum: vulnera conglus tinat $\mathcal{O}$ abftergit.

\section{Bulbus uomitorius.}

Bulbus uomitorius, multo calidioris eft tęęerature. Botiys.

C 5 Botrys 


\section{P A V I: AE G IN T A}

Botrijs, quam alij ambrofiam, alij ueroartemifiane rocant, p'anta uino fa ac fragrans manifffe. Cumbuino pota, orthepnoicis remedio eft.

\section{Bubonion.}

Bubonion, in Aftere A tico diclume/t.

\section{Bugloffum.}

Buglofjum, bumidum o caldidum eft temperamens to.Itaq; uino imm Ifum, latitiam ac hylaritatcme effices redicitur.

\section{Bunion.}

Bunion, fue Arction, calldume $f$ t, $v$ urinam amoz uet, $\odot$ - Menfes puocat. Cuifimile eft $\mathrm{O}$ p feudobunion.

\section{Bupreftis.}

Bupreftis, animalculum, Canthtridiper omnia $f i=$ mile, pro qua 0 in medicaminibus fupponitur.

\section{Butyrum.}

Butyrum, cöcợuendı $\odot$ medıcriter digerendipo tentie, in mollibus corporibus. Bubones igitur \& paro tides curat, cicatrices attenuat, $\odot$ maxime dentü dos lorens, quem infantes fuftinent dentientes, mitigat. Ad hecin peripneumonicis effectibus in eclegmate proficit.

\section{Buphthalmon.}

Buphthalmon, fimilem habet Chamemelo flore, fed multo maiorem tum acriorem. Proinde $\mathcal{V}$ digert . Adeo ut $\mathcal{O}$ duritis fanct cerato mixtum.

\section{Brathi Sabina.}

Sabus, Cyparißo equipollens, ca'rfacit of ficcat in tertio 


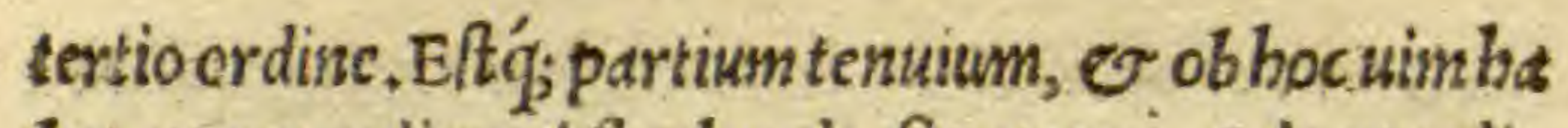
betattcnuandi $\sigma$ diffrabend, fi potetur. Ad putredi= nes, perinde ut cupreffus, accommodari poteft.

\section{Britannica.}

Britannica, adf tringentis pariter $\sigma$ conglutinan= tis, eft temperiel, figura fimilis agrefti Lapatho - Euus fuccus in uentriculoputredines fanat.

\section{Bromos.}

Bromos, funilemordeo uim obtinet. Nam deficeat , $\sigma$ digerit mediocriter, fiue mordicatione. Adftringit quoog nombihbl, qua fit, ut uentris diarrhocas perfanet.

\section{Bryon Thalaffion.}

as Fryon Thala ßion, refrigcrat 0 ad/tringit. Inde im pofit sam feruentes pblegmonas iuuat.

\section{Bryon Sphalanchnon.}

Bryon Sphlanchnon, quod in quercubus $\sigma$ populis - dibis o piceis inucntur, diftrabendif fimul o mollien di babet potefitatem mediocriter. Precipue quod cedris \& adheret.

\section{Brionia,}

De Brtoma dictum eft un uite alba.
DE H I S
Q V AE INCIS piunt abltera $\mathbf{r}$.

\section{Delacte.}

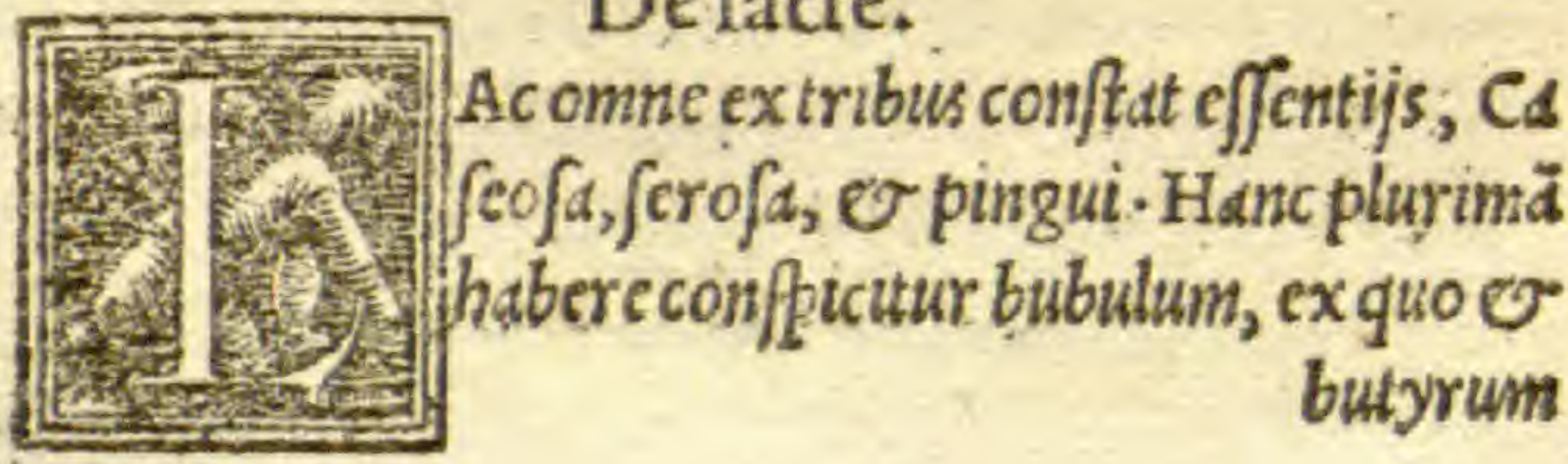




\section{P A V L AE G IN E T A}

butynum conficitur. At ferum ipfum extergendi ha s

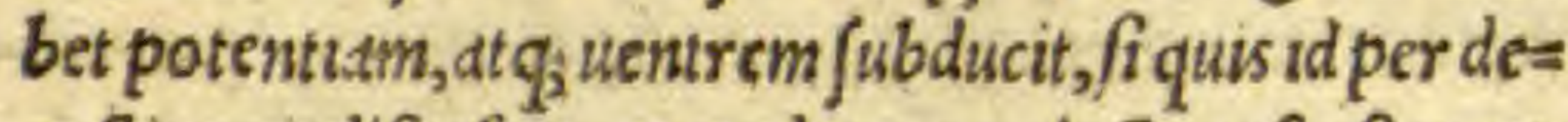
coctionem dif $\beta$ efcat. Quod uero ex lacte cafeofum er denfum ine f, magis emplaftratur, $\mathcal{O}$ cóm primis no= mine quod acrimoniam hebetat.

Decoctum autemlac $\delta 1 \ddot{\alpha} x \alpha \chi \lambda \lambda \hat{\kappa} \tau \omega p$, uclaliter, quodammodo optimum fit dy]fentcrie, e corum que in uentre funt acrium fluxionum. Porró, lac totum ad acres oculorum fluxiones $\mathcal{O}$ alias acrimonias commo dum eff. Temperatißimumlac mulicbre, mox caprinü, dsinde d fininum $\sigma$ ouithem, ad poftremum bubulum.

\section{Muiftela.}

Muffele combufte cins, uebementcr digerit - Cun aceto igitur illitus, podagricos $\sigma$ artbriticos iuuat, are factus uerocomitialtbus prodeft.

\section{Galion.}

Galium, inde adeo nomen fortitŭ eft, quod lac cods gulet-Adfimilem Apparine facultatem obtinet, $c x=$ tccatoriam o fubacrem. Flos cius Hemorragie 0 am buftis medetur.

\section{Galipfis uel Galiopfis.}

Galipfis, funt quigalibdolon appcllent. Vrtice fimi lis herba eft, matorem tamen babent follaleuorem $\mathrm{E}$ odorem graucm, dcfijcieridi $\sigma$ mollicndı duricies, po tchtis. Facit $\mathcal{F}$ ad ca qua pafcendo ferpunt bulcerd, emplaftrimodoimpofita. 


\section{DE SIMALICIBVS:}

Garus, facultatem babct multum calidam of ficcä, ac perinde co quidà ab extra ad hulcera utuntur putre=

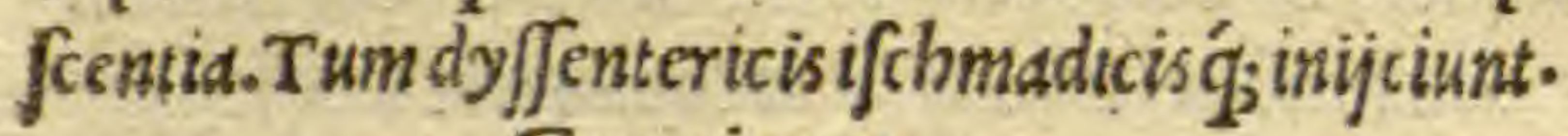

\section{Gentiana.}

Gentiand, radix efficax cum opus c/t extenuatione, purgatione o obftructionis liberatione.

\section{Geranion.}

Geranium, unü quidem folid Anemonic fimilis hd* bet, radicemef fii idoneam. Hec drachme pondere in sino pota, muliebrium locortm inflationes folutt.Atte: sumnullum babet in medicind ufum.

Terra.

Terra, qualibet exiccat, immifte, alterius fubftan: tie, of fine morfu eft . Quod fi etian ignea quedam ithiuis admixta fuerti, $\sigma$ hanc abluta amuttit.

\section{Terra culta.}

Culte terre ommis pinguedo utilis eft, ad onniuns partium, que po flunt areficri, curam.

$$
\text { Lutum Aegyptium. }
$$

I uto egypto utuntur adintcrcutaneas or plenc: ticas affectiones, ơ ad adimatum quog; mothicies.

\section{Lemnia terra.}

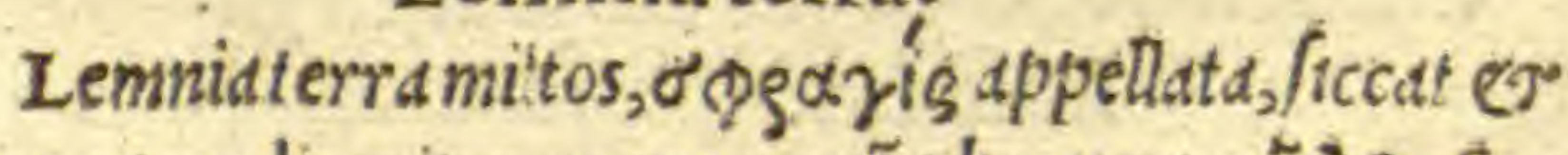
fringt mediocriter, uenenatorü pharmacor ũ ' ${ }^{\prime} \lambda \varepsilon \xi \xi l=$ rasia. - anat e hulcera cacoetbe, cum accto uel uno. Ad hae $\mathcal{O}$ Hemorrogias $\mathcal{O}$ dy ffenterids reftringit, Quin o nomas fanat, melicrato prius inf $u f a$, deinde neromuria.

Sinopis 
P A V L. AE G IN E T A

\section{Sinopica, Miltos.}

Sinopica, Miltos, acerbior Lemnia- Emplafticis mo feetur facultathbus: Pota'ǵlatos lumbricos conficits.s. :

\section{Samia terra.}

( Samiaterra, Lemnia multomitior, echicolla $q_{\text {, }}$, cuius ufus eft, vbimitigare que bumidiora funt of laxiord, uolemus. Et fanguinis quog; sputiones fiftit pota.

\section{Selufina uel Chia.}

Selufinderra, Chia, abften gentior eft - Quam= obrem quedam mulieres ea utuntur ad faciem, quod arcfaciat, $\Theta$ abftergeat, $\odot$ perinde hulcera incarnat, o ad cicatricem perduct maxime uero ambufta.

\section{Cimolia.}

Cinnoliat terra, mixte of t potentia refrigerantis fi $i=$ licet $\odot$ digerětis, illota-Abluta autem reffrigerat ualen tius or ambufta cum oxycrato medicatur.

\section{Ampelitis.}

Ampelitis, fic dicta quod germinantibusuitibus iltis

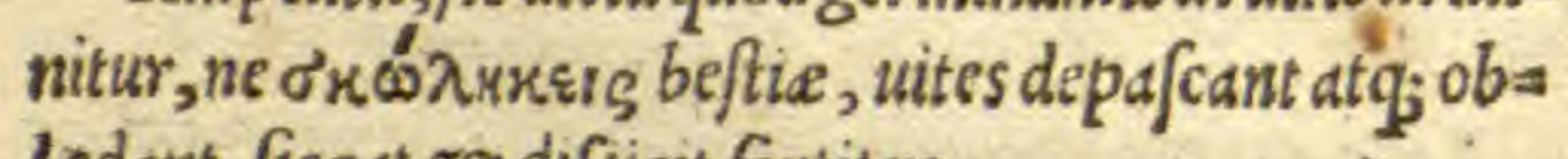
ledant, ficcat e dify cit fortitcr.

\section{Cretica terra.}

Creticaterra, aérofa, finc mor fu abftergit.

Eretrias.

Eretrias, ufta manufeste adffringit, ficcat uero fine wordicatione Redacta tamen in cinerem melior eft.

\section{Pęgnitis.}

Pegnitis fimilem habei Cimolie facultatem. Ef t tas men nigra. 


\section{Armenia.}

Armenia, quam Bolum uocdint, fupramodum exict cat. Quareauxulatur tormino/is, fanguins fluxibus; - fputis, $\mathcal{O}$ Tabi, $\mathcal{O}$ difpinoce, proptcr bumtdtaté. Pretcrea hulcer bus bumidis, o peftiferis affictibus inagnifice auxuliatur. Bibttur cum uino tenul, immixio, aut fi febriat a ger, cum aqua. Simlem Armenie habet poteftatem, qua Ochra nocatur, potentie digerēdi, putrefaciendl. Quo fit, ut admixta ceratis, carnes hulce rifuperexcrefcétes cohibedt, of uacua quo g; repleat.

\section{Senecta Anguis.}

senecta ferpentis, tuebeméter exicedt, cum aceto igi tur elixa, aurium doloribus prodeft.

\section{Senecta Afpidis.}

Apidis exutile, trite cum molle, at $q$; oculis inunde, *afum recupcrant.

\section{Vermes Terreni, uel Lumbrici.}

Inteftina terre contrita, nerisis ós impofilapracifis, conffltm o mirabliter iunant.

\section{Gigarta.}

Gigarte, arida quidem eft, in fecunda diftantia, fri= gida ucro in prtma Itáq; frigiditate fua omntbus uen= tris cü profluuio inunctis affict ibus, impendıo cöfert.

\section{Gingidium.}

Gingidiü, calidttatẽobtinet, $\int \mathrm{fdnŏ} \mathrm{admodŭ} \mathrm{manifyftă.}$ Arefacti autcm in fecüda diffantia, $\mathcal{O}$ adjftringit,ftoma chosmiction. Glaucum 


\section{- PAVi. AEGINETA}

\section{Glaucium.}

Glaucium, acerbefcitcum quaddm infusuitate, Cas terum frigefacit manufefte, adeout igni facro opemfes : rat, quitamen ualidus minime fit.

\section{Glaux.}

Glaux herba, caldda $v$ humida eft temperamento, quare lactis generandi urres habet.

\section{Glechon, Pulegium.}

Pulcgium, calefacit $\odot$ attenuat uchementer, quare ithitum rubificat . Et ex thorace, pulmoneq́g, humids, craffa, glutino $\int a \dot{q}_{\text {; facitexpuere. }}$

\section{Sordes balnearum.}

Strigmenta, ucl fordes àbalncis, calefaciunt medios criter, molltunt at $q_{\text {; }}$ difcutiunt. Ad fedis rimas or con= dylomata illinuntur.

\section{Glycyrizon.}

Glycirizd, tepide E humide potentie eft, cum qua dam tamen acerbitate. Proinde afferitates lenit, non modo arteric, uerumetiäucfice fcabré. Siti quoq;con gruens medicdmentum.

\section{Glycyfides.}

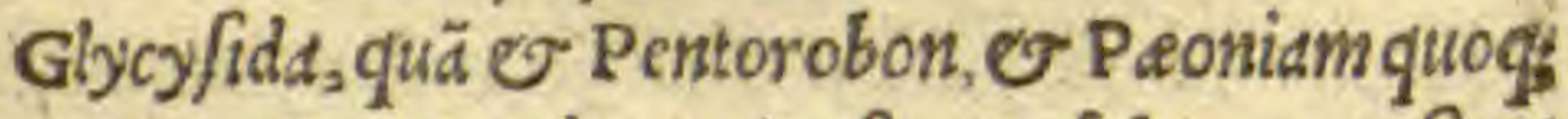
nominant. Huius radiscacris eft, $\mathcal{O}$ fubamard, ficcd quoq;, * quoda'mtenus Ed/tringens. Mcrfes tgiturc $i=$ et, renes $q_{;}$o hepar repurgat, amy gdale magnitudine in uino potd. Qubod fi austerum fucrit uinum, in uentre

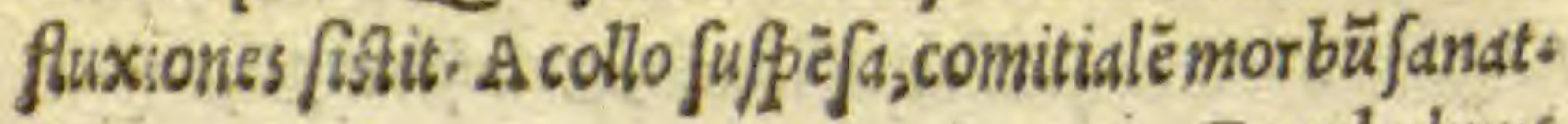
Graphalume 


\section{DE SIMPLICIBVS. Gnaphaliun.}

Gnaphalium, inde appellatū eft, quod folijs eius mol Libus, protomento utantur. Sunt duté candida o mo dice adfringunt - Ac proinde ad dy J fentcriar cum uino quopumaufterobibuntur.

\section{Gongylis.}

Gongylidis, $\odot$ radix $\odot$ femen uentos cient, $\odot$ fe= menhunanum adaugent, adcóg; $\odot$ Veneremextumus lant:

\section{Gorgylon,Sefeli.}

Gorgylon, id quod e st fli quog; nocatur. Calidu of to dureticum, atq́; ob id NAenfes ducit-Porrò radix in eclegmate, cum melle tincts, thoracis utidemendat .

\section{Gyris, Pollen.}

. Gyris, cum dimylo potêtum equalem hebet, preter quàm quod calidior eft.

\section{Gypfos.}

Gyp fos, uel Gypfum ad exiccandum tim emplafti= cam admittit:Hemorrhagids itag curat, dilutum cum albo oui liquore. Ceterum combuftum non equeeme plaftcum eft, fed ualétius deficcat, \& repercufforium eft, maxime cam oxscrato.

\section{DE HIIS QV AE IN=} cipiunt ablitera $\Delta$.

Dades. 


\section{$P$ A $V L$. AE $G I N E T A$}

Dades, babent quiddam acerbum, nonmbil etiam concoquunt $a^{2} q_{j}$ abjtergent Cumaceto coct $\varepsilon_{y}$ dentum quoq; dolores una cum acettcollutionc lenunt- Qugod /i

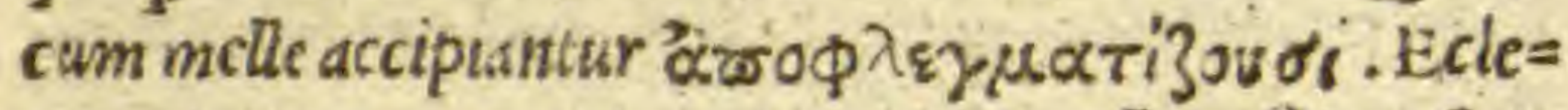
gmatis mixte, qua circa thoraccm craffirefident flui= da, a $q_{j}$ ductilareddunt.

\section{Damalcena.}

DeDamafcenis in coccymelis dicetur.

\section{Damafonion.}

Damafonion, alfterforiä facuitatem obtinet, qua na mirülapilloscöminuit

\section{Daucus.}

Dincus, qui o flaphlinus nuncupatur, duplicx est. Primiquidemradix, quit flatuofacf, Venerem prorts tat. A grcftis planéexfuffltaoris cxpers eff. Ac prom= dc urutam Menfes $q_{\text {; cict }}$ : odem modo o herbaipfa.

\section{Daphne.}

Daphnes arboris folid arefaciunt, $\mathcal{O}$ uehementer calefacunt, plus etiam fructus. Verum radicis cor= tex, amarus of quiddam habens acerbi, calculcs con= frungit. Et bepaticos unat, bibita in uino odorato, triobolari pondere.

\section{Daphne herba.}

Daphnesherbx, quam o daphnem Alexindrinam

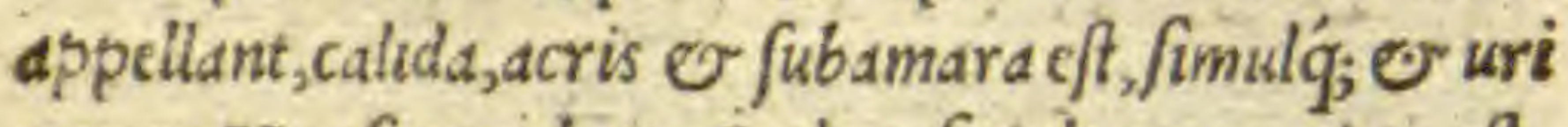
nas $0 \mathrm{M}$ enfes protictit. Eadem facultatc predita eft, 


\section{DE S I M P L I C I B V S}

que Daphnoides e Chamedaphne à nonnullis nocas tur, qua cdi etiam affolet.

\section{Pellis Ouina.}

Pellis ouna recens detracta, circumpofit d g; i ijs, qqub quocumóg, modo fuerint uerbcrati, mirabiliter adtiudt. Pellis hippotami.

Pellishippotamulith, o cam aqua fuper impofita tuberadipergito

\section{Pellis Viper $x_{\text {. }}$}

Porró Vipereintrita $\sigma$ Cataplafmata,portentofe in alopecia pilos herere facit.

\section{De tieteri calciamentorum corio.}

Veteres calciamentorum folee, ficrementur in fic= cum cinerem refoluuntur, quex calceis pedum attri $=$ tus medicatur, nifi forte fuccenfa fuerint, dut dmbufto*

Dictamus.

Diflamon magis tenuium partium eff pulcgio, ces tera illi fimile. At quod uocatur p fendodictsmum, imbs cillus eff.

\section{Dionifias,}

Dicta eft in Andrafemo.

Diphriges.

Diphriges, mifte potentie. Habet enim in fe quids dam mediocriter adftringens, o mediocriter acre: Quamobrem hulcerum rebellium falubre medicames c/5.

\section{Dipfacon,}




\section{P A $\nabla$ L AEGINETA}

Dipfaci,pine radix, in fecundo ef entium. Habet etiam quiddam abfergens.

\section{Dorycnion.}

Dorycnion, fimiletemperamento eft papatuerie mädragore, immodiccéq; rcfrigerat. Qusmobrem pal Iulam quidem ftupefaett, plufculum uero fumptum in = terimit.

\section{Draconcium.}

Draconcium, quiddam aro perfimile habet . Sed acrius eft aro, amarius, calidius $\odot$ mag is partium te = nuinum-Habet enim leuiculan quandam affriftioncm. Itág; radix pota, inteffina expurgat.Hulceribusetiam que xarox́trappellant, optimum eft remedium . Folia ad eadem pollcnt. Fructusualcntior eft.

\section{Dryopteris.}

Dryopteris, dulcis, acris, o fubamara eff, id cff, mifte qualitatis . In radice uero ttem acerbam potent $i=$ ambabct fcpticam. Proinde etidm pilis glabram cutem efficit.

\section{Quercus.}

Quercus, $\mathcal{O}$ folla $\odot$ frutus ficce potentie funt, in genere falliceteorum, qua tepida eftimantur. Ideo bukcerarccêtia glutinare $\&$ pblegmondsuncipientes fa nare creditur. Ceterü quod fub putamine glandis mem brane fimile, tum quod fub glandis iffuscaliculolatet, ca uidclicet que fructus carnem contueftit, odftringen= 


\section{DESIM P LICIBVS.}

tiuseft, ad proflunium mulicbre, or reliquds quoque fuxiones datur.

\section{E H I S $Q V A E \quad I N=$ cipiunt $a b \mathrm{E}$. \\ Ebenus.}

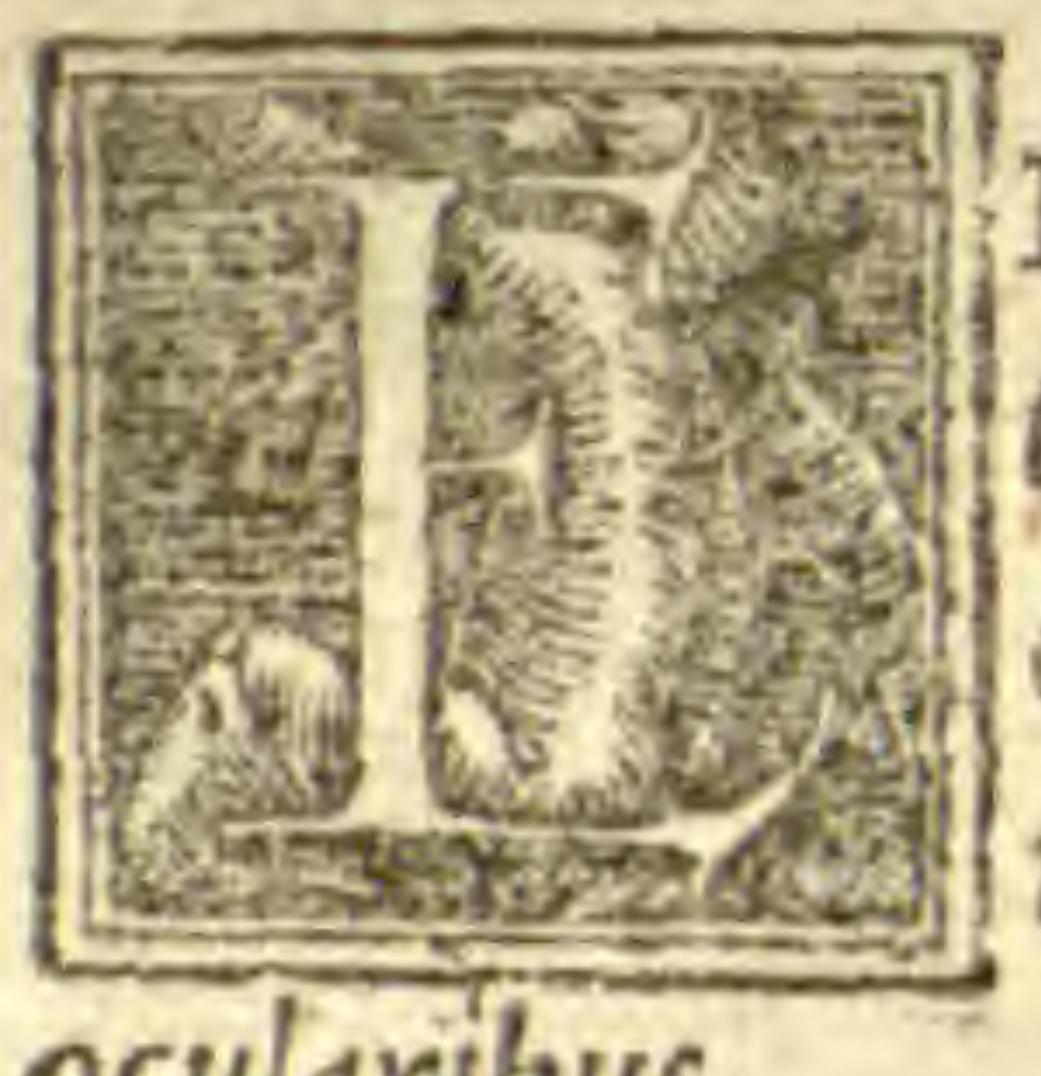

Benus, lignumcalide eft o obftergen tis, partium'; tenuiüpotentie - Quam obrem que pupillas obfufcant, exicrge re creditur. Mifcetur $\mathcal{O}$ alijsrcmedijs ocularibus:

\section{Cerebrum Leporis.}

Cerebrum leporis coctu attritum ; o efum, pues ris dentientibus uttle effe ferunt- Quidametiam aducre fus metum uthliter edi cercbrum hoc prodidere.

\section{Cerebrum Galli,}

Gallinaceorum cercbrum, ut inqut Diofcorides, da tur in potione cum uino contra uenatorŭ mor fus. Come péfcis idem ex cerebri mëbranis erumpentë fanguinĕ. Cerebrum Cameli \&ZMuftelæ.

Cerebrum Cameli, autorc Galeno, ficcatum, in aces to ó; propmatŭ, Epilepticos fanat. Quod idem $\sigma \mathrm{N} u=$ ftele cercbrum preftare dicitur.

\section{Cerebrum Chelido.}

Hirundinum cerebrum cum melle mixturs, oculor fuffufiones, compelcit.

\section{Cerebrum Ouillum.}




\section{P A V L AE GIN E T A.}

Cerebrum ouitlum praparatum, codem modo quo leporis cercbellum, puerorum doloribus, quem dentien do patiuntur,accurate medetur.

\section{Olea.}

Oliue ramiquantum babent adftrictionis, tantum - frigiditatus partictpes funt . Fructus uero, fiquidem ad unguem maturuit, moderatecalidus eft. Sin immatu rus, tum adftringit tumrefrigerat.

\section{Olea Aethiopicalachryma.}

olce Ae!hiopucelachryma, fimulis Armeniaco tenel 10. Adftergentis ef potentie, unde contracicatrices, al= bugines, $v$ bebetcs oculos, ip fo utuntur. Itemad den= tium dolores, quorü cauis oportet tmmitterc. Sunt qui bincagreftcm oledm effe uelint.

\section{Oleum ex drupis.}

Olcum exdrupis dulce, o calidü eft mediocriter, fedmagishumectorium.

\section{Oleum ex crudo Omphacio,}

Omotribes, quarto plus adfiringt, tanto stiamma gisrefrigerat.

\section{Oleum ablutum,}

Oleum lotum minzmc omntum mordax $e f$.

\section{Oleumuetus.}

Vetus o'cum, quanto antıquius, tanto calidius fit. Po te t or bus fु; ad cuaporandum uribus, muxime fite= nuiumptrtum d proncipio fuerit. Huiufmodefere bs

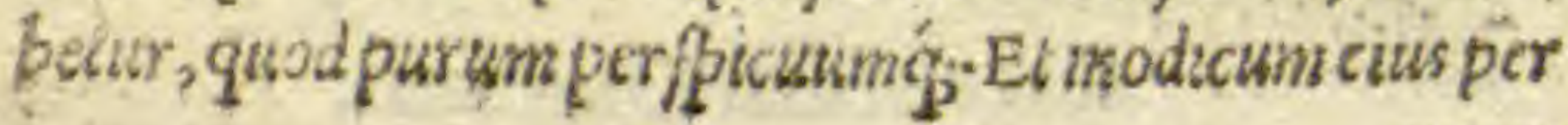

cuicm 


\section{DE S I M P L I C I B V S:}

cutem iniunctum celcritcr d cutc unbubitwr, qualc eft Sabinum.

\section{Oleum agreftis olę:}

Oleaffri func agreftis olecolcum, ablergentis fo mulev adfringentis potcntie eft, fed ommum plane fquallidisimum.

\section{Oleum Cicinum.}

Cicinum oleum, quä fimilimü olco cxoliua uetufta. Sefaminum Raphaninum.

Ceterd olca, fcilicet Raphaninum, Sc faminum, O / que funt alia, procuiusq́; natura, ex quibus funt, uaris antur or potentiarum differentias fortiuntur.

\section{Oleomel.}

Oleomel, in Syra ex arboris truncomelle cra Situs odu'cius fluit, quod potum beminarum duarum pons dere cum aque bemma una, per alsum cruda o bilofa trabut. Veruntameri quafi ueternofi torpent tdaccipi= entes quod ttmere non oportet, fed excttandi fuit.

\section{Elateriuth.}

Elaterium fylueftris cucumeris fuccus. Fertur $\int e=$ cundum obtinere ordinem calefacientum. Menfes pro uocat, $\mathcal{O}$ factum intcrimit appofitum, propter immo $=$ dicamamaritudinem Euacuat $\mathcal{O}$ pornarescumlacte inftllatum. Purgat per inforna phlcgma - Intcrdumáq; o fanguincm. 
PAV L. AE G I NET A

\section{Elate.}

Elatedrbor, calcfacit 0 ficcat, modo quo 0 popu lus. De Rhefind eius, in Rhefinis dicetur.

\section{Elatine.}

Elatine, herba eft perfimilis Helxine, mediocriter tum rcffigcrat, tum adfringit.

\section{Elaphobofcos...}

Elaphobofcus, impenfecale facit of fubftringits Elenium, Enula Campana.

Elenium, calidü cft of ficcum, cum humiditate inci= dere ualcnti. Quapropter eclegmatis mixtum, craffos extborace humores educit - Rubbficăt eius quoq; uipass tes, frigidis ac diuturnis uexatas affectibus.

\section{Eleofelinon.}

Eleofelenon, quodin aquofis locis prouenit. Eddem poteft, que bortenfe $\mathrm{O}$ domefticum Selinon, licet ma $\Rightarrow$ sus co fit.

\section{Elephas,}

Ex unguibus Elephan is ramenta cataplafmata $\mathrm{Pa}$ ronycbias fanani. Ceicrum que ex o $\beta$ tibusetus funt $\mathrm{O}$ dentibus, ficce atq; ab/tergentis funt potentie, atque eiufmodiquog; mijcentur med.cints.

\section{Helcyfmata,}

Heliyfma, argentucft fcoria, molibdene confimi= lem facultatem habcns. Strit git crim fimulaíg; euclltt. Quare 


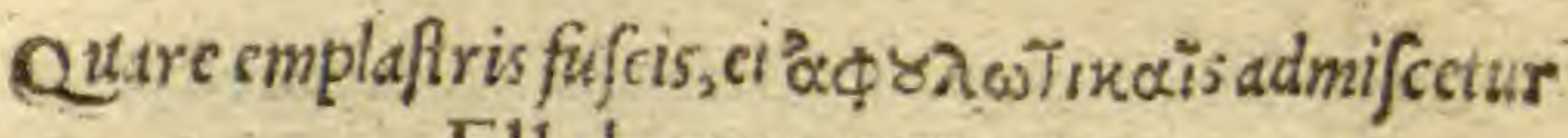
Elleborus uerarum.

Holleborus, uter $q$; calcfact, of ficcat in tertiadi $=$ Pantia, unde fit, ut abltergenis 0 acris fint potcntie, Ad leprofa quág'; comnodt funt. Niger etlam fiftulis tribus diebus affufus, callum detrahit.

\section{Elichryfon,}

Helichry] on, funt quichrifanton, fuat etiă qui amas ranthum uocent Coronaria planta, comam habet, dus reum colorem oftendentem, Si quis cum uino biberit, urine anguftijs, ferpentium morfibus, coxendicis dolo ribus, or ruptis opitulatur. Menfes eadem trabit, cons

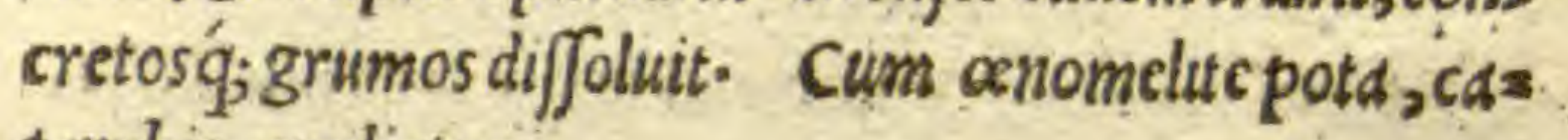
tarrbis auxiliatur.

\section{Elxine.}

Elxine, dij Perdictum nomindnt, alij Parthenium, alij Sideritim, funt qui Heracleam etiä appellutent. Vis cı tneft abftergendl, $\mathcal{O}$ fenfim conftringendi, cum bus, midttate fuffrigida.

\section{Elxinæ.}

Elxine, ca que alio nomine Cifjampelos, digerendi., facultatemobtinet.

\section{Elimus Panicum.}

Elimus, que o Meline, Mulio fimlis, ficcatorie o refrigerantis potentia, fiqudem foris emplaftretur - Si ftu quog; ueniris fluxiones.

\section{Empetron.}




\section{P. A L. AE G INETA}

Empetron, que o Praßsoides, catharticumeft phar macum, bilemac pituitam educens, Guftui falfum eft, propter quod o abfergit.

\section{Epithymum.}

Epy bymum, cxiccat ac calefacit in tertio gradu, Thymoad ominia efficacius.

Epimydion.

Eptmedij uis eft moderaterefrigcratoria, cŭ dqued bumidutate. Que fanè cataplafmate fuper impof ita, mammas turgentes cuftodit. Atunt quoq; potam ftert $=$ litatem parere.

\section{Epimelis.}

Epimclis, que o V nedon ab Italie agricolis infcrie bitur. Eft autem agreftis pomus, cuivis fructus accrbus quidemeft, nibilominus tamen ftomacho ingratus, $\mathfrak{F}^{\circ}$ capitis dolores ciens.

\section{Epipactis.}

Epipactis, que aliâs Elleborine uocatur, contra ue= nend $\mathcal{O}$ secinoris uicia bibitur.

\section{Erebinthus.}

Erebinthus, familare legumen, flatuofum eft, copio fi nutrimèti, aluo mouende, $\sigma$ ciende urime atq; lacil,

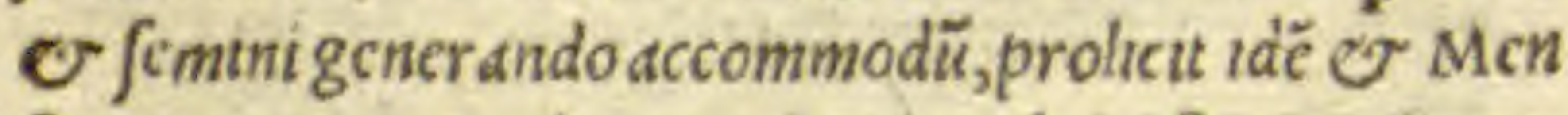
fes. Ceterum, quod uoca ur Crium hoc ift, A rietisam, h ius decoftum calculos renü confring $t$, et maxime nts gra, que Orobca uocant, his omnibuscalidiora, et $\int b a$ mura. Agr ftes tamé ad oida domefticts funt efficatiores. 


\section{DE SIM PLICIBVS. \\ Lan $x$ fordid $x$, uel fuccid $x$.}

Lane fordide, uniles funt ad irrigationcs . Siquidem

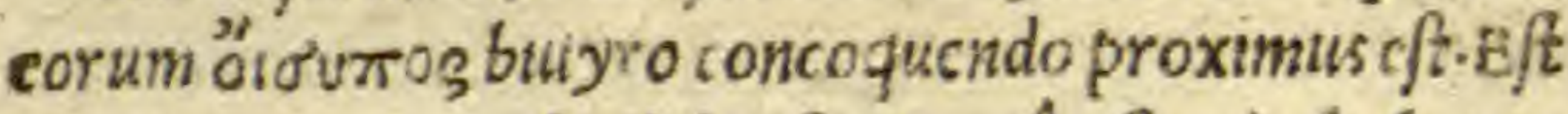
quedan, que non fordida eft - Porrô tifta uim babet $d=$

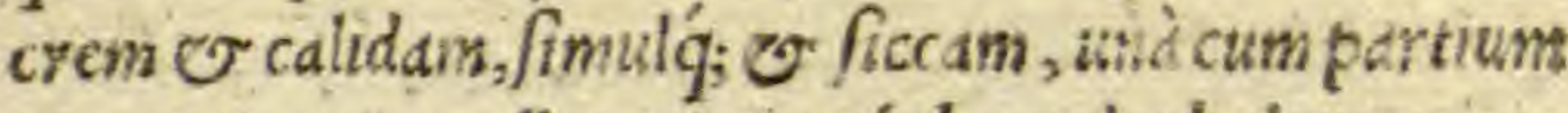
tcnuatate, que molles, nimitumq́, bumados huicerum caj vescelerrine fanat.

\section{Erice.}

Erice, fine mordicatione digerendiuim habet, ciuf of tum flore, tum folijs utilicebit.

\section{Erynos, Ephrydros.}

Erynos Ephrydros,cft hcrba, cuius fructus pondcre dracbmarum diuarum, cu mellis drachmis 4. ilttus, ocus lorum fluxü fiftut. Succus uero auriü doloribus confert

\section{Hermodactylus.}

Hermodallyli radix, cathartice ef t potentie, tü per feip $\int a m$, tum decocium eius recte $\sigma$ artheticis, tempo $=$ re quo bumores deftillant, datur. Sed fomacho admo $=$ dumaduer $\int$.

\section{Herpillum.}

- Herpillos, cale fact, adeo ut $\sigma$ urinam $\mathcal{O}$ Menfes moueat.

\section{Erythrodanum.}

Eritbrodanum, tinctorürubed, radix eft, tam acere ba $\odot$ amara, ut lienem, bcpar, o renes repurget, pre tcred $\mathcal{O}$ fanguinem mcire faciàt, Menfes $q_{\text {; }}$ mouedt, $\sigma^{\circ}$ que cuti harent, fordes exicrgat. 


\section{P A V L. AE G I NET A}

\section{Eryfimon.}

Eryfimi femen, potentiaigneum eft, $\sigma$ excalefuci $=$ ens, nusfurcio equale. Quxspropter tof tum in fermen= to, thoracem purgat, $\odot$ ineclegmatisduricias cmollit, o clancularios canceres medicatur, cataplafinate im $\Rightarrow$ poftum.

\section{Euzomon.}

Euzomon, haud dubium prioripotentia fimilitered Ic facit, sed flituof fus eff, proinde ad concubttum prori = tat. Semen urinas cit, Sed tamen agreffe fatiuo uiribus: longe prapollet,

\section{Eupatorium.}

Eupatorium, tenuium partium, \& fecantis poten: tie eft, fine calore maniffeto. Inde iecoris purgat opildes tiones, quandam ctiam participat acerbitatem.

\section{Euphorbium.}

Euphorbium, ardcntis eft potentie, $\odot$ tenuium par, tium, , imile alijs fuccis.

\section{Ephemeron.}

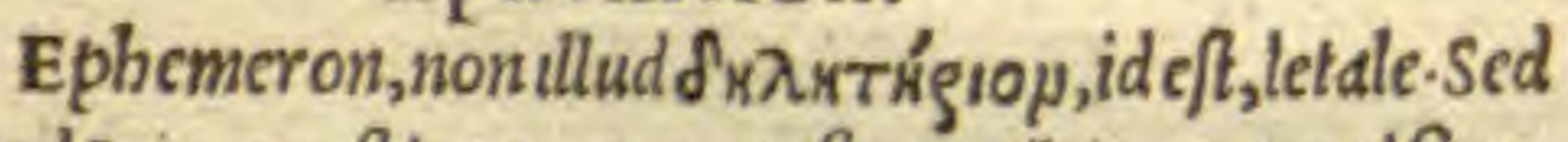
quod Iris agreflis uocatur, mifte potëtie, ctrepulfüre. Vipera.

Viperecarnes, calide \& ficce funt uehementer, per totum corpus cutem purgantes. Vnde multumeles phantia correpti, edentes $\widetilde{O}$ bibentes ip fas, fanatifunt : Que uero in madritimis \& allijs ficcis locis wictitant, ficd ciores magis funt. 


\section{Echinos.}

Echinus, herba, acerbe, explodendif;;, $\sigma$ drefaiccrs dif facultatis eft.

\section{Echinus terreftris.}

Echini terreftris fel, capillos defluentes figit. Inuete rata eiufdem caro fortiter digerit, ficcat $\sigma_{5}$ '. Potaigitur elcphantias $\mathcal{O}$ cachexias fanat. Cutis ufia, tum valent $=$ us ficcat, tum digerit quoq; magis. Alopecias igitur cu piceliquida inuncta, fanat.

\section{Echinus marinus,}

Quod de marino Echino efuiaptum eft, wrinamcit, or bonam fucit aluum. Cuius quoq; tcftafta, fimilem terreftri uirtutcm babet.

\section{Echion:}

Sunt, qui Dortadd, \& qui Alcibiadion uocent, non folum ferpentiummorfibus, cum uino pota, fuccurrit, uerumetiam prepota, mor fus eorum arcet.

\section{Hepfema.}

Muftum decoctum, quod dlij Syraon appetlant, cd= let mediocriter, $\sigma$ concoquit. Emplaficamigitur hd= bet uim, fine mordicalione.

DE HIS, QVAE INCIPI= unt ab literd $Z$.

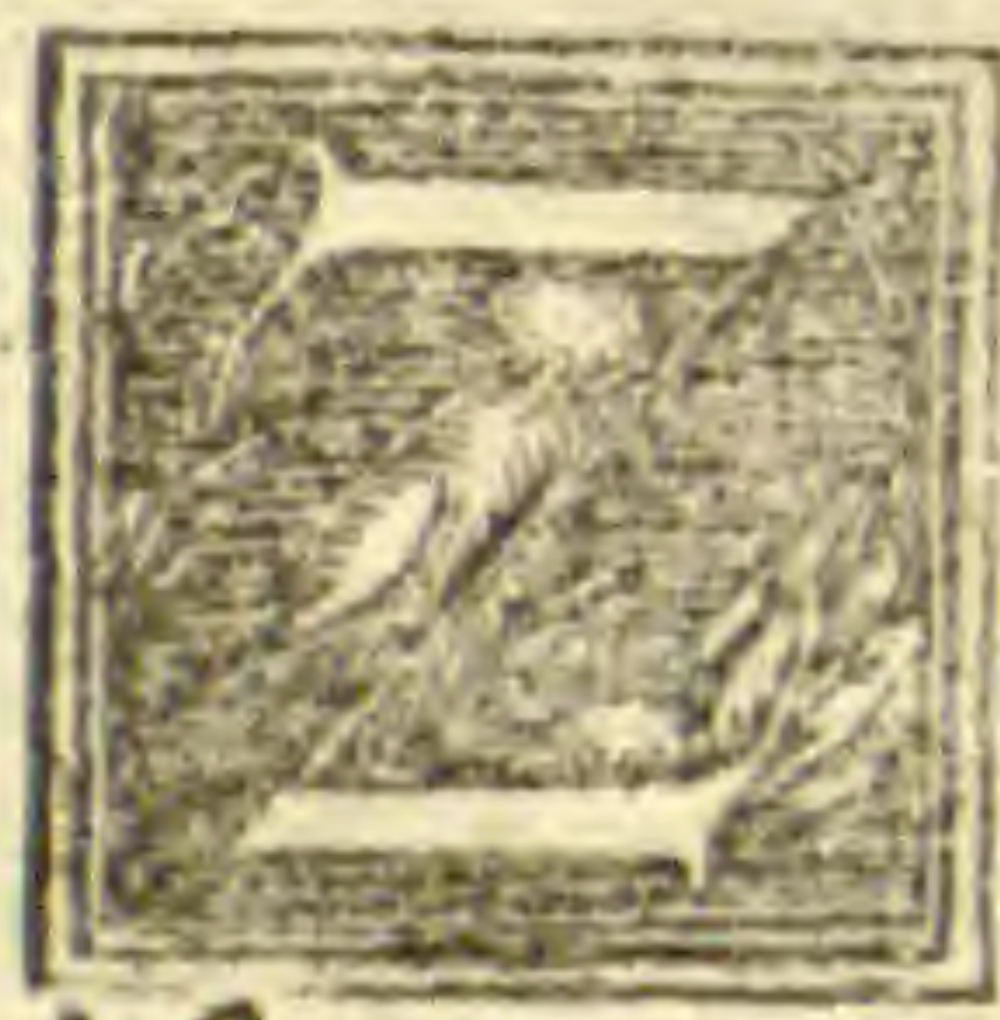

Zeia.

Eia, fimitem babet tritico facultatem, quodammodo in medio inter calefäcien deficedt, er cmplafticacft.

dum o refirigerandum, Senfin quo q; Zungiberis 


\section{P A V L. AE GIN E T A}

\section{Zingiber.}

Zingiberis radix, ualide calefacit, fed non primofta timoccur fu- Illaboratam, ov craßsiorem participat bu $=$ miditaté, propter quod perforatur facullime, E firmä conferuat caliditatcm.

\section{Zithus.}

Zithus, compofite eft fubftantie, quippe acrior eff, uipotequex putredine proueniat, frigidus ratione aco ris, undemali fucciiudicatur.

\section{Zyme, fermentum.}

Fermentum, eipfumex contrarijs polentijs confi

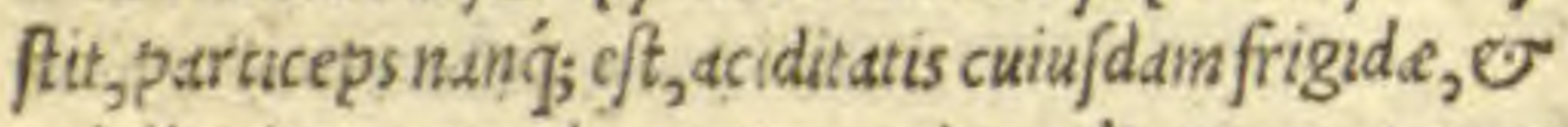
calditatis ex putredine nata, cuisccedit o ed, que ix fale est $\odot$ farina. Itaq́; mediocrier, tum calet , tum digerit.

\section{Zomos, pifcium iura.}

Ius reccntium pifcium, uentrem fubduct, per fe pus viter, E cum uino potum, 0 proprie ex phycidibus, fcorpionibus, iuldid bus, percis, $\mathcal{V}$ faxatlibus aliys tene ris, fintplicicum aquike, additis infuper anetho, ${ }_{2}$ alc, $\mathcal{O}$ oleo decofils. Simliter corum que teftaceam curems Bisbeni Tellinarum, Hiatularum, Conchularum, e tes teris Galli, cum fale multo cocti, iufcula, ciendi uirtute

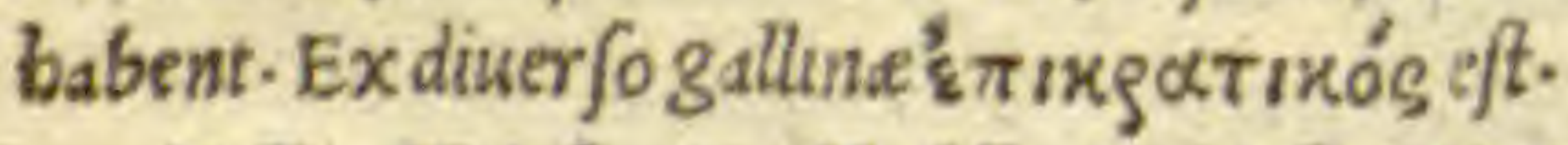

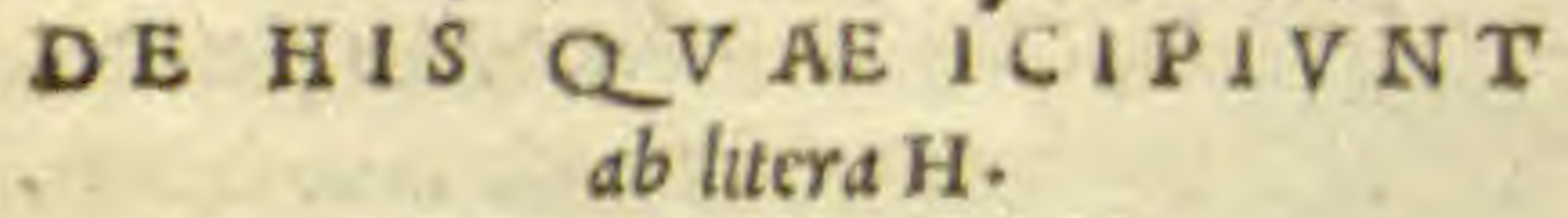

\section{Menta odorata,}




\section{DE S I M P I ICI B V S. 24}

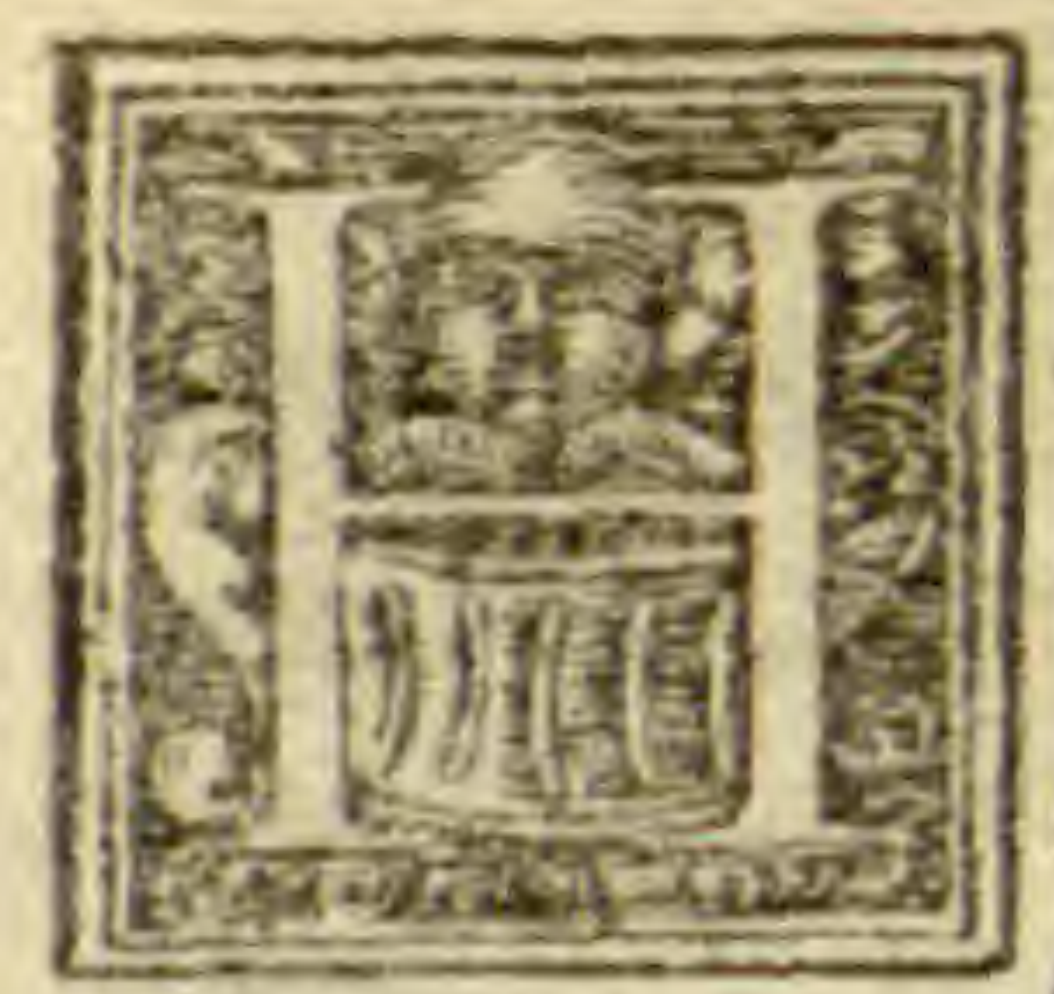

Edio fmon, callidim or tenuium partiü, scris potentie, of ex icrio feréordt ne, cum quaddan bumidta e femicoct $d$, propicr quim in Vencrem excitat $\cdot \mathbf{H} \boldsymbol{u}$ bet $\mathcal{O}$ quiddam amarum, o illo quide tumbricos interfict - Ratione accrbuatis uero, fi cum oxycrato bıbatur, recěies fanguinis ret: Ẽ öes reprimut Hadyfaron.

Hedifarum, aut pelecynus, amarum of fubaccrbum eft, quamobrem potum, gratum eft ftomacho, ov uifces rumobftructiones expurgat.

\section{Heliotropium.}

Heliotropium maius, quod o fcorpiuron uocatur. calide $\mathcal{O}$ ficce, at $q_{;}$abftergentis eft potentie. Cuius de coctum potum, phlegma ducit o choleram. Fructus ue ro cius cataplafmate impofitus, myrmetias, of fique funt alia cius generis, deficcat.

\section{Heliotropium minus.}

Minus Heliotropium, eddem poteft. Proindecŭ ni= tro, by ffopo, o cardamomo, uentrislumbricos pellit.

\section{Electrum.}

Electrum, Populi arboris lachryma, que ex ea des fillans, congela cit colore auri. Tritum, potum $q_{j}, f_{0}=$ machio $\mathcal{O}$ alut fluores, $\mathcal{O}$ fanguinem fiftit.

\section{Hemerocallis.}

Hemerocallis, raairx fomlem llio facultatem habet, prodeft'q, non fccus, atq; hilum,'gnambuftis.

Hemionitis 


\section{PAV L. AE G I NETA}

\section{Hemionitis.}

Hem onitis, adftringende uires cum amaritudinch bet, proptcr quod in acceo dd minuendas lencs bibtur.

\section{Hepar.}

Hepar rabiofic anis toftum atgicomeftum, àcanera biensi morfis auceliari creditur.

\section{Hepar Capra.}

Hcputiscapre fanies, infellat oculis luf ciofis, quos Grociucant vurTó $\lambda \omega \pi \alpha$, iulat. Et decoctt uaporé fi quis accopert, f twiliteroc ulis prodeft. Tradüt quog; caprini iecinoris sibo, comitiales, imprimis deprchendi:

\section{Hepar Capri.}

Hepar caprinum, ad eadem ficit, ad que or capre.

\section{Hepar Lacerta.}

Ldcerte bipar, denibus putridis at q; arrofis impo ftum, indolenti: mlargitur.

\section{Hepar Lupi.}

Hepar lupi, unàcum Eupatorio pracipuum phars macum hepaticum effictur.

\section{Hepar Afini.}

Iecur a ini, comitialibus incibo toftum, prodeft.

\section{Iecur Apri.}

Tecuraprirecens, fi ar faicumteratur, ex uino pos tum, contra fer pentum, $u$ lucrumí; morfus, auxilio efto

$$
\text { HeparMergi. }
$$

Mergiiecur. ficcum potum, caiculos exirudit.

\section{Erigeron.}


DESIMPÉCIBVS.

Erigeron, mixtam habet potentiam, refrigeratori= am uidelicet, o mediocriter digerentem.

\section{Eringium.}

Erinigitum, calefacit quidem, fed nonmanifftario. Exiccat uero hatud fegniter.

\section{E H I IS Q $V A E$ I Na cipiunt ḋ literd $\theta$. Aqua Maris.}

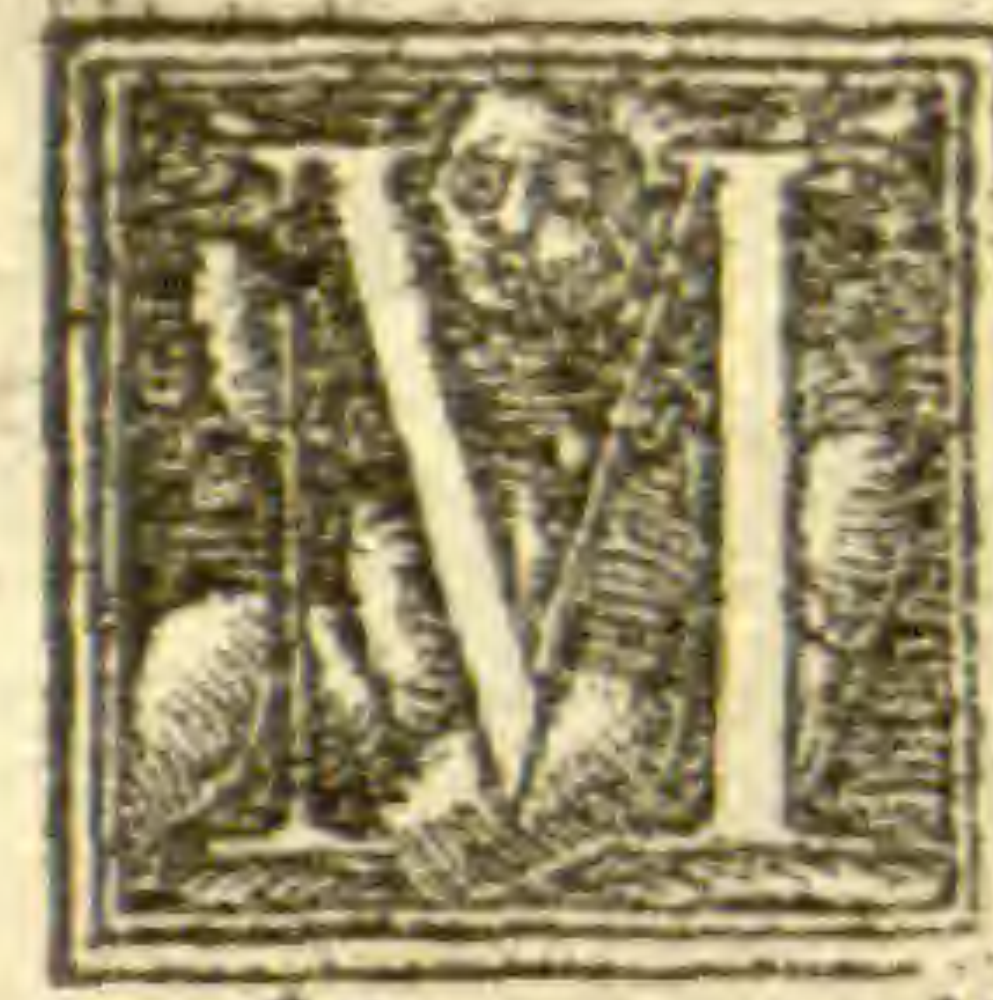

Arina aqua, adftringit e calefacit, me dio modo, er pota, ftomacho eft inimi= ca. Nam uentrem conturbat, er pitui= tam educit. Calidatamen exhaufta, at= trabit atque digcrit.

\section{Thalietrum.}

vim babet ficcam, /ine morfu-Itaq; , hulcera inuete= rats ad cicatricem perducit.

\section{Thapfia.}

Thapfia, acrem acualenter calefacientem facultas temcum humiditate coniunetam habet - Qutamobrems ex alto uiolenter extrabit, extraciumq; digerit.

\section{Sulphur.}

Sulphur, retrahediuim poßidet, eft q́; temperamen ticalidisatg; efentix tenuis, adco ut ad multa uenenato rum antmalium refiftat, et maxime aduer fus tusturem marinum, o draconcm . Pratcrea ficcuminfperfum, uel mixtü fakiua, aut ccrte pusriurina uel oleo, aut ax=

E ungis 
$=$ PAVL. AEGINETA

inguia porci,fue melle, $p$ for as mirabilter fanat.

\section{Lupinus.}

Thermus, id eft, lupinus, ille qui e fui idoneus $c f t, c m$ plaftica of potentie, amarus difcutiensq;; of ficcus, $a b \int_{q}$; mordicatione.Vermes perimit, tam catdplafmate illttus, quãetiam potus- Quin $\odot$ interancd, atq; intefit na repurgat, cum ruta $\odot$ pipere decoctus potusq. ${ }^{\circ} \mathrm{C} u$ tem etiamemundat, $\sigma$ tubera difcutit, cum oxymeille, ad inftar Cataplafmatis illtus.

\section{Lupinus Agreftis.}

Agrefis lupinus amarior cft, $\odot$ ad omnia domefti coulentior.

\section{Thelipteris.}

Thelipterin, alij Nympheam, uel pterin appellităt.

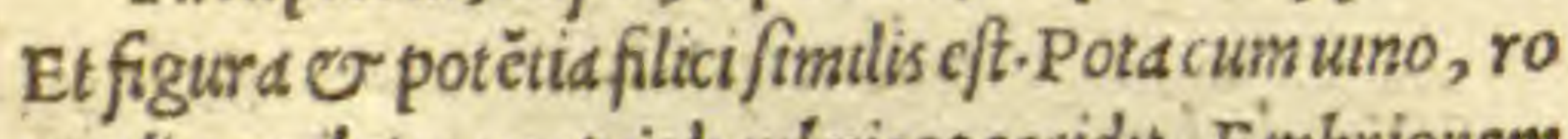
tundos $\sigma$ latos uentris lumbricosoccidet, Embrionem in uterocorrumpit, $\mathcal{O}$ abortere facit. Foliaciuscom= mefta, uentrem moliunt. :

\section{Thlafpis.}

Thlafpi, femen eft, $\sigma^{2}$ ip fum acre babet quiddam, adco ut internos abfceffus potü dılrumpat, Menfeseis et $\odot$ foetum necat . Per fedem tinfufum fangunolenta

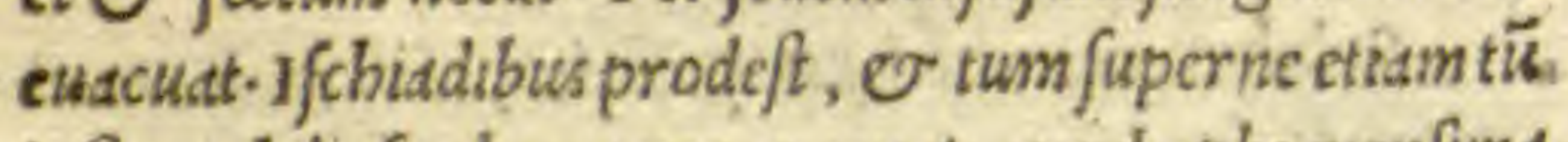
inferne, bullofos humores euacuat, oxybaphs menfurd pota.

\section{Thridaxlactuca.}

Laclucd, frigidü O bumidü bolus, nó utiq, extremo modo, fed ut fontana aqua p frigida. Idco aduer fus phlcs gmones caldas congruit . Poid fitum etlum extingutt, \& feminis 
- Jeminis fuxus cohibet, nec non $\mathcal{F}$ Veneris in fomno umaginationes.

\section{Lactuca agreftis.}

Agrefic lactuca, bac minor o ipfa refrigerdt 0 bumectat, fed fucculcutior oft.

\section{Thymus.}

Thymus, calefacit $\mathcal{O}$ ficcat in tertio ordinc, lubri $=$

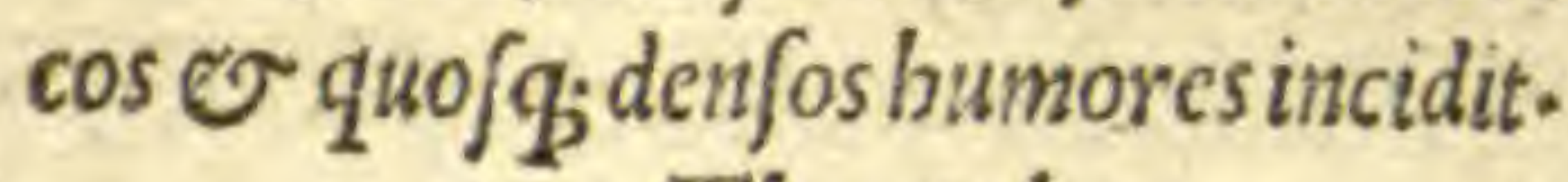

$$
\text { Thymbra. }
$$

Thymira, prefertim agreftis, proximan Thymo uimhabet-Hortê $f i s$, etiäfi uiribus imbecillior, ad opfo mia tamenmelior.

\section{Thymelęa.} fatem habet.

\section{DE HIIS Q $V$ AE I $N=$} cupiunt à litera $\mathrm{I}$.

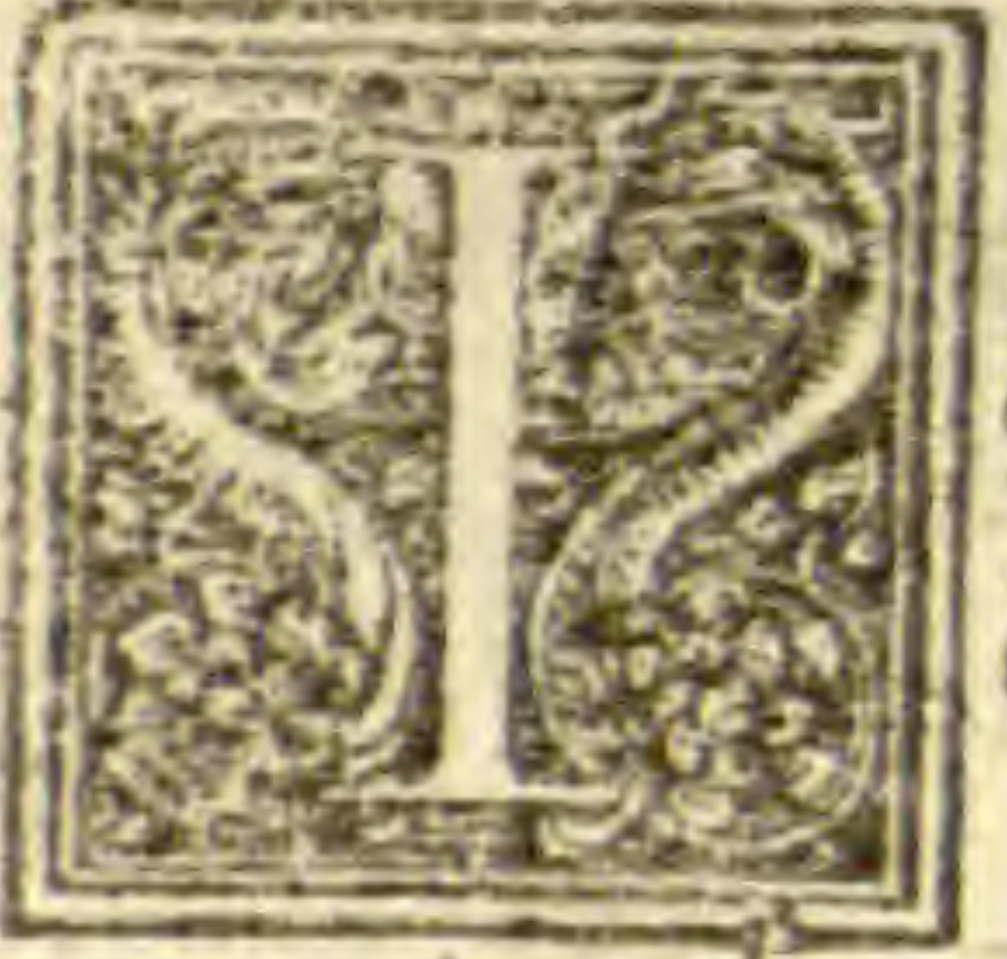

Dearadix, o guftue potentiadcer ba, fanguinis proflumia o fluxus, tum pota, tum foris impofita, curat.

\section{Sudor.}

Sudor, $/ 2$ c cp ratim quidè coligatur, efficax cft, ni fceiur aũt in paleftris fefe excrcitantiü uie babenti, o fit totüdıgcrë is potẽtie, nutricibus ardëtrbiis prefécns eft remedu, $\odot$ ad extinguëdü quod in ip $/$ is ardet. Pre fidio eft 0 aduerfus Bubones-Sisero ficcus fuerit, hu mectatur cuim Caprinotel rofeooleo.

$$
\text { Ixosuifcum. E } 2 \text { Ixos: }
$$




\section{P A $\vee$ L. AE G I N E T A}

Ixos, calefacit cum quadam acrimonia. Itaq́; ualens ter ex profundobumores extrabit, cas q́; digerit, $\mathrm{Td}=$

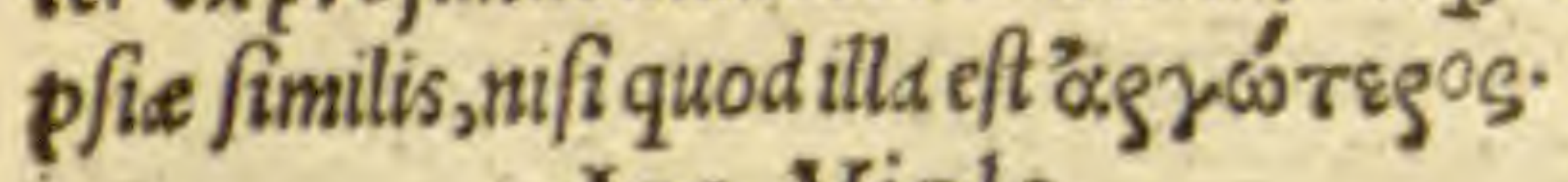

\section{Ios, Viola.}

viole folid, aquofamatq; fubfrigidam fubftantidms fuperantem poßident.

\section{Ios, Aerugo.}

Acrugo guftantibus acrem qualitatem offert diges rerens, detrahens, $\sigma$ liquans, non tencram modo, fed o duram carnem. Quod fi palculum eius, cerato non pausoadmifceas, id quod exambolus miftume ft, citra morfumexterget.

\section{Hippocampas.}

Hippocampus, marnü eft anumal. Eius cremati ci= nis cumliquidapice, alopecias replet.

Hippolapathon, Granctis rumex.

Hippolapathon, nafcitur in paluftribus, habet'f eafdem cum lapathourires.

\section{Hippomarathron.}

Hippomarathron, Canchri fimile, pauloficcioris potentie quàm fit marathrum, propter quod aluum $f i$ Ptit. Calculos atterit o $\mathrm{M}$ Mnfestrahit.

\section{Dealio Hippomarathro.}

Aliud Hippomarathrü femé habet Coriādro finile? facultate quidè hippomaratbro fimile, fed imbecilluss.

Hippopotamus.

Hippopotanus, td eff, cquu funiatilistrficulus exica cattus, 


\section{DE SIM PLICIBVS.}

catus, ¿c potus, cötrd ferpentiü mor fus utiliter bibitur. Hippofelinon.

Hippofclinon, uulgato felino facultatiadmodum $f_{i=}$ mile, preterqquam quod lllo debilus eft.

\section{Hippuris.}

Hippuris, adf tringentem cum amaritudine faculta= tem poßidet, ac protide ualcnter, fimul'́; ; citrd mordi= cationem exicceat. Vulnera igitur maximacöglutinat,

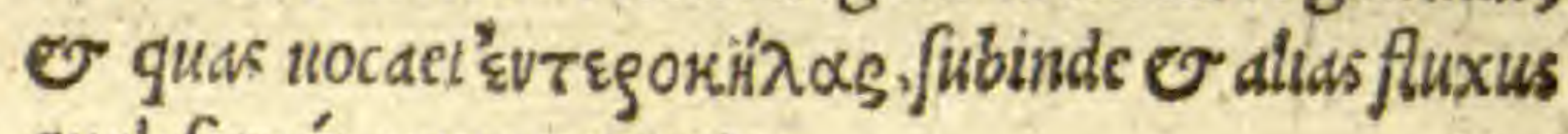
quale fcun'́; cum umo pota.

\section{Hippophaes.}

Hippophaes, ab aijis heppophaos, quo ueftes uellia cando poliunt-Surculo fus frutex $\mathrm{e} f \mathrm{t}$, é cuius radice fuc= cus expromitur, uelutı é Tapfla, qui obolipondere da= tus, cü erui fartna, purgat Cholerä o phlegma. Quin $\mathcal{O}$ tota plantula ficcata Cartharticam uim retinet.

\section{Iris.}

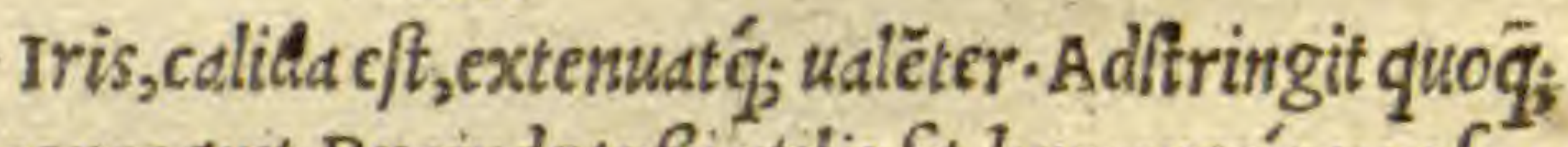
ac concoquit.-Proinde tußi utilis fit, humores $\sigma_{;}$non $\mathrm{fa}_{\mathrm{a}}=$ cile excreabiles extenuat - Torminubus medetur, $\mathcal{O}$ for didahulcera emundat. Pota cü melicrato, uentre č ducit.

\section{Ifatis.}

Ifatis domeftca, qua tinclores utütur, facultatis eft exiccantis. Habens quiddã etiă ad/tringens. I deo ó; $m a=$ gna hulccra, durorü corporü glutinat. Et fangumis $p=$ fluuio laboranttb. auxiliatur-Tumores oedematodeis digerit $\mathrm{O}$ f fiftit etiä cfficaciter cunctis hulccribus $m a=$ liginis, que Cacocthe Hocantur. 


\section{PA $D$ L. AEG INETA \\ Iris fylueftris.}

Iris fylueftris, preterquä quod magis mordicat, etiä ficcioris eff potentle, potentißime ctiam putredinbus. obfiftit. Ad cateraucrodeterior. Alloqui propter facul tatis robur, lienofis quog; utilis.

\section{Ifopyron, aut Phafiolon.}

1fopyron, uel phafiolon, femen amarum o fubdcer bumbabet, Extergit itaq; 0 incidit bumores craffos, cühoc ut corporacōtrahat atg; cöftringat Purgat etia ex alto. Et fanguinem [puentibus nonc/t dduerjum.

\section{Itea; Salix.}

Salicis, tum folia tum flores, fine acredine ficca funt potentie. Ettam/i modicc adftrmgant, Cortex ficctor, Cutusufticinis, ex bijs cft , quecum accto tminodice de ficcant-Clauos 0 myrmetias, of quicquid eft eius ges neris. Ichthyocolla.

Glutem pifcium, Emplaffice o f fucer oft facultatis.

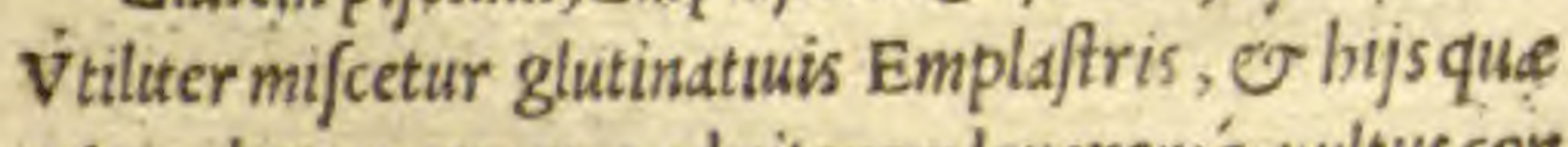
cointra lepras, e que ad nitorcmlcuoremíg uultuscon ficuntur.

DE HIIS Q V AE I N= ciptunt àltererd $x$.

\section{Cancanus.}

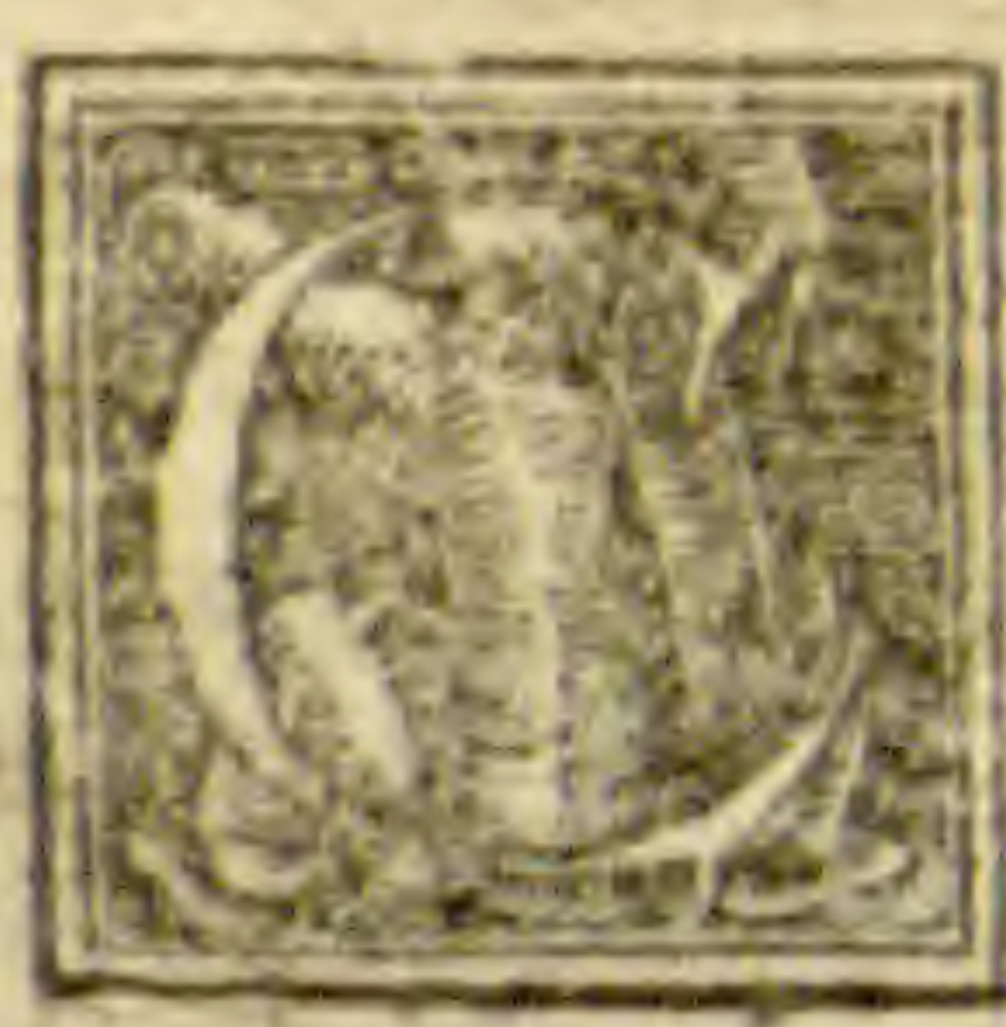
Ancam radix ficcacft mediocriter, $\mathcal{V}$ finc mordicatiöe Emplaftcca. Qutupro pter afperäarteriäun eclegmatc, $\sigma$ cü unno liuat, aut fubacta etlams : Qucm= admodum tragacantha, uel glycyrizd. 


\section{DESIMPIICIBVS。}

\section{Canchamum.}

Canchamum, lachryma c/t in Arabia na fcétis drbo ris, myrr b e fimlitudine, et uirofo in guftu odore.V fus eius ad Thymiamata, ideft, fuccenfioné odor $\tilde{h}$, ov ud = pordtiones, Facultatem habet, pinguia quéq; macerans diabftergendíg;, $\odot$ abobftructioneliberandi.

\section{Cadmix.}

Cadmie ambe cxiccant. Atcraßiorŭ partium eft, que uocatur ßoт guítıอ, magis tenuiü parcium $\approx \lambda \alpha$ หiтKs, vftacadmla fine mordicatione ficcat 0 deter $=$ git.Vtilis ad bulcera, carnis repletioné pofcétid, $\mathcal{V}$ in oculis or in totocorpore, maxime in corporibus bumi= dioribus molliorıbus $q$, A t in cöpactioribus, efficatiori= busopus erit.

\section{Cacalia.}

i. Cacalia, fimilěcü càncano facultatem ơ utilitatem babet.

\section{Calamagroftis.}

$\therefore$ Calamiagroftis deficcat, o proinde fuccus fue de= coctü radicü, opththalamtis facultatibus mifcetur. Se= men urinam ciet, „dco q $_{\text {; }} \mathcal{F}$ ftomadbo conucnit.

\section{Calaminthe.}

Calaminthe, calidi o ficcitěperamenticft, ex ter $\Rightarrow$ tio quodāmodoordine. Parnŭ quoq; tertuй cūacrimo= mia, multoulenttor Hedyof mo fiue pulegio, nëpe ogre ftis ip $f \mathrm{H}$ Hedyofmos. Promptiter ergo queque in pro $=$ fundo harent ad fuperfictem extrabut. Nectio radě ra tione $\mathcal{O}$ fudores prolteat - Vniucer am pretered cor $p$ is diftrahit, fibriu rigorcs, cü olcotrica, fubinde quie fce $=$ rcfacit. Et If hradicis mhil adco profentancum reme $=$ 


\section{PAVI. AE G I NET A}

diume eft . Que cuti adherët, bumores malas abfergit.

\section{Calamus aromaticus.}

Calamus aromaticus, calefacit $v$ ficcat in fecundo ordine-Habet aŭt quiddă partium tenuium feiptic ǔqu;" Vrină igitur ducit E ftomachicis, queq́;; ad hepar e fecundas faciüe facultatibus, commode mifcetur.

\section{Calamus phragmites.}

Calamus phragmites abftergit, ceterum folia uires tia mediocriterreffigerant.

\section{Calami Cortex.}

Calamidromatici cortex uftus, cale facit feré inter= tiadiftantia - Radiceme eus cu un bulbis cataplafnate im = pofitamtela, $\odot$ pinas extrabere ferunt.

\section{Campx.}

Campe, id eft que in holeribus naf cuntur eruce, $\dot{\alpha}$ uenenatorü iniurijs, que iĞu aut morfu uenenü relin quint trite, ex oleo inuncle, ferütur bomincs feruare.

\section{Cantharides.}

Cantharides, que in tritico inueniuntur, luteos 0 - bliquos zingulos habentes, feruentis acctiuapore fuf

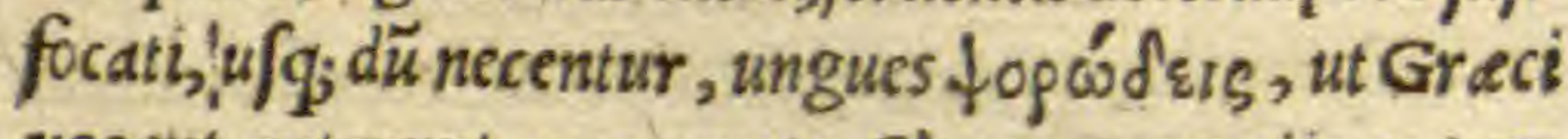
uocant, exterunt, cum cerato. Clauos quoq, eijctunt o. Icprofa queq́; medicantur. Numerantur inter medicd menta cauftica, $\sigma$ que uim habent crodendi. Quidam earum quiddam in medicamenta urinam mouentla mis fcentes, minuftrauerunt.

\section{Cannabis.}

Comnabis fructus, flatus extinguit adeoǵf, deficcat, ut genitu $=$ 
DESIM PIICIBVS. 29 genituramexiccet. Verum fuccus uiridis ad aturitum do lores, que uidelicet exobftructione funt, facit.

\section{Canopon.}

Canopon, Atte floseft, de quo in fuperioribus di= Etumeft.

\section{Capnos, Fumus.}

Capnion, uel Capnos, fic inde adpellata herba, quia fuccus eius, non paululumlachrymas trabit. Oculorum aciem exacuit, acris fimul o amare qualitatis. Ad hec urinas quog; multabiliofa cducit, medetur'q; lecoris opi Iationibus $\odot$ languoribus - Stomachum uuat, $\odot$ uen * trom fubducit.

\section{Capparis.}

Capparisradla, ab/tergit, purgat, incidit, 0 difcus tit cum quadam amaritudine, fimul $\sigma$ acrinonia.Por= rô etiam contrahere, denjarc, conftringere quoq;ido= nea, cum quadam acerbitate, propter quod lienes und $u=$ ratosiunat pota. Et foris impo fita, Menfes mouet, $\odot$ excapite purgat, detrabit $q_{;}$, ac hulcerbus ettam accom moda, quecacoethe appellantur, ordentiumdolores mi tigat. Tumores difcuttt, שV uitiligines. Subinde etiam auribus uermes exirabit. Folia $\mathcal{O}$ fructus f tmilem wim obtunét, imbccillioris tamen potentia.

\section{Nafturcium.}

Cardami femen, adurentis nature eft, uelut findpi, proinde rubificat catapla fmate impo fitü. Crafjos quo\%s humores cüalijs potum, $\sqrt{a}$ and - Herba ipfa propè ciuf $=$

$$
\text { E } 5 \text { demeft }
$$




\section{PA V L. AE G INET A}

demeft facultatis cum femine. Verumbumida ef medis ocriter, $\mathcal{O}$ crudd in obfonijs eftur.

\section{Cardamomum.}

Cardamomum, acre quidem $\sigma$ boc, fed Nafurcio multo imbecillus. Habet quiddăctiä amaritudwns, pro pter quod uermes necat, $\odot$ pforas cü aceto abftergit.

\section{Caris.}

- Caris refoluta, cü bryonie radice pota, ucrmes occidtt. Cancrifluuiales.

Cancrorum fluuiallüuftorum cinus exiccatorius eft, fimilis cineri Echinorum, $\mathcal{E}$ Cochlearum, à propricta tetamen fubftantie fua morabiliseft un his, qui à canęr biofo funt demor $f_{i}, f i$ in potuexhibeatur.

Cancri marini.

- Cancrimarini, multis numeris prioribus funt ficcie Yes, e- non nifi ualenter ficcare uolentibus, uf wi funt: Fiuvialls Cancer tritus, $\mathcal{O}$ cataplafmatus, cufpides $\mathcal{E}$ : folopas extrabit.

\section{Caron.}

Carifemen calc facit $\mathcal{E}$ exiccat in tertio fer é ordine: Flatus extingit, $\sigma$ urinas cit . Eadem preftat $\odot$ tota planta.

\section{Carpefium.}

Carpe fum, aromaticumeft, o tenuium partium, abfergutigitur vifcerum obftructiones, o urinas cit. Renes quof; ca'culis pregrauatos expurgat.

\section{Caria, Nuçes.}


Arbor tum in folijs, tum in germinibus ddftrictio= nem qquandam poßtdet, maxime in putamine. Suecus ita'f; corum decoctus in melle, ftomachicum fit pharmat cum. Cortex partium tenuium eft, 0 ficcat, uerum $f=$ ne mordicatione. Porrô ipfius id quodedendoeft,olco $=$ fum eff, o fucile exprtmitur . Oleum ip fum fubtilium eft partium, $\mathcal{\sigma}$ digerens. Tumores $\mathcal{O}$ putredines pro pier uetuftatem abfergentem babent facultatem eor $\tilde{u}$, que funt circa cutem.

\section{Nux Pontica, Auellana.}

Nux Pontica, quam nucem paruan uocant, Grece $\lambda \varepsilon \pi$ Toxápvop, nature eft magis refrigerantis $\sigma$ dH= fitre. Ceicra magne nucifumilis.

\section{Caryophyllon.}

Caryophyllon, nucıfoliu, ctiamfi hanc non habet na turam, quã nomen pretendit. Sed cx India flores arbo ris cuinfdā feltucacei funt et nigri, logitudine pollicari, acres, odorati, $\mathcal{O}$ fubamari, calidi et ficci quodamodo in 3. gradu, optımü $v$ multis pharmacis cömodißumü.

\section{Caffamon.}

Caffamon, quidam ferwint effe fructum Balfami, de quo fupra in Bal amo diffume eft.

\section{Caffia.}

Caßsia cale facit o ficcat in tertio quodämodo ordi= ne, elt $\dot{q}_{\text {f }}$ fatis partiütenuium, acris, $\mathcal{O}^{\circ}$ ddftringens me diocrtter : Quamobrẽ incidu, fimul'q; diftrabit, dd hac

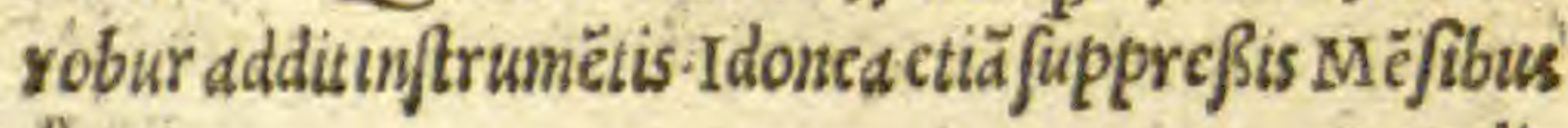




\section{P. A V L: AE G I NET A.}

\section{Caucalis.}

Catucalis, alij Daucum agreftem uocant, calefacit,

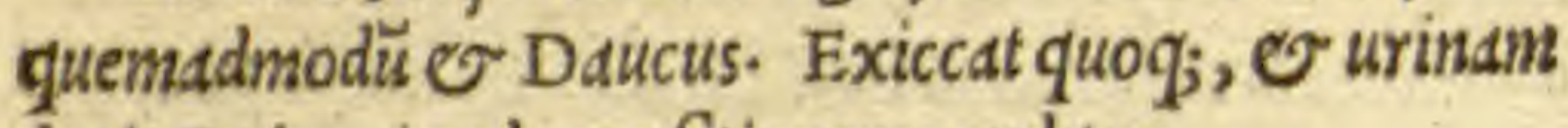
ducit. Et inuridadrepofitionem condutur.

\section{Cenchros.}

Miliumrefrigerat quidem in primo ordine, exiccat ueroinfecundo, paululum etiam habet tenuitatis. Des foris igitur in facculis calefactum, $\mathcal{O}$ caldaplafmatum, exiccat.

\section{Cedrus.}

Cedrus, queddam eft frutico fa, fimilis Iunipero، Et arbor quidem caleficit $\mathcal{O}$ ficcat in tertio ordine. Cete rum quod ex ea ef toleü, quod Grece uocant xed paíap, quartumattingit ordinem, manifefte tenuum partū. Mollesitaq; carnes, promptiter, citra '; dolorem putre ficit, dur as ucro, $\mathcal{\sigma}$ mortuorum, ficcat, $\odot$ d putredine

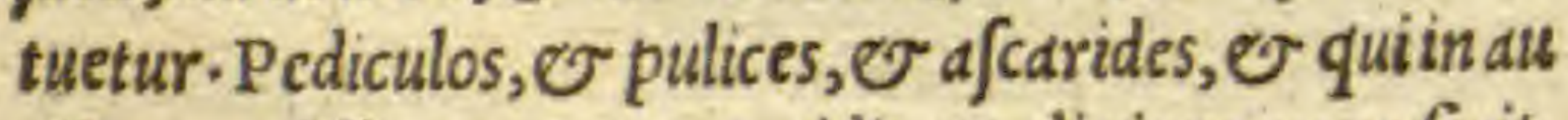
ribus enafcütur uermes, occidit, $\mathcal{O}$ aliainnumera facit .

\section{Centaureum.}

Centarcimaioris radix, acris eft, $\mathcal{\sigma}$ cale facit, cum terrofa quadam adffringentia. Menfes itaq; proulocat, of foetum mortuumenccat o euellit . Vulnera conglu tinat, $\mathcal{O}$ fanguinem eijcientibus prodeft. Edquog; it $=$ uat, que funt circa thoracem.

\section{Centaureum minus.}

Minoris Centaurci radix planè inefficax, inofficios fág. At ramiualenter, $\sigma$ fine moralicatione calc facciüt, adomina 


\section{DE SIM P LICIB $\nabla S$}

ad ommiautileis, ad que memoratü maius Centareum. Decoctum, herbe If chiadicis iniecium, bilofa quedams educit, o quidem cruentaubi eiecerit, magis iunat.

\section{Cornu Capra, \& Cornu Cerui.}

Ex Cornibus Capre o Cerui, dentifrcium fit, le* wigandis dentibus utile. Gingiuas etiam molliciefuidas contrabunt, Cerui ctiam cornu cum uino tritum, cata $=$ plafmatis $q_{;}$mftar illitum, commotos dentes figit - Por= rôab uftione clotum, dyffenteriam, coliacam paßione, fangunis putd, $\odot$ morbum aquaticum curat, pondere cochleariorum duorum, mifcetur e ophthalmicis mes dicamentis.

\section{Cornu Tauri.}

Tauricornucüaqua potü,Hemorrhagias cöpefcit. Cerafia.

Cerafia, quedam dulcia habentur, quedamaufteraDulcia citiß 3 ume dilabuntur. Auftera ftomacho vititiora funt, pituitofis $\mathcal{O}$ excremento of ftomachis consfrunt, propter incidendi uim quam habent.

\section{Gummi Ceraforum.}

Ex Cerafo arbore, quod deftelldet gummi,aperas ar teriaslenit. Vtile ctiam calculofis in uino potum.

\section{Ceratonia.}

Ceratonia, exiccantis $\mathcal{\sigma}$ adffring ent is eft facultatis ficut $\mathcal{O}$ fructus cius, que Ceratia nocant. Nonnibletia dulcedinis continens.

\section{Capita Menidum.}




\section{$P A \nabla L . A E G I N E T A$}

Capita M cenidum, fale inueteratarü ufts, ficce funts. potentie, nor admodum acris. Sedis rimas, quasuorät sáryoofoss o Columellam diu induratam, fanant.

\section{Capita Smaridum.}

Smaradiscaput uftum, alopecias fanat, cum adipe urfino.

\section{Caput Muris.}

Caput muris uftum, eadem preftat, que fmaridum capita, cummelle.

\section{Caput Milui.}

- Miluicapitulum, mutilum, ucl ficcum, finteplumis, potium cum aqua, pondere quantütribus digitulis com = prebendas, eis qui ex pedum dolore laboraint, indolen= tiam pollicetur.

\section{Caput Lacertx.}

Caput Lacerte, ferunt folopas, extrahere o myr mecias, $\mathbb{\sigma}$ acrochordonas, $\sigma$ clauos.

\section{Cecis, Galla.}

Gallh, ed que Omphacitisnuncupatur, admodüacer bumeft medicamen, unde deficcdt, $\sigma$ repercutit fluxio nes. Ad hec conffringit, contrabit'́, partes laxas er languidas, ommibus' ; fluxionü affectubusftrenuc re $f$ is Pit. Eftouerotertij in deficcando, fecundíg; in refrie gerandoordnis. Alterd dutem Galla, flauailhe magind, deficcat quidem er ipfa, fed minus priori,tan to, quanto plus acerbitatis relinquitur. Sedis phlegmo nas, o procidcntias iunat. V/fte antem, $\sigma$ in acetoexs 


\section{E SIM PLICI B VS.}

tincte, fanguinis retincndi facultatcm acquirunt.

\section{Cepea.}

Cepea, Portulace fimilis eft. Bibuntur in utno folia eius utiliter contra uefica feabtem, or urime fithicidas. Prodeft'; hijs precipue, addito afparagi, quem myr= acanthum uocant, radicumdecocto. Pota, ftrangurie opilationes aperit.

\section{Cara.}

Cera, temperda, aliorum pharmacorum materia eft, ipfatamen per fecxeorum gencre eft, qua fenfim abfergunt.Habet $\odot$ paulum quiddam digerentis, $c d=$ uderǵg potentia. .

\section{Buccina.}

- Buccinarum tefta exufta, uehementer ficca potene tie redditur, idq́; fine mor fu, hulceribus ś; malignis, que cacocthe uocantur, infpergitur. Infuper cum acelo, uel tuino, autcerte cum oxymelle dd bulcera putrefcentia că ucrit, Adhucuiusntes, fi in oleo decoguantur, oleo fubs

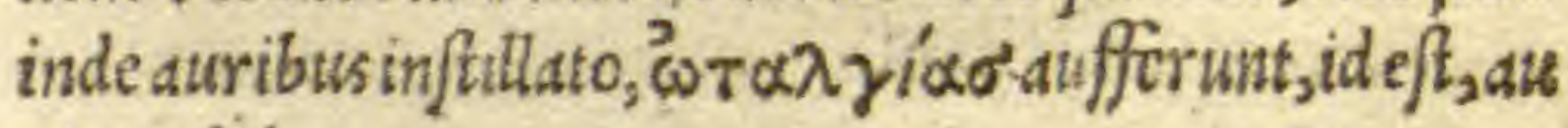
rium dolores.

\section{Cíci.}

- Recinif fructus, quemadmodum expurgat, detergit, de digcrit- Sic quoq; folum, fcd undequáq debilius, ut: in oleodictumefl.

\section{Cinnabaris.}

Cinnabaris, acris mediocriter ef potentie ; Habet $\circlearrowleft$ quiddam quo adffringit.

Cinnamomum 


\section{P A V. L: AEG IN ET A}

\section{Cinnamomum.}

Cirnamomum, omnium maxime partium eft tenui= um, non ob hoctamen fumme calidum. Nibl duté eque deficcat corum, qua pari funt excalfaciendificultate, propter tenuitatem fcilicet effentie. At Cinnamomis e/f uelut imbecillum Cinnamomum. Nörull /peudocmnn= momum appetlant.

\section{Circea.}

Circeerddix, calida eft, $v$ boniodoris, que cum ut no potd, fecundas purgat . Semen autě in forbitione ex= bibitum, dd lactis generationem conducit.

\section{Cifferis Pumex.}

Pumex, abftergentis potentue eft, o maxime den tium, id q́; adeo propter a peritatem quam habet, $V n=$ de ad pharmaca adfcifcitur hulcerum incarnatiud, $\sigma$ dd ea, que cuticulam extergunt; at q; mundificant. vfustamen,partiüredditur magis tenuium, acrior q. Ablutus ueluti mordicantisnatur a reponitur.

\section{Ciftos.}

Ciffos, uel Ciffaros, paruus frutex eft, acerbefcens, fed tepida quadam frigiditate. Ceterum, folid eius $\mathcal{O}$ ramuliin tantum de ficcant, ut uulner a etiā adglutinent. Flores ficciores in fecundo quodammodo ordine haben tur, unde dy fenterlas, ovalias fluxiones fanant poti. Deforis cataplafmatis rituillita, hulcerum putredinos fo iunat:

\section{Ciffos, Hedera,}




\section{DE SIMP L I CIB V S.}

Cifjos, excontrarijscon/tat potentijs, fringit enim - refrigerat atq; cum quadam acrimonia calefacit, efentia aquo $\int a$ particeps, uiridis tepids. Viridis ergo foliaincocta uino, tulnera conglutinant.Ambuftaigne, of Pleneticosiuuant - Succus medicamentumeft' $\rho_{\rho} \rho_{i}=$ vop, id eft, quod naribusinfufum caput purgat, tumue tuff tas aurrü fluxiones fanat. Lachryma eus longe acu= tior, lendes interimit o piles nudat.

\section{Citrium,}

Citrium, quod $\sigma$ medicum malum nocdnt, quod in

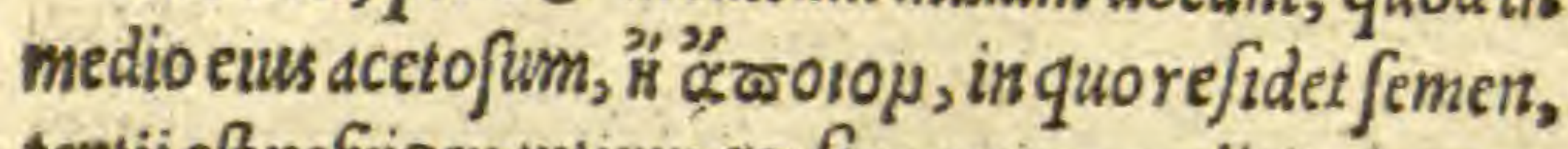
tertijeft refrigerantium $\odot$ ficcantiumordinis. Cortex ficcat quidem, insecunda duftantia, refrigerut ucronă adeo, alioquiacris eft. Carodenfioris fucci, humtda eft, refrigerans, femen exedit, $v$ exiccat in fecundo ord $l=$ ne. Et folia arboris ficca, dif cuttientis funt facultatis.

\section{Chichorium.}

Chichorium, uel picris, agreftis seris eft, refrigerat $\checkmark$ deficcat in fecüdo ordine.Habet quiddam etuam ad =

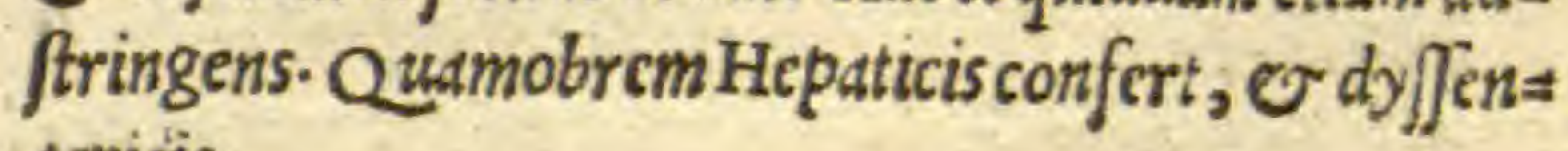
tericis.

\section{Clematitis.}

Clematitis, que ab qubufdam uocatur, Daphnoides abalïs Myrfinoides, ànonnullis Polygonoides, viticu= lam babet prelongam, uelut olof choeni, craffa, folto laus

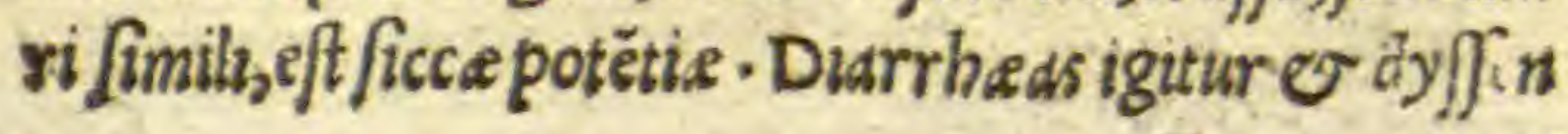




\section{PAVL. AE G I N E T A}

terias $v$ dentium dolores fedat, 0 venchatorum nors fibus anxuliatur.

\section{Clematiris altera.}

Clematitis altera, circumuoluit $\int e, f\left(1 n d i{ }^{\prime} q_{;}\right.$arbores ut fimilax, acute o $\sigma$ caufica faculians. Tritum femen etus in aqua potü, per in ferna bulem $\sigma$ p ptuitam trabut. Pelluni lepras folia cmplaftrimodo impofita.

\section{Clinopodion.}

Clinopodion, calldecf potentic, er exiccantis, fed nondumadurendi, in teruo fortapis ordune.

\section{Cnecus.}

Creci femine, duritaxat ad deicEtiones utinnor. Sicui deforis co uti hbuerit, in tertio eril calefacientü̈ordinte-

\section{Conchylia.}

Conchylia, buectnis confimidem facuitatcm habent.

\section{Ventermergi.}

Ventrem mergi, ftuc recens coctü, fue ficcatŭk susó

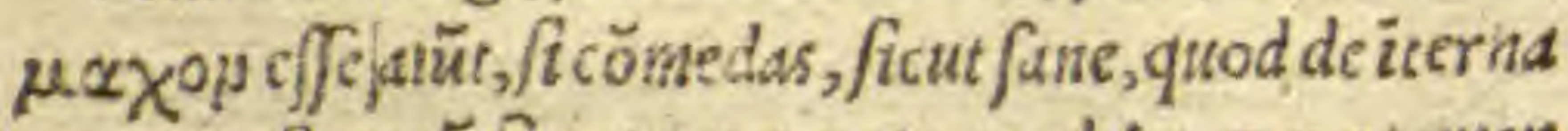
tunica gallusarü jiccata at $q_{\text {; }}$ pota, tradiont O we tamen ambo Galenus pertentans, f $x^{\prime}$ fa cffe deprehendit-Alio= quitamen palumbi auis uentrem modice potum, perlo= tum lapillos exigere, Diof corrides eft autor.

\section{Venter Muftellæ.}

Muftelle uenter in potuexhibitus, Alcxipharmas cumeffecredititi, coruin qü à uenend'is beftijs fusc de morfi. 


\section{DE S I M P L I C I B
Coccum Gnidion.}

Coccum Gridion, femen ef T Thymelee non Chames les, ut quidam falfó exiftimauerunt. Acuta potétie o adurentis. Purgat per inferna cum aqua potum.

\section{Coccus Baphicus, grainum tinctorium.}

Coccus Baphicus, fine mordicationcextecat. Proin

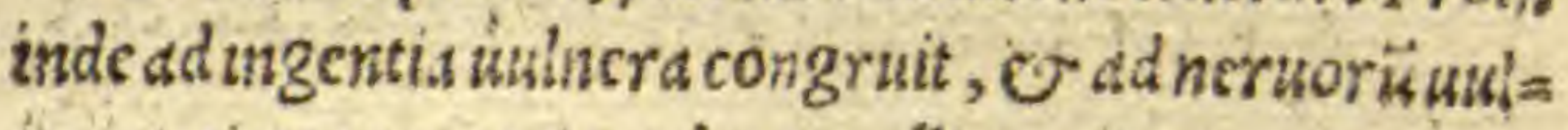
ucrat tonescum acetond oxymellae tritus.

\section{Coccimelea, Prunus.}

Coccimelce femen, uentrem fubducit, recens qui= dem plus, ficcumminus. Caterum cxeadecoctumat fubinde gargarizatü, gurgulionis uel columelle pble= gmonas fanal .Arboris gummi inccaicntis o partium tenuium potentia eft, $\circlearrowright$ funt qui ferant potunc $\tilde{u}$ si= no calculosrodere, ficuti cum acelo puerorülichends.

Sylueitis coccimelea.

Sylusfris coccymelea fruftus, webementer adftrint git, adeo $q_{;}$O nentremretinct - Nominatur autembse plaittais Af fia Prumnum . Hortenfiacoccimelea, funt fimilia his que Damaf cena uocat Galcnus:

\section{Colla, Gluten.}

Gluten, cx fimiagine aut pollinc, emplafices atque concoctorise eft faculiatis.

\section{Colocyntha, Cucurbita.}

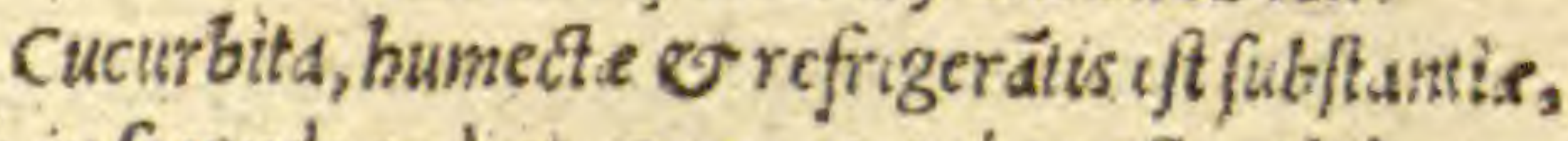

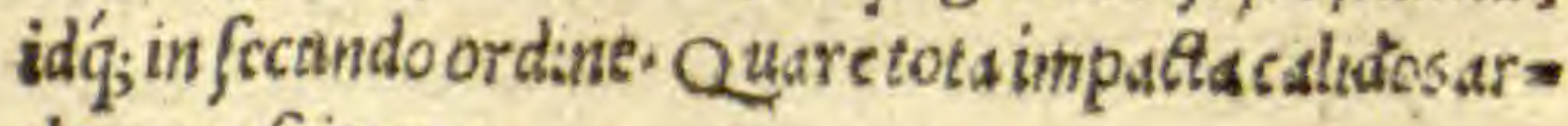
dores reffiger rat.

$F=$ Colccyansis. 


\section{P A V L. AE G I N E T A}

\section{Colocynthis.}

Colocynthis, Cathartice eft potentie uebcmëter, ui rides ipfius fuccus intritus, if chisdibus prodeft.

\section{Comarus, Arbutus.}

Arbutus,arbor ip $\int a$ qualitate acerba, una cŭ fructu eft-Momecylum quidam appellant, ftomachio infeftus eft, $\sigma$ capitis dolores excitat.

\section{Gummi:}

Gummi, arefacientis eft, or impactilis potentie, proptcred a pperitatibus midetur.

\section{Conia.}

Lixiuium, fic quod selut ablutio off cineris nomina tur, plus omnibus uim habet abftergendi, atg; arcfaci= endi. Inprimisueroque ex ficulncorumlignorü efl $c i=$ nere, ueletiam ex tithymallis, o feréque dicuntur pu tride potentie. Quod i quis calcis quoq; dabibuerit ci nerem, luxiuium faciet caufftcum at q; uehcmenti $\beta$ imü, - quod clyftecis mifcetur medicamentis, acerrtmum tamen omnum quodex folacalce fit.

\section{Coniza, Pulicaria.}

Pulicarie ambe, ficcant o calefaciunt in tertio gra $d u$. Qu o fit ut oleüin quo decocte fucrint, rigores qui percircuitum redeunt. fanent. flores quoq; earum ad = fimilis funt facultatis, quorum decotum, tum Mienfics ct, tumembrya deicicit.Porrò que in humidis locis pro 


\section{DE S I M P I I CIB V S.}

uenit pulicarid, ut prioribus minus bene olet, ita potens tie of t mbecillioris.

\section{Stercus.}

Stcrcord in uniuer fum quidē omnid calida funt, $\mathbb{E}^{\circ}$ maiorem immodü ficce $\odot$ degercntis potentie, immu tantur $q_{j}$. ecundum exccrnentis animantis temperamen tum 0 dietam.

\section{Stercus caninum.}

Stercus canum, corum maxime quioßibus uefcuns tur, ficcatum, dy fenterias fanat, nimtrü cum lacte fchi foo potum, ideft, $m$ quo egregatume $f$ f fcrum dicd $f e 0$. Preterca pharmacis bulcera uetufta ficcantibus, com= mode mifcetur. Anginis ctiam o $\sigma$ parifthmijs cum mel le intritum, admodum prefenti remedio inungitur.

\section{Stercus pueroram.}

Nec diucr $\int a$ factt puerorüftercus, priuato uictu ad bec nutritorum.

\section{Stercus luporum.}

Stercus luporum, illud quodcandidius eft, dtque in fruticibus reperitur, termino os magnifice iuuat, non potum modo, uerumetiam catapla fmatc impo fitü. Ap= pendi autem hoc uolunt éland, ouis uidelica à lupode= uorate, uel expellis ceruina loro.

\section{Stercus caprarum.}

Caprarüftercus, acris fimul $\mathcal{O}$ digerentis poten= tie, quoslibet induratos fcirror $\bar{u}$ tumores digertt, $c a=$ caplajmate illtŭ, uftumabftergentius, pariü q; magis tenuium fit.

₹ 3 Sterctus 
P. A V L. AEGINETA

\section{Stercus Boum.}

Stercusboum, quaddambabct attractorie facultas tis. Vefparum ttaq; alq; apum morfus fanat - In morbo intercutaneo, fi ques totum corpus bubulo ftercorc in foleoblinat, frenute auxiliatur.

\section{Stercus Ouium.}

Ouium ftercus cum aceto, myrmecias, of acrochor ddvas, $\mathcal{O}$ furunculos, fure claulos fanat.

\section{Stercus columbarum.}

Stercus fylueftrum Columbarum, acrimonia fter= coribw: alijs prepollens, cum femine Nafturcij adbibi tum, rubificat.

\section{Stercus domefticarum auium.}

Domefficarum autunffercus, cum oxamelle, aceto, uel mul sopotum, fuffocantes fungos cuomerc facit, $\sigma$ fiexi pharmacum $s t$, o colisis quildan etiam potans damexhibent.

\section{Stercus Ciconiarum.}

Ciconiarum ftercus or hopnoicis mederi aiunt.

\section{Stercus Murium.}

Strrcusmurinum, quontamplurimum abftergit, alo. petids cirat, er pucrorum $\int$ cdi appofitum, ad excretio nem proritat:

\section{Stercus Sturni.}

Sturnorum fercus, fiquando oryzancomederunt,

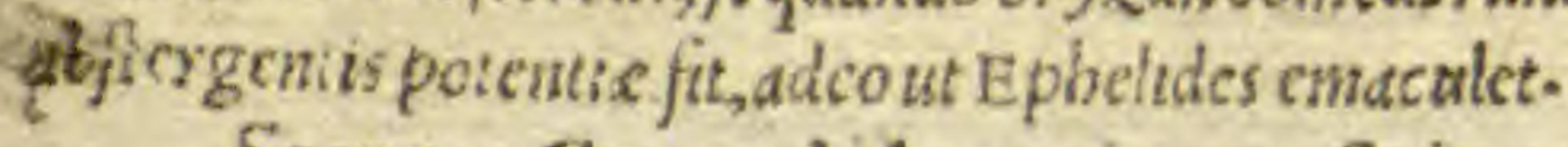
Stercus Crocodylortun terreftrium, Terreftrum 


\section{DESIM P I I CIBVS.}

Terreftrium crocodylor um fercus, impetigines $a b=$ fergit, co modo quo Nelicrocodytorum excrementum, Leucomata exteniare creditur.

\section{Corallium.}

Crefcit arbor um modo corcllium, of in duriciem Iapldis duratur in mari, colore rubeum, lapidi fimali.Fa culiatem habct exiccatoriam, ov adftringentemmedio criter, ob can: q, rem, bemoptokis confert $\mathscr{O} d y \int f s n=$ teritis.

Coris.

xógres anumah . Scribunt quidam quomodo cum dcso pobis, hirundenss cijciant.

\section{Coris herba}

Coris berbs, que o Hypericon, acris cdorate of;, ctuius femen bibitu, Nenfes o urtnam trabit - Sucurrit ophalangiorummorfibus, o in fapulas rigentlbus, cum olco inungitur.

\section{Corianum uel Cariandrum.}

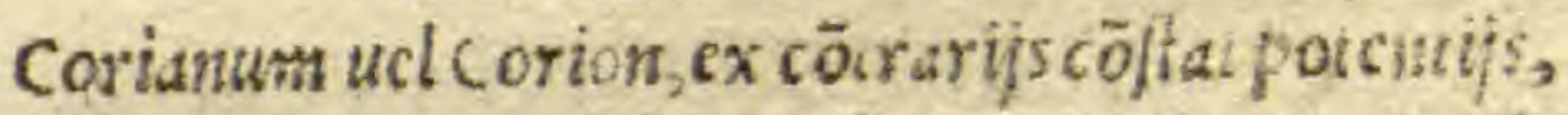

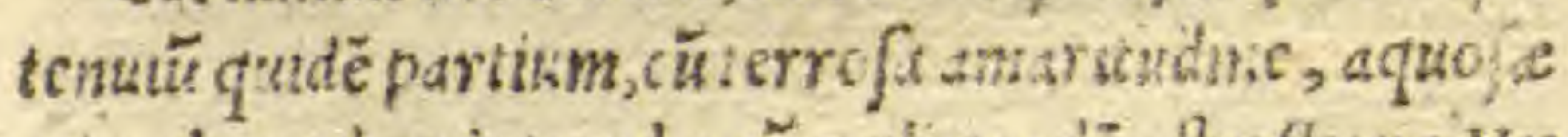
ctian bumuduat is teptde, cu cxeli quadẻ aftrifkonc.Vn dephicgnonbus. Orofipclutis sö admodü fcruenti= bus congruit. Delicatiores queg; tumores digerit, cum aliquo quodcommodeille p icutur - Semenlumbrices pelitt, parthm cum paffo fumptum. Maioritancricopia fump um, mentem non fice periculo tentet.

\section{Alauda.}

Corgdosucl coryidios, this cceda, fumplici in iu= F 4 foulo 
- PAVL. AEGINETA

fculocraßsioris inteftini, quod xó̀ $\lambda$ o $\mu$ Greci dicunt, do loribus medetur. Oportet tamen continuo, \& fepicule decobrodio edere.

\section{Coronopus.}

Coronopodis radix, $v$ ipfacommefta, torminofis opem ferrecreditur.

\section{Coftus.}

Coftus, paucule admodü eft amaritudinis, fcd acute - calide facultatis uehemèter. It tág; in qubus calef as cerepartem quamuis, aut ex alto in Juperficiem trahe= re eft opus, ad coftum confugitur. Eadem de caufa uri nas Menfesś; prouocat, $\sigma$ latos lumbricos occidit $\mathrm{Ha}$ bet quiddam etiam flatuofum. Propter quod Venerem

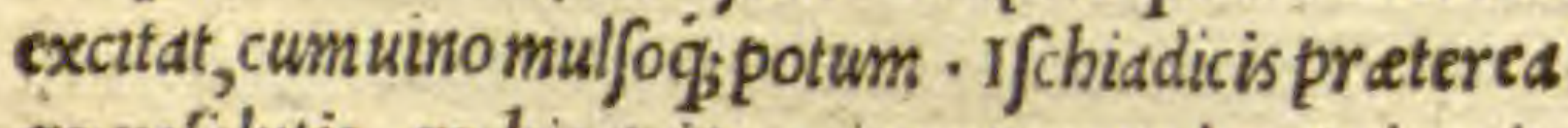
orefolutis, o bis quiper circuttum redeunt rigori= bus, cum oleointritum, opportunc inungitur.

\section{Cotyledon, Vmbilicus Veneris.}

Cotyledon, rcfrigerat er repercutit,abftergit $;$; $v$. digcrit, putantur folla cum radice manducala, calculos confringerc, $\sigma$ urinam cire.

\section{Cochlix.}

Cocblie ufte, ficce plurimum funt potentie, calide uerominus. Profunt dy fenterijsin quibus bulcera non dum computrefcere caperunt, fi in bunc modum mifcueris, uidclicet, ut Coch'eur m int partes quatuor, 


\section{DE SIM PLICIBVS}

piperis albi und, o gallarum partes due. Porrô Coch lie que uftionem non funt experte, trite, ovimpofite uentri, aqua intercutem laborantium, $\sigma$ in aribriticts sticulor $\tilde{u}$ tumoribus, quoad/ponte deciderunt, con= gruint. Nam fundtus, quicquid fuerit bumoris, ualide exiccdut.

\section{Cornus.}

Corniarboris fructusadftringit, of efui aptus eft.

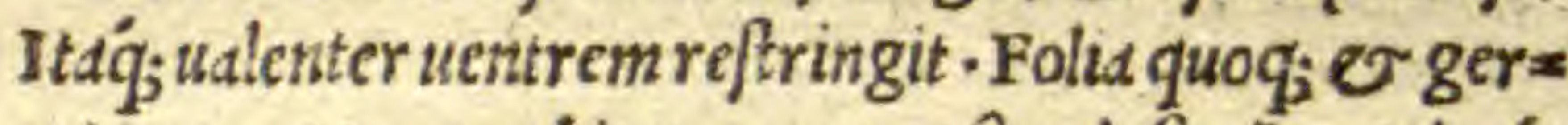
mina, proptcr acerbitatem impenfius deficcät, Adeo q o tulnera glutinarc ualsăt, potißimum durorumcor porum.

\section{Crambe, Braffica.}

Crambe cfculenta, deficcandi uim habet, cum efd, tum forisimpofitd, non tamen admodum acrem . Proin de hulcera glutinat maligna, o agre folubiles tumores fanat. Habet quiddam etiam in fe abfterforium, quole= pras curat. Semen etus potü, lumbricos latas interimit, - maxime Braßice Aegytia. Porrốcaulium combu= ftorum cinis, caufticam, fiue adurĕtem uim habet. Qua ratione, ficumadipe commifcedtur, laterum doloribus tnueteratis medetux.

\section{Braffica agreftis.}

Braßica agreftis, ad ommia pradtcta, horten/i, fiue fatiud, ualentior efl. Qudmobremneq; intracorpus, $\mathrm{Ci}$ tra noxam fumitur.

\section{Braffica marina.}




\section{P A V L. AE G I N E T A}

Marund Braßus, quiafubfulfa, dtq; fubamaraeft, wentrem deijcit.

\section{Cratxogonos.}

Crateogoniberbe fcmen, Ev acre guftantibus ef?, ov frigidum, fimile silio.

\section{Crethmon.}

Crethmon, fatfum quodammodo oft, cum patculte amaritudine, quamobrem uls eius eft exiccatoria, $\mathcal{O}^{\circ}$ abflerforis.

\section{Hordeum.}

Hordeum, refrigerat $\odot$ ficcat in primo ordine, $b d=$ bet ettam psuxilum abfterfionus, ev plus cxaccat, quàm farina fabarum, minus tameninflat. Caterum, Alphi= ton, hoc efl, polenta, mullio plus hordeodeficcat. Sed. Ptifanahumetat $\mathrm{e}$ abftergit magis.

\section{Crimnon.}

Crimznon, craffe e fentle, fic adpellatd, tum ex tri= tico, tum exzed farina, magis nutrut quàm polenta, mi=

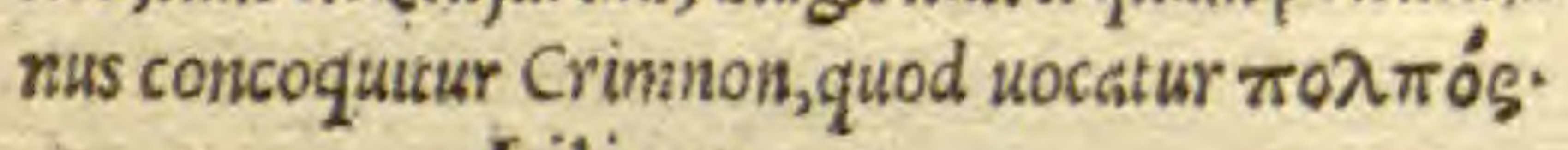

\section{Lilium.}

Lilij flos, temperaiuram mifticam obtinet, partim extenul, partim cx icrrena fubltantia, $\mathscr{\sigma}$ quadam $a=$ quofiate tcmperata confiluti-ltág quod cx cocompo= nutur oleum, digcrendi $\sigma$ emollende poicntic offici $=$ tur, uulua phlegnombus accommodum. Radix or

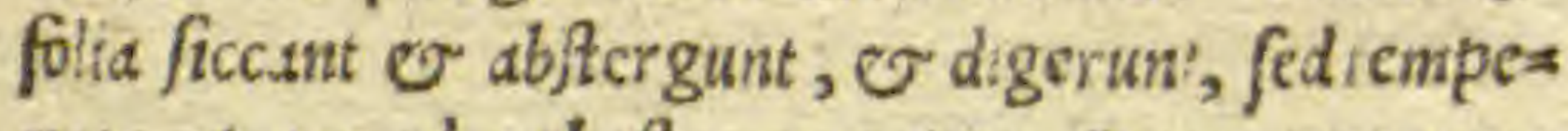
rate, quatre ad ambuftac competunt. Succus cum oxy" 


\section{DE S I P P I I I B V S.}

snclite, ncruorum incifioni duxiliatur.

\section{Crocodylion.}

crocodylij, o femen, o fuccus, ficce e digeren= tis fant potentia, urinam fimuler Menfes cient. Redix pectoris cxcreationibus conuenit, or per nares fanguss nem exirahit.

\section{Crocus.}

crocus, fecundarij quidem ordinis calefucicatioum eft, primi uero arefactentum, perinde fring:, o concoquit.

\section{Cromnion.}

Cepe, quidrto eft cale facicntiu ordine, effertiacraffs rum partium, unde hemorrhoidas appofita interrum = pit. Quaddamtenus etiam abftergit, quo fit, ut cum ace to alphos exterat, tum alopecias. Alcionio citius pilos reftituit - Porrô fuccus fuffu fis, aut pre craßitudune ds ctemoculorum obtufam babenttbus, duxtliatur.

\section{Cyamis, Faba.}

$F a b a$, nou multum à temperamento diftat, $t a n$ in cxiccando, quàminrefrigerdndo. Cdro quoq; cius po= tentie cft abfergentis. Excreationibus igitur, o fpus tis $\mathrm{ex}$ pulmone, $\mathcal{O}$ thorace, idoneum remedium. $\mathrm{p}_{u=}=$ tamen nommbtidaftringit. Tota fabacum oxycrato dc= cocid, coeltacts medetur, o dyffentcricis, cataplafmate iltta, phicgmonds fandt, 0 tib crd. In ulcribus quoq; Lic cxtingut.

Cyanos.

Cyanths 


\section{- PAVL. AE GINETA}

Cyanus, acri facultate $f t$, detrabendi $q u o g$; $\odot$ dige rendi, potenttori quam Cinnabdri - Ineft quoq; einons nulls abftrictio.

\section{Cyclaminos.}

Cyclaminos, uarle poteftatis eft, Etenimabsterget,

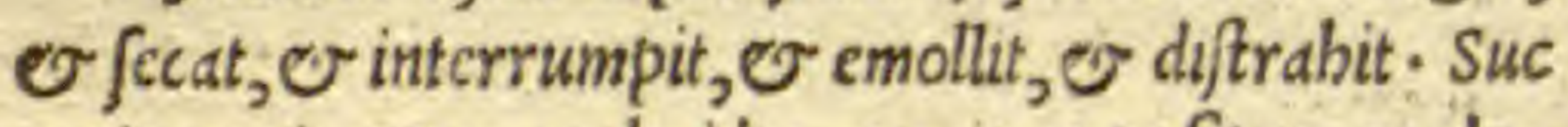
cus itaq; eius Hemorrhoidas aperit, appofitus, $\sigma$ du= ricies dif cutit, und̀cum alìss. Pretered $f u f f u$ is cümel= le conuenit, $\odot$ per nares expurgat, \& ill to uentricu= lo, ipfa uentrem fubducit, o embryısnecat. Tota autēe radix fucco quidé cf umbecillior, alioqui tamen $v$ ip $\int a$ webemens. Cum melicrato pota $*$ interanca expurgatAbftergit o que cutiuttiad dherent.

\section{Cyclaminos altera.}

Cyclaminos altera, quam $\mathrm{C}$ If ant hemon uocant, $\mathrm{rd}=$ diceminutlem habct, fed fruclum admodum efficacem, nec diuer fad prioriopcratur.

\section{Cuminum.}

Cuminum, cale faciends eft potentic, urinas cit, $\mathbb{O}$ flatus extinguit.

\section{Cinosbatus, Sentis Canis.}

Cinosbatt fructus, non fignter ad/frictoriuseft, folid uero mediocruter. Cauendumab eo, quod in fructucius lane fpecicmbabet, ceu arteriam uitians.

\section{Cyparifus.}

Capary ßi piule, o germina,ficce funt potẽntie, nullo tameninfignicalore, Vnde magnorum uulncrum induris 
in duriscorporibus funt glutinatoria. In humentibus quoq; putredinbus, ex altobumiditates innoxie depas

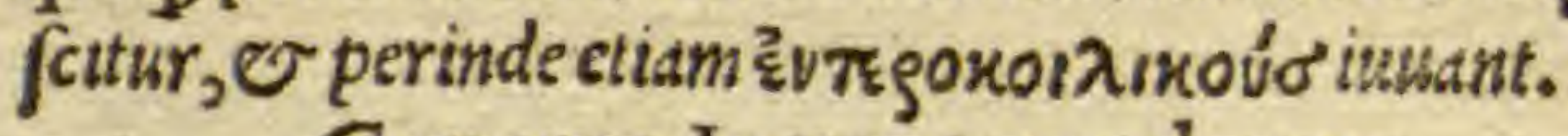

\section{Cyperus, Iu ncus quadiatus.}

cyperu, , calcfacit $\sigma$ ficcat fine mordicatione, qua = re bumida hulcera mir abiliter ad ctcatricem perducit. Hulccribus item oris congruit. Habet quanddm etiam incidendi uim, qua calculofis medetur, $\sigma$ urinas $\odot$ Menfes cit.

\section{Cypros.}

Cypri folia, fummaq́; germina, mixt funt potentie, adeo, ut $\mathbb{\sigma}$ digerant, $f$ ed fine mordicatione deficcantes, Itaqg; decocto corum aduer fum ambufta utuntur, et ad= uer fumignicas phlegmonas, o aphthas, comman[a. spleneticis porrô facultatibus commodemifcentur.

\section{Cytifum.}

Cytifi folia, fenfimdetrahendi uim habent, $\sigma$ tcpi= def funt potentie, quemadmodum o Malua.

\section{Comon, Cicuta.}

Cicuta, quòd extreme refrigerantis fit facultalis, nemininon cft compertum.

\section{Conos.}

Conicortex, of fila, potentia proxima funt pino, derioratamen $\sigma$ efficaciora. Fructum eius Strobilon uocant; qui, $f$ i uridus fit, quiddambabet amarum, cum dcerbahumiditate, propter quodex thorace laboranti = busidoneus eft. E/t auteme fui aptus, fi priusinaqua 


\section{P A V I. AE GINETA}

maceretur, ut acuitatem amittat, qua coctura fic tempe. ratur, utomnium minime mordeat, emplaficum q́a red ditur, er apperitatem omnem demulceat.

DE HIS QVAE INCIPIVNT

\section{abliterd $\Lambda$. \\ Lagopos.}

A A gopus, ficcatorie facultatis eft, $\bullet$ 104 ucntris fluxiones arcfacit.

1. 1 Ladanon.

Ladanum ex Cifto cyprio frutice, quem Ladons uocant $t_{2}$ colligitur - Siqui demcapre pafcentes, barbis ac focmoribus, quod pin= guc aclentori fimilc in eo oft, fecum aufferuint, id fóf collc csum, Ladanum eft, pharmacum efl, caldam ferè $f e$ cundoordine, partium tenuium, ex quo uires po ßstdet molihficandi moderate, digerendi, $\sigma$ concogisend, pro pter quod unluce paßsombus peculariter accomanoda tur. Habct quiddame etianiadfringens, quafluentes ca pillos retinet at q $_{5}$ compe fait.

\section{Lathyris.}

Lathyris $\beta$ ßecies Tubbymalli. Sunt qui fuccume eius aqualiter cumt th hymallo uelint purgare. Namde femi ne compertum eft , quod maximam uim purgatricem obinet.

\section{Lapfana.}

LAp]and, commefla quidem, malum fuccum giguit, ac pigmentatuabfergitac detrahit. 
DE SIMPIICIBVS.

\section{Lapathon.}

Lapatbum, digerenis medlocriter ef potentic.

\section{Oxilapathon.}

Oxilapathon, ures expellendi habet. Semen, pro= pter adftridionem qua mhabet, diarrhoeas $0 \mathrm{O}$ ayj]ens

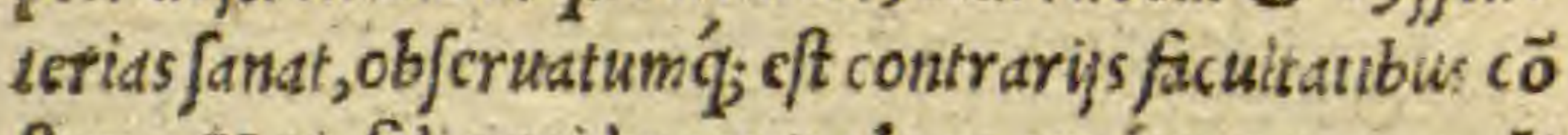
fure. Nam folid quiden exturbant, $\mathcal{O}$ lien rem mosĕt

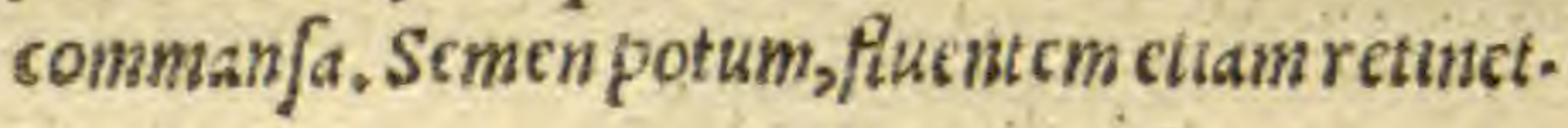

\section{Limoniam.}

Limorij fuccum ue'uti aufterum cum uino exbibent collacis, $\mathcal{O}$ dyffentericis, $\mathcal{O}$ bemopthoicis. I tuat $\mathbb{O}$ mulicbre proflumum, oxybaphuidclice mensura.

\section{Lichen.}

Lichen, qui uclut muftus quidam, petris adna fatur, abfergentis fimul of mediocrutter refrigerätıs eo exic cantis est potentie. Lichends igitur fanat, o inflam= mationes arcet, quemadinodumauthor eft Dioficorides. Et hemorragias quoq; fit/tit.

\section{Lichenes equorum.}

Equorum Lichenes, cum aceto rituratos, ferunt in primus Epilepfias, deinde $q 0_{j} \dot{\alpha}$ beftijsmorjos, pro $=$ be curare.

\section{Leontopetalon.}

Lecniopetalirdd: $x, \mathcal{O}$ ficcie intertioordine, $\mathcal{O}$ dis frahentis cft potentice. 


\section{P A V L. AE G I N E T A}

\section{Lepidium.}

Lepidiŭ,quod $\sigma$ Birlada wocant, extertio eft calefa cientium ordine, facultate $\mathrm{N}$ afturcio fimle, quanquam minus co exiccet.

\section{Lepis,Squama æris uel ferri.}

FE Omnisfquamaualidedeficcat, $\sigma$ ftringit, frenue etiam mordax.

\section{Squama rris.}

Squama er is, uebementius ficcat, partium q; eft ma gis tenuium. Quiddametiam habens eruginis, maxime que ex Cypriorü eft clauis. Vnde $\mathcal{\sigma}$ Heletim edm uocăt

Squama Ferri.

squama ferri, plus habet adftrictionis, o priorema iorcm ftomomatis. Quare bulcera, que cacoethe no= cantur, melus mundıficat . Carnem quoq; potcntius li= quat, quàm quelibet fquama eris.

\section{Leucacanthon.}

Leucacanthon, quam alï Poligonaton, alij 1 fchidds wocant - Huius rad ix incidat $\mathcal{O}$ arefacit in tertid diftan tia, excalfacitueroin prima.

\section{Leucas.}

Leucas, calc facit quidem $\sigma$ exiccat intertio ordis me, fed predominatur in caacrimonia.

\section{Leucoion.}

Leucoij, frutexin totum, abftergende potentie $r$ ft, - tenuium partium. Id tamen umpen fus faciunt flo= res, E horumaridi, uiridibus impen fus. Proinde men 
DE $S I M P L I C I B V S$. $4 t$

ftrua cit, \& fretum abfumit atgs proculcat - Ouod fi quis immixta fubinde aqud, thircs euts hebetallerit, the flanmationibus remedium prafentancum fuerit. Ante omnis uero mulcebribus locorum ticijs, Radices pro= ximas, atg; equales uires poßident, ettamfi partiüma=, giscraßtorum, O plus fubftantie ierrene participêt, ex quo indur atas phlegmonas medicantur.

Leuce.

Leuce arbor, exaqueatcpida o tcrrena fubltane tiaconfijtit, abstergentis potentia particcps.

\section{Libanotides.}

Libanotides tres funt, una fine fructu, fructifere due, omnes abftergentes ev difcutientes, o mollendi potentie : Q gorum fuccusctum melle mifus.obtufam oculorum aciem, ab humorum craßtitudine emendat. Porró eius, que ad coronas eft utikis, decoctum, regio morbo laborantes adiunat.

\section{Libanotus.}

Libanotus, calefacit quidem in fecundoordine, $c x=$ iccat autem in prtmo. Tamet $/ \bar{i}$ aliquaniulum quo oq; $\sqrt{u} b$ firingat-Alioquicortex adfringı manifetario, $\mathcal{O}$ are facit in fectunda diftantia, multo cra Siorü partisum quä thus ipfum etiam/i minus acrimonie habcat.1iag; be= moptotcos adiunat, nö folumintrinfecus appoftitus, fed ctidam afjumptus ad corports interiora.Germen eius ca le facti $\mathcal{O}$ ficcat in tertio ordine. Habet uero ettă abjler foriü quiddaim. Itaq; eo nomine expurgat o implet, que in oculis funt hulcera. 


\section{PA V L. AE G INETA \\ Lignys fuligo.}

Fuligo, quarundam ardentium $\beta$ pecierum quaficis nis quidam eff, ueluti libanoti, myrrhe, erminth, fty= racks, picis liquide, $\mathcal{E}$ cedri, tempctaméto ficca $\mathcal{O}$ ter Yofa, $\mathcal{E}$ partum tcnutum. Sed que $\beta$ peciattm in ea diffe rentic funt, es pro uffa materia cuentüt $v$ for pant aüt ca poti $\beta$ trum ad ophib ilmicas facultates, quas uocants

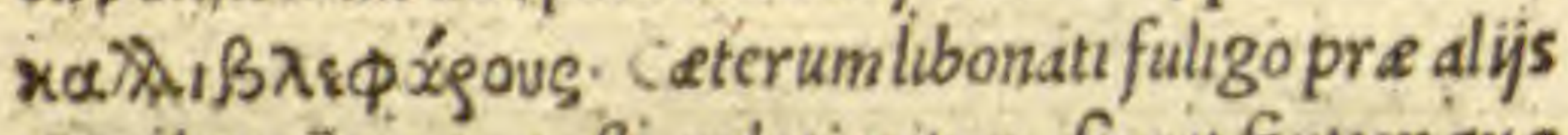
om mibus cömodior eft, ueluti mittor, ficcat fortior que iff ex liquida pice, $\sigma$ piced $Q$ unare ad deflunia chlor $\vec{u}$, - ad angulos oculor umbumentes, ero fos q́; utuntur.

\section{Ligufticum.}

Liguftici, O radix of femen excalefaciětibus eft,

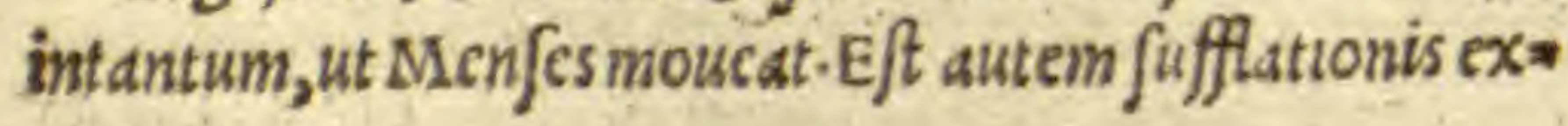
pers.

\section{Litargyros.}

Litargyros medy unter metallica eft ordinis - $\mathrm{Qu} d=$ prop er ianquam materia co utimur perfepealijs mt* fcentes facultatibus. Siccat autem mediocriter abfter = git $q_{;}$\& ftringit - Inde ad femorumintertrigenes uttilis eft medela.

\section{Lapides.}

Lapides uniuer fi qudè exiccant, perinde ut terre. Hamatites.

Hematites ft ingut of ficcat uchementer. Quare aperitatibus oculorum congruit. Hijs qudem qut fine pblegmone funt, cum aqua, cum phlegmone uero cum 


\section{DE SIMPLICIBVS.}

ouo, opitulatur $\mathcal{O}$ fanguinë expuentibus. Hulcerumq́ carnes er fuperexcrefcentias reprimit.

\section{Schiltos.}

Hematiti lapidı fimilem quidem, fed debiliorcm un habet Schiftus nomine dictus.

\section{Galactites.}

Galactites, eadem poteft que Scbiftus.

Melititis.

Melititis, quiddam babet caldditakis.

Myeroxos, uel Moroxos.

Myeroxik, quem er leucographida nocant, quinto memoratts mollior eft, mages q, qualitatis expers, eo temperatior $\sigma$ doloris magis experseft. Q u cerato ip fum mifcentes ad cicatrices perducendas bulas ccrum, in mollbus eo utuntur corporibus.

\section{Iafpis.}

subuiridis Iafpes, uchementior is potentie cum fit, cicatrices $\mathcal{O}$ ptery gia attenuat.

\section{Iafpis Virens:}

1afpis totaliter utrens, ftomachum corroborat, tam appofitus, quàmin digitulo portatus.

\section{Lapis Iudaicus.}

Lapis Iudaicus, que fic appelltant, inrenibuis lapi=

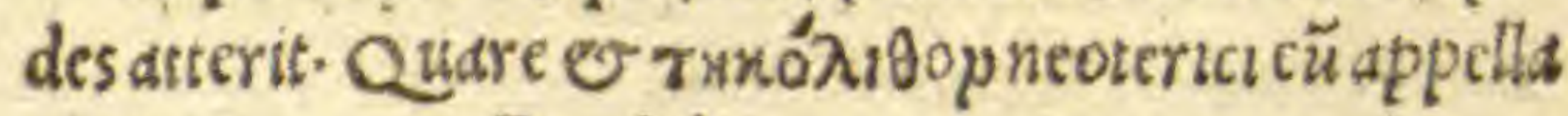
ucrunt. Pyritis.

Pyrites, ex numeroeorū, qui ualide digerŭt, tumo $=$ res o fangunis grumos - Quitamenfi forte in pard= to nonbabeatur, proeo Mylute utuntur. 


\section{P A V L. AE GIN E T A}

\section{Phrygius.}

Phrygius lapis, cum hoc quod ualde arefacit, quid $=$ dambabet etiä acerbitatis $\mathcal{O}$ morfus. Vinde $\mathcal{O}$ expellit - diftrahit,optbalmicisq; mifcetur facultatibus.

\section{Ageratos.}

Ageratuslapis, adftringentis $\sigma$ difcuticndi poten tic, prodeft cum primis gurgulionibus $\odot$ guturitna fluminato.

\section{Lapis Afius.}

Afie, petreflos, partcumtentium cf in tantum, ut carnes gractesliquefactat of falfas reddat, citraualie dum:morfum.

\section{Cagates.}

Cagates, lapis uchementer ficca potentie, inflation mibusconduct, maximecbronicts.

\section{Magnes.}

Magnites, qu 0 Heracha equalem hematiti facul tatemhabet.

\section{Arabicus.}

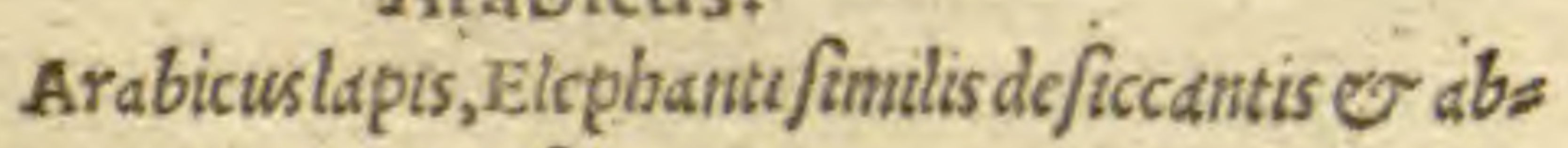
ftergentispoteniie eft.

\section{Alabaftrites.}

Alabaftriten, combuffum quiddm in potione propi nant ftomachutis.

\section{Smiris,} rificat.
Smtris, abftergentem babet facultatem, decođus pu 


\section{DE SIMPLICIB VS.}

Lapides qui in Spongijs.

Quimpongijsinuemuntur lapides, calculos in re= nibusconterunt.

\section{Lapides in Argxo Capadocix.}

Ginguntur or in Argeo Capadocie montclagides, Bpongiofispotcrituadfimiles.

\section{Ophites.}

Ophites, uocatus lapis, quiadalligatus, duiperamor fis auxiliatur.

\section{Oftracites \& Geodes.}

Of traciten $\mathcal{O}$ Gcoden ficce potentie effe ferunt; to inten $\int e$, ut ardentes tumores perfanct.

Naxias.

Naxe, cotis contritionem, rcfrigeratoriam habere potentiam ferunt. Ideo cre fentilus uirginum mammis $\mathcal{O}$ puerorumteftculis utilem effe.

\section{De olearia Cotz.}

Qunoducro exoleariacote detritum fuerit, nimir $\bar{u}$ propter abftergentem facultatem quambabet, alope= tias fandt.

\section{Hieracites, \& Indicus.}

Hicracites $\mathcal{E}$ Indicus; Hemorrhoidas are faciuntSaphyrus.

Creditur fapbyrus lapis fcorpionum ictibus potus aduerfari.

\section{Aphrorelinus.}

A pbrofelinus lapu comuatit morbouscxtos liberd= reputatur. Armeniacus. 
P A V L. AE G I T T

Armeniacus lapis per infernaucntrem purgat, fed cacoftomachus,

\section{Lithofpermon, Milium folis.}

$\therefore$ Lithopermon, id quodatij Lioporon. Atij Herd= cliumuocant, non Aetonychon, ab aquilarum ungui= bus, femen lapideum e album profert, quod potum cum uino albo ucfica calculos frangere $\mathcal{O}$ urinamcies repotefl.

\section{Linofpermon, Linifemein.}

Linofpermon, caldum ef tin tertio ordine, at bumi= ditatıs $\sigma$ ficcitatis quodammodoin medio cöftitutum. MManfum flatuo fum eft, etiamfi frigatur.

\section{Linozofos Mercurialis.}

Mercturialis, uentrè fubduct efficaciter $\odot$ pigmen tata digerit.

\section{Litrum.}

Litron A phronitri, $\mathcal{O}$ falium naturam h bet, uffii tenuium parttum, $\mathcal{F}$ magis digerentis facultatis red = ditur . Corporililitumincidit $\mathcal{\sigma}$ extenuat crafos $\mathcal{O}$ Icntos humores, plus omnibus alijs. Porrò Chalaftreon exproutntia Tefsalonica, chaleftra acrius $\&$ ficcius cft. Qutare ad deftillationes capitis, $\odot$ arthritica eo situntur.

Lonchitidis.

Lonchitidis, cius utdelicet que femen habet triangu Lure, cuppidis figura- Radix aquales folopendrio uires bisbet, folla uirtida unlnera conglutinant. Arida ueroc $\bar{k}$ asctopotd, ,plenibus medentur induratis. 
DE SIM P I I CIB V S: 44

\section{Lycopfos.}

Lycopfi, radicem adftringentem qualitatem babes re,teftis eff Diofcorides, $\mathcal{E}$ cumpolenta quidem cōfar ire crefipilatis. Ceterümtritam cxolco, ev corpcribus: nunctam, fudorcs care.

\section{Lycium.}

Iycium ex diuer fis conflat fubftantijs, alierd qui $=$ demtenuum partium digerente, altcra terreftri o for gida.Senfum etum ad/fringit, unde deprebenditur fice care in fecundo ordine. Calefacit ttaq; $\sigma$ refrigerat me diocriter, propter quod ob uim cius abftergenté, utuns tur eo ad medccinas, que clartatatiof ficuut oculorum opupillorum, $\mathcal{\sigma}$ ratione ftriclure ad malum cocliacum - dy/enterias, $\mathcal{O}$ hulceramale habitudenis, poffrcmo o ad phlegmonas, proptcr difcußtonem. Prafertur ad hec omnis Indicum.

\section{Lyfimachium.}

Lyfimachium, pradominantem babet dcerbam qud litatem, qua quidcm uulnera glustinat $\mathcal{O}$ fanguinem re tinct, tam ip fa quam fuccus ceus. Pota fubinde uel unie= Cis dyfenterte pref contancum remedium eft.

\section{Lychnis.}

Lychnidis, coronarie femen, calldum eft at ficcum in fecundo ordine complete.

\section{Lotos domeftica.}

Lotos domeftica, $q$ a aly tryphyllon nominant, mez diocriter abftergends $\sigma$ ficcandipotcitis, uel potus caliditate temperdid. 


\section{PAVL. AE G I NETA}

\section{Lotus Agreftis.}

Lotifylueftis femen, fecundis eft calefacientium ordi. nis, habetf; quiddam abfergens.

\section{Lotos Aegyptia,}

Loti Acgyptie femen, huulfmodiest, ut exco.panis fiat:

\section{Lotus arbor.}

Lotosarbor, tenutü partiumeft, $\mathcal{E}$ ficsans er ad = ftringcns mcdiocriter, itaq; accommodatur profuutijs, mulicrum, 0 coelacis affectibus, fiue in aqua uel uino pota, fiuc etiam infuga. Fluestes quoque capillos con * firmat.

\section{$D E H$ I IS Q V AE I N cipiunt ablitera $\mathrm{M}$.}

\section{Macer.}

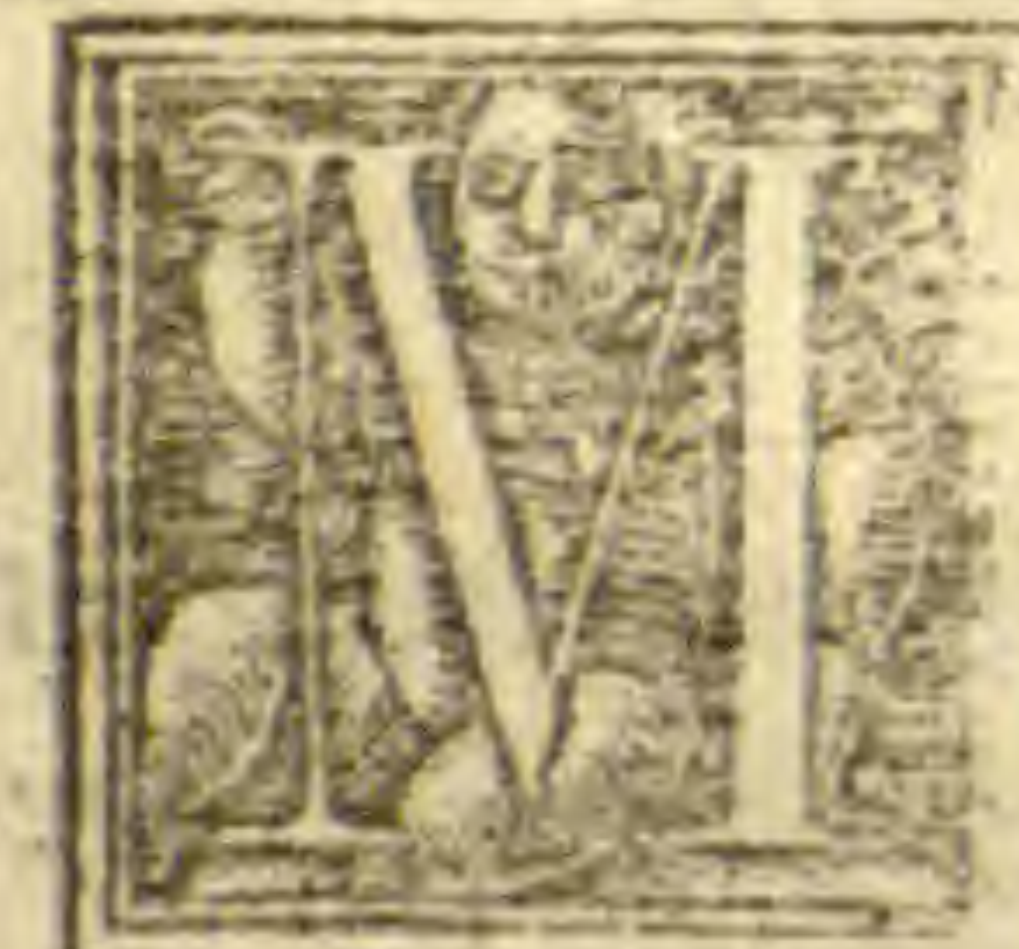

Acercortex, qui ex India delatus, Sic= cat in tertio ordine, mediat $q_{;}$quodam $=$ modo inter calditatem $\sigma$ frigiditate. Accrbus preterea, $\mathcal{O}$ partium tenutu. Prounde coeliacis ac dy ffentericis congruit.

Malabathrum,

Malabatbrüfohü, poteftate nardiarifte eft fimile. Malache, Malua.

Malua, fylucftris, diffrabêdi er molliendi facultatë babet. Hortenfis quatenus plus bumiditat is admintit,

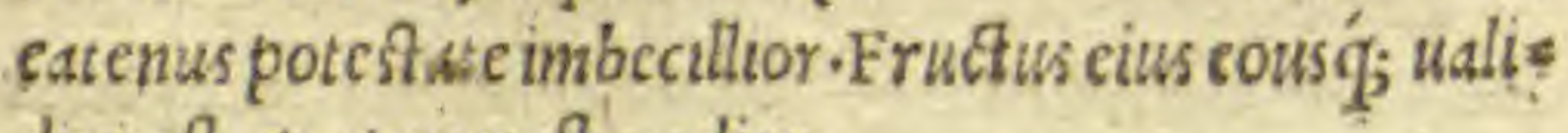
dior eff, quatenus if aridior.

Mamiras. 


\section{DE-S I M P I I CI B V S.}

Mamiras, radix off quedam herbe digiti cruß $3 i t u=$ dinis, que cicatrices $\mathcal{O}$. Letucomata attenuarc credite eff, manifefteabftergentis posentice.

\section{Mandragora.}

Mandragora, tertij eft refrigerantium ordinis. Ma la ueroeius caliditatem quandam $\mathcal{V}$ humiditatem par= ticipant, quare fommum conciliandium habent, Radi= cis autem cortex, $\mathrm{cum}$ fit uslidißimus, non modo refrige. rat, uerumetiam arefacit. Arbor ipfa imbectllior eft.

\section{Manna.}

Manna Thuris, Thuricafdemuires habet, inefficas tiores tantummodo, $\sigma$ enfim adftringentes.

\section{Marathrum.}

Foniculum, calefacit in tertia potentia, are facitues ro in prima. Proindelac gignit, o codem modo $f u f f u=$ fisquogiduxiliatur.

\section{Maftiche Chia.}

Maftiche Chia, cale facit $\sigma$ exicsat in primo gradu. Compofita quodammodo ex contrarijs potcntijs, acers befcenti, o mollienti. Ideoflomachi, e d alui, o intefti norum, o iecoris conuenitinflammationibus.

\section{Maftiche Aegyptia.}

Maftiche Acgyptia nigrior, tum cxiccat amplius, $t \tilde{u}$ difcutti munus, furunculos digerit.

\section{Melan Indicum.}

Melan Indicü, ut inquit Diofcorides, ex fucillimere frigerätıbus eft, phlegmonas q́; e ocdomata rüpétibus bulccraremouentibus:

G, Melan= 


\section{P A Y L AE G I N E T A}

\section{Melauteria.}

Melanteria, ftringut uchementer, eff $\dot{q}_{\text {; }}$ drtium tez nuium.

\section{Atramentun fcriptotium.}

Atramen ium fcriptorum, priorıma gis de ficcat, $O^{\circ}$ sum oxicrato pericauftis inunctum, ituat.

\section{Melanthum.}

Melanthum, cale fact, $\mathcal{O}$ are ficut uxid atertium or * dincm, $\sigma$ partumtenuum eft - Quireodoratum, cd tarrhis, fute deft tllationibus medetur. Intra ctiã affum

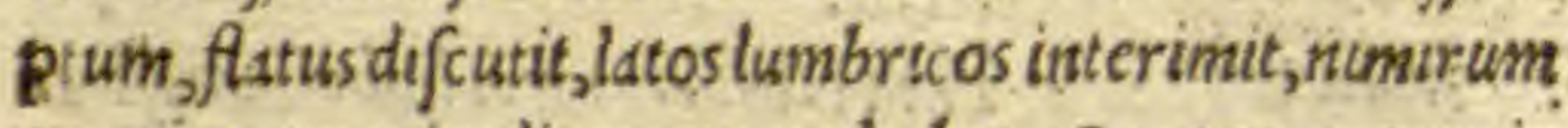
propter amaritudinem quam babet. Preterea quoniz am abftergit, $\mathcal{E}$ incidit,arthopnotcis porrigitur, of le pras abfergit. Ad poftremum myrmecias exterit, $\sigma_{2}$ menftruacommout.

\section{$\mathrm{Mel}$.}

Mel, calcfacit $\mathcal{E}$ exiccat in fecundo ordine, $\mathcal{O}$ inter Atrenue extcrgenit numeratur. Coctum tamenninus acre, abflerforcumq́; reddeur, aduentrem fubducendü, cum alloqui plus nutrat.

\section{Mel amarum.}

Melamarum, $\sigma$ quod n Sardinu gignitur, mifte off potentie, terrce uidelicet, $\mathcal{O}$ calefictentls.

\section{Sachar.}

S.char, quod ex foeiccl Arabia dc fertur, minus quits demnoftratidulctus, fid ad fimules tamen utres obunet, neqg; cacoftomachum, neq; fium affercus. 


\section{DE S I M P L I C I B V
Melia,MalusPerfica.}

Melia, nota arbor, cuius folla pota, duiperd mor fis auxiliantur. Cortex ufus, $\sigma$ cum aqua illitus, lepras adımit. I ignitamen ramenta pota, letalla effe frütur.

\section{Melilotum.}

Meliotum, mixis eft potentic, acerbefcentisuideliz: cet, $\odot$ diffrabents,, concoquentis.

\section{Meliffophyllon.}

Meliffophyllon, confimilem prapio potentian? hase bet per omnia.

\section{Memæcylos.}

Memecylos, corniarboris fruetus, de quo antedi: ctum of in litera $\mathrm{K}$.

\section{Mefpila.}

Micpila, qua E Tricoced - Huius arborisfruAus admodum acerbus eft, adco ut edi üix ualeat - Proinde uentrem compefcit, Quir o rami o foliaeddem qua litate predita funt.

\section{Medion, Trifolium odoratum.}

Mediy radix. femini contrayjá uimbabet - Nambac quidem duftera cum fit, flaxiones retinet . Ceterum, $f e$ men ueluti partium tenuium, $\odot$ facultatis incidendis. Menfespropellit.

\section{Papauer, Ryas.}

Eftetiamnum Papatucr, quod Rhoeds nominatum eff, $x \times c 0$, quid confeftum defuit . Huius femen ualidius refrigerat quàm Hortenfe.Nequaquamtamen innoxie editur 


\section{P A V L.AE GINET A}

editur, cum alioquimelle plurimo miftũ, reddatur optie mum. Itemaliud eff; quod incapitulo femen nigrum continct, medicamentis aptum, fed uchementerrcfrize= ratorium. Et tertium pralongis capitibus omnuum

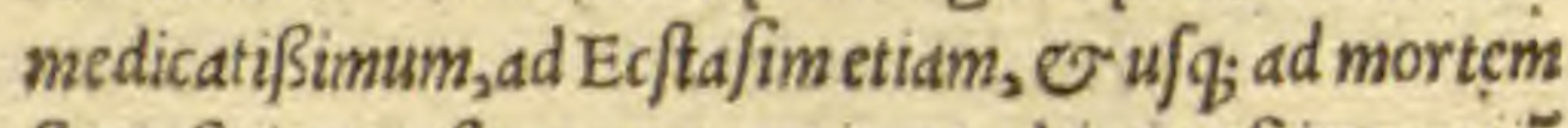
ftupefaciens, eft nanq; quarti, ov ultimi refrigerantiü ordinis, $\mathcal{\sigma}$ ip $\int u m \in \mathcal{\sigma}$ fuccuseius, quemuocant, perexs cellentiamopium.

\section{Papauer cornutum.}

Cornutum papauer, abftergentis cff potentic, qud = re radicis eius decoctum potum, hepaticos iuuat. Porrô folid $\odot$ flores; fordidahulcerareftaurant, $\mathcal{O}$ efcharas anferunt.

\section{Papauer Heracleum.}

1. Heracleum, E A phrundes uocatum papauer, femë habet, quod pituitam expurgat.

\section{Mala.}

Malis omnibus excrementicia $\odot$ frigidainef thumi ditas- Et alla quidem bumide fubftantie funt, frigidiora or bumidiardalic - Rurf fum alla dilcia aquofitate qui= dem non adcomanifefte frigidd. Alia aufter a, frigidios ra quidem pre dulcibus, fed minus humeda - temaceta $f t, \mathcal{\text { ip }} \int_{\mathrm{f}}$ frigidd, uerum in uentre bumorum incifiuds

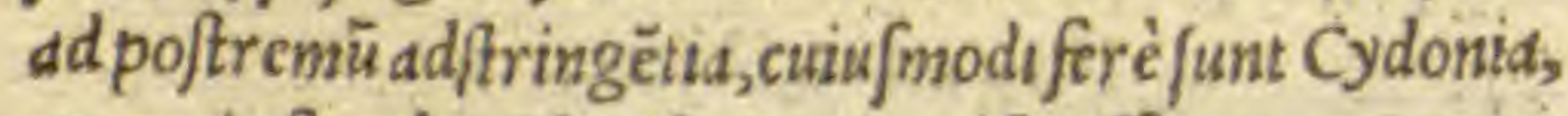
o mala ftru bia frigide o terro fe efcentie, propter quod uentrë fijtunt, et uulneracōglutinant- Ineü modü folla, fuccl, ct cortices, aliarum arborü inter fe dißjident. 


\section{DE SIMPLICIB VS Malus Perfica.}

Mall Per fice, germina of folia amare qualitatis ma niffte funt - Ideotrita, at q g uentrifuperimpofita, uer mes necant. Cateram, fructus eius, quod Per fieumno= minatum, \&f culentum, temperamento humidum eft, $\sigma$. frigidum in fecundo ordine.

\section{Mala Armenica.}

Mali Armeniaca fructus, quos uocät Precocia, Per ficorum funt potentie.

\section{Meon.}

Meu radices cale faciunt in tertio ordine, or exiccät iufecundo. Vrinam igitur mouent, of menftruacient. Si plustamen fump fris, quain par fuerit, capitis dolo= res exufcitant.

\section{Milax.}

Milax, tum Tela, tum Trachia, arboribuscircum= uolutur. Calefacientisq́; $\sigma$ acrioris e/t potentic.

\section{Mify.}

Mify, ex bis eft, que rehementer incruftant, $\sigma$ cals ftica funt, proinde ualde adjtringtt. Eiufdem ferè facul tatis, Mi $\int_{3}, \mathrm{P}$ fori, $\mathcal{O}$ Chalcits . Caterum Mify chalci $=$ tide minus mordet, etiamfi partium magis tenuium.

\section{Molybdena.}

Molybdena, fimtem Lithargyrouim poßidet, tan= tumà media temperic, ad frigıdius paulü qutdrecedens. Molybdos, Plumbum.

Plumbum fac ultatis cf treffigeratorie, habet $q_{;}$plu= 


\section{PAVL, AE G INETA}

simum effertichumide d frigorecodcie. Trito igitur in plumbimortariolo, fuccuno, fiue ole ${ }_{2}$ uel quotibet alio fucco, quirefrigerare debeat, ad phlegmonixs bulce ratas, qua in pudendis, aut circa fedem protuberät, me dicamentum commodü efficitur. Quin E lamina plum bilumbis adligata, uel inftrata, fluorem feminis in fom * nis cobibent. Sed $\sigma$ Bracteatenuis, ganglijsipfis ap= pofita, diffundited.

\section{Plumbum uftum.}

Plumbum uftum, ficcius eft, $v$ quiddam habet dcre. Elotum fine mordicatione äeficcat, 0 malorumbulces rum optimum eft medicamentum.

\section{Morea, Moruc.}

Morum maturum, uentrem fubducit: Immaturum, ubiarucritadmodum adftringtt. Cortex arboris, radi $=$ cis uim purgands babet, cum quadam amaritudine, qua re o latos lumbricos perimit . Folijs uero o germint= bus media quodammodo eft fubducentum o retinen= tium facultas.

\section{Myagros.}

Myagri femen craffumeft, $\odot$ quod in feoleofumba bet, emplaftice eft potentie.

\section{Myaces, pifces, uel Mituli.}

Myaces uft $t$, eandem facultalem habent cum bucci= nis, priuatim tamenablutia aperitatem palpebrarü, $\sigma^{-}$ albugines oculorum, cum melle abstergunt - Canü pres = teresmor fibus utiller caro corum imponitur.

Mytron 


\section{E S I M P L I C I B V S.
-Myaron, uel Myagros, herba.}

Myagros, quam aly Melampyron, fructuofa ber $=$ ba eft, $\sigma$ pinguis, crediturğ; femin s pinguttudo illa, cutis afperitatcs a quare omnt $s$, $\mathcal{O}$ leutgare.

\section{Myelos,Medulla.}

Medull, mollentis eft facultatis, induratorum edridorum corpor um, imprimis ucro ceruorum, deln= uttulorum, nam taurorum $\mathcal{O}$ caprarum acriores funt, $\mathcal{E}$ exucciores.

\section{Mitetes, Fungi.}

- Fungi,uchementer frigidi o humidi fun! temperd= menti. El quidam corum comeft, intcrfictunt, poußs a mum qut ex matura mis am habent qualitatem.

\section{Myos, wtis, Auricula muris.}

Auricula muris, cxplicata cft in dictione Alfine.

\section{Myza.}

Myzd, drboris cuilufdam fructus, longe minor Coca cymelis difts, ideft, Prunis.

\section{Myrice.}

Myrice, fecandl eft 0 abftergendi potentie, fine tamen deficcatione manififta. Habst vero quiddam. etiam nature adftringentis, $\mathcal{O}$ obid quidem euts de= coctum, $/$ plenes induratos fatts iuuat, dentium quoq doloresperfanat Ceterum, fructus $\mathcal{O}$ cortex, eiuf= demelt potenti- cuius eft Gatla. Cints pradictis omite bus ficcior eft.

\section{Myriophillon,}




\section{P A Y I: AE G IN ET A}

Myriophillon, exiccatorie of fecultatis, in tantums ut uninera glutinet.

Myrrha, uel Myrrhis.

Myrrbd, cx fecundo off calefacicintüu ordine, partiü habens nonnubiltenuium - Radicem babet non iucundi Thodo odoris, uerumetiamdulcem, que Menfes moutet, o que thoracemuel pulmoné pregrauatü expurgat.

\section{Myrrhine, Myrtus.}

Myrtus, excontrarijse escentysconflataeft, dominas tur enim in illa quiddam terrestre frigidum, propter quodimpenfeadmodumdeficcal- Porrô Myrtis, que

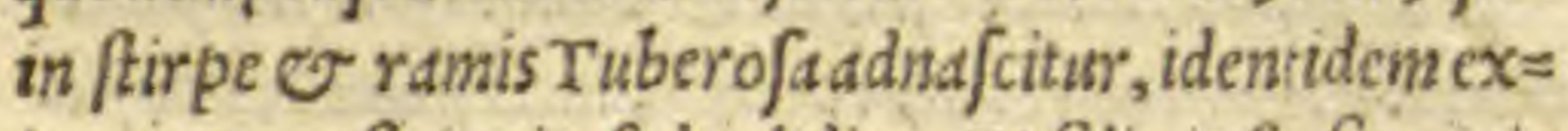
iccat $\sigma$ conftringit, fedualdius - Et follia ip $\int a$ ficca uis ridlbus plus arefaccunt. FruEtus itaq; $\odot \sim$ fuccus einfä́ funt potentie, fuce extrd, fuce intrain corpus fumpta.

\section{Moly.}

Moly, quam alquiuocant Rutam agrcfem, nonnul ti Armanain, Syriuero Befạ an, tertie eftecla Bis calefü cientium, cum partium tenuitate. Vnde ficcat $\mathcal{E}$ diftra bitcraffos $\odot$ tenaces bumores, urindm aduertit.

$$
\begin{gathered}
\text { DE HIS QVAE ICIPIVNT } \\
\text { dblitera } p \text {. } \\
\text { Nafcaphton. }
\end{gathered}
$$

Afcaphthon, fiue Nacaphtyop, Indicü 1. 1 eft aroma, adftriçus fomutarum locis 1. I. If fuffitum, uel euaporatum, prodeft.

Napy : 


\section{DE SIMPIICIBVS.}

dinem.

Napy, calefacit exiccat fecundum quartum ore

\section{Nardoftachijs.}

Nardoftachis, radix ralefacit quidem in primadi. ftantid, arefacit autem in fectunda: Conftat wero ex acer befcente dliquaniulum effertia or deuta, non admodü calidd, o quodam fubamaro bretai. Vinde pota er it $=$ cinori ef fomacho competit - Qutod fi extrdimponas, uel intro fumas, per potionem etismarinas moudt, in $=$ terraneorum fane fuxiones deficiat. Validior tamene adomnid eft Indica, quàn syriacd, ef mgra.

\section{Nardus Celtica.}

Nardus Celticd, proxime eft iam dicte potentie, $0^{\circ}$ incunctis inbecillior preterquaim ad mouendam uris nam. Alioquicalentior memorata fpica, minus uero acerbas.

\section{Nardus Montaná.}

Nardus montina, que er Thylacitis e Pyritis e/t dictd,predsctis imbecillior.

\section{Ferula, Narthex.}

Nartbicos, femen quidem extenude calcfacit, ue rum medulla adftringu - Propter quod Hemoptotis of coeludis axiliatur.

\section{Narce Thalaffa, Torpedo.}

Torpedo marina, caput dolentibus adbuc tiuess tins pofita, doloremarcet, Idq́feodemitlo tempore, quo mcmbra omnid, hominis cotactucius corporecredun= Mo: Bot. Gardenth tur.vir 


\section{P. V L. AE GINET A}

tur. Viués precerca in olco percocta at f; thundta acre. ar iculoram dolorcsml igut - Eadcm procideritum aù tnuerfam fcdem appoffiacoërcet.

\section{Narciflus.}

Narcißs radix, ficcantes eft potentice in tantumu uulnera glutinet, uel ad incifiones ufg, que circaten= dones accidun Habct quiddam etum abftergens 0 re uellens.

\section{Neuras.}

Neuras, quidam poterion appellant, ficcantis fine mordicationceft potentue Adeo ut neruos prectifos gitu tinire potis fir Idem fa cunt $\mathcal{O}$ radices tum umpofi.e; tumpote.

\section{Nerium Rhododaphne.}

Nerium, aut R Rododipne, , xtrorfum quidem cas taplafmatum, d gcrentis eft poientie. Intro ucroa ffum ptumuntericat.

\section{Nimphęa.}

Nymphea, umm habet arefuecédicitra uellicatione.

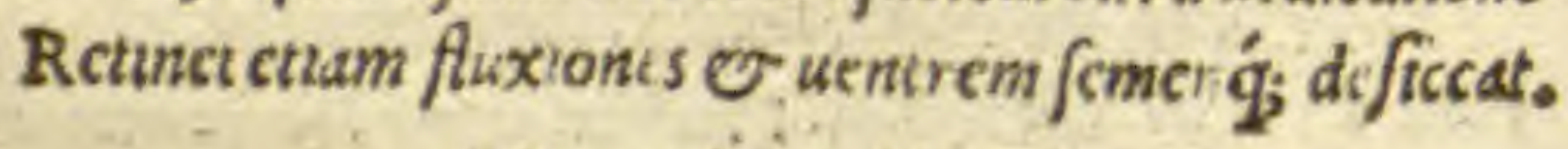

\section{Nimphęa Alba.}

Oue albam habet radiccm nymph a, uehementio riseft poteftatus, adeoutcum utro nigrouel aufteropo ta, form nar um $f$ ix us cobibcat: Porró que radicem $\mathrm{Ni}$ grambabet, abjtergendieft poicntle : Prosnde cü aquas attligtics 


\section{DE SIMPLICIBVS. 50}

titilligines exterit, $\mathcal{O}$ alopetiak cum liquida pice faitais

$D E$ H I S Q V AE I $N=\ldots$ ? cipiunt ablitera $\xi$

Xanthiou.

- anchion,quod alijphafganion, femêhas bet digerentis potcnitie,

\section{Xiris uel Xyris.}

$\mathrm{X}$ เristenuium partiü digerendi oo at $=$ tractorie eff facuiliatts, utdelicet fice e, fed multomagis femen, quod urinamcit of plenitndurato opitulaturs

D. H IIS QV AE IN= cipiuntablucra 0 .

\section{Othonnia.}

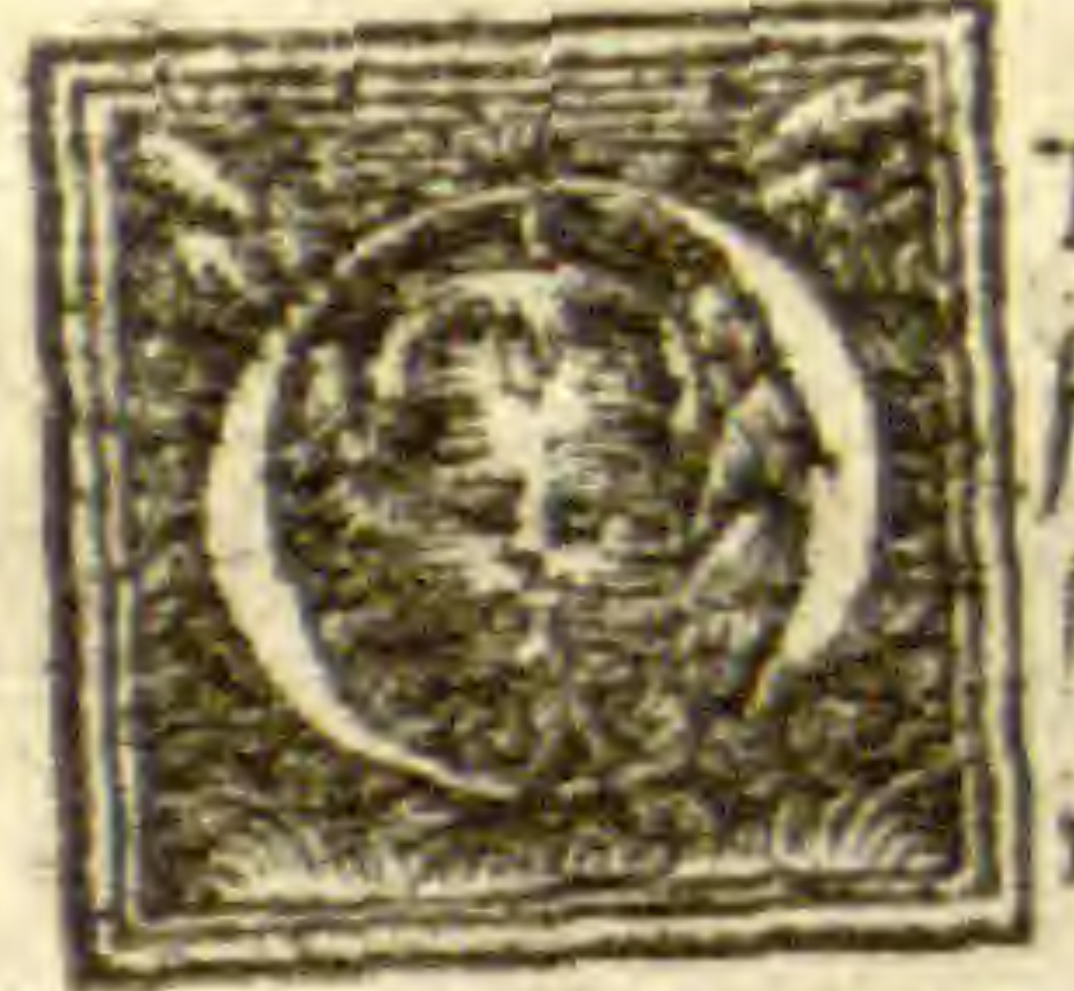

Thonniam, alqquiher be ciriujdam cffe fructum, in A rablacirca Aegyptŭ nas. centis. Aliy lapidem Aegyptum afje: runt, „abtergit o mordicat. Quamob rem que clartati officuint oculor um exterit.

\section{Oetnanthe.}

- Oenanthe, fylueftris uitisflos, cuilus admodum mul a this eft iffus, ueheméter adftrungu - Diofcorides altèra quandain de cribu contrariam potentiam babenté q que fecundas cijcere, ue fica fithicidia, $\mathcal{E}$ iciericism curdta recreditur.

Yinum,

$H \gg$ Vinum, 


\section{PA VL. AE GINETA}

$\nabla$ inumex fecundo ordine eft callefacientium $\sigma$ fics cantium, fed quod ddmodü uetuseft, ex tertio ficut mus ftum, quoddicunt exprimo.

\section{Oefypus.}

De Oefypo, in lanis fermo habttus eft.

\section{Olinthi Craffi.}

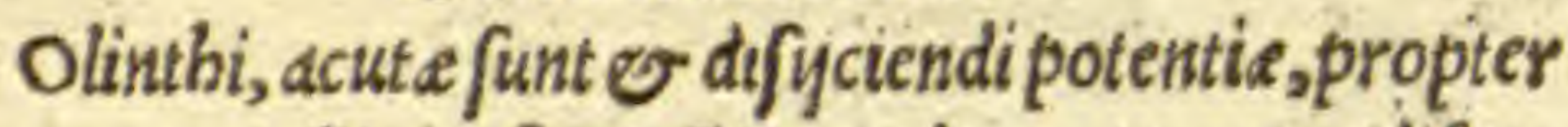
fuccum qui itlis ineft. Cottiitaq; duros tumores dif cu= tiunt. Crudi uero myrmetias $\mathbb{E}$ Thymos eijciunt.

\section{Oloftium.}

Oloftivin, deficcandi facultatem babet cŭ aftriftio ne-Quere ad rupta eo potisimum utuntur.

\section{Omphacium, Ex acerbis Vuis expreffus fuccus.}

Omphatium, fuccuseft une necdum maturefsentis, fringut uehementer $\odot$ ficcat in tertio ordine, quare fluxionibus o maximeftomachicis auxiliatur.

\section{Onagra.}

Onagran, alij onotheram, aliy onotburim uocant, grandis frutexeft $\sigma$ arborefcit-Cumus radix emplaftri modo impofita, dolofa or malefica bulcera lenit.

\section{Onoma uel Onofma.}

Onoma uel onomis, uel phlomitis, acris o d mara eft herba. Cuius follia cum uino pota, ex utcro foetü trd = bunt, e abortiua pregnantibus funt.

\section{Onobrychis.}

Onobrychis, rarefaciends $\sigma$ digerendifacultatem obtinet. 
obtinet. Phymatd igitur digerit $\mathrm{O}$ f frangurias fanat. Ad poftremum etiam fudores clicit.

\section{Oui Afini.}

Millipede, que in orbem fe agunt, digerëtis o fics ce funt potentis. Quare cum umo difjurijs 0 drqua tis fubueniunt-Synanchicis uero cum mellc anungütur, Infuper conct $f i s$ o in malicoro addito ro $\int a c c o c a l e f a=$ atis, moxóq; in aures inftillatis, corü doloribus medětur. Onyches, Conchula Indice.

Onyches, tefte funt Conchularü Indicarum. Que fuffite, ftrangulatione uuluarum concidentes forminds excitant, fimiliter quoq; epilcpticos pote, tamen whum turbant.

\section{Ononis.}

Ononis, radicembabet calidam intertio quodamte nus ordine. Cortex eius abftergit 0 incidit, quamob= reme lapillos confringit.

\section{Acetum,}

Acetum, mixte cft potentia, rcfrigerantis uidelicet

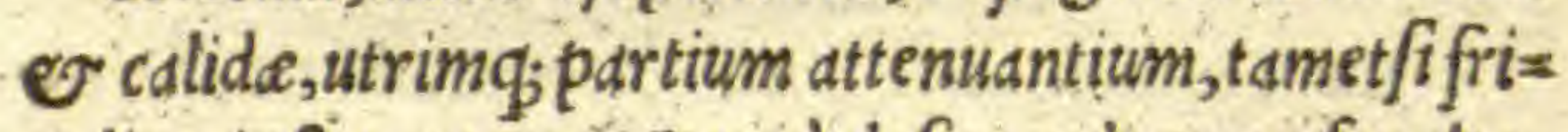
giditas ipfa exuperet - Porró de ficcandi quoq, faculta $=$ tem obtinct, que in tho predominatur, ufq; in tertium gradum.

\section{Oxyacanthos.}

Oxicanthos arbor, piro fylueftrifimilem tum $\beta e=$ ciem, tum qualitatem habct, quiddamip $\int a$ partium $t c=$ nutum. Cexterum fructus myrtis sdfimilatur.

H 3

Opos, 
If $\quad P A V L \quad A E G I N E T A$

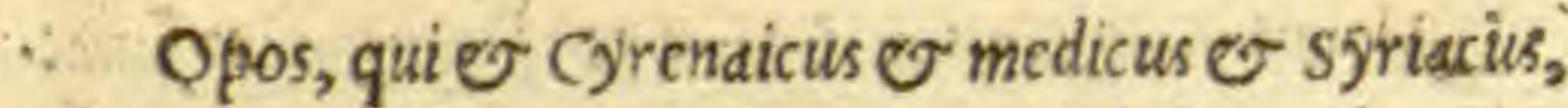
ficundum excellentiam dicuntur, calid funt uehemen= tere firttuofi.Maxtme uero cyrendicus, reliquisca - lidior, $\mathcal{O}$ partiom magis tenuum.

1

\section{Origanus.}

Origani, omnes fecandi $v$ attenuandi $v$ arefacien di, $O$ calefaciendi potentie funt int crtio ordtne.

\section{Oreofelimon.}

Oreofelinos, proximam apro facultatem habet, o fortaffs etiam uebementiorem.

\section{Orobanche.}

1.. Orobanche, calidi $\odot$ ficcieft temperanent, in pri mo ordine.

\section{Orobos.}

- Orobus, calefucti in primo gradli, arefacit uero in ficundo, $\odot$ quatenus quidem amaritudinem babet, ed $=$ tenus incidit $\mathcal{O}$ abftergut, atq; ab opilatione dimonet, Plusautem a frampts, fangutnem per urinam agit.

\section{Orminon,}

Orminon, \&qualceft marubioincaliditate, fedme= diocriter ficcum 0 abftergens. Venerem igitur exti $=$ -mulat. Purgat etiam cum melle oculorum albugines $\mathrm{C}$ oedemats difcutt, $\sigma$ furculos extrahit. Verum agrefte hortenfi multo ustidius eft.

\section{Oriza,}

- Orizd, quiddambsbet dcerbefcens, propter quod wentremmediocriter colibet. 
Orchis berba, hocatur alo nomine cyms orchis. bulbofam $\mathcal{O}$ duplicérad cemhabens. Ca'cfact $\mathcal{O}$ hus metfat, fed non admodum, unde Venercm fuccendit.

\section{Orchis minor.}

Orchis minor, calefact $\mathcal{O}$ ficcat, priort ex diam:es trorcpugnans, or cotum reprimens.

\section{Orchis ferapia.}

Orchis, quem $\mathcal{O}$ ferapida nominant, alij uero Tri= orchim, priori magis exiccat, oedemataigutur diftrd= bit, hulceráf; fordida $\mathcal{O}$ in putrediněucrgentra repur gat. Quanğ O Herpctes quog; fanat. Siccata tamen ue bementius aref acut, $u$ 'q; adeo propter ad/trictoriä usm, gua fit, ut cum utro pota, uenircm rets cat.

\section{Ofiris.}

Ofiris, exqua frunt coremata, fiue Cacorematd, amara, $\mathcal{V}$ id amoucndam opilationcm idoned. Quare Hepatis prefarctus apert?.

\section{Offa bumana.}

Off combtifta admodum digerentis o exiceantis funt potentue, mixime uero, u quibufdam placet, bus mana.

\section{Offa fum.}

Talum fuslum iftum, inflitiones $\theta$ tormina f $d z$ nat:Et quidam offacombufta dedut bibere cpicefium= $\dot{q} \mathcal{E}$ arthritim boc medt amine curautt.

\section{Talum Bubulum.}

H 4 Tdlum 


\section{P A V L. AE GINETA}

Talum Bubalum uftum, commotos dentes, diunt conftabilire, cum melle uere potum, rotundos lumbriz

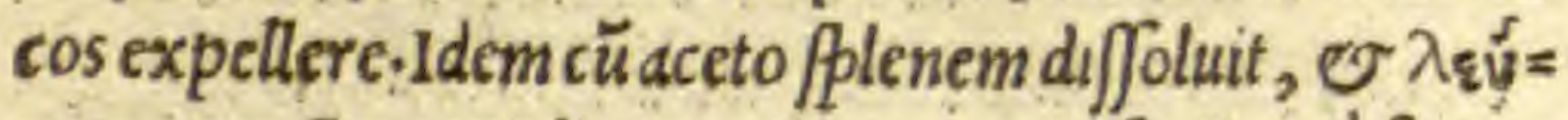
rog sinunctum medicatur, venercum eft. Porró femos ra bouls ufta at f; fubinde pota, bemorragias er relle quas fluxiones fiftunt.

\section{Teftę Oftraca.}

Oftracd, que ex fornacibus sunt, ficcantis $v \cdot a b=$ ftergentis funt potetula $Q$ Quare dentes abftergüt, $\mathcal{O}$ illi ta cum dceto prurigines, $\mathcal{F}$ exanthemata, $\mathcal{}$ podagri cos iunant cumcerato, etiam cboeradas difcuttunt. -

\section{Oftrea.}

Oftreorumte fta ufta, fimilis eft facultatis buccino= rumteftss. Etiam fiminus partium craßsiorü. Itaq; que infacie funt abftergit, e dentes albıficat. Eadem elota, mordicationem amitttt, humida $q_{\text {; }}$ hulcera incarnat,,adcicalricem perducit.

\section{Vrina humana.}

Vrind, omnis calida eft $\sigma$ acris potentie, at urind bominis, omnium propè aliarum eft imbecillima, exce= ptis porcis qubbus erecti funtteftes, uehemëter ad/trin git. Q Quidă inf fame cam bibētes feruatifunt.Lepras o bulcera, fordibus or impuritate fcatentia, achores 0 . furfures, abftergut. Preterea pedum attritus, fed néns dum inflammatos.

\section{Vrina pueri impubis.}

Ex bomine omnium efficacis $\}_{t m a}$, fft urind impubis pueri. 


\section{Vrina Muli.}

Mulorum lotium, articulorum morbis congruit:

Vrina Caprarum \& Camelorum.

Vrind caprarum, uentrem fubducit, o bydropicis bibendum datur.

\section{DE HIS Q VAE INCI piuntablitera r. \\ Pancracion.}

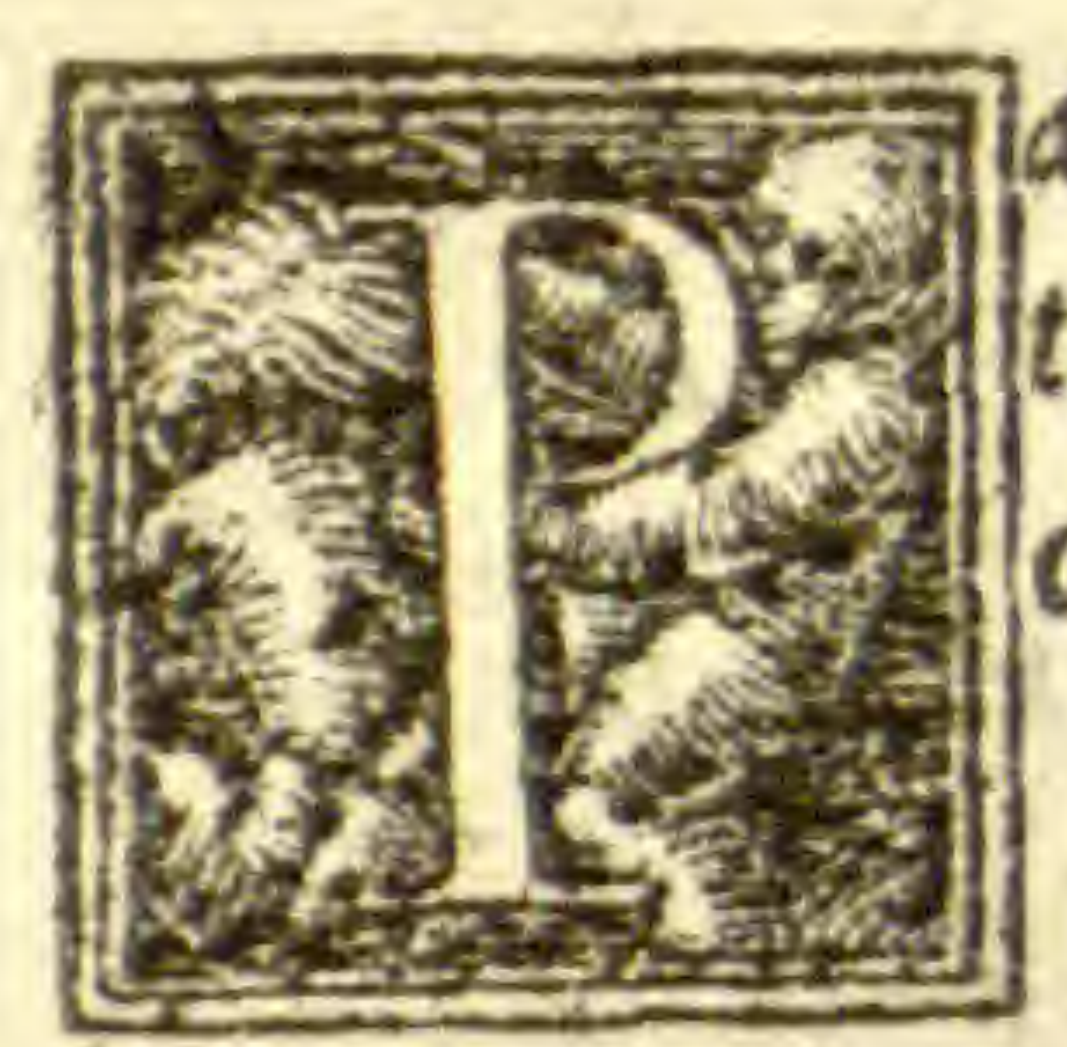

ancracion, Scille fimilitudinem $\sigma$ pos tentiam refert, pretcrquam quodimbe cillius eft.

\section{Paliurus.}

Paliuri folid er radix adftringunt, proinde tentris fluxum cobibent, e phymats non ad $=$ modumcalentia digerunt. Coterum fructus incidit in tantum, ut calculos ue ice comminuat, $\mathcal{O}$ thoracis $\mathrm{ex}$ : creationibus auxilictur.

\section{Panacos.}

Panaces Heracleon, ex quo exprimitur, quod uocds tur opoponax, tertij cale facientium ordinis eft, are faci $=$ entium uero fecund, ex corum numero que permulcent

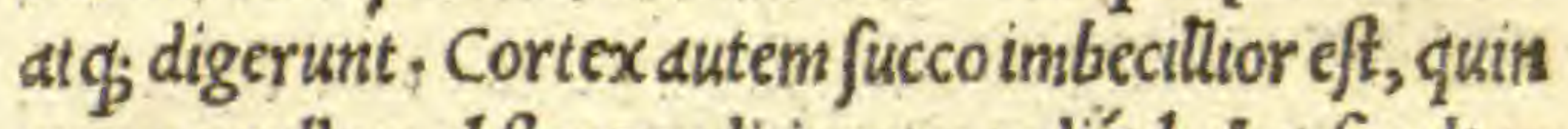
or nonnullam abftcrgendi, incarnandi $q_{\text {; }}$ habet faculta $=$ tem. Fructus men/trua a git. Panaces, aftlepium, of chi ronium, heracliocalidiord funt, ev ob id floribus uns cum fructu ad maligna hulcera, of phymats utuntur.

Papyrus.

H, Papyrus. 


\section{P A V L A $E$ G INE T A}

Papyrus, inufta qudem materia quedameft, uerum af ta cxiccat non aluter, quadm charta combufta . Cinists men pajyrumbectlior est.

\section{Paronychia.}

Paronycbia, ab ffectu fic nominatarft, quia para = rochias fanat. Vis elus tenuum partlum eff, $\sigma$ exiccss toria, er morfucarens.

\section{Pentaphyllos.}

Pentaphyllos, arefact in tercia diftantia, minime tas men acuita eft, idco ualde utilis perhibetur.

\section{Peplos.}

Peplos, quidam aly mecon a aphrode. Et hic frutex frecum, foluendi ui, tubumilis fimstem habet.

\section{Peplion.}

Peplium, e hoc quoq; fuccum babet, nafciturǵ; ad mare copiofum, catera inutle Semenhabet frruens $f=$ militer fcminipepli purgans.

\section{Piper.}

Piperisradix, cofto /imilem qualitatem habet. Por= rô fructus nuper quidem admodum germinantis, long $\ddot{u}$ piper eff. Temperamento caldum $\mathcal{E}$ humidum, uelut omphax.

\section{Piper album.}

Album piper, nigrodrr:us eft nam illud iam quafi fuper affatum eft, $\mathcal{O}$ fuper incođInm. Allogu utrung; tum cxid'facit, tum deficcat:

\section{Pepon.}




\section{DE SIM P ISICIE $\nabla$ S:}

Pcpon, abfergentis eft potentic, quare caro cius cphelides ${ }^{O} \sigma$ uitiligines extertt. Semé obturatisrenibus utile eft.

\section{Periclimenon.}

Periclimena folia o fruetus, inctdendi fimul $\mathcal{O} \mathrm{cd}=$ la faciendi funt potentie, adeo ut quadraginta dicbus po ta, urisam fanguinolentam cxigant, at q; excernant. Iu uant 0 lienof os, $\mathcal{O}$ difficulter pirantes

Periftereon.

Periftercon, in tantū extccat at nera gluttnet, $\mathcal{F}$ hemorrhagiam compe fcat.

\section{Petafitis.}

Petafitis, extertio eft ordine exiccantium, quare ad cacoethe $\mathcal{O}$ phagedena bulccra ed utuntur.

\section{Petrofelinon.}

Pctrofclini femen, calidum o ficcumeft in tertio or dine, $\odot$ fecandi uim babet. Menftrus igitur cit, $\odot$ uri

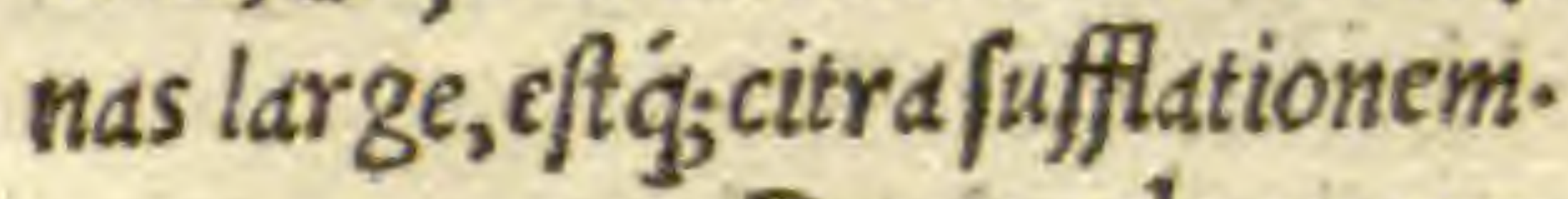

\section{Peucedanum.}

Peuced snifucco, $\mathcal{v}$ iure, $\mathcal{v}$ liquoreuchementer cd tefacienti, $\mathcal{O}$ digcrenti, $\mathcal{O}$ attenuanti utimur. Siquidè $\mathcal{\sigma}$ olfactu, $\mathcal{V}$ in potione fumptum, craßis, $u i f c o / i s q_{;}$in pulmone $\sigma$ thorace paßionibus medetur. Adhec $v$ induratos lenencs unat. Catuis dentibus inätunextëplo dolores fedde-Sed o neruorü paßtonibus congruit.Ra dix oßis fquamas demouet, $\mathcal{O}$ bulcera fandt, que cacoe the uocantur ${ }_{0}$ calida of ficca, fecundü tertü ordinem.

Piced 


\section{P A V L. AE G I NETA \\ Picea.}

Peuce, proximam pino facultatem babet, licet tem = peratiorem.

\section{Peganon, Ruta.}

Riuta agreftis, in quarto cft ordine calefacientium. er arefacientium - Hortenfis uero in tertio. Vnde etiä fecat $\odot$ diffrahitcraffas $\mathcal{O}$ lentes humores, per $q_{\text {; }}$ uri $=$ nam ducit.Partiumeft tenuium, $\sigma$ fine flatu, $\sigma$ ob hoc Venerem coercet.

\section{Piffa,Pix.}

Pix ficca calefacit in fecunddria diftantia, minus ta menexcalfacit - Ex contrario humidd, uires babet abfer gendl, concoquendi, $v$ digerendi, quiddam ctiam acer bum. Proinde $\mathcal{O}$ leprofos ungues eximit, lichenas de $\Rightarrow$ tergit, $\odot$ tumores concoquit, ad omnta ualentior, unde. aftbmaticos, e empyicos cumeclegmatc iuuat . Arida boc habet, quodad uulnera glutunanda magis congruit.

\section{Piffelron,}

Piffelcon, ex bumida fit pice, fed effentie tenuioris. Piftacia.

Piftaciorum fructuscommeftus, euftomachus eft me diocriter, $v$ hepatis obftructiones laxat, prectpue ad id poteff fumi, e odorarieorum decoctum, Contra fer pentium etiam morfus euxilio funt.

\section{Pitydes.}

Pityides, fructus picearum, uel pinei mall, mixte eff potëtie, partim fcilicet adftringentes, partim acres, 


\section{DESIM PLICIBVS.}

unde ex thorace conferunt fputamentis.

\section{Pityocampx.}

Pityocampe, eandem quam Cantharides or bu= preftes facultatem habent.

\section{Picex cortex.}

Picce cortex, dominantem habet acerbefsentem po tentiam, eoufq; ut inter trigenes $\mathcal{O}$ ambufta ad cteatri ccm perfanet. Ventrem etiam rctinet potus, o uulne= raconglutinat.

\section{Pityufa.}

Pityufa, $\mathcal{V}$ banc quidam fpecicm tithymalli faciunt; quod fuccü habeat ut ille, quod f; fimilter illis purget. Platanus.

Platanus, bumidioris $\mathrm{e} / \mathrm{t}$, $\mathcal{F}$ frigidioris effentie, unde folia uiridia, phlegmonas nafcentes adiunant. Cortex au tcm $\sigma$ pilule aridioris funt potef tatis. Vnde in aceto $c 0$ cta, addentium dolores fedandos conueniunt. Cum pin guedenc ambufta, adcicatricēperdsicunt. Cortexuftus, aridior $\sigma$ adffringentior fit, , adco ut lepras $\sigma$ bumis ditates hulcerumextergeat.

\section{Pulmo Agninus \& Suillus.}

Pulmo agninus $\mathcal{O}$ futlus, thi crtrigenes à calciamen tiscurare creditur.

\section{Pulmo Vulpis.}

Vulpinus pulmo reficcatus, afthinaticos iuudt.

Pulmo marinus.

Pulmo marinus, podagris pernionibuscömode illini tur.

Polcmomum 
PA Y $L$ AE G INETA

\section{Polemonium.}

Polcmonium, alij Phtleteriü, tenuium partium est, - ficcum, quare nonnullic exhsbent if chiradicis, $\sigma$ tor mino $i s$, e durum $/$ plenembabentibus, bibendam radi cemipfius cum uino:

\section{Polium:}

Polium, quod amarius eft, cuius etiam ad antidota uf ise eft, tertie eff calefacientium of ficcantium ordinis. Amouctigituropilationes ab ommibus intefturs, $\sigma$ wri nis, o menffruacit. Viride preterea pregrandia unt ver a glutinat. Siccatum, bilceribus uciofa confuein= dine medelur.

\section{Poligalon.}

Poligalon, ,uffera mediocriter foliababct, que po= ta, lic gignere undentur, manufefte calide 0 bumides potentie.

\section{Polygomaton.}

Po'ygonaton, m:xieeft fácultatis. Siquidem conftat exadftrctione, acrimonia, of amaritudine quadam, quafi faftediofa, propter quod ad multum accommodda tur. Radice cius quidam procataplafmatcutuntur, or ex facie neuos illo extergunt.

\section{Polygonon.}

Polygonon, babet qutddam acerbefcens, fed predos minatur. Eft aquofum frigidum in fecundoordine, 1 u= uat catapla fmatcilltum, cos, quibus ftomachus ardet, Orercfipilata, $\sigma$ anlidas phlegmonas, ơ fi quide eft aliud ctws. 
aliud cus generis morborum - Et tale cum fit, fluxianes suna. A pparet ctiam nonnibil ficcare, o mas ad om = na foemina efficacior eft.

\section{Polyciemon.}

Polycnemon, cale fact o ficcat in fecundo ordine, itaq; unlnera conglutina.

\section{Polypodion.}

Polypodium, ficce usbementer, of fine morfu eft potentie, e potum per inferna purgat.

Pom pholix.

Pompholix, exiccat, fine mor $f u_{2}$ ex bis, que fordis $d a, \mathcal{V}$ alta malc habitudenis bulcera incarnant - Milccs= tur o ophibalmecis ficul atibus.

\section{Purpurx.}

Purpure ufte, etufdem funt potentice cum of tracis. Eorum opercula cocla inoleo, fluencs capillos retmét. Porrô cum acetolienes tumenies contrabunt. Locorum ctuam prefocatione concidentes foemunas cxcutant, $\sigma d$ partufecundas eigciunt.

Potamogiton.

Potamogiton, acerbefu o refrigerat, modo quo o polygonon, aqualis per omnia cum co potentie.

\section{Poterrhion.}

Poterrbion, alij Phroenum, neruos precifos, o re reliquas neruorum affectiones fanat, of unlnera glu= tinat.

\section{Prafum ${ }_{2}$ Marrubium.}

Matrubiuns 


\section{P A V L. AE G I N E T A}

Marrubium, caleficit in fecundoordine, fed magis exiccat.P roinde aperit opilationes hepatis, $\odot$ fplenis, o que funt circa thoracem, $\sigma$ pulmoné, $\sigma$ menftrus aduertit. Atcataplafmate impo fitum, abftergit, $\odot$ diz gerit, uel ut alijs placet, abftergit er incidit:

\section{Prafon, Portum.}

Porrum, mali fuccicft, acutã uim babet, ut inquit Di

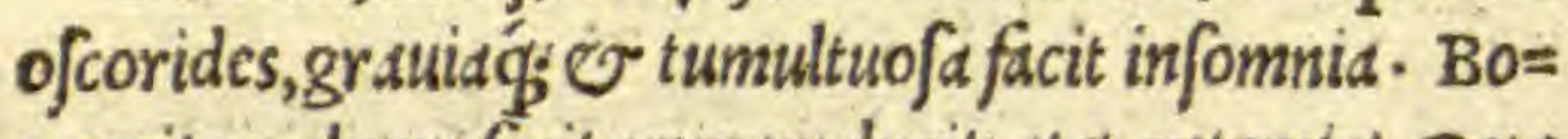
nam item aluum facit, urinam ducit, at $q_{\text {; }}$ cxtentat. $\mathrm{O}$ ut damtenus etiam abftergit, unde cum prifand coctum, pe Eforis excreationes adiuuat. Porrô femen nephreticis mifcetur facultatibus. Et folia ip fa ad/tringunt, quams ob cauffam fanguinem fiftiteorum expreffus fuccus.

\section{Pinus.}

Pinus, fimilem facultatam habet cü quercu, al iquan totamenillaulentior.

\section{Propolis.}

Propolis, cale facit quidem in fecundo ordine, fed ats ftergitmediocriter. Partium tenuium, $\widetilde{C}$ ab obftructio nelibcrande potentia.

\section{Ptarmice,}

Ptarmice foliafternutamentamouent, e totus fru $\Rightarrow$ tex, quia fubamarus eff. Reliquas echimos feis digerit. Eft autem calida o ficcs, uirens quidem in fecundo ors dinc,arefactaintertio.

\section{Ptelea,Vlmus.}

Pteled, arefacientis eft potentie, or abftergentis. Qusmobrem 


\section{DE SIM P L I C I B V S.}

Qumobrem uninera glutinat. Cortex uero multo ma* gis, firccens, uncultuce circumligetur - Cum acèto lepras quo $q_{5}$ fanat, radices ciufdem funt poteftatis. Nä er dccocto carum quidam perfundunt fracturas que calloopushabent:

\section{Pteris.}

Pteris, ficce potentix of oo dimare, quare embrya - latos lumbricos occidit, quatuor drachmarum pone derecum melicrato, quia dutem 0 medtocriter quoqs adffringit, fit ut hulcera fine mordicatione exiccet.

\section{Picnocomon.}

Picnocomont, digcrentis er dcuta potếtie eft, ctsius folia phymata, $\mathcal{O}$ furunculos difijcunt. Fructus ad eda dem udlent, e infixa ad extimam fuperficiem trabüt; radix bulem flauam expurgat:

\section{Pyrethrum.}

Pyrethriradix, caufticambabet facultatem, of den tiwis reffigeratortum dolores mitigat, aduer fum q́; rigo res, dnte periodum conteritur cum oleo, ad hacftupiz dos quog; erefolutos ad iunat.

Pyros, Triticum.

Triticum, calef acit in promio ordine, mediat'; inter

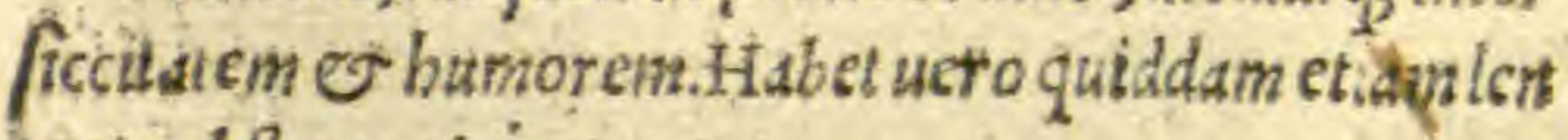
poris obftruentis q; nature.

\section{Pitya Coagulum.}

Coagulum, unuter fum acute of fubtiliat is er dis gerentis, of poftremo maneffte etism arefacientis ef potentic.

1 Coagulum 


\section{Coagulum Leporis.}

Leporum cosgulum, cptlepticos tunare, fi cum aceto bibatur, $\odot$ proflunium fanare muliebriproditum eft. Sed $\sigma$ lac in uentre codgulatum diffoluere, o fangui nis exftreationes cobibere pradicant.

\section{Goagulum Equinum.}

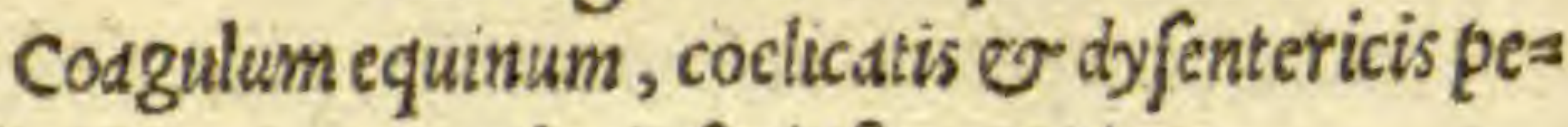
cultarter accommodari, fcripfere quidam.

\section{Coagulum phoce.}

Codgulum phoce, eafdem cum caftoriouires habete

$$
\begin{gathered}
\text { DE H I I S Q } V \text { AE I N= } \\
\text { cipiuntablitera } R \text {. }
\end{gathered}
$$

\section{Racos pannus.}

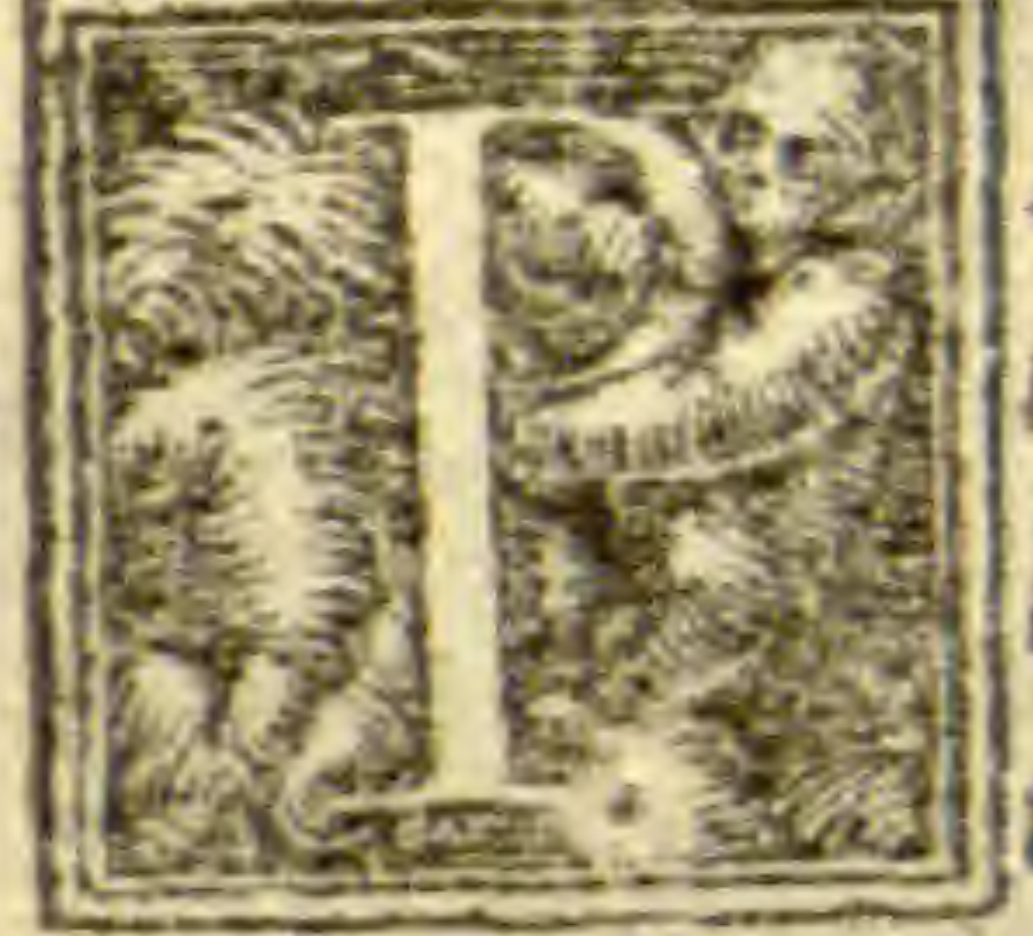

Annus ufturquilibet, lane ufte ex qua texitur, facultatem babe - Linum com bufium partium tentuam fumumex $f e$ mittit, qui fuffitum, catdrrbosaperit,

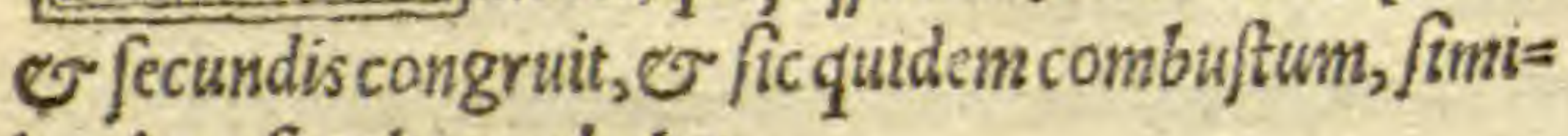
lem lana facultatembabet.

\section{Rhamus.}

Rhamus, exiccat quidem in fecundoordine, refrige rat ucro in primo. Qutmobrcherpctas fand, E Ere fipclatd, calid alliem non ualde. Ceterum ad hac fo= lijs utcndum.

\section{Raphanos.}

Raphanos, calcfuct mtertia diffantid, at $q_{\text {; }}$ arefacit in fecundd. Ambarum femen webemens eft, digeritóp figillatd, 


\section{E S I M P I C I B V S:}

58

fugillata, 0 alia id genusliuentis.

\section{Reon, Ra.}

Reon uel Ra, compofitumefl, exterreftrifrigidd

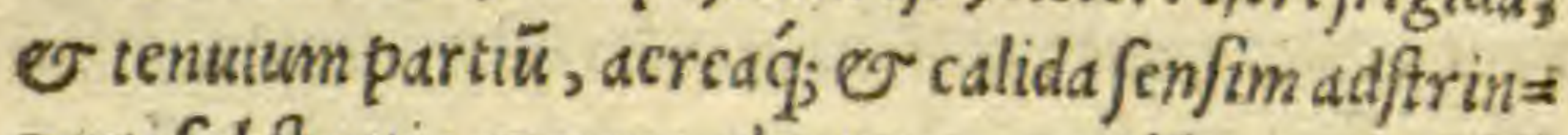
gensifubftantia Quaread rupta, conuulf $a$, o ortho= pnocam cöducit, liwores difcutit - Propter ddftrictionë uero, Hemoptoicos, coeltacos, $\mathcal{O}$ dy fentcricos iuudto Ratione quoq; ustiu $f q$; facultatis, ctiäbepaticis cöferts Rhefinæ.

Rhefine omnis calefaciunt $\mathcal{O}$ ficcant

Rhefina, Terebinthina.

Rhefind, Terebinthina, precateris merto topridres partes meretur, quoniam digerit, mollit, e partium te nuum cũ fit, etidm abffergut, ac proinde, p poras fanat :

Rhefina, Pinea \& Strobilina.

Pinca of frobilind, quaddmtenus acrior rbefine, non tamen iccurco magis digerunt uel aftrabunto

Picea \& Abietena.

Medis inter has funt pices $O$ abictina.

Rofa.

Rofe, uis eft compofite effentie, exaquofa calids, adftringent, acerbcfcenti $\mathcal{O}$ amara faculiatibis. Flos tameit ear um adhuc magis acerbefcit rofis, or ob hoc srefacts.

\section{Radix Rhodia.}

Radix Rodia, calfact is fecundoordine, partium tenuium, 0 digerentis potentis.

$I \geq$ Mals 


\section{B. P A L. AEG INETA \\ Mala Punica.}

Mala Punica, uel Granata, dulcia, in uniuerfum quis dem acerbefcentis potentie funt. Qu smobremreffige rant o exiccant, auffera magis refrigerant, ficciors acuta funt $\odot$ ftomacho commodiora, acida refrigc= rant, $\sigma$ cumboc amplus quiddam habent quo inci= dunt - Porró ficci ipfius acimt, acerbiores funt fucco, neenonaridoores. Et hijs ommbus $\lambda$ É $\mu \mu \alpha \tau \alpha \sigma^{\prime}$ id'a, ideft , putamina ipfa, qrae fic uocant - Parem um ba= bent 20 Cytini,fic enim illorum flores appellantur.

\section{Rhus.}

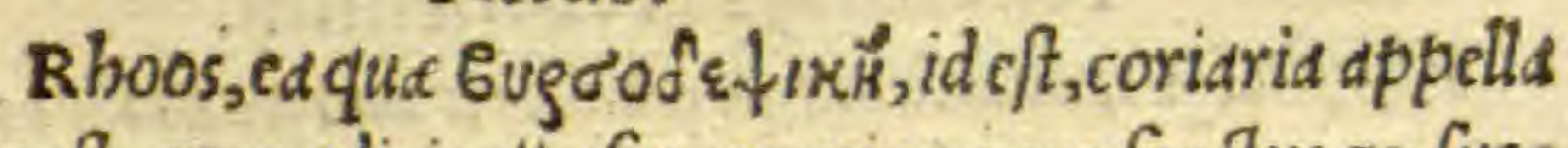
$t a \in f t$, $\odot$ mediciseft $u f$ fut, maxime iero fructus $\odot$ fuc $=$ cus, uebemrnter abftergentis $\sigma$ ficcieft temperamen= ti, extertio ordene, abftergentis q́; in fecundo.

\section{Rhypos Sordes.}

Sordes, ab ere ftatuarum, uel ab uafis in quibus " $\alpha$ :

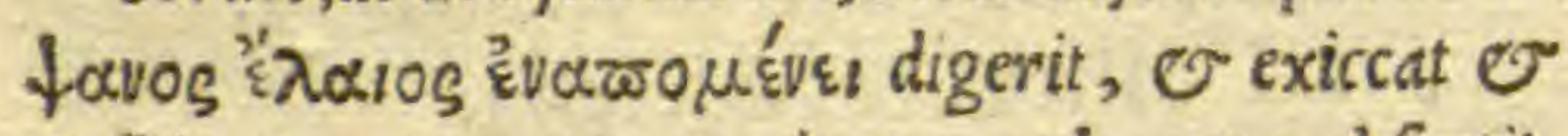
mollticat mediocriter, cruda igitur phymata, defcutit.

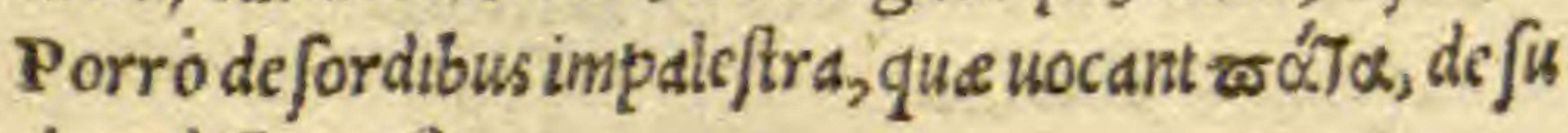
doredictum ejt.

\section{Sordes Aurium.}

- Sordesque in auribuscontrabuntur, paronychias fantare dicuntur.

\section{DE HIIS Q V AE I N= cipiuntablitera S. Sagapenum.}




\section{DE SIMPLICIBVS.}

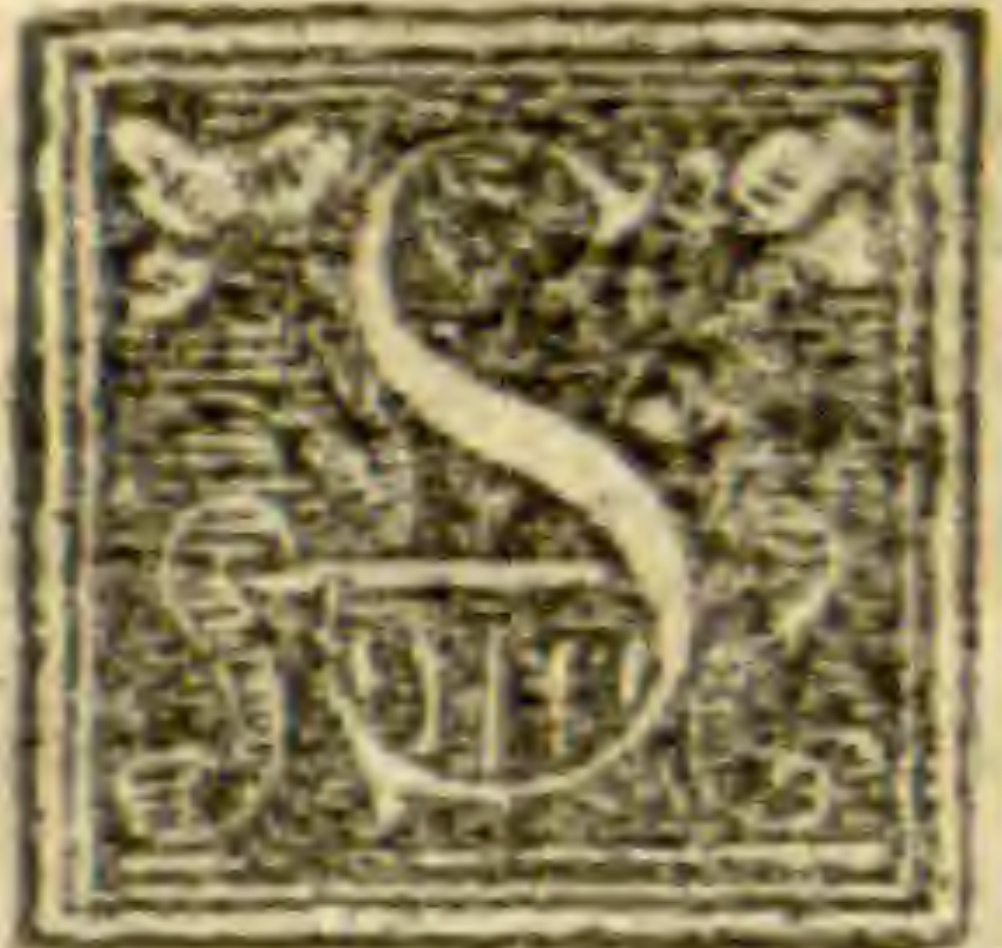

A gapenum, liquor eft frolale, calidus, cum partiwm tenultate, $\mathcal{O}$ quodamte nivs abftergens. Proinde oculorumicied rrcibus o fuffufionibus congruit.

\section{Salamandia.}

Cremate falamandre, cinerem aliqui mifcent, fos pticis o lepricis,p foricis'qf facultatibus.

Samfuchus.

Samfuchus, cale facit of ficcat in tertia diftätia, ef $\dot{q}_{\text {; }}$ partium tenuium, $\mathcal{O}$ arefacientis potentie.

Sandaraca. nicums.

Sandarace, cauftce eft potentia, perindc ut Arfe=

\section{Sandix,}

Sandix, expartibus conftat tenubus, o temper $1=$ mento ficco, non tamen excalefacienti.

\section{Saprotis, Caries lignorum.}

Carles lignorü, ex maxime que exulmoeff, mundifi sat $\mathcal{E}$ incarnat bumida hulcera.

\section{Sapon,Sapo.}

Sapon,abftergentis eft potenice.

\section{Sarcocolla.}

Sarcocolla, lachryma eft Perfice arboris, emplaftic cd; $\mathcal{O}$ fine mor $u$ arcfaciens, quam ob caufam uulncrs conglutinat.

Sarxiphragos, Saxifraga.

$$
1 \text { 3. Saxifragd, }
$$




\section{P A.V. L: AE G I N E.T.A}

Saxifragd, urtuds cit, or uefice calculos confringite Satyrion.

Satyrion uel Triphyilon, bumidum eft $\mathcal{O}$ calldum temperamento, of flitus ciens, quare uenerem exufcl= tat, ferunt quidam $\mathcal{E}$ opit thotono medert, $c \bar{u}$ usno aus feropotum, Certe Galcrus in librocui ttulüfecit, De Theridcis.Triphyllum, inquit, byacintho ab aëre tur= get, o femenagrefit Garihamo habet fimule.

\section{Lacerta,}

De Lacerta fuo dictum cff loco. Preter hec tames

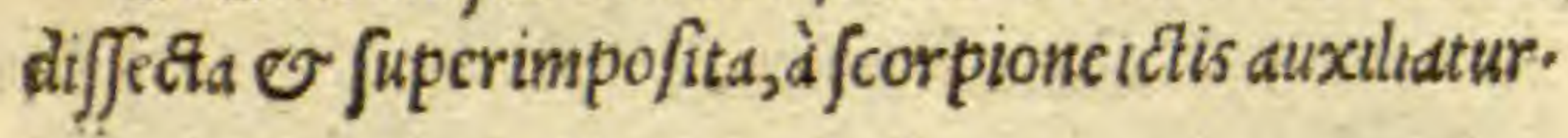

\section{Selinon Apium.}

Selinon, calidum eft, at $q$; adeo, ut urinds $0 \mathrm{M}$ enfes moucat . Flatus quoque difcutit, ov magis femenqudm berba:

\section{Sefeli.}

Sefelcos radix, evinprimis etiam fructus impenfe calefacit, cum partum quadam tenuitate. Ideo urinas ducit - Et aduer fum Eptlepflam, $\mathcal{O}$ orthopnoeds $r c=$ mediocf.

\section{Sepix Tefta,}

Sepie Tefts proxima eft oftreo potentid, fed partium magis tenuium o abfergenuor. Q $u \Delta$ mob $=$. romufta, O cum fale foßsli mifta, oculorum pterygia. extenuat - Inufta or conficatẹ pculorym oukw Grig, 
D E SIMPLICIBVS.

o que circa reliquan cutem funt aperitates cmun. det dt $q_{j}$ abftergit*

\section{Sefamoides.}

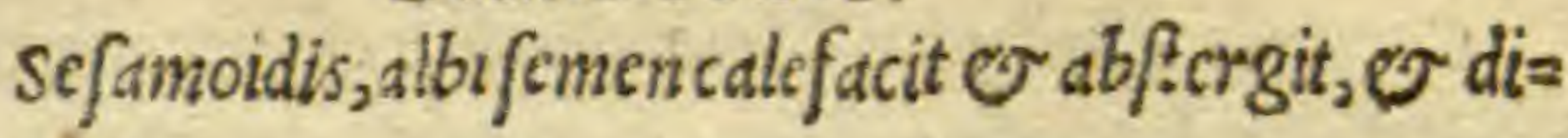
rumptt.

\section{Sefamum.}

Sefamum, non paulum babet lentoris atg; pingue=.

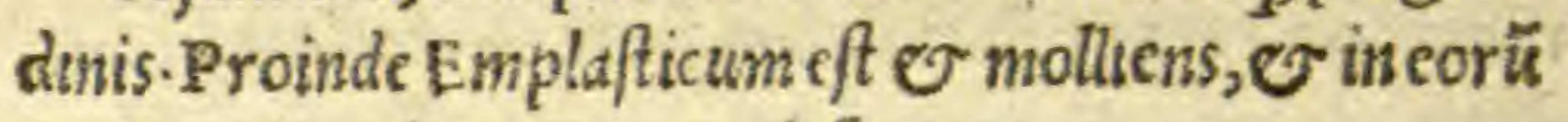
cenfu, qui mediocriter excalefacunt.

\section{Seps.}

Seps, quàm alif cha!cidicam lacertam uocant, in uis nopota, ab feipfa mor fos perfanat.

\section{Sialon, Saliua.}

Saliud, bominum ieiunantum $\mathrm{cx}$ impenfe concos quentibus $\mathbb{\sigma}$ abftergentibus cft, llita gitur puerorum lichenas fanat, ov tumores digeritcü tritico, quod pri= usin ore fit comman fum, deinde fupcrimpofitum, $\mathrm{H} y=$ popia delet, aduer fißstma beftys, hominé pertuétibus.

\section{Sideritis Heraclia.}

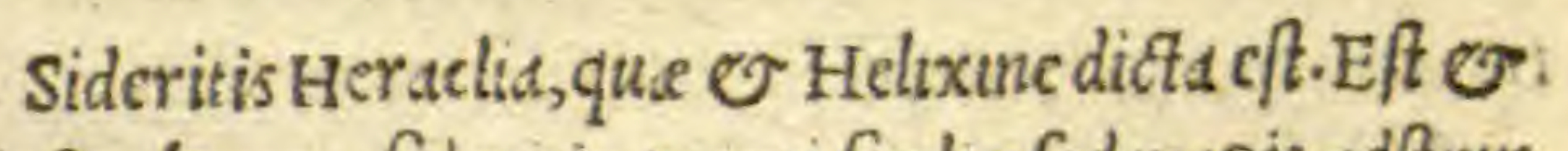

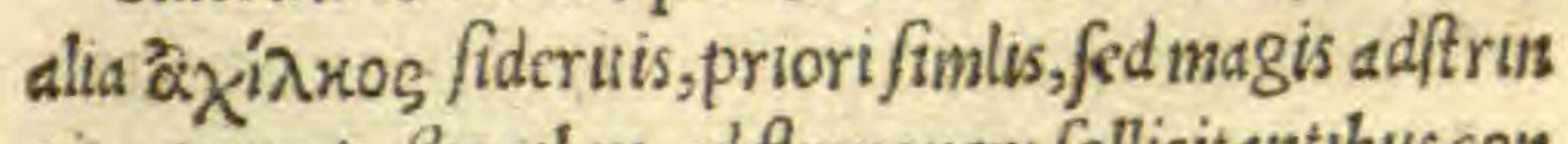
git, quare pa\}tonibus, ad fluxionem follicitantibus con gruit.

\section{Sidetos, ferrum.}

Ferreum in aqqua alguotes exinctüuebementcr fic calidi potcntiamundui Hecugitur aqua pota fileni un= I 4 durato 


\section{P A V L AE EI NET A}

dur ato inprimis conuenit. Si uero in uino extingur fues, rit coeliacis e dy fentericis confert:

\section{Sycios Cucumis.}

Cucumis, af culcntus fuburidis eft, refrigerat qfor humectat in fecundo ordine. Quare er caro quidem. sius coeta, lent ph́legmonas, femen urens ac recens abs fitrgentis 0 inciforia eft potétie, Proinde urinas pro mouet $\sigma$ corpus flendidum fact, arefaclum ficcat; in primo, $\sigma$ ualeter extergit, idem preftat o radis.

Cucumis agreftis, Elaterium.

Exagreffis cucumeris fruedu expreffus fuccus, Ela terium uocatur, folia or radixlccet fimlem uim babes ant Elaterio, tamen fint imbecilliord, radix ab/fergit, digerit o permollt - Succus exuccat:

\section{Sillybon.}

Sillybon, pinofa cft planta, albochamelconti nŏ abs : fimilis, efculenta, cuius radicis fuccus potus cum mez: kcrato, ponderc drachma unius, womitum mouet, s

\section{Silurus,}

Silurus, muriacöditus at q; cömeftushumidam are= teriam repurgat, cataplafmatus, infixa extrabit.

Blattæ qua in piftrinis inueniuntue"

Blatta, que in piftrinis inucnitur, concifa atq; de= socta, durium dolores fanat,

\section{Silphium.}

Silphium, calefacit Itrenue. Ef autem fatuofum, dc proinde coctu difficle, dlioqui tamen extra corpus officatius, 
effrcacius, ev omnum potißumum liquor, admodum tra hentis potcrtle. Habct quiddam ettam excrefcentias amolicnd:.

\section{Sifon.}

Sifon, calidus $\mathrm{E}$ concoctiuus eft, adeo, ut $\mathrm{v}$ urinas o Menfes moueat . Vifcerum quoque obftructiones apperit .

\section{Sifaron.}

sifariradix cocta. ftomacho falubris eft, fed ov uri= nas quoq; propeDit, calida iuxta fecundum ordenem, for ta 3 equia partium eft tenumum, 0 digerit facile. Semen tamen ualentius cft, propter quod tormino fis cum uino. datur.

\section{Sifymbrium,}

sifymbrium, quod o cardamomumuocant, propte red quod cardamo fimile eft. Stccum quidem calfacit $O$. arefacit or arefacit in primo ordine, Humidum uero m focundo.

\section{Scammonia.}

Scammonia, calide, $\mathcal{O}$ digerentis, $\mathcal{O}$ detergentis biliofor rum humorum potentic eft.Tum maxime fuccus, gui phymata difictt, $\mathcal{O}$ embria occidit appofitus. Ade

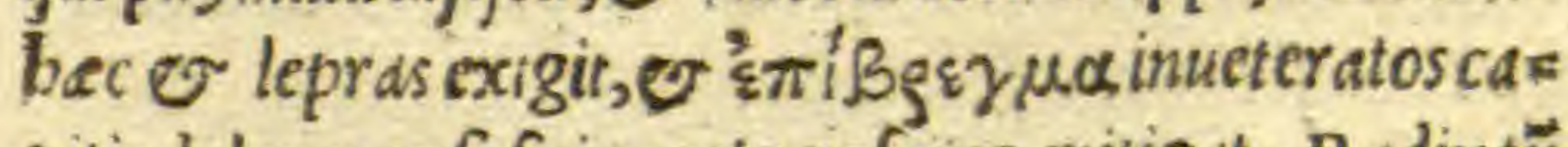
pitis dolores, $m$ fufain accto rofacio, mitigat - Radix $t \ddot{u}$ perfe ipfam, tumetiä decottd pota, aluum de ijcit - Cums. aceto $\mathcal{Q}$ bordeaced farina fric $a_{\text {, }}$ coxendicis doloribus: cmplaftrimodo imponitur.

$$
\text { I5 Scandix }
$$




\section{PA V L. AE GINET A}

\section{Scandix.}

Scandix, agreffecf t bolus, calidum $\mathcal{O}$ ficcum in $f e=$ cundo ordine, uires meiend l habet, $\mathcal{O}$ abinteftunis op= pilationes ammoucnds.

\section{Scincus,terreftris Crocodilus.}

Que foincirenibus adiacent, ad cupidinem inflam: mant, quam fubinde extinguit lactuce femen cum aque potum, e lentium decoctum cum melle.

\section{Scilla.}

Scilla, inciforie eft facultatis, calcfacitó; of ficcat. in fecundo gradu, praftat autem a fam aut coctam fus mere.

Scolopendra marina, uel Centipes.

scolopendra marina, coctatn oleo, $\boldsymbol{v}$ illita, pilofas corporis partes glabras facit. Eadem mambus tradd = ta,pruritum facit.

\section{Scolopendrion.}

Scolopendrion, ip fum eft Adplenion.

\section{Scolimon.}

Scolimon, calefactt $\sigma$ exiccat in fecundo ordine. Rd dix copiofam, eamí; male olentcm iriram eijcit, $f i$ quis coctam cum unno biberit-Axillarum quoq; $\mathcal{E}$ totius cor poris uirofos odores fanat.

\section{Scordion.}

Scordlon, $x x$ diuer fis conftat potentijs, fiquidé amas rume ff, $\mathcal{O}$ acerbum, $\mathcal{O}$ acre. Q $u a m o b r c m$ purgat, fis mulctiam calcfacti inteftund. Vrunds quoq; o Menfes. 
DE SIMPIICIBVS $\sigma_{2}$ cgit. Refrigerata omnia calefacit, pratered eo uulncra conglutinat, atg; do for dibus repurgat. Ad poftrernum ettam ad cicatriccm perducte, aridlif follicet impo fitum.

\section{Scorodon.}

Scorodon, calefácir $\odot$ ficest in quarto ordine. $C_{e}=$ tcrum opbiof corodon, id eft, agrefte allum, domeftico walentius eft.

\section{Scorodoprafon.}

Scorodoprafon, e guftu 0 potentia mixilum eft, aballioe prafodiuerfum.

\section{Scorpioides.}

Scorpioides, calefacit in tertio cxce $\iint u, \mathcal{v}$ arcfacit infecundo.

\section{Scorpius terreftris.}

Scorpius terreffris, crudus superimpofftus; fuijp $_{\text {is }}$ us plaga $\mathcal{E}$ iflus, remedium oft.

\section{Scoria.}

Scoria qualibet uehementer cxiccat, maxime uero Fervi,que decoetd, $v$ fubinde ad liworem redacta, $\int_{a=}$ nemftillantes aures, mirabiliter fanat. A vuxiltatur $\mathcal{E}$ bis gui acontum biberunt, cum oximelite pota.

\section{Scoria plumbi.}

Plübi fcoria, a ßlmilatur plübo, fcdmagis adffringit. Smilax.

Smilax, que o Cacios, arbor eft deleterie poten= Aise, id cft, uenerofe. 
- PAVL. AEGINETA

\section{Smyrua,Myrrha.}

Smyrna, fecundi eft or dinis calefacientium $\sigma$ drefa cientium, proinde catapla fmate impo/ita, capitis uulne ra glutinat. Habet quiddam etiam amarum, quo nimis rum uermes necat, $\mathrm{E}$ embria conficit - Quadamtenus ctiam abftergit, ophthalmicis facultatibus mifcetur. Et que circa thoracem funt, mundificat, ftne tamĕ arterles exaperstione.

\section{Smyrna,Beotia.}

Smyrna Beotia, uim habet calefaciendicalidam $\mathrm{mol}_{2}$ liendi $\sigma$ diffoluendt.

\section{Smyrnium.}

Smyrnium, quod aly Hippo felinon, alij selinon agre

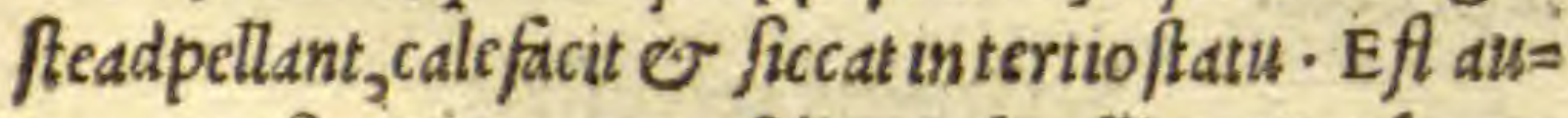
tem apto fortus, $\mathcal{O}$ petrofelino imbecillius, Menfes $\mathcal{O}$ urinas agit.

\section{Sonchus, Cucurbita fylueftris.}

Souchos, ficataplafma umponatur, manifeftarie ftrii gitatg; refrigerat - Arefactus mediocriter excalfacit.

\section{Sparganion.}

Sparganion, arefactentis e boc potcntic.

\section{Spartum, Se.}

Spartum ${ }_{2}$ quo untes adalligant - Huius fructus unà cum flore potus cum melicrato, obolorum quin $q_{\text {; }}$ men= furd, purgat per fuperna, ueluti belleborus albus, fine moleftid - Porrô fruetus fcorfum per inferna tanium. Virgule if chiddicas nicdicantur. 


\section{DE.SIM PIICIB VS}

\section{Spongos, Spongia.}

spongia recens, $\mathcal{O}$ que maris adbuc odoremreti= net, tmpof ita cum aqua, uel uino, ueloxicrato, uninera conglutinat. Vita autem, acute $\mathcal{\sigma}$ digerentis fit poten tie, ev cum apphalto, wel luquida pice exarefacta, $h_{e}=$ morrogias fifat.

\section{Spodion.}

- Spodion, siufdem facultatis eft cum pompholygi.

Staphilinos, Paftinaca.

Paftinacd $\mathcal{O}$ urinas mouet, $\mathcal{O}$ Menfes urget, poo tißimun femen er radix - Folia recentia, abftergentis potentiecum fint, phagedenicis hukeribus cum melle congruunt.

Paftinaca agreftis.Daucus.

- Paftinaca agreftis, ad omnta, domeftica nalentior. Stachis.

Stachts, marrubio fimilis, frutcx calefacit in tertio ordine, prounde Menfes exigit, embria interficit, or rijeit fecundas.

\section{Steata, Adipes.}

Adipes omnes bumet dant, of cale faciunt humanum corpus, idq'; fccundum temperamen: um animantum, exquibus defumptafunt potentia, uariant.

\section{Adeps fuillus.}

Adeps fuillus, on:nimm maxime bumcidet, potentid non admodüdiftans olco, Quamobrem morfus omnes retunditachebctat. 


\section{P A V L. AE G I N E T A}

\section{Adeps Caprarum.}

Adeps Caprenus ficcior eft, or citius denfatur, pro pter quod recle intefinit torminubus inijcitur:

\section{Adeps Anferinus $8 \&$ Gallinaceorú.}

Anferinus adc ps admodum calet, eft $q^{\prime}$ magis par= tium tenuium, pof thunc $\mathcal{F}$ gallinaceor $\tilde{u}, \mathcal{O}$ galinaru.

\section{Adeps Leonum.}

Omnium calidijstmus atq; fubstlisimus eff adeps les onum.

\section{Adeps Vrfinus.}

Vrfinus, capillorum defluzia explct.

\section{Adeps Vulpinus.}

Vulpinus aurium doloribus medetur.

$$
\text { Adeps Viperx. }
$$

vipersrum adipem ferunt, ubtradicitus pilos cuul= feris, probibereillos recrefcere, ad hebetes quoq; och= los efficacem cffe.

\section{Adeps mafculi pecoris.}

Ex predictis ommibus hoc habe, quod calidior $5 \mathrm{~cm}=$ per $\&$ ficcior cft mafculorum anmmalium adeps, of ho rumip for um exectorum minus, tum cald dus, tum ficcus

\section{Adepsuetus.}

Adepsinueteratus, quoduturus feruatur, hoc reds diturcalidior.

\section{Stimmi.}

Stummi, arefictt, $\mathbb{\sigma}$ nonnibiletidm adftringit, pro= inde ocularibus mifcetur collyrijs. 


\section{DE SIR P L I C I B V S: $\sigma 4$}

\section{Stcebe.}

Stcbes, fructus o folla ficcant in tertia dis d antia, for cultate adftringunt, nam uulnera pregrandia figunt. Decoctum quoq; corum addyfenterias 0 r bemorragi as compefcendas, item ad aures pus diftllantes datur: Deniq; expligdoculorum $\int$ uffifiones, cataplafinate im pofita imuat.

Stoechas.

Stoechas, abobfitructione liberat, attenuato; at $q_{;}$ adftringit - Inteftina omnid, o totum adcocorpufculu corroborat.

\section{Stratiotes.}

Stratiotes, ille qut in aquo is nafcitur, refrigerantis cf potentie. Terreftris nonmibil conftringit, quo fit, ut bulccraconglutinet-Sunt qui ad hamorragias quog; $u=$ tuntur, or ad figtulas.

\section{Strychnon Solanus.}

Strychnon, aliud quidem horten $\int c, \&$ efculentum, quod adffringit, or refinger at in fecundo ordme. Alitd quod dilcacabon uocatur, fimulc hortěfl, fructum ferens. qui urinas follicitat, uuarum acinis fimilem.

\section{Solanum fomnificum.}

somrifici Solan radicts cortex, in uino drachme ponderc potus, foporem inducit. Semen urinam cit, Tertij est ordinis, refrigerantium - Qu ure fi plus quä duodecim corymbi cius bibantur, furorem ficit.

\section{Solamum furiofum.}




\section{P A V L A A G T N E T A}

Qusrtum genus salami intra corpus fumptum, inus - tile acnoxtum, foris cataplafinate illitum, bulcera depd= feentiafanat, fécüdienreffrigerando o ficcädo ordinis Stypteria, Alumen.

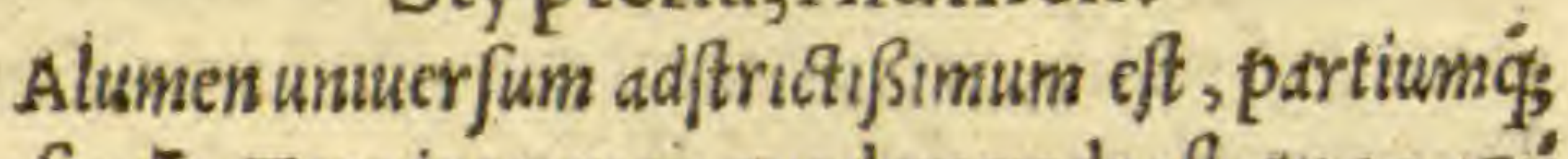
denf iorü . Tenuior tamen quodammodo eft, que gis 5 is dictseft, deindeftrongyle $v$ aftragalote - Supra modü

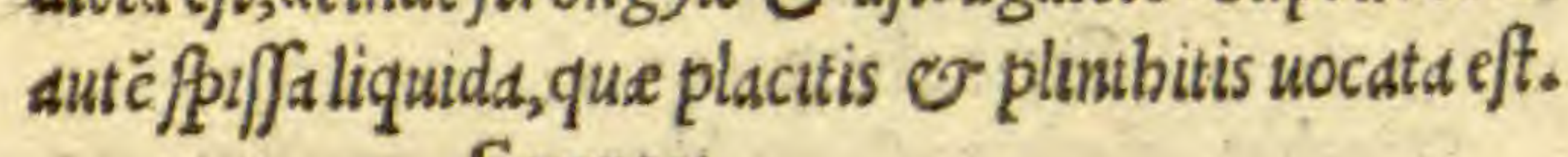

\section{Styrax.}

Styrax, calefacit, noolit, e concoquit. Proinde tufs fes, $\odot$ catarrhos, $\odot$ coryzas iturat, Menfes quo $q_{j}$ tum pota, tum adpofita aduertit.

\section{Syca, Ficus.}

Ficus sicce, callde funt potcntie in fecundo prope = modum ordine. Pinguiores, dura tubercula magis cori= coquunt. Que uero funt acriores, uel acerbiores, exier gunst ac digerunt. Decoctum corum melititißmus fit, confiftentia melli quid fimile. Efr etiannum, quod si= reon appellant,potentia fimile melli. Virides imbecilliz oris fintut fubftantie. Ambe tamen, id eff, tamficce, quä bunscefe, tuentrem fubducunt.

\section{Caprifici.}

Caprificorum Fici, acris o digerentis funt poten tie, pariter $\mathbb{E}$ domefticarum großi, quas ò $\lambda$ ivorses Grecinocant.

\section{Sicre ficus arbor.}

Ficus arbor, calids eft temperamcnti partium q́; tes 


\section{E S I M P L I C I B V S. $\sigma_{S}$
m, ueluti diuirarelicet éfucco, folijs e liquore,}

tentuitum, ueluti diuinare licet é fucco, folijs ev liquore,

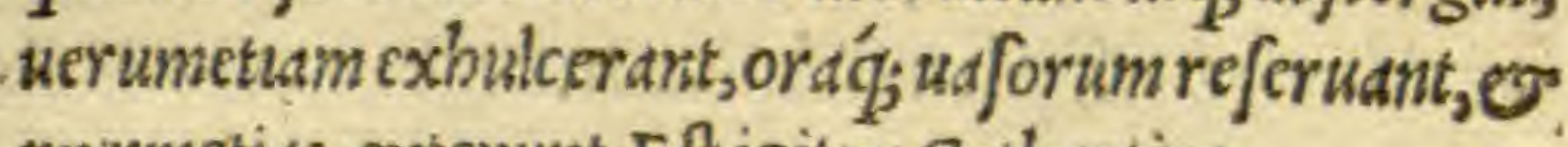
myrmetias exterunt. Eft igitur Catharticas.

\section{Ficus agreftis.}

Agreftis ficus, quam caprificum nominät, ad onnia domeftica efiulentior.

\section{Sycomorus.}

Sycomorifructus, male nutrit, o ftomacho infeftus eft. Catcrum fuccus arboris facultatembabet mollien= di, uulnera glutinandi, $\mathcal{O}$ tumores difijciendi . Et bibi= tur $v$ inungitur aduer fum ferpentum morfus:Dura: toslienes, ftomachidolores, E inacceßionibus febri wirhorrores.

\section{Symphython,}

- Symphytam illud, quod petreumuocant, excontre trarijs confiffit potentijis Hobet namq, uim deficcandi, qud nimirü in thorace etrenibus pus expurgat - Ouan dam etiam contrahendi, qua hemoptoicos coniul $/ \mathrm{f}, \mathrm{rd}$ pta, fuxciones rubras o enterocolas iuudt - Et fuper hec omnia, habet quandam calidam bumiditatem, qua fitim extinguit 0 arterie dfperitates.

\section{De Symphyto maioti.}




\section{PAV L AEGINETA}

symphytum maius, uifcofum, figlte fimile eft, utun tur co ad omnid, ad que iam dicla.

\section{Sphondylion.}

sphondylij fructus o radix acute funt $v$ incifo= vie potentie. Lia q affinaticis of Epilepticis, \& ictes riciscongruunt - Porró radixcircumrafa atgimpo $i=$ ta fiftularum callos crodit, floris etiam fuccus inueters tishuiceribus auxiliatur.

\section{Schinos Lentifcus.}

I entifcus, fccundum omnes partes eilus exiccat in fecundoordine, complete, in calefaciendo ueroer re= frigerando fymmetros, fiue medus, df tring $i$, vit ins tum, ut adf tritione fua bypocyitidis fucco propins ace cedat.

\section{Schinu Anthos.Schini flos.}

Schiniflos, calcfacit mediocriter, ad/tringit minus, nec plane àtcnutatis natura alienus. Vnde ad imingen= dum medicameneft, menftrua'́; agit, ab obftruetione liberans, in fométatione unpotu $\sigma$ catapla f matis affu $=$ mptus, magis coadfitringit radix, quodueroflorem uo cant, caldilius eft.

\section{Titanos Calx.}

Calx uina, uchementer adurit, adeo ut cruffas effic clat. 


\section{DE SIM PLICIBVS.}

ciat - Exitincta mox quidem or ip acfcharam molitur, at poft diem unŭ minus, fimtiter $\mathcal{\sigma}$ carnes liquefacit. Si uero abluta fuerit atq; maxime marind aqua, digea rent is potentie efficitur.

\section{Tragacantha.}

Tragacantha, fimilem gummi facultatem obtinet,

\section{Tragium.}

Tragium, planta of in fola Cretainfula, lentifco fis milis.Calida in tertio or dine, attractorie, digere étis er tenuium partium potentie, ftipites extrahut, $\mathcal{V} d r d=$ chma pondere fumptt, calculos confringit, $\mathcal{O} \mathrm{M}$ anfes ciet.

\section{De Alio Tragio frutice.}

Eft aliud Tragium hoc amarius, quod ftolopendrio adfimulatur, $\mathcal{O}$ multis locünafcitur, ad/tringit uehe $=$ menter, adeo ut fluxionum affectibus conueniat.

Tragoriganos Hircina, Origamos.

Tragoriganus, potentia origano aßimilatur, afju $\Rightarrow$ mit $\mathcal{O}$ adftrtcionis quappiam.

\section{Triobolos.}

Triobolos, refrigerat medocriter, fed terreftrts fit cando uehemens eft, aquatilis fenfim re friger at, dimbo aduerfum phlegmonorī generationes, ev alias fluxio= nes congrunt. Porró terreftris fructus cum attenuan tium partium fit, in rembus lapedes atterit.

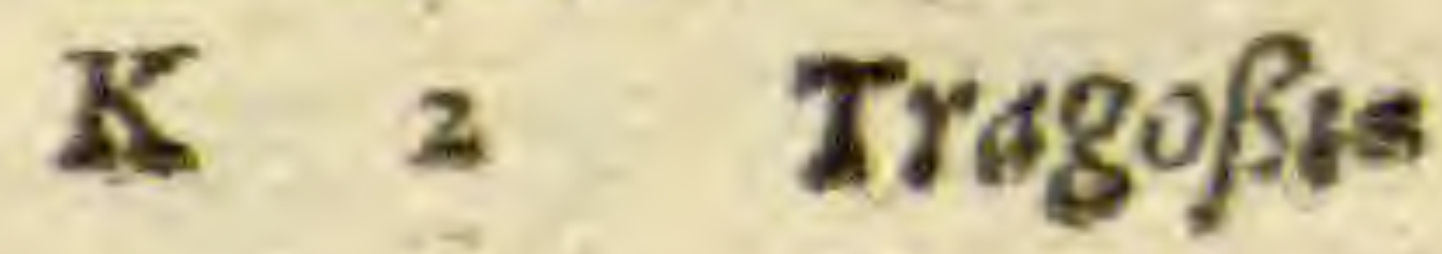




\section{PA V L. AE GIN E T A Tragoffitodes.}

Tragoßitodes, equiparatur zie, minustamen con= coquit, $v$ uentrem cmollit.

\section{Trigle pifcis, mullus.}

Trigle pifcis, fépiusconmeftus, havetes oculos $f_{d=}$ cit. Crudus concifus atqi impofitus, marinidraconis $\mathcal{\sigma}$ forpij,, dracene ictus fanat.

\section{Tripolium.}

Tripolij radix, patentia calida eft, ev acris.

\section{Triches, Pili.}

Piliuftiqualitate, ufislanis fimiles funt.

Trigon Thalaffia, $\mathrm{P}$ aftinaca marina.

Paftinace marine, aculeusimpactus denti imbe= cilli, confringit eum, $\sigma$ exciderefacit.

\section{Fex Vini.}

Fexuini, ed qua combufta necdume eft, cópofite eft effentie, impenfa deficcans atg; obftringens, quam nis

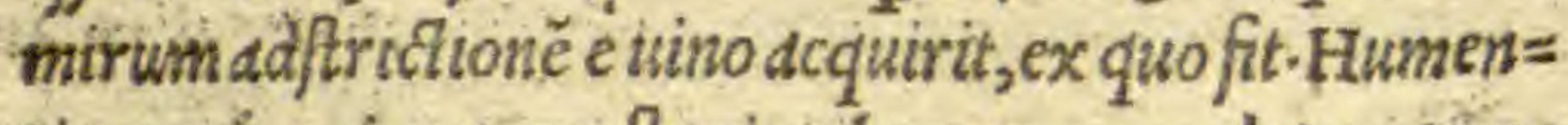
tia queq ${ }^{2}$ exiccat, o fluxionbus accommodatur, pres tered e phygetla dif cuttit, or ex uberibuslactis, fitlis

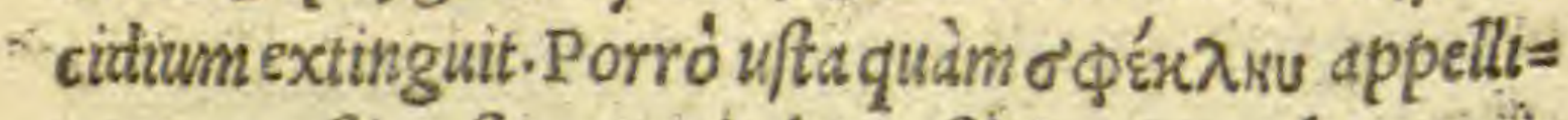
tant, catifica fit ac proinde cauflicis etiant pharmacis permifers 


\section{DE SIMPLICIBVS.}

permifcetur. Vngues leprofos exterit cumrhefind. Cü oleo lentiftino inicgram noctem per uncto co captlis cio, capillos flauos reddit. A blatain aqud, oculorumab ftergentibus potentijs mifcetur.

\section{Fex aceri.}

Aceti Fex, flocibus uini per onnia wehementio eff, magisq́gac magis adffringit :

\section{Troglodytes Paffer.}

Troglodytes $\mathrm{Pa}$ Jer, ef ommium arium minor exs ceptoregulo. Degit maximc in fepibus, quocunǵ; mo= doutare fiue affo, crudo uelcondito, duet liquato, renh̆ lapillos atterit,fimiliter o uficinis eadem preftat.

\section{DE HIIS Q VAE IN= cipiunt abliterd $\mathbf{x}$.}

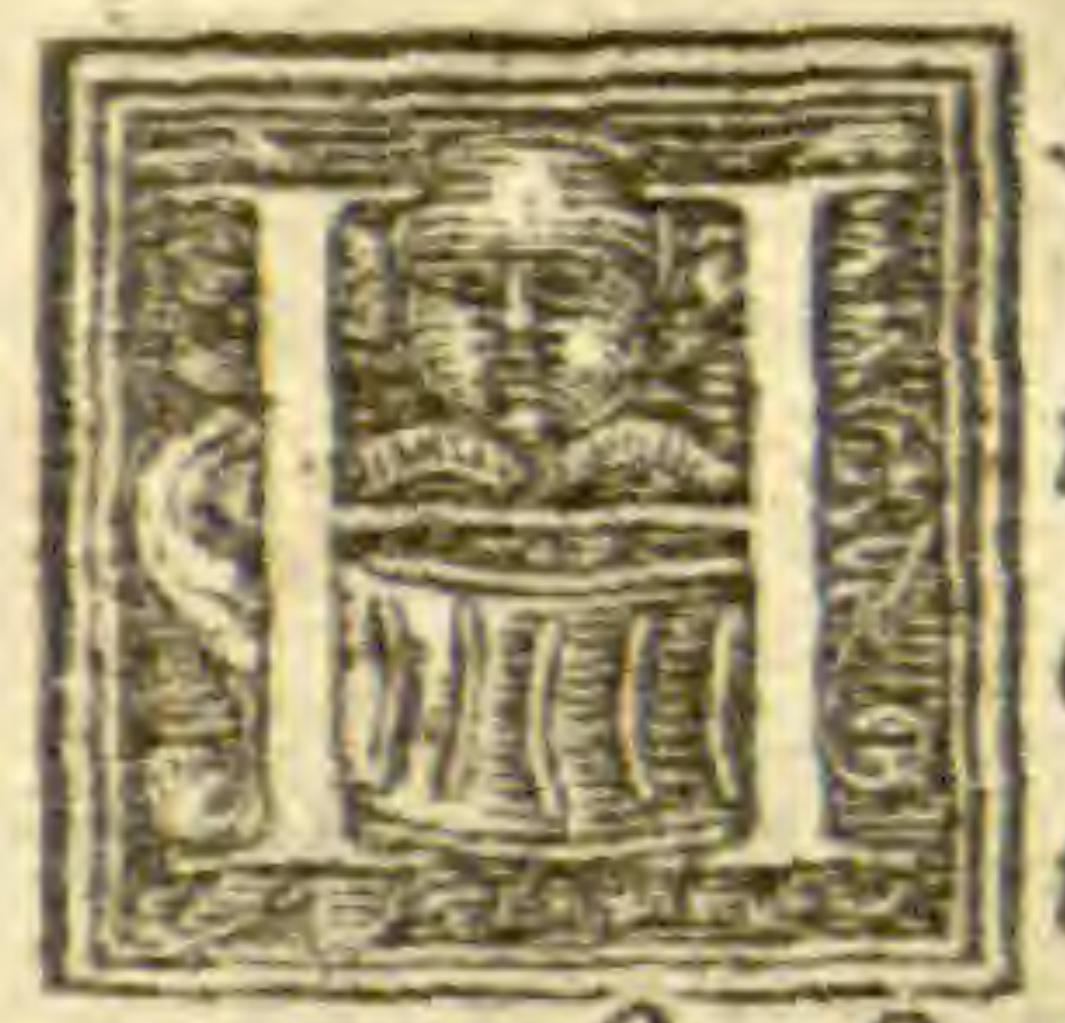

Yacinthi, radix buibo fa, ordinis eft pri mi in exiccando, in reffrigerando fecun di-Itaq; pueros, ne pubefcant diuti $\beta_{l}=$ me feruarecreditur, cumn tino ouidels = cet appofita, fructus modice abftergit o ftringit. Pro inde arquatis datur cum uino, ficcans quidem magis, minusuerorefrigerans.

Hyalos Vitrum.

K 3 Virums: 


\section{P A V L. AE G I N E T A}

\section{Hialos, Vitrum.}

Vitrum, in fubtilißimum puluerem contritü, ddeo $q_{s}$ cum uino tenut $\mathcal{O}$ albo potü, in ue fica lapides confritis gere unlgo dictur.

\section{Hydnon, Tuber Tetra.}

Hydnon, terreftram fubftantiam dominantem has bet, cumpaucula quadans partium tenuitate.

\section{Hydrargyros,Argentum Viuum.}

Hydrargyros, in re medica neceffarium non admo= dum habetur, nimirum letale, quidam adurentes, in $c i=$ neremóg redigentes, $\mathcal{O}$ alijs permifcentes fpeciebus, colicis $\mathcal{O}$ ilco confectis bibendum dederunt.

\section{Hydropiper.}

Hydropiper, catida eft herba, fed non $u \int_{g}$ ddeo ut piper, uirens or una cum femine cataplaf mate impofi ta Hypopia, $\mathcal{O}$ induratos tumores digerit.

\section{Hyoltiamus,}

Vterq; Hyoftusmus, $\mathcal{O}$ qui nigrum, $\mathcal{O}$ quiflaum femen bebet, uenenofus eft, ceterum medicine tantum utilus eft, quiflorem $\mathcal{O}$ femen album gcftat, ex tertio ordine refrigerantium.

Hypericon. 


\section{DE SIMPIICIB}

Hypericon, multorü gcnerü eft, nä eft quod Anàrd femon, $\sigma \mathrm{A}$ Atyron uocatur, de quabus in fuperioribus dictum eft, calefact uelociter $\sigma$ ficcat, propter fub $b=$ frantia partium fubtiltatem. Quare tota berbd una cum femine, twon Menfes tum Vrinas prouocat, foliare centra cataplaf fmate impof itta, uulncradd cicatricé per ducunt, arida at $q_{\text {; }}$ fuperimpofita, alia quog, bulcera $\int a$ nant, dantur o If fbiadicis bibenda.

\section{Hypecoon.}

Hypecoon, frigefacit in tertio ordinis, facultate $p_{\alpha=}$ paueriadfine.

\section{Hypoglofion.}

Hypogloffon, fuccusceft cxpreffus ex radice cifthi o Cif thari, uebementer efficax imedicamentum ado= mnes generis fluxiones, ex exiccantü, corporisg.; mem braconfirmanda.

\section{Hypophrfton.}

Hypopheftos, genus ef fullonie fpine, quemadmo

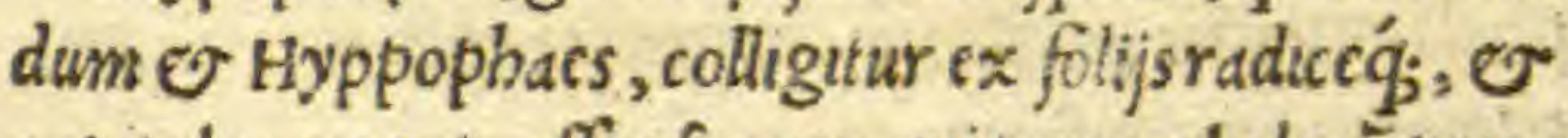
capitulo cuus expreffus fuccus, qui trum obolorüpon= dcrc cum melccrato datus, aquam e pitutam purgat . Iuuat ortbopirocas $v$ Epilcpfids, $\mathcal{O}$ que in neruis euemiunt affectiones.

\section{Hyffopum.}

Hyffopum, calefact $\mathcal{E}$ exiccat intertio orditie, tes nkiamǵg partumeff.

$$
\text { K } 4 \text { De }
$$


PAVL. AE GINETA

DE HIIS $Q V A E$ I $N=$ cipiunt ab litera $\odot$.

\section{Phaci, Lentes.}

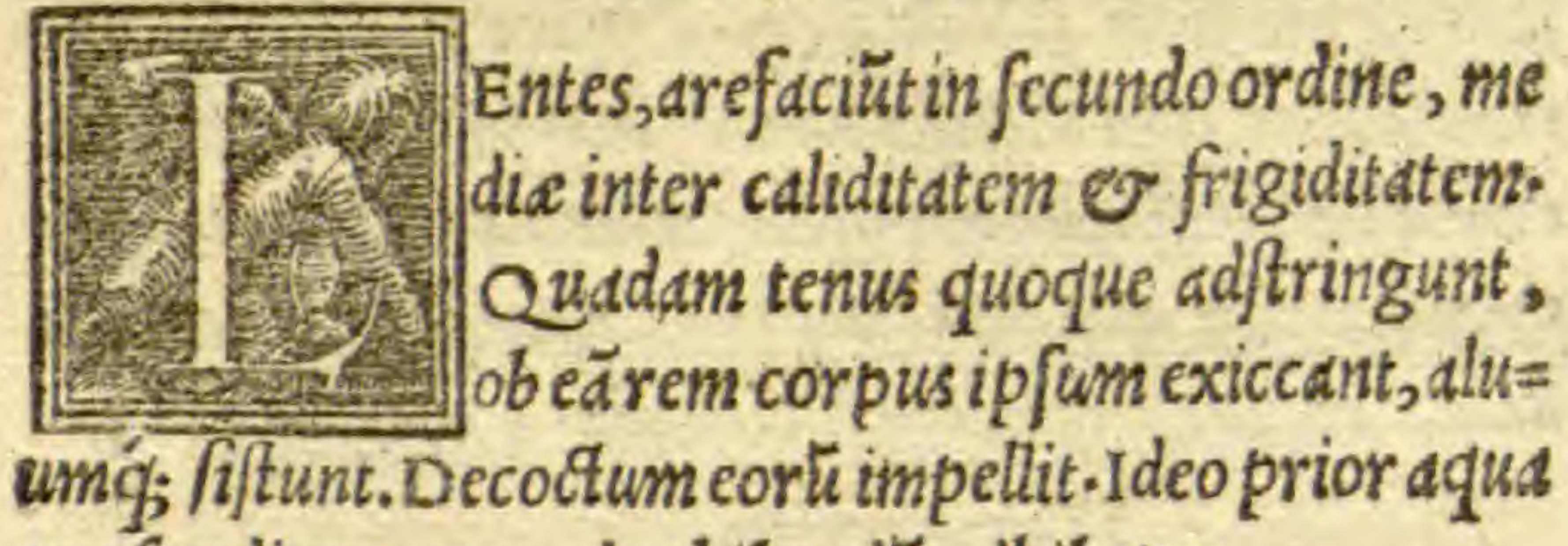
profunditur, cum ad cobibendü adhibetur.

\section{Phacos, Lenticula paluftris.}

I ens, que in paluftribus aque innatat, bumetiat o refrigerat in fecundo ordine.

\section{Phalaris.}

Phalaridis fuccus, $\odot$ folid $\sigma$ femë potd; uefice do loribus auxiliatur. Habentenim quiddam in Se partium tenuium,calidumíg.

\section{Phalangites.}

Phalangites, herba quedam fic dictd, ab eo quod à phalangij iftis alxilietur, potentia tenuium partum, proinde torminofos tuuare creditur.

\section{Phelli.}

Phelli, or maxime ex uindrijs thafis ufti inficct $f=$ mum foluuntur cinerem, qui dy]fentericis potißtmum mifcetur fucultatibus.

\section{Philyras.}

Philyre drtoris folid adfiningunt, modo quo of fyls ueftris 


\section{DE S I M L I C I B VS.}

ueftris olce. Subactd in corpore hulcerd medicdntur. Porrô eius decoctum urinam e Merfes cit.

\section{Phlomi.}

Phlomi plures funt, ex quibus eft, que $\theta$ gua $\lambda$ is aps pellata eft. Singulorum folla are facientis, o mediocri ter abftergentis funt potentic. V fus'g; sorum potißimüu adadfringendum. Quare fluxionum adfectibuscon= gruunt.Sed ov dentium quog; dolorem quidé colluunt. Phoenix.

Phoenix herbd, funt qui Rhun uocent, lolio fimilis, facultatem babetadferingendi, unde pota in uino aufte= ro, quafcunq; fluxiones facile fiftit.

\section{Phoenix arbor.}

Phoenix, tam arborem fignificat, quàm fruetum, ca lida eft mediocriter, firma'; , Oftomachoconueniens, fiue intro fumatur in corpus, fiue deforis admousatur. Quod reliquumeff exea, ddftringit ualëter, ftomachi= CIS atq. hepaticis potentijs permifcetur, omnum con= Arictıßinaeft, que fructumipfum ambit tunica.

\section{Phormion.}

Phormion, fubtilium partium eft, cxtergentis क्ष or attroctorie faculttatis. Semencum melle, albugines ocu. lorwm extenuat, or infixa extrabit.

\section{Phu.}

Phu, Nardo fimilem radicem habet, fed ob plera đ; imbecillior. vrinas tamen propellit fortius, quadm que= Lbet alis.

$$
\text { K } 5 \text { pbycos }
$$




\section{P A V L. AE G I N ET A}

\section{Phycos, Alga.}

Phycos, refrigerat $\sigma$ exiccat in fecunda diftantido Habet quiddam modice acerbum.

\section{Phyllitis.}

Phyllitis, facultatis acerbecum fit, non abs re diar= rhoeas potaiuuat.

\section{$D E$ H I $Q V A E$ I NCI= piunt abliterd $X$.}

\section{Chalbanæ, Galbanum.}

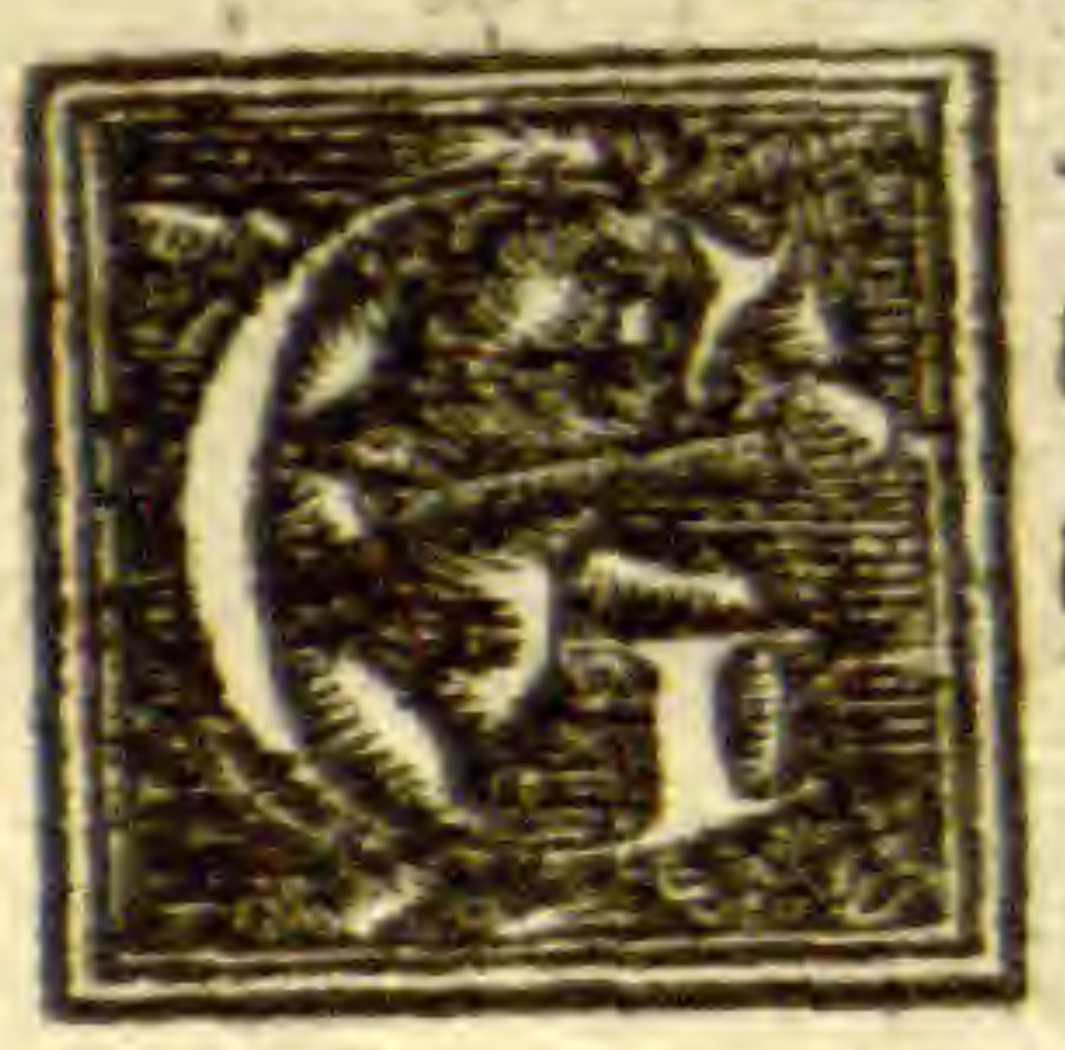

Albanum, cale facit ferè in tertia diffan $t t a, \mathcal{O}$ de ficcat quafi in fecundo, poten. tie mollendi $\sigma$ digerendi.

\section{Chalcitis.}

Chalcitis, dd/fringentis, acutecalls fice, $\mathcal{E}$ cruftas gencrantis potentie eft, partium tenu=

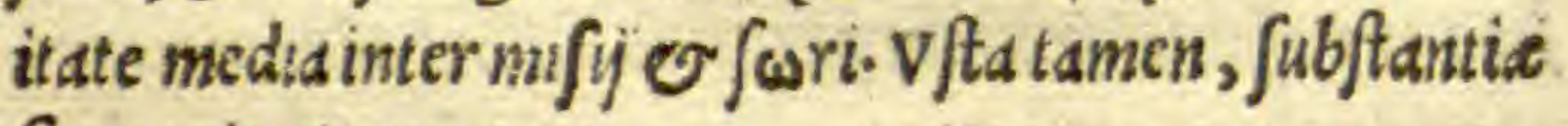
fit magis attenuants.

\section{Chalcanthos.}

Chalcanthos, aqua eft concreta in cyprimetallis, for tißimambabens ad/trictionem, cum quadam caliditate cxili. Nibil aque bumidas carnes condire at f $_{\text {ficcare }}$ udlit.Succefju temporischalcitipermutatur.

\section{Chalcos, Aes.}

Aes uftum, partimacreeft, partim dd, ringt, pro= inde utillßimmü ad cicatrices inducendas $f$ iccor $u$ corpo rübulceribus. Qu uod filltinatur, ad omria cömodior fit. 


\section{DE SIM P LICIBVS.}

chondrtlld, feris fpccies, quiddam babct fubamarü, quo fit, ut potenila fir ficciori.

\section{Chondros}

Chondros, equiparaiur tritico, preterqudam quod the habet lentoris of craßiciei.

\section{Chrifocolla.}

Chrifocollain metalls inuenitur, of preparatur in mortaitolo ex ere ribco, ex urtha pueritmpubis, trita fubinde in mortariolo urind, aut certe are caldo. Sic preparata, difcuttenté $v$ exiccan ẽ potentiă acqutrit, pius alijs metallicis. Obeamq; rem hepar in primis $a b=$ fiergit, hulcerumí; carnes fine mordicatione reprimit. Dolofis quog; o maleficis bulceribus accommoda. Quod $f$ aduratur, multotenuiorem efficies.

\section{Chrifocomx}

Chrifocome uel Chryfites, acrè fimul er adftringen tem habet ficultatem. Quare non admodum multi ufus. eft in Medicinis, preterquă in peripneumonijy, $\mathcal{O}$ affe Clibus hepaticis, quo modo in melicrato cocla datur. Commonet o Menfes.

DE HIS QVEINCIPIVNT $a b$ titerd $\mathrm{*}$.

Pfeudobunion.

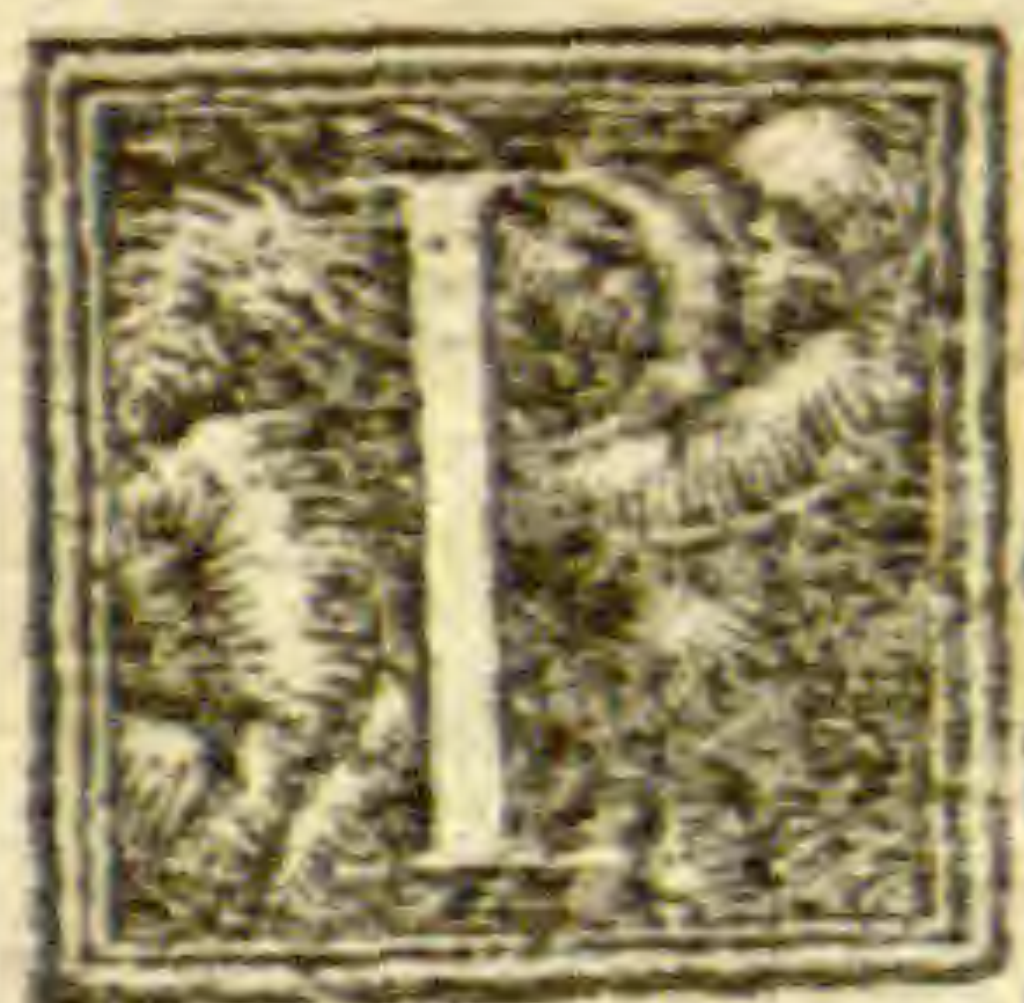

seudobunion, facultate fimile Bunio, cd efacit, disgerit, $\mathcal{O}$ abftergit - Tormina utag of frangurias, o coftarum dolo res folutit. Difcutit $\mathcal{O}$ choradas tum $\int \mathrm{s}$ Ie uino cat taplajmate illita. 


\section{PA V L. AE G I NET A}

\section{*udodictamum.}

Pfeudodictamum, imbecillem dictamiuim babet. Pfillion.

Pfillif femen, fecundieft refrigerantium ordinis, is ficcando uero e bumectando fymmetron.

\section{Pforicon.}

P foricon fic fit D uplum chalcitis mi (cetur lytargy= ro, utris qुp prius aceto folutis, inqu; ollam nouam conie= ftis, deinde diebus quadraginta fimo obruitur, ficq́, pharmactum fit preparatum exiccatorum partiumq. tenuitun, choelciti minus morddx.

\section{DE HIS QVAE INCIPIVNT ablitera $\Omega$. \\ scimon.}

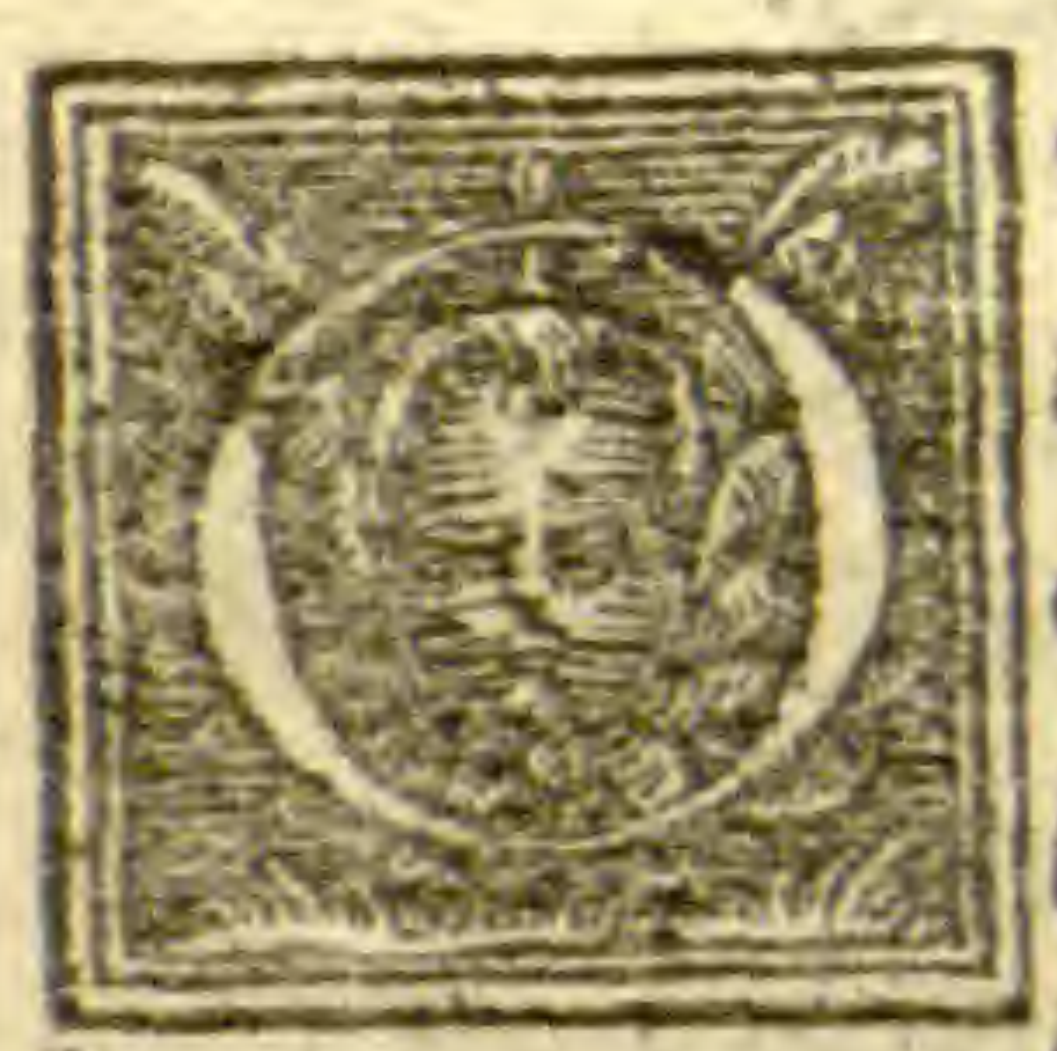

Cimon, ex fecundo ordine eft calleficien tium, habet uerorecrementiciam quan dambumiditatem. Qu fit, ut intracor pus fumptü, noxiüfit - Extracataplaf $=$ mate illitü, tum digerit, tum cöcoquit. Ocimoides.

Ocimoides, quam alij phileterion uocant, radicem babet nullius frugi.Semen attenuantium partium eft -Et fine mordicatione exiccans.

PAVLI AEGINETAE DE

$$
\begin{gathered}
\text { SIMPLICIBVS } \\
\text { EINIS. }
\end{gathered}
$$




\section{$P A V I V S A E^{7}$}

GINETA, DE RATIONE VICTVS,

\section{GV LIELMO COPO BASILI}

ENSI INTERPRETE.

\section{Deciborum facultaribus}

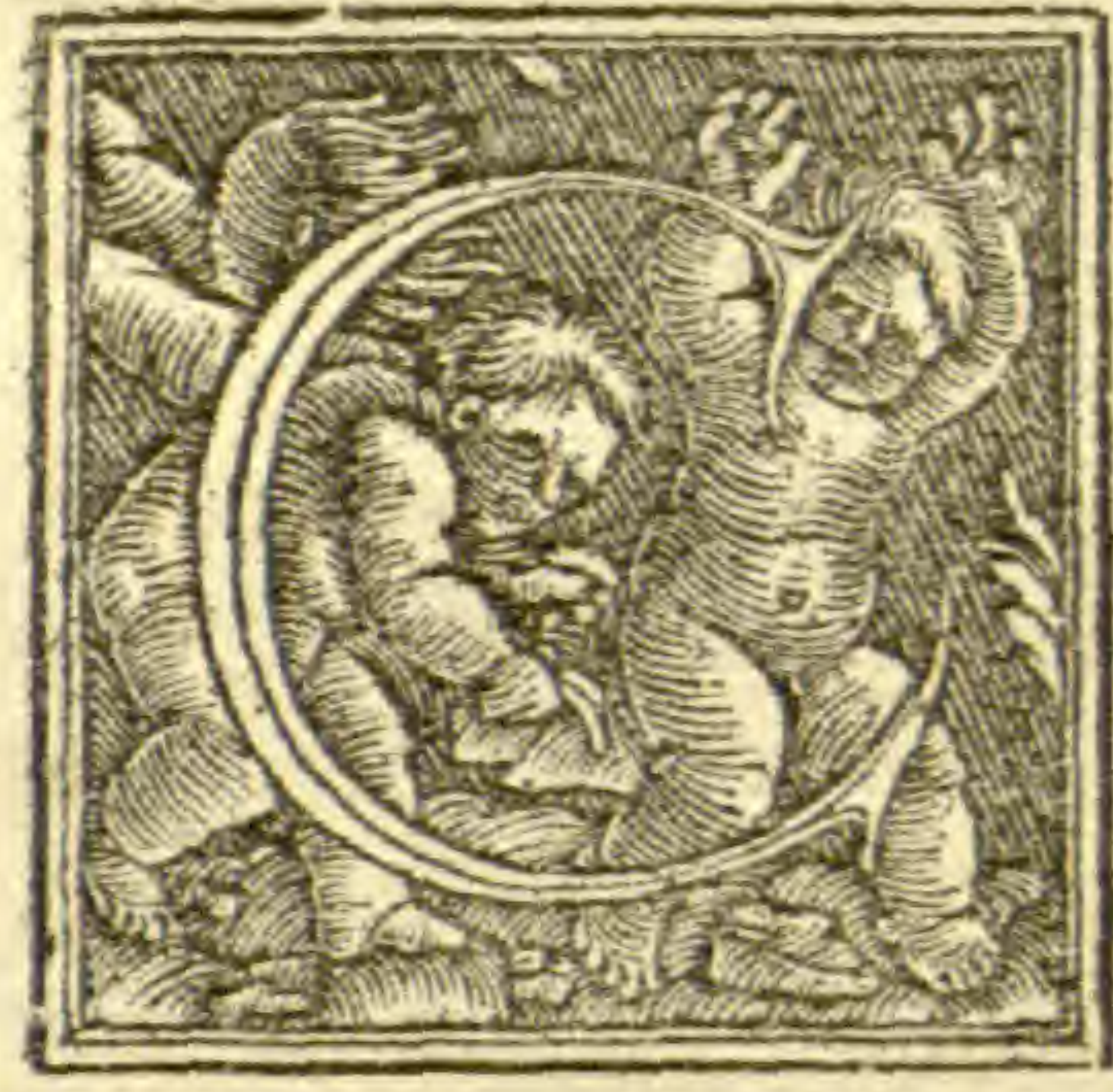

ONSE QVENS eft, ut de ciborum facultatis bus fermonem, quifallu= brtum qquog; pracepto $=$ rumpars eft, predictis fubnectamus, fi prius $\vec{q}$ particulatimip fos profe quemur, patca quedam pramiferimus, quippe oportet, $\sqrt{1}$ quicquam aliud, cióo rumno/fe facultates. Igiturcibi, quibus extenuandi uis eft, meatus aperiunt, bumores ob nimium lentorem adherentes, abftergunt, craffos incidunt $\mathrm{e}$ attenuant. Qunod fidiutius quis cis utatur, fero fas, biliofas q́; cunu labit fuperfluitates. Atq; $f$ adbuc abundantus ingeran tur, melancholicum anguinem conftitutut. Quamob=

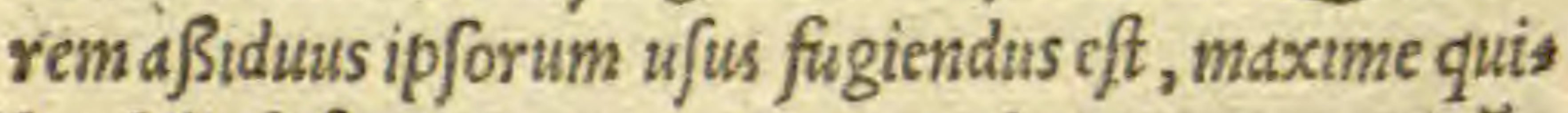
bus biliofa fueritnatura. His uero dumtaxatcongruüt, quibus pituitd, o crudus, o vifcidus, at q; crafJus bus $=$ "Hor cumulatus efl. Qui ucrocibi impinguandi uim obtinent

\section{Mo. Bot. Garden,}




\section{P A V L AE G INTA DE}

obtinent, ij firmum prebent alimentum, atq; fi tmuen= triculo, iccoreq́; probeconcoquantur, bonum angui= nemgenerant, fedlenem o iecur obftruunt - Horum ueroipforum aly craßiores humores dumtaxat ficcüt, utlens, alij uifcofos, ut malue, alij ercraffos or uifctdos ut quibus tefta protegmine eft. I gutur uictus extenuans ad bonamudesudinem tuendam, multo tutior eff $\mathrm{eo}$, qui craßstudinem induct. At quidexiguum alimentü pres $=$ bet, nibil uel firmitudinis, uel roborts, corpori prefitat.

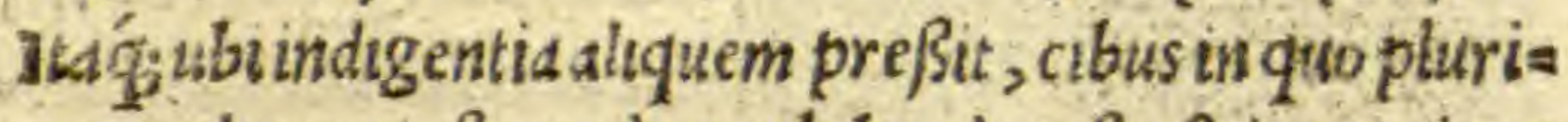
mum alomentieft, modicc exhibcrdus eft, fed precipue illis citranoxanda iur, qui excrcitationt operam dant, or qui quantum libuert, dormunt - Qzicung; ucro antecibim exercitarinon poffunt, alimenta que craffos fuccis ficiunt, fugeredebent. At neq; inociodegentes, buu fcemodicibosadmittant. Porrôut perpetuumoci= um bone ualetudini maxime aduer faiur, ita mediocris moius fummo pref idioeft.

Medie ucro matcrie dlimenta, onnius preftantifis $m:$ funt, utpote que medtocris fubftantie fanguinẽ pro ducunt, Atq; ut builuf femodi edulia corporibus noftris commodisina funt, ita uiciofum fuccum generantia, ma xime noxit, fcmper 'qf fugtenda funt.

Summopere uero ut tanda ef alimentorum udrietas, precipue ubicontrarie fuerint eis fucultatcs, nidim que fic ingeruntur, coctionrefiftunt. 


\section{DE OLERIBVS. Lactuca.}

Actuca, manifeftercfrigerat $\mathcal{O}$ bume: (2) Ctat, at $q_{\text {; }}$ idctrco fommum quoq; allicit. 5. 15 ivtueroexolcribus ip fa maxime nutri= 1. cndiuim babet, ita optimi fueci fangui= nem facit.

as Intubus.

Intubus re frigerandi, bumectandigi, uim obtinet, at Iaciuca inferiorem.

\section{$\checkmark$ Malua.}

Malua refrigerat quedem, fed obfcure, magis uero bumectat, at $q_{;}$ob uifcofital cm aluum ducit.

\section{Beta.}

Beta, abftergendo aluum mouet, unde ficrebro edd tur, ftomachum uellicat, di $q_{;}$iecur $\sigma$ licnem ob/truit.

Braffica.

Braßsica, bis quidem cotta, commefta q́, uentrem co hibct. siuero femel, neq; ddmodum coquatur, at $q_{;}$ex oleo, Ð garo, aut fale edatur, ducit pottus aluü, quem= admodum tus eius purgandi poteftate lentium cremore preffantius eft. At manifeftus magisq ; ucntrem mollit, marinaBraßica, nam of $f a l \sqrt{a}$, o fubamarulenta est, quapropter auroni quoq; fubducendl aluum poteftas eft, é quòd plurima falfedine participat. Braßicauero ftomacho magis conuenit, utpote que mediocrē obtinct adfringendi uim, sc lac quog; $\odot$ genitale femé pducit; Blitum 


\section{PAVL: AEG INTA DE}

$8 \%$

\section{Blitum.}

Blitum or dtriplex, humore quidem abundant, $f c d$ - exigutum dimentum prebent.

Omnia dculeatd, ut cardus, of fufus agreftis, al $\phi_{s}$ alia buiufcemodi, ftomacho idonca funt, exceptacina =

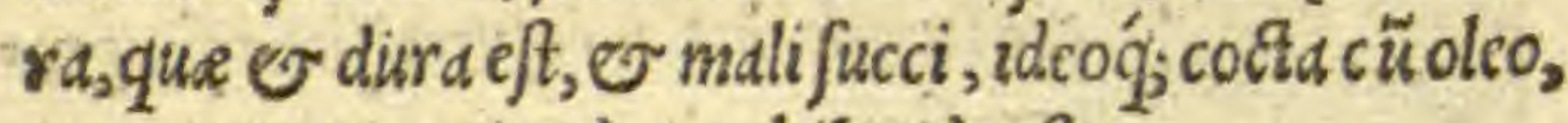
o garo, 0 coriandro, exhiberida eft.

\section{$\checkmark$ Appium.}

Appium, bippofelinon, Sion, Smirnium, urine cien de facultatem babent, fed Smirnum or magis odord= tum, evacre est, $\mathcal{O}$ urinam or men/frua mouet - Ap= pla uero fuduiord funt, ideoớf fromachoaptiora.

\section{ar Eruca.}

Erucd calide cft, $\sigma$ genitale femen producit, unde

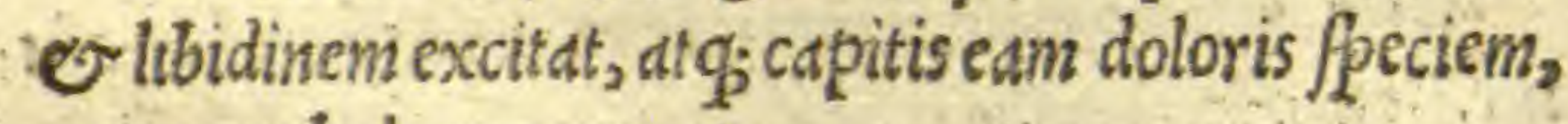
quamcephaleam nocant.

\section{Nafturcium.}

Nafurcium, ocymium, finapi, calida funt of acrid, op precipue nafurcium, funt werococtudefficilia, ftos macho inepta, or mall fucci.

\section{Vrtica.}

Vrtica, tcnuibusconftatpartibus, aluum ducit, atd exiguum alimentum prabet.

\section{Gingidion.}

Gingidion, fimile fcandici eft, haud parum stringen tis, amarulenter q qualitatis babens, fromacho aptum, ut faftidiofis quoog aptü fiex accto mäjum, fedtcorpis 


\section{RATIONE VICVS.}

parumalit. Que uero fylueftridalera kocantur, aduis, cioforum humorum generationem nibul reliqui factüt.

\section{$\sim$ Capparis.}

Capparis, o appetentiam cxcitat, o iecorislies nis $\tilde{j}_{\text {; }}$ obftructiones folutit, ac pitutuam educit, fumenda wero eft in prima menfa wel ex oxymelle, uel exolcoer aceto:

\section{$D E C X M A R V M$ natura. Blicorum.}

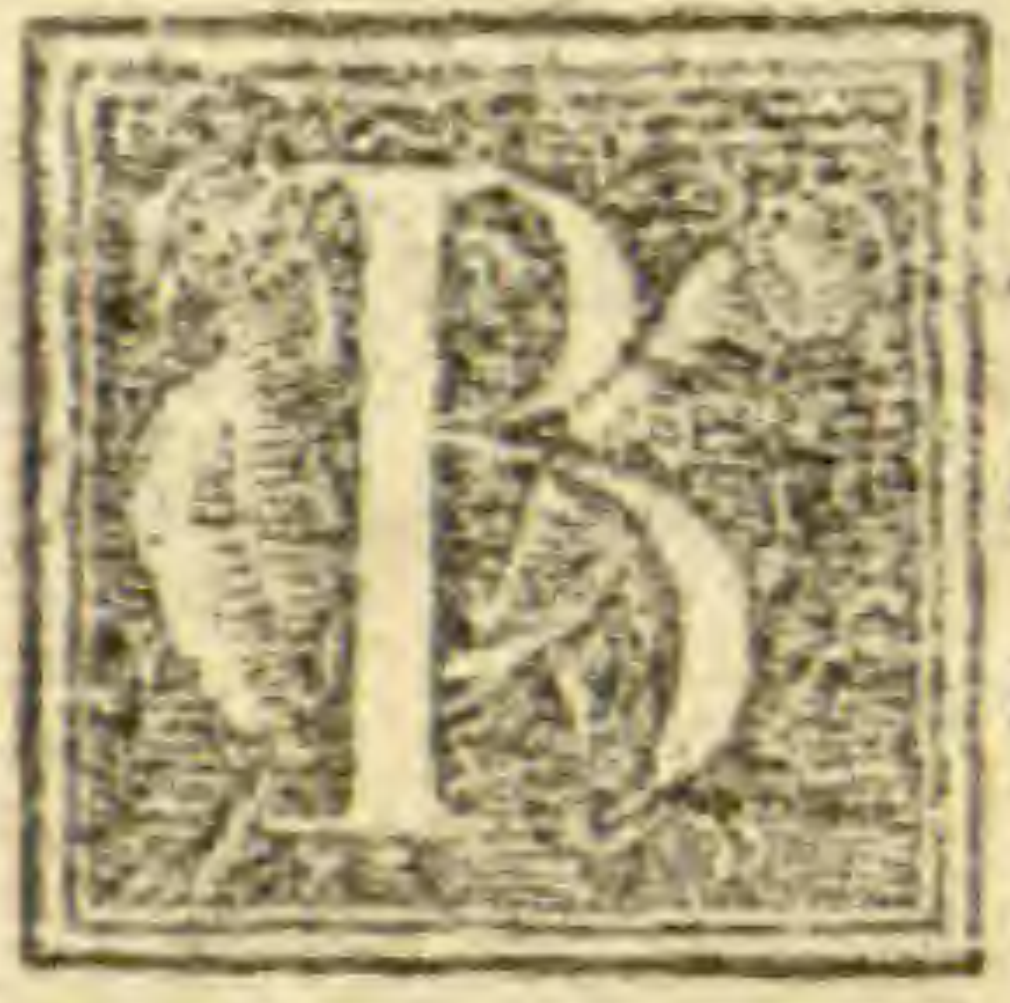
Licorum, laclucarum, driplicis, mals $u r$, bcte, plantaquidè bumsda c $f t . c y=$ ma ueroarida, rapa, finapi, radiculd, nafturcium, pyrctrum, Braßted altágs calida, plantam quidé aridam habeãt, cymam uero hus midam. Fructicofarumuero plantarü Cyme, $\mathcal{F}$ que in paludibus or que in ortis nafcuntur. Ité qua à brio= nia decerpuntur, ftomacho quidem apefe funt, o uri ndm monẽt, exiguum uero dimentum prabent, quod/s probeconcoquantur, magis quam que ab olcribus fis $=$ muntur alunt, Similiter de Chamedaphies Cyma fenti endum eft.

$$
\begin{gathered}
\therefore \text { D E R A D IC I B V } S \\
\text { quecibirationemobtinent. }
\end{gathered}
$$

\section{Bunias.}




\section{PA V L. AEGINETA DE}

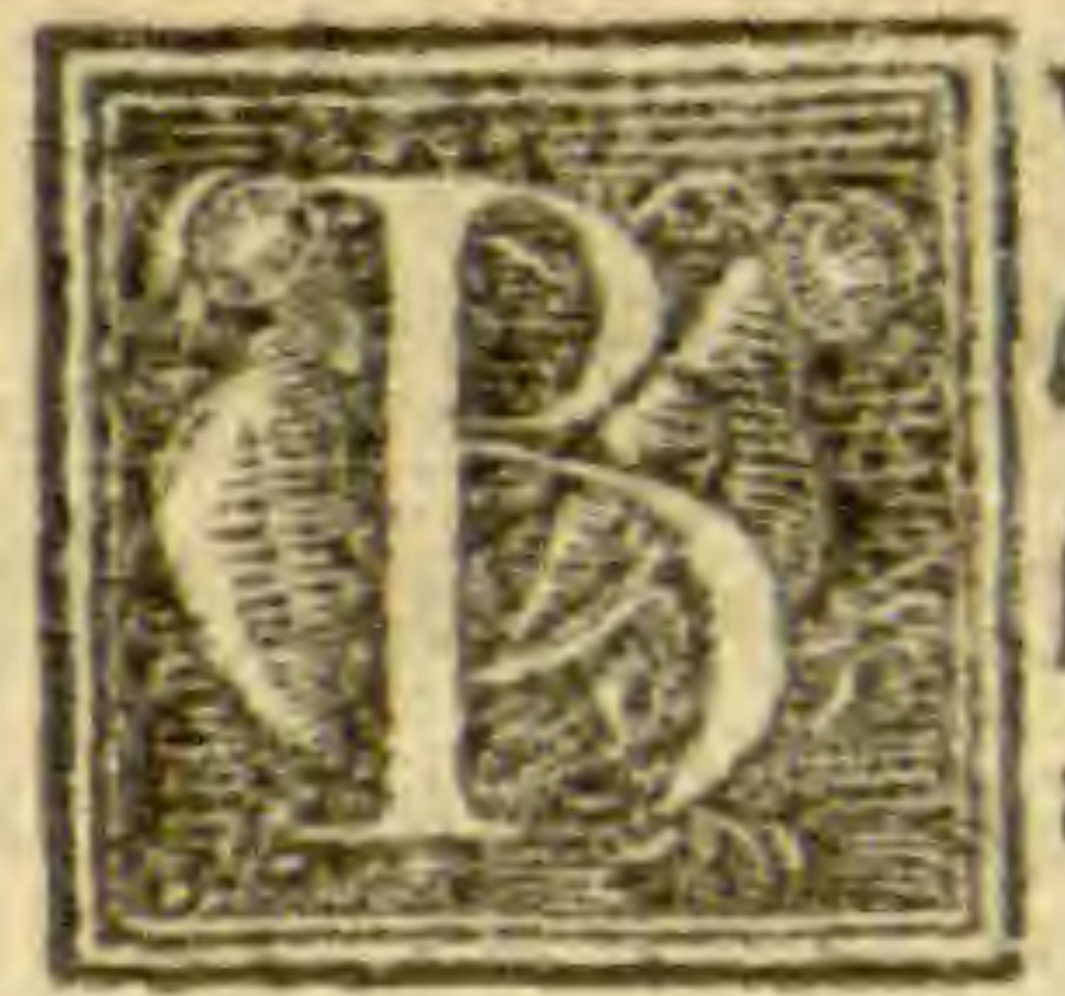

Vnias quidem o Rapa, fi bis coquan= tur, hatud minus reliquis oleribusalut, fedfitugitcraffumdintur, craffumbu= morem conciliant.

\section{Bulbi,}

Bulbt aftringunt, abftergunt, appctentiamexcitant, ftomacho robur addunt, $\mathcal{O}$ uI $\int \mathrm{f}$ fos ex Thorace bumo rescducunt, quod/i bis coquantur, plus quidé alimen= ti preftant, fcd nubil educunt $\cdot \mathrm{N}$ am quod amarum in $i=$ p fiseft, ita abijcitur, preterea ficrebroquis eis uta= tur, genitale femenaugen, $\mathcal{O}$ Venerent impendio ex = citant, flatus quoq; faciunt $\mathcal{O}$ tormisa. At ex garo $\mathcal{O}$ oleo fumpti, fuauj im $\int u n t$, o bon aliment , atque cocti faciles.

\section{Paftinacx.}

Pdftinsca, dauci, ff $f$ eris, radices, rapis minus alunt, funt uerocalide odorate $q_{;}$, atq; urtnam mouent. Abus: tentibus uero $\mathcal{O}$ coctu difficiles funt, $\mathcal{O}$ utciatos $h u=$ mores pariunt, procommuniradicum ratione.

\section{Agreftis paftinaca.}

A grcftis paftunaca, d nonnullis $\mathrm{D}$ aucus appellatur, or maioremcitande urine uim habet.

\section{Radicula.}

Radicula attenuatorte, calfactorié f. facultatis eft, fed ante allos cibos exolco $\sigma$ garo ducęide aluigra= tta, funcinds eft, poft cibum autem ntequaquam,

Cepa, 
RATIONE VICTVS. 74

\section{Cepa,Allium,Porrus.}

Cepa, Allium, Porrus, ampclopraffon, eo quod dcri

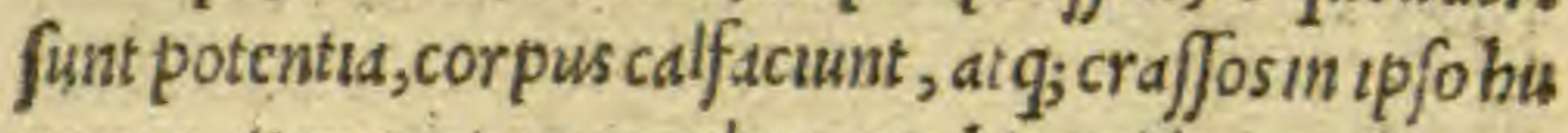
mores attenuant $\mathcal{\sigma}$ incidunt, $\mathcal{V}$ bis quidem coeta pai lum, incocta uero nihil alimenti preftant. At uero alliü optlationes folutt, or difcutëedımagis uim obtınet - Am pelopraffa autem, utpote agreftia ficciora funt.

Porrò in uniuer fumómma cruda olera, contis peio ris fucci funt, magis $q_{;}$fuperfluo humore abundants $\mathrm{Q}$ ue uero uel miuria, ucloxyhalme condita reperums tur, ftomacho aptior a funt, er appetentiam magis mo uent, accrudos humoresincadunt, fed coctudifficilia funt, $\odot$ fi quis eis abutttur, malos fuccos producunt.

$$
\begin{gathered}
\text { D E } T \text { V B E R I B V } s \text { : } \\
\text { Tuberumfuccus. }
\end{gathered}
$$

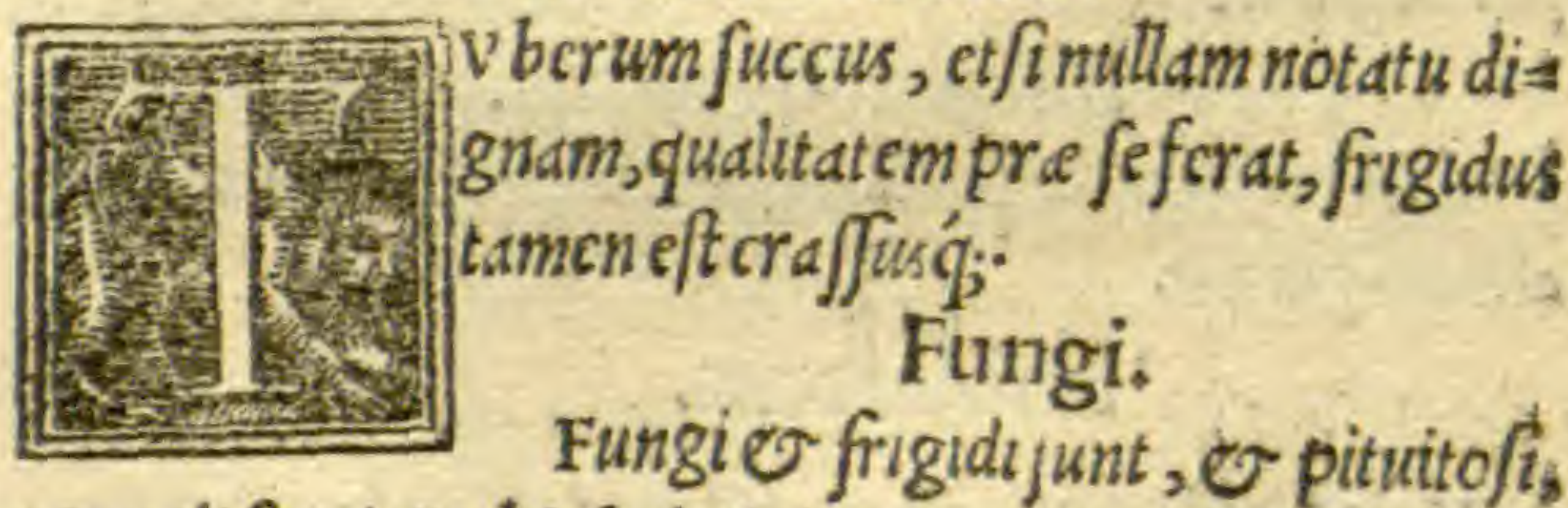
of malifucci. Ex his boleti, innocentiores funt, or ma = rifefte qualitstis expertes, firccte coquantur. Deinde ammanite ab ijs fecundũ locum obtinent. A balijs ucro fung is abftinere oportet, quippe cum multi exip/is les tifert fint, ac ipfi quog; boleti, nifipercocluedätur, pte fens periculum feperumero minantur.

DE FRVMENTACEIS.

I. 3 Excis 


\section{PA $\nabla L . A E G I N E T$ DE}

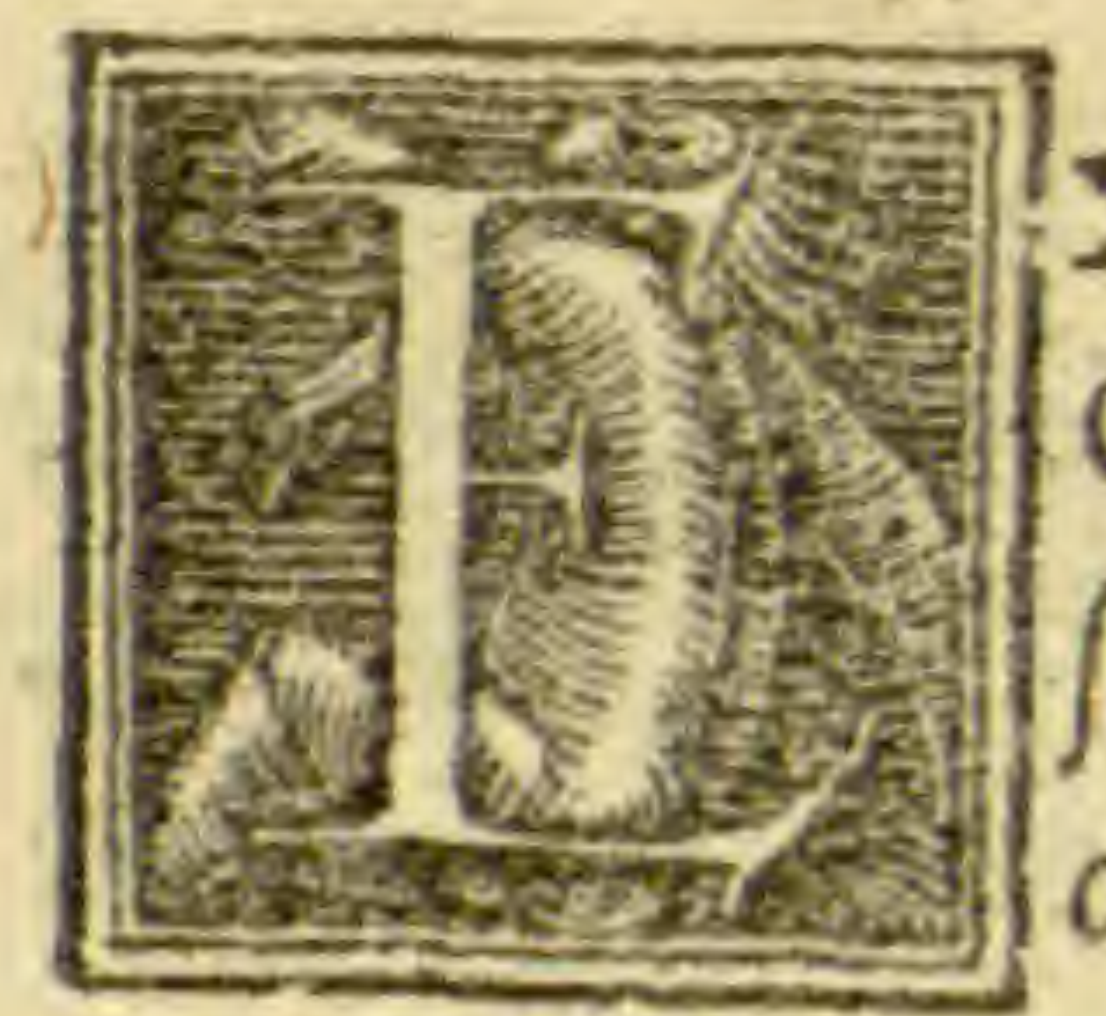

$\mathrm{X}$ eis que frumentaces appotlanitur, Chondrusmenfis conuentt, o vifco= fumbumorem parit:Atquiablutusui= coofuseft, eo quod facilecraffus euadit, at $\tau_{\text {; }}$ incoitus permanet . Melior iero eff fuccus ipfius, fitotus uti ptifana probe coquatur.

\section{Alica.}

Alicd, in reliquis quidëchondro baud abfimiliseft, fedmagis alum fupprimit.

\section{Triticum.}

Triticum, clixum quidem coefludifficle eft, $\odot$ ina - Att, ubiuteroconcoctum eft, udent 3 Simam prebet alt $=$ montam. At in panemredactum, inflandiuim, $\odot \mathrm{co}=$. ctionis difficultatem dę̧ont, utpote fermento, falléf; participans.

\section{Siligineus panis.}

siligineus panis, quem filigutem Greciuocant, omni, allomagis alit.

\section{Similaceus.}

Mox Similaceus, fic enim femidaliten interpretor, dcinde cibarius, Greci fyncomifon dicunt. Sordidus dutem, id ef, fur fureus, minus quidem allt, fed magis $p$. uentrem defcendit. Amy'us Qdnis exiguum alimentim prebet, quemadmodume lotus.

\section{Hordea.}

Hordea reffigerandi, abfergendiǵ; wim obtinent, 


\section{RATIONE VICTVS.}

cum ueroad modum ptifante. Coquntur, humectant, fricla uero ut in polentts fit, deficcant. Polenta per ef/a tem ante pallueum exaqua potuiddta, fit medetur.

\section{Hordeaceus panis:}

Hordeaceus paris, imbecillior eff, or exiguiali = n:cnti.

\section{Maza.}

Mazd, coctudiffucliseft, or magishordeaceis $p d=$ nibus influt, cur $f i$ mel quoq; acceffertt dluum moust. Bromus.

Bromus, calidus $e f t, \mathbb{e}$ cxigut alimenti. Militum.

Militum, or panicum, frigida funt $\odot$ ficcd dt $q_{;} p a=$ rum alunt, 0 uentrem cohibent. In totum uero, miliü praft:niuus eft panico.

\section{Oriza.}

Orizd, coltu difficilis eft, parum alt $\odot$ uentrem $a=$ fringit.

\section{ptifana.}

Ptifana, fic coquitur, uni eius parti, aque quinde= cim, at ; paulam oles adijce, ac poc/t bullinationemace tum quoq. Cum ueroexacte tam cocia fuerit, falem te $=$ nuem.Sunt qut porrum quog; al $q_{;}$anetum addant $\cdot \mathrm{Ce}$ terum Bromus o Chonarus, baud aluer quam ptifana preparantur.

L 3 De 
P A V L AE GINETADE

$$
\begin{gathered}
\text { DE LEGVMINIBVS. } \\
\text { Lens. }
\end{gathered}
$$

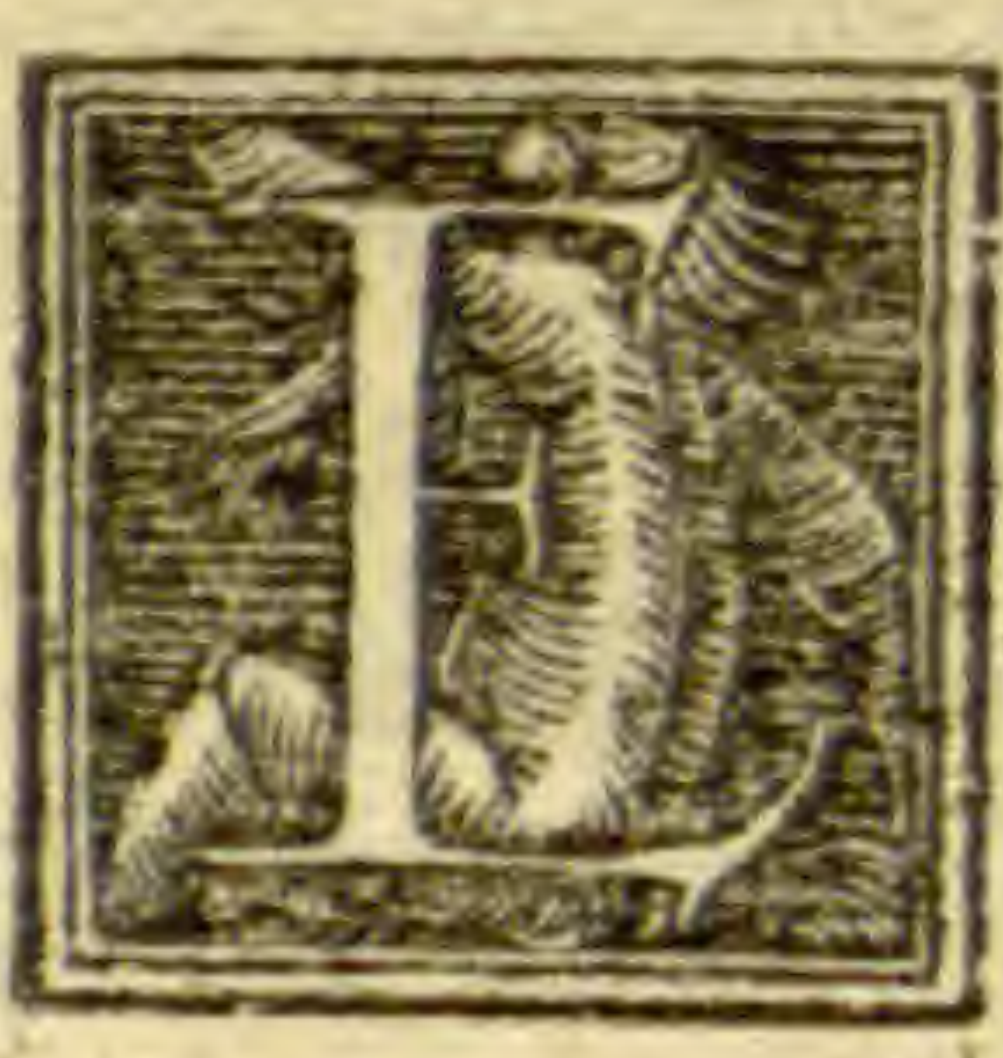

$\mathrm{X}$ leguminibus lens quidem mali fucci eft, o atrambilem part, uentrem ds ftringit, maxime bis cocla - Decoctum ueroip fius cum oleo $\mathcal{\sigma}$ garo potuida rit, uel fatureia, uel pulcgium adijciendume $e f t$.

Fabx.

Fabe lenes funt, $\mathcal{O}$ inflant, dc dbftergunt, dc Aegy ptia faba, $\sigma$ calidior eft noftra, $\sigma$ fuperfluitates plu= res relinquit.

\section{Pifum.}

"Pifum, molle quidem eft, at $q_{\text {; non }}$ adeo inflat.

Cicera.

Cicerdinflant, abftergunt, genitale femen augent, venerem fimulant, calculum ter üt. Ac fricia flatü qui dem amutunt, fed difficulter concoquuntur.

\section{Lupina.}

Lupira, funt coctudifficilia, haud facile tran fmits tunttur, o fuccum crudum partunt.

\section{Fonum Græcum.}

Fonum Grecum, antc clbum affumptum, $\mathcal{O}$ cales facit, $\sigma^{2}$ aluum foluit.

\section{$\checkmark$ Cicerx Fafeli.}

Cicer e fafeli $q_{;} ;$i maccrentur donec radices proue yeniant, atque cxgaroante cibum fumanitur, uentrem fubducunt, 


\section{RATIONE VICTVS. 76}

fubducunt; atgf magis qudm foenum Grecun alant.

\section{Fafeli,Siliqùx.}

Fdfeli tamen, que e fllique appellantion, fintegri cum filqquis, uiridesq́g, ingerantur, plures [uperfuutus tescumulant.

\section{DE TEMPORARIIS fructibus. \\ Cucurbita.}

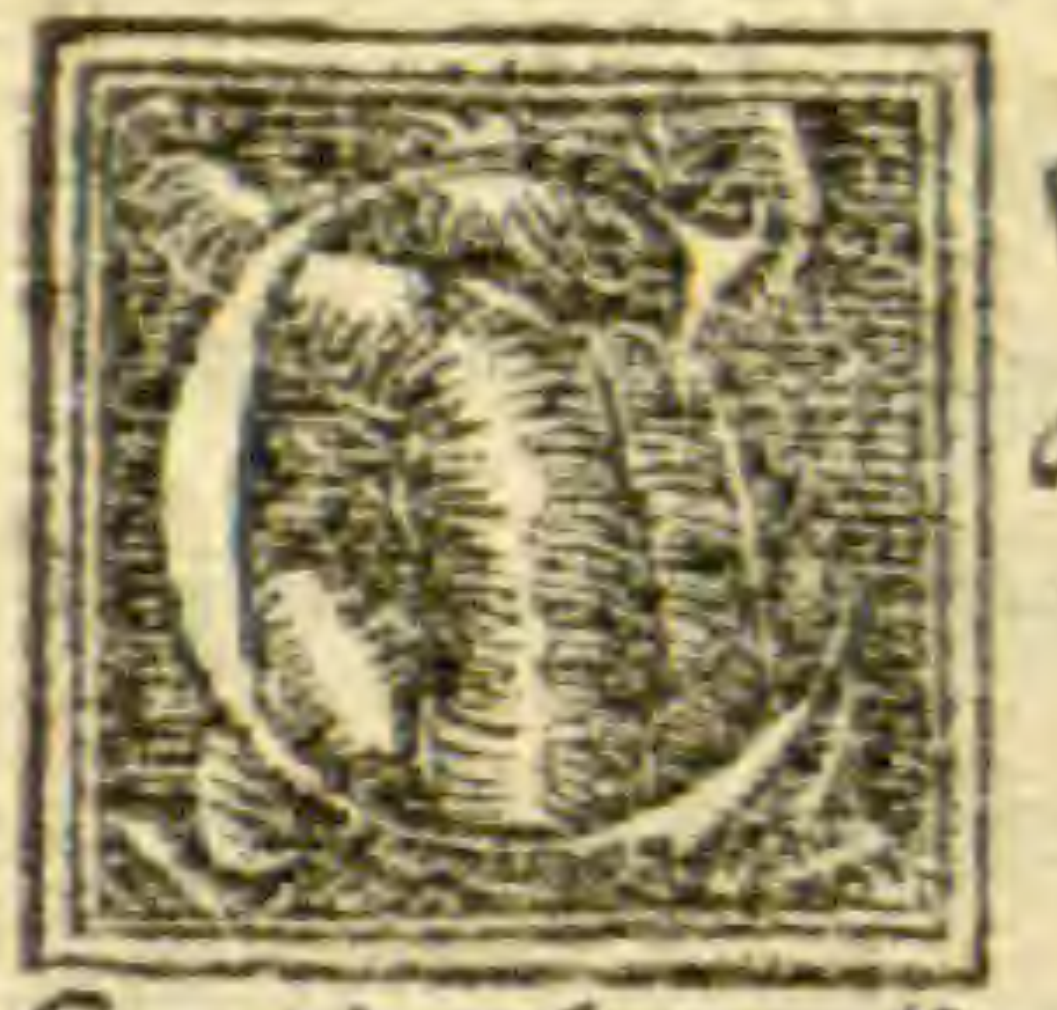

vcurbita refrigerat, humectatóf. adóf; a!uน̆ mouet, fed paulü alimétiprebct. $\sim$ Pepon.

Pepon, int totum $\mathcal{O}$ mall fuccicft, $\mathrm{O}$ frigiaus humidus'; , ac uomitioncm mouet, quod fi non concoquasur, choleram tnducit, cius femen urinamcis et, calculos renum conterit, $\mathcal{E}$ in totum uchententer abftcrgit.

\section{Melopepon.}

Mclopepon, omnia que de pepone ditta funt, inibe= cilliora obtinet.

\section{Cacumis.}

Cucumis, minus qudem pepone refrigerat, bumes ctat ${ }_{j}$, sed uebementius urinam ciet, o utx contoquix tur, quod etfic confequtur, malitamen fuccieft.

Atq;in funme, onnes autsunnales fructus, refrige $=$ L 4 rant 


\section{PAVL. AEGINETA DE}

rant, O humeclant, of funt cxigti alimenti, e mali fuccio

\section{DE A R B O R V M}

\section{fructubus:}

\section{Ficus, Vux.}

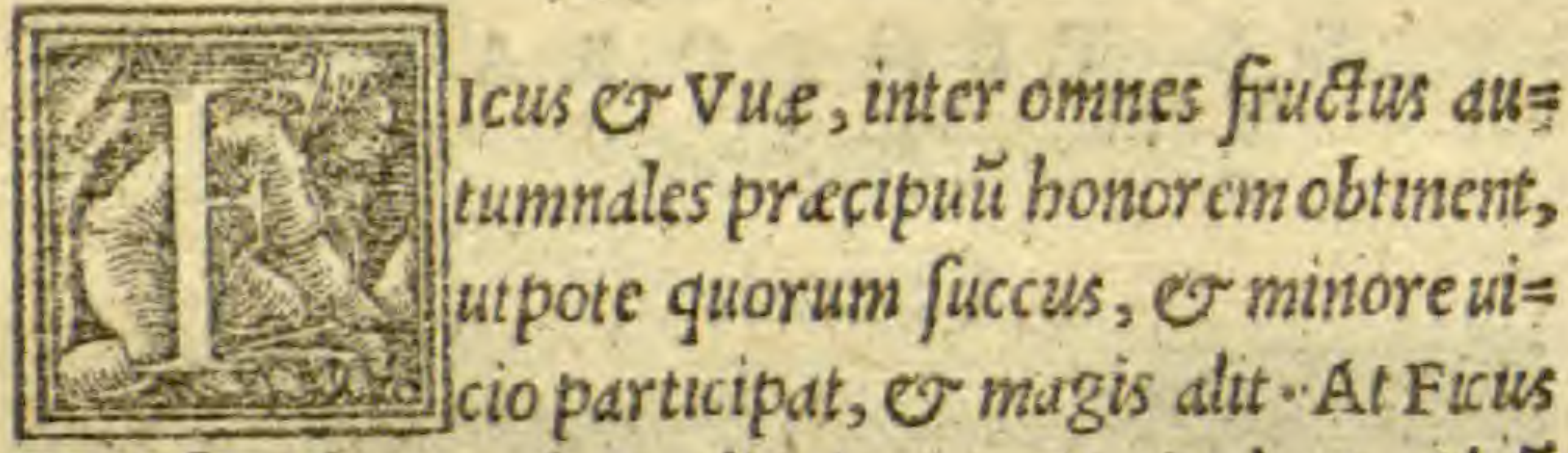
Vuis funt fucct multo melioris, atq, magis alunt . Aluü mouent, urinamcient, renes purgant, maxtme qua fue rint admodim mat ure.

\section{Carice.}

Similiter or Carice, flatum tamen or improbum fanguinem faciunt, atg; iccirco, fincrcbrouf if fuerviut, pediculos pariant.

\section{Vux.}

Vue, retent\& qudecm non colscoqunutur, o malü fuccum reddunt, pcraluum uero dccedte, moderation rescuadunt.

\section{Vuæpaffix.}

Vus paffe calidiores, funt, flomachoóf; aptiores alds; reltquis uuis mas gis alunt, fed minus aluwin foluunt. 


\section{RATIONE VICTVS.}

Mora uchementer bumectant, mediocriter'́; refris gerant, atg; in prima menfa ajJumpia, uentrem molli= unt, baudquaquam ftomacho aliena, fed parü alunt.

\section{Cerafea.}

Cera fijs, dulciora quidem alsum citant, at fomacho noxia funt. Acerba ucro ut ftomachum magis folantur, it a minus molliunt aluum. At $q_{\text {; }}$ esdem ratio tum inuuis or moris, tuminalijs multis ob feruatur:

In totum uero, aftringericia fiue cdantur, fiue biban tur, ante alium cibum, aluum cohibent. Quibusuero uenter ob imbecillitatcm nibl deijat, ij altquid quod molliendi facultatem habeat, uelutialtera, aut pifces, aut aliud huiufcemodi premttere debent. Deinde aftrons gentia fumere, que aluum roborantia, dcor [um impel a lant, at $t_{\text {; }}$ ad fecretionem cxcitent.

\section{Nux pini.}

Nyxpini,bori, craßiq́; fuccieft, atq; efuiapta, fed non factic concoguitur.

\section{Perfica.}

Perfica mall fucci funt, ace fcunt $q$; $\mathcal{O}$ fucile corrum puntur, ideoǵ; in primis funt exhibendo, ut $\mathcal{O}$ protinus dicijcantur, ncqppropter moram in uentriculo corrum paritur.

\section{Pracocia \& Rhodacina.}

Precocia uero, $\mathcal{O}$ rhodacina, $\mathcal{O}$ armenia, per ficis L 5 meliora 


\section{P A V L. AE G I N E T A DE}

cis meliord funt, ncq; enim ace cunt, neq; fimiliter cors = rumpuntur, ac funt etiamnum delectabtliord.

\section{Poma.}

Pomorum, fuauia quidem reliquis funt caliäiord, o facile tranfmittuntur, precipue fi ucl affentur, wel elixentur. Acids autem, ut frigidiora funt, ita bumos res in uentriculo magis incidunt. Aut auftera, ftomacho quidem robor afferunt, or uentrem cobibent, fed mit = xime Cydonid.

\section{Pira maiora.}

Eiufdem poteftatss funt pira maiora at $q_{p}$ maturd, fed plus alimentı prebent.

\section{Mala Punica.}

Mala Punicarefrigerant, alunt $q_{\text {; }}$ parum.

$$
\text { Mefpila Sorba. }
$$

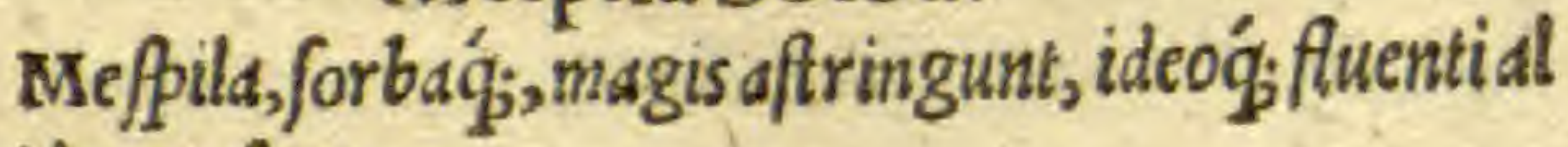
uo idonea funt.

\section{Palmx,}

Palme, nifi admodum pingues fucrint, ftomacho quidem conueniunt, fed aluum fupprimunt, atg; crafe fos uifcofos $q_{;}$, humores pariunt, $\sigma$ capitis dolorem in= ducunt.

Ex oluss druppe quidem, fomacho aliene funt, $\odot$ cra ßif fucci Colymbades nero, o que in muria feruate funt, in primamen fa affumpte, appetentiam augent, aluum citant, at $\Phi_{p}$ id magis, fi uel aceto, weloximelle condiantur. 
RATIONE VICTVS, 78 Nuxiuglans.

Nuxiuglans, auellanis minus alimenti prabet, fed ftomachomagis conuenit.

\section{Nuces recentiores.}

Nuces recentiores humide funt, at q; aluum mouét, Aride ucro, fi in aqua macerate ab interno putamine minidentur, haud minorem uiridibus babét efficaciam. Amygdalx.

Amygdale, incidendi, attenuandiớ; uim habent, un= de $\odot$ uifcerd, o thoracem purgant, maxime uero amare.

\section{Piftacia.}

Piftacia, haud dif Simuli funt poteftate, fed in leuanda iecinoris obftructione, funt preftantiord.

\section{Damafcena.}

Damafcena, ante cibum fumpta, fiue cruda, fuecx mulfo cocta, aluum foluunt.

\section{Syriaca.}

Syriacd, haud facile concoquuntur, fomacho aliend funt, 0 lcuis alimentt.

\section{Syliqux.}

Sylique, concoctioni renituntur, wentrě df tring unt, v funt mall fucci.

\section{Sycomora.}

Sycomord, manifefte refrigerant, humectantó.

Citteimali, 


\section{P A V L. AE GINE T A D E}

Citreimali externa pars acris eft, que uero ueluti carnis pectem pre fe fert, licet efuifinidoned, ulx ta= menconcoquitur . Id uero quod intus continetur, fiue acidum, fiue aqueum fuerit, uebcmenter infrigidat,

\section{Glandes.}

Glandes, frumentaceis haud minus alunt, fed conco= Culd difficles, $\sigma$ craßif fucce funt, at $q$; tarde tranfmit= tuntur.

\section{Caftanex.}

Caffanee, omni ex parte glandibus funt meliores.

\section{DE ANIMALIBVS, ET PRIMO De volatilibus.}

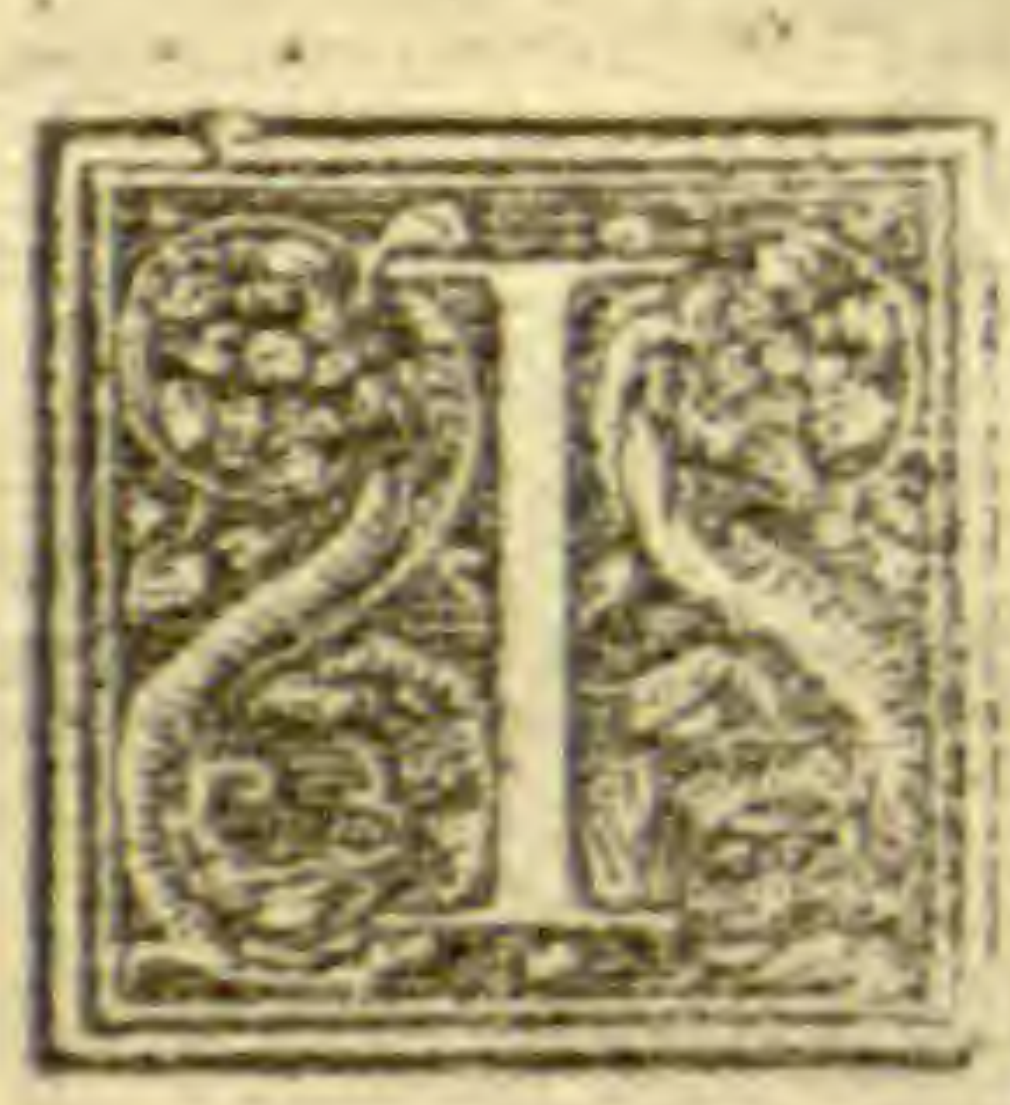

Mbecilus allmentü prabent dues, quă quadrupedes, prafortim quiam fues, $\int \mathrm{cd}$ concoefum facilius. Maxime Perdix, Attagina, Columbinus, pullus, galine, Phafiane. At exturdis, merills,par= uis pafferibus, in quo gencre pyrgite quo q; funt, foldde us alimentum eft. Id $q_{\text {; }}$ magis cxturtur $\alpha_{2}$ palumbo, and $=$ te, fed multo durius, $\mathcal{\sigma}$ coetudifficilius, at $q$; fibr ferè refertum, quodex patone accipitur. Anferis ucro or ftrutocameli caro, ommibus quas diximus, plures relun quunt Superfluitates, difficillus concoquttur, dcmptis alis, que fucci bonitate ceteris aubus batt infrriord funt. Grues, fibris plenum durumíg a'imenüu prabent. 


\section{RATIONE VICTVS.}

Intotum uero, iuniores aues weteribus funt bumidio res, cođtu faciliores, altment ualentioris, atq; per aluum factlus deijctuntur. Simili deffrimine elixe $a b a \beta i s$, $\sigma$ in fartagine frictis diftinguuntur. Item que in aridss locis, montibusớ; degunt, paluffribus, $\odot$ factlius concoquuntur, o fuperflutatibus minus redundant.

\section{DE OVIS.}

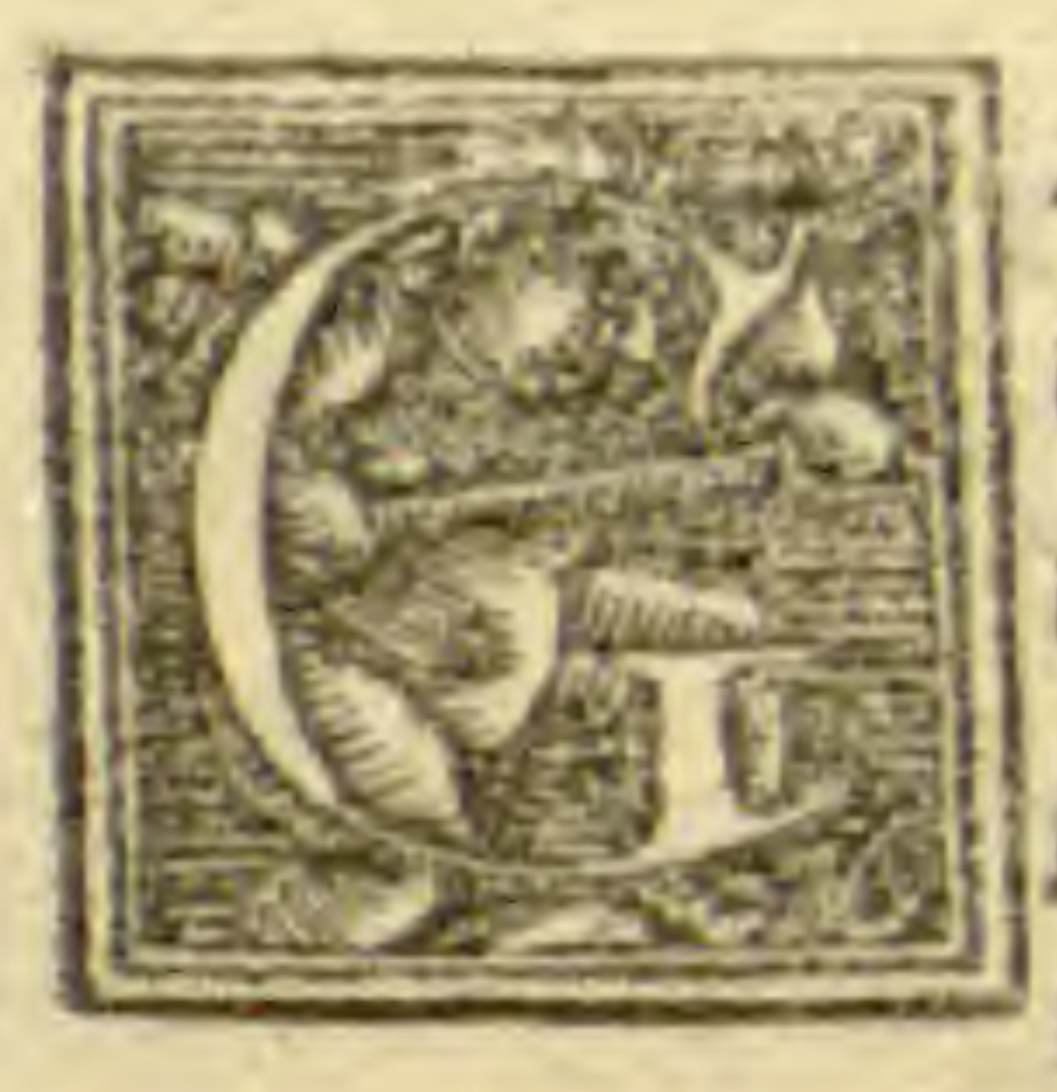

Alinarum, phafianarumq́; ;oua, omni= umoptima funt - Anferü uero o f fru= tocameloruminutilid. NI ultodutépre $=$ ftantiora funtrccentis uetcribus. Atq; ut magis alunt trementia, ita forblia melius dicfcendunt, atg; gutturis afperitates lentunt. Re liqua omria co thu difficilla funt, $\mathcal{F}$ uixtranfmittuntur, cxceptis fuffocatis, que quidem cruda cx garo, uino, $\mathcal{V}^{-}$ olco diluta, , in deplomate modice infpiffantur, ficentm - bonifuccl, $\odot$ cocfu facilia redduntur. Fricta autem infartagme omnium pc $\beta$ ima funt.

\section{DE PEDESTRIBVS}

\section{dnimalibus.}

\section{Suill $x$ carnes.}

$7 \mathrm{X}$ quadrupedibus, futlie carnes omnitund liorum edultorum ualentiftmi funt gencris, quippe que $\mathcal{O}$ fapore $\mathcal{O}$ odore famliarem quandana proprictatem ad bumana corpora refirunt 
P A V L AE G I N T A D E

referunt, utà nomnultis, quiimprudentes, carnes huma nas deguftaucrunt, depreherifum eff . Altmentumuero quod ex ipfis contrahitur $\mathcal{E}$ glutino fum eft, $\odot$ baud farcile disipatur.

\section{Ouilla caro.}

Ouildacaro fuperfluitatibus redüdat, maliq; fuccieft

\section{Capra, Hirci.}

Capre, nutrimentum prebent acre $O$ - mali fucci, peßsumumuerohirci, quippequod non folum mali fucci eft, uerum ctiam concoctonirefiffit.

\section{Bubula caro.}

Bubulacaroatrambilem parit.

\section{Lepores.}

Lepores, cra Ai fuccifunt, $\int e d$ in hoc àbobus ov out $=$ bus uincuntur.

\section{Cerui,}

Cerui, duram, coctuq́; difficilem prabent alumoniam. In fumma uero, recentiora ommia ueterbbus bumi= diora, molliora, $v$ coclu ficilior a funt - Similiter caftra $t a, i j$ squibus integridadhuc tefticulis furt, at $\phi_{j}$ corpulens: tagracilibus.

\section{DE PARTIBVS Animalium,}

Extremx partes. 
RATIONEVICTVS= so Xireme partes neruofe funt, ac pinguedinis carnisq́; expertes. Vnde o glutino fe funt; o alimenti exigui, $d t$; aluum mouent - Exci piuntur aues, propter abundantè ariditatem.

\section{Aures,}

Aures, roftrá; , cartilaginofa funt, $\mathcal{O}$ baud facile concoquuntur.

\section{Lingua.}

Lingua laxa, exanguts, or exigui alimentieft. 2

\section{Glandula:}

Glandule fuaues funt, at $q_{;}$laxe, precipue mamma rum, fed maxime futlle, dum uddelicet lacte replentur, ac ne minoris quidem alımestt f unt, quàm carnes.

\section{Renes. Tefticuli.}

Rencs, tefticuliq́, malifuccifunt, $\sigma$ coelu difficiles. Gallorum faginatorum teftes.

Atqui Gallorum fagindtorum teftes $\mathcal{O}$ fuaues funt, E probum alimentum corpori prebent.

Conira, Taurorum, hircorum, or arictum, coctu. difficilcs, or mali fuccifunt.

\section{Cerebrum.}

Cercbrum pituitofum, craßifucci, tardi tranfitus, coctu difficile, ftomacho alicnum, dtg; naufeofim eft. Vbiuero probe concoctumeft, atis alimentiprebet. ,

spinalis 
PA $\nabla L$ L AEGINETA DE

\section{Spinalis medulla.}

spindlismedulla pinguis eft, 0 fuatior cerebo, fed in reliquis eif tmulis.

\section{Cor,Iecur.}

Cor, iccur $q_{j}$, craßsifuccifunt, coctudifficilia, $\mathcal{O} t \mathbf{r}^{r}$ dimeatus. Atq; fullum bepar celeris melius eft.

\section{Lienis.}

Lienis mali fuccicft, $\mathscr{O}$ atram bilem parit-

\section{Pulmo.}

Pulmo, ut utrisq, laxior eft, ita coctu facilior, fed pis tuojus eft, of minus alit.

\section{Ventriculus, Vterus.}

- Ventriculus, uterus, inteftina, dura funt, uix concos quuntur, atq pituitam gignunt-

\section{Sylueftria animalia.}

In uniuer fum ucro fylueftria animalia, cicuribus $d=$ linentum prebent aridius, minuśs fuperfuitatibus reduridans.

\section{Sanguis.}

Sanguis omis difficulter concoquitur, precipuc $f i$ craffus ac melancholicus oft, uelutibubulus: 


\section{RATIONE: VICTVS 8 i}

\section{Leporinus fanguis.}

Leporino uero fanguini utpote fitan/Simo, precipts us honor eft : Hüc nonnutli cum ipfoiectiore coqucre confueterüt, ac alij cum rellquis quoog;if ceribus. Sunt ctiam qui isuenis porcelli fanguinem edunt. Homerus Home uero, caprinum fang guincm nonnullos commediffe pos ruts. fteritati tradidit:

\section{E L A C TE.}

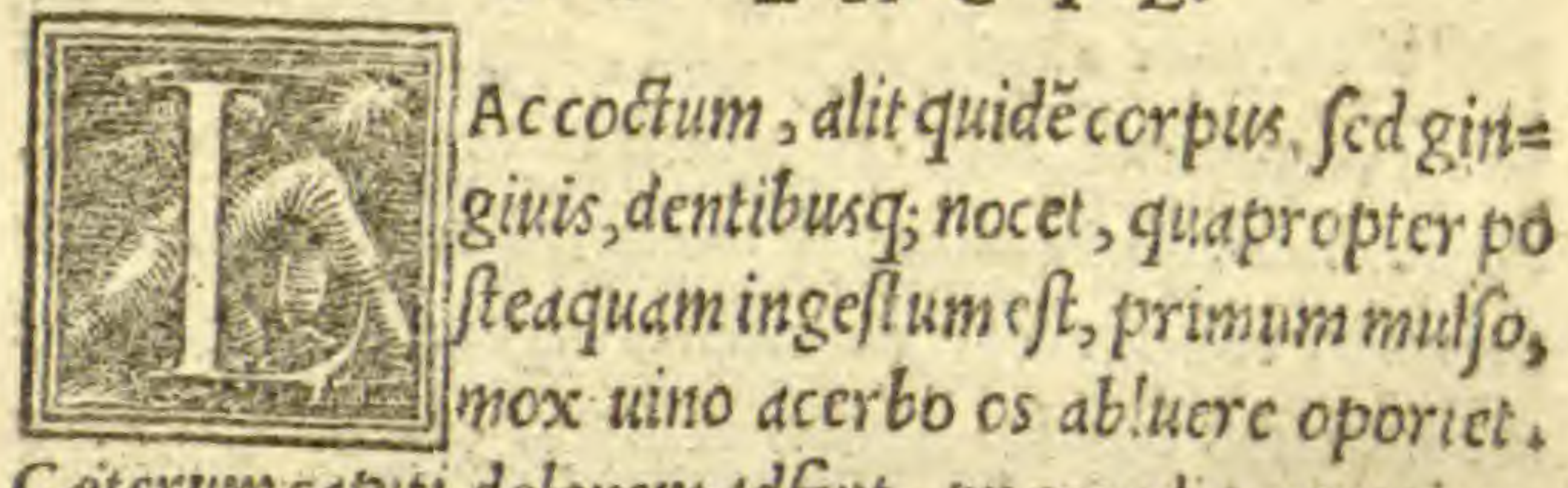
Cetcrum sapiti doloremadfert, precordia uentricke

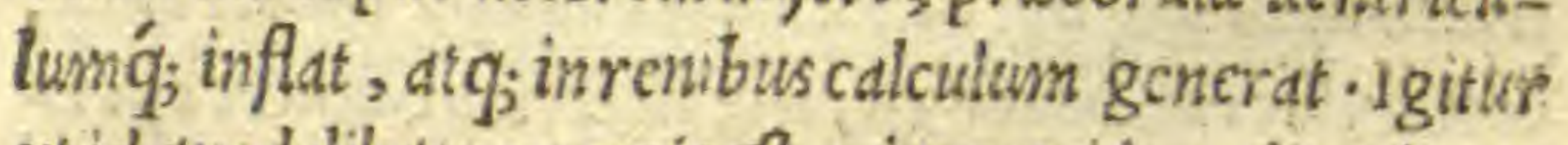
ut id quod dilutum mag is oft, minus quidcmalit, plus ue rocitat aluum, ita ccontrario, crafsius, ualétius quidcm dimentum prabct, , $\mathrm{ed} \mathrm{non} \mathrm{ficiledefcendit.}$

\section{Caprinum lac.}

Porró caprinum lac, mediocris eff fubftantie 3 oull tumcraßioris. Pratered protimus à partutenutus efts tractu ucrotemporiscra ßius, detcrus śg; ffficitur:

\section{QVID O BSER VA N= dum fit, lac potantibus:}

\section{M \\ Qu}


PA $P L$ LE G INETA DE

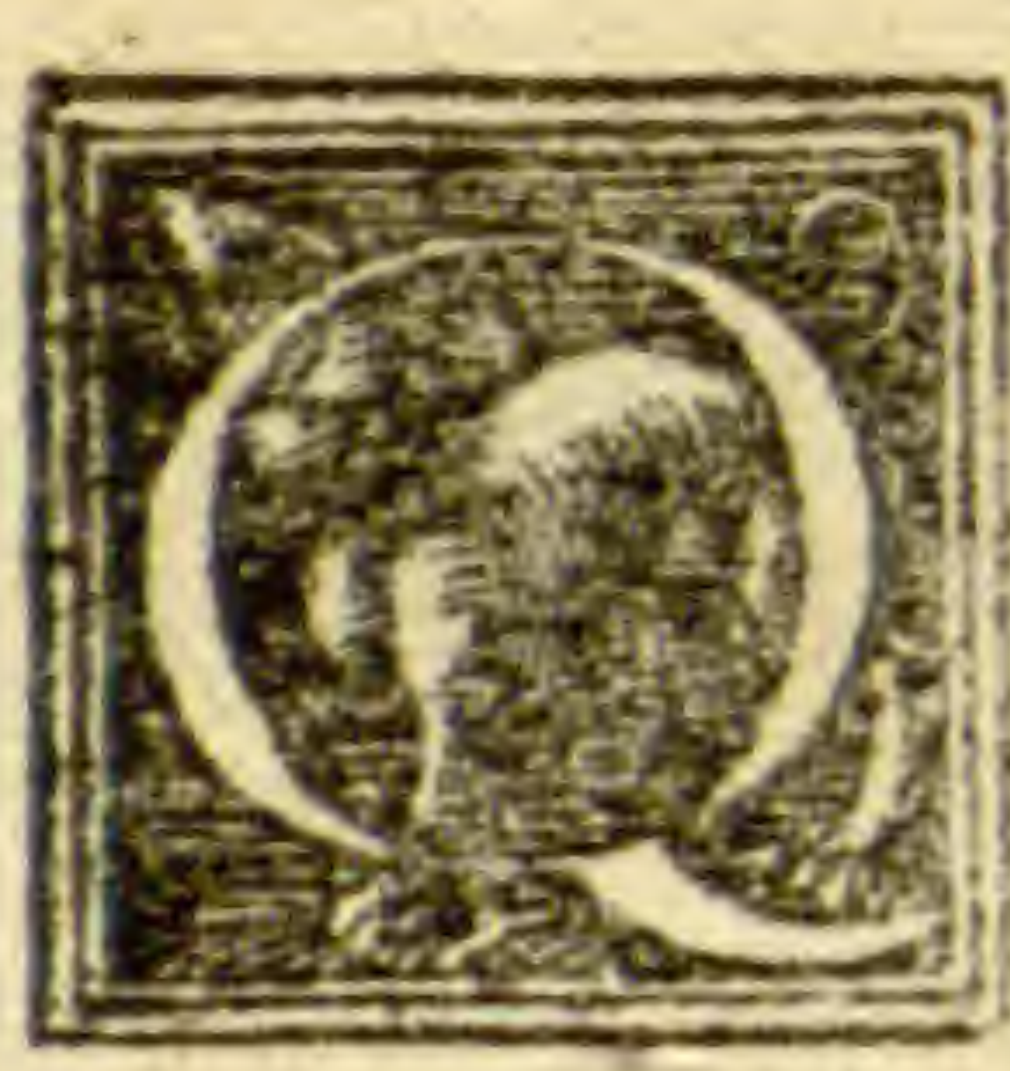

Vi lac potare uoluert, ab alijscibis ab ftnere debet, quoufo; confequatur, ac per inferiora decedat - Igitur preftat ip fum reccns mulctum in malutuno bis bere, deinietunare, at $q$; $t \tilde{u}$ uehementes labores uitare, ace fcit ciil fi quis laboratcrit, ac farus quog; fft leniter deambulare, atq; urgilemiö quic fere. Sane ita facienti, primũ quidem per aluũulubut, ideóf; poft deiectione allud bibendü cft, at $q_{;} ; i$ boc quo $q_{\text {; }}$ ad fecef] um fubierit, allud. In primis enim uttliter purg.t, nõ tta multü quidé uniuer fe molis, fed ea duntaxat que in uentre continentur, mox uenas ingreditur, at $q$; opti me nutriens haudquaquam deijcitur. In billofis uero fluxiontbusatg; ubi teetriculum eliquart timuertmus, coctumlac cxhbbendü eft, atq; fic ip fum coquere opor=

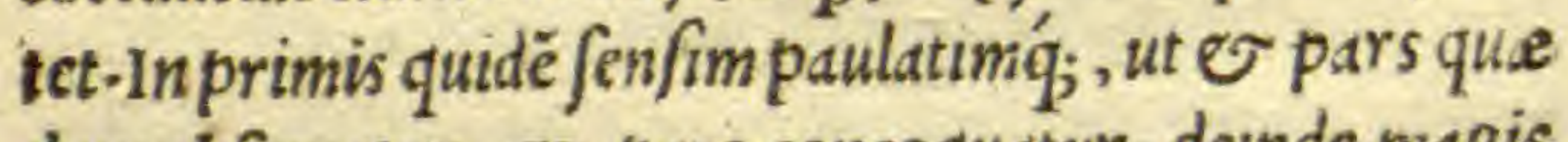
damabfumatur, $\mathcal{O}$ pars concoquatur, dende magis atq; magiscoquere. Cauendum uero ut neq; aduratur, neq; incafcum coaguletur, id q $^{\prime}$ optime fiet, fileniter $i=$ pfum coxerimus, o mifcendoid quod ab fcefferit, un= dig; detraxerimus: I I itur ferula poltta, tenuiq; ; mifcere oportet, 0 fi quid in labijs aule firuet, id /pongla pur gare. Hocentm fepenumeroreliquum lac corrumpit Ergocoquendum eft lac ut diximus, donec equabiltter craßus, $\mathcal{O}$ cridolacie dulciuscuadat. Preterea quod inlacte tenue ferofun'śg; eftconfumtur, ficalcult mari ni cxigne candentes in ipfum demittantur, id q́; aluo fiuenti 
RATIONE VICTVS, 8 B flucnti, maxime fi bilio fa fuerint excrementa sximic prodeft.

\section{E $L A$ A C $T$ T Schifto.}

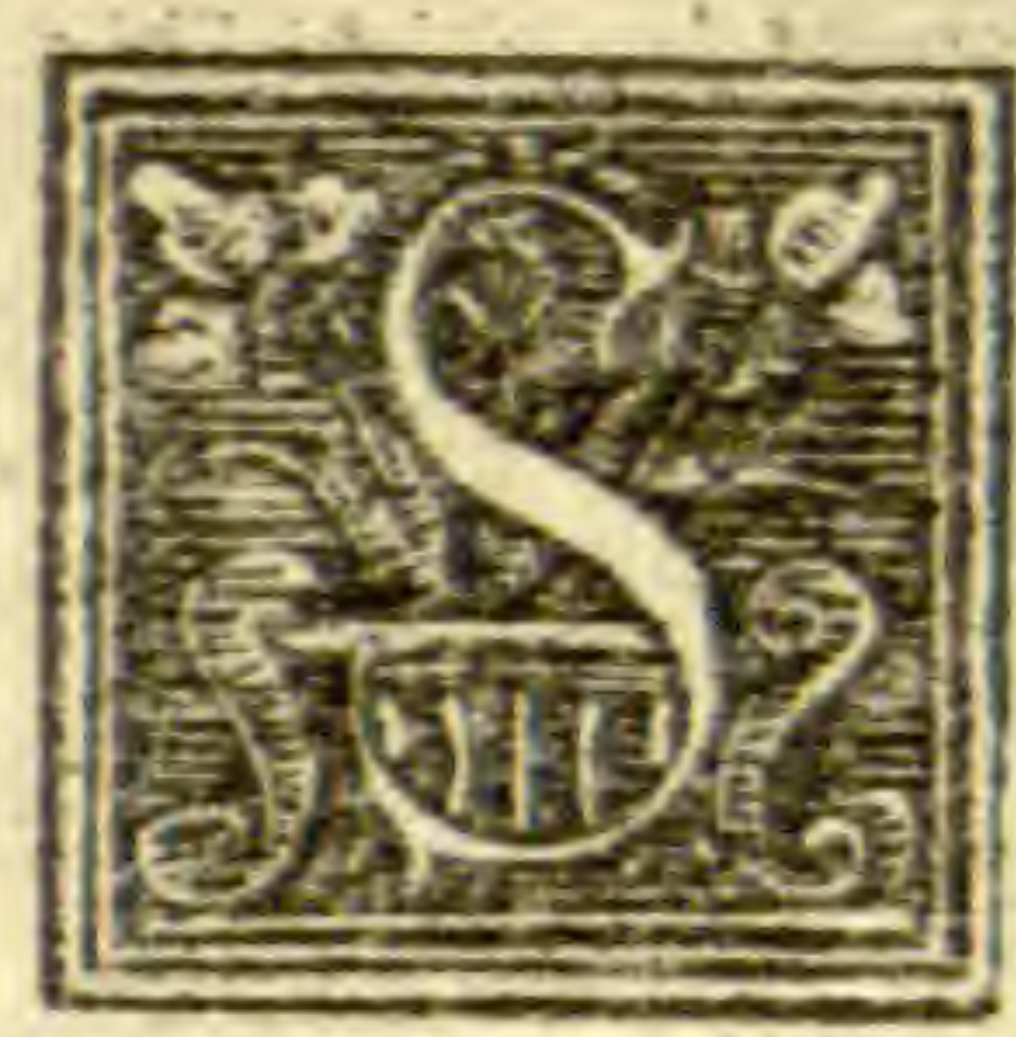
Erumlactis à cafeo difcernitur, fiuche menter ad multum fumum coquatur. Deinde exacte uel per linteum, uel per pannucolctur. Mifcetur at ferofe par th, uel mellis uel oximethis, uel falis mo dicum, at $q_{j}$ ut aluum fubducat datur, adultis quidem ad: fextarios duos, iunioribus iverohand minus fextarios Quoduero marinorum calculorum, aut ferriexigne candentis demißione incraffatum eft lac, dyfenteriz, $\mathcal{O}-$ alui proflutio haud mediocre prefidium eft.

\section{DE C A S E O.}

A feus omnis acris eft, fitim excitans, co=
culu difficilis, mali fuccl, calculum gigneus. Innocentior veroe $t$, quirecens eff, laxus; motlis, fuauis 0 modico fale conditus, qu $i$ uero allter efficitur peßsimuseft.

\section{DE PISCI B V S}

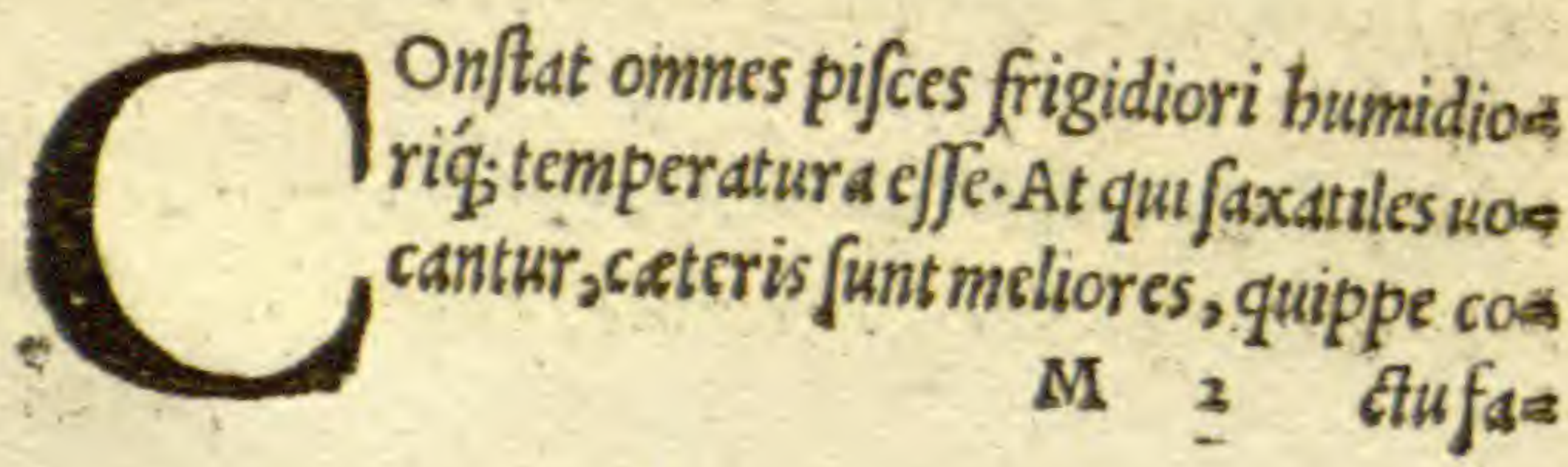




\section{PA $V$ L,$A E G I N E T A D E$}

Etu faciles [unt, boni fucci, atqg, modice bumectant, nit/i dura fuerti eiscaro. Non faxatilium autem, in pelago degentes, multodifcrimine differunt abi is, qui inlima nutriuntur, aut qua fumind irruunt, fedomnium pe ßi ma qui uel inftagnis uel paludibus aluntur.

\section{Capito, Lupus.}

Atparticulatin capito quiden, fin pelago degue= rit, modice fuanis eft, fanguinem uero prebet $\odot$ te= nutore 0 imbecillorè Huichaud abfimilis lupuseft.

\section{Mullus.}

Acin equore quoq; mullusuer fatur, ceteris picci= bus $\odot$ durtor $\mathcal{E}$ fraglior, coctufaclis, menfis idone us, predulcis, punguedinis expers.

$$
\underset{\substack{\text { Q } \\ \text { protegmine } f \text {. }}}{\text {. }}
$$

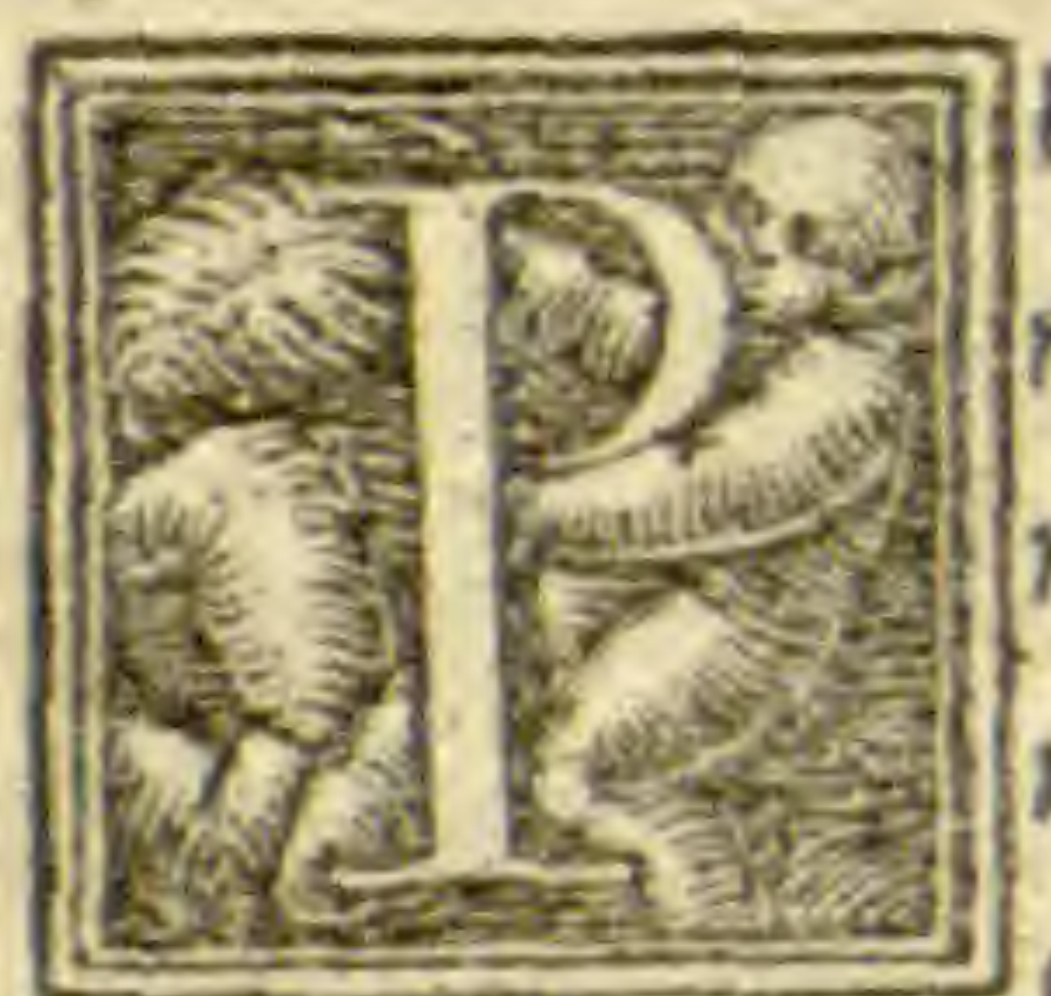

If cium genera que tefta integuntur, $0=$ mnino falf fum crudum $q^{\prime}$, humorem $p d=$ riunt. Horum oftrea quiden mollifi= macarnefunt, o magis aluum ducunt, Chame nero 0 purpure o ungues - F Rondyli, $\sigma$ buccina $\sigma$ cochlce, atque hutufmodi alia dura, ac que cruftata quog; nomiatur, ut Grams marij, Paguri, Cancri, Locufle, Sqquille, mé/is quidem 


\section{RATIONE VICTVS.}

conueniunt, fedcoctu difficilia funt, dic fi $\int e p e$ in $d q u d$ dulci clixentur, aluum aftringunt. I us omnium que te $=$ ftis concluduntur aluum ducit, hanc ob caufam terre= fires quoogcocblece uentrem foluunt. At uero dure car nis e coct $u$ difficilis funt, que licet nutriat, mali td= men fuccieft, quapropter nönulli tus cum garo 0 oleo condiunt, at q; fic uentrem fubducunt.

Echint modice rcfrigerant, parum alunt, atque uris ham cient.

D E M O L L I B V S.

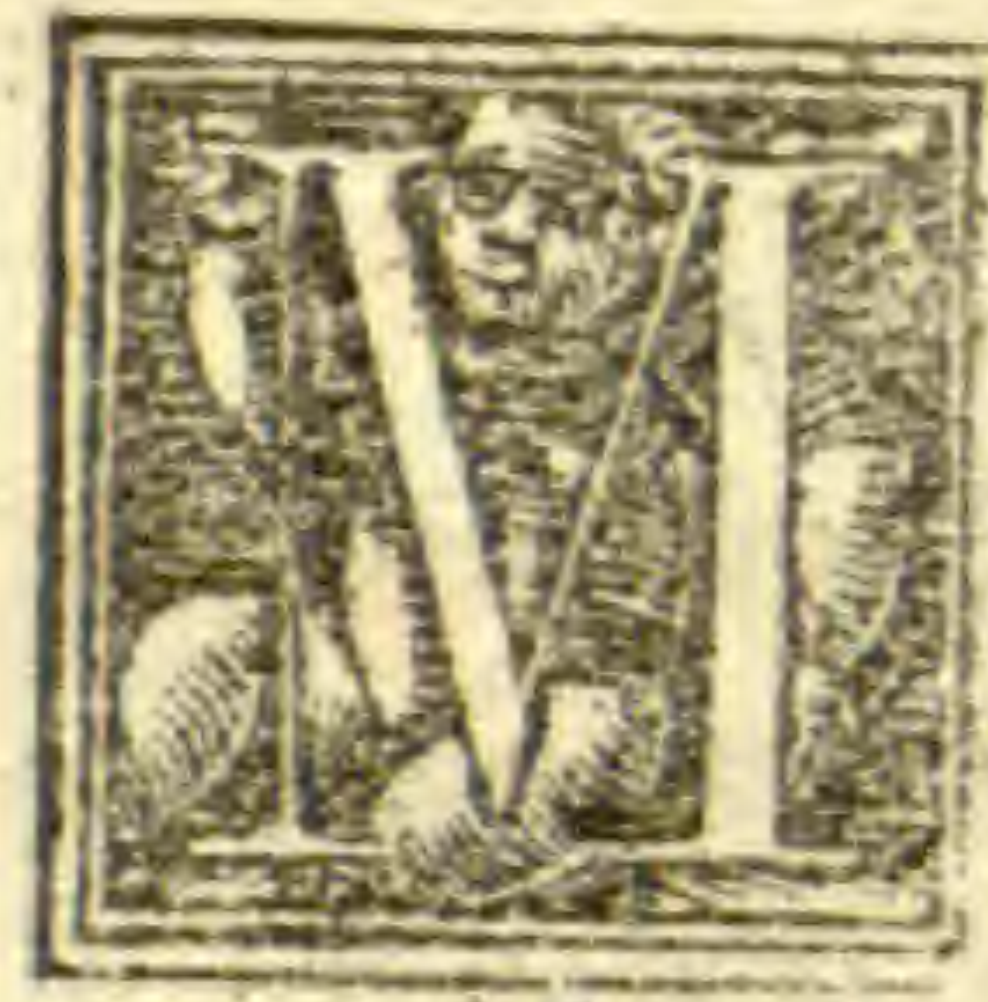

Olles pifces, ut polypi,fepia, lolligenes, durifunt, coctionig; rcnituntur, prefer timpolypi, at g; falfum quodammodo ha tent humorem siucro concoctione uin cantur, baud parum alimenti corpori prebent.

\section{DE CARTILAGINETS.}

T. $\mathrm{X}$ gencre Cartilagineo, torpedines, $p$ aftind $=$ ca, molle atq; predulcicarne conftant, que et celcrtter fubit, $\odot$ facle concoquitur, alit $\hat{q}_{;}$ Rata, letirate; fquatine, o reliqua baiufce modigenera, duriora coctúq; diff cilora funt, $\mathcal{O}$ plus aliment prabent. Atg; in toium, dure carnis anmalia, baud facile concoquuntur, fcd $\mathcal{O}$ ualens $\mathcal{O}$ folidum ald nentum prebeni.

M 3 De Cetaceis 


\section{DE CE TACEIS.}

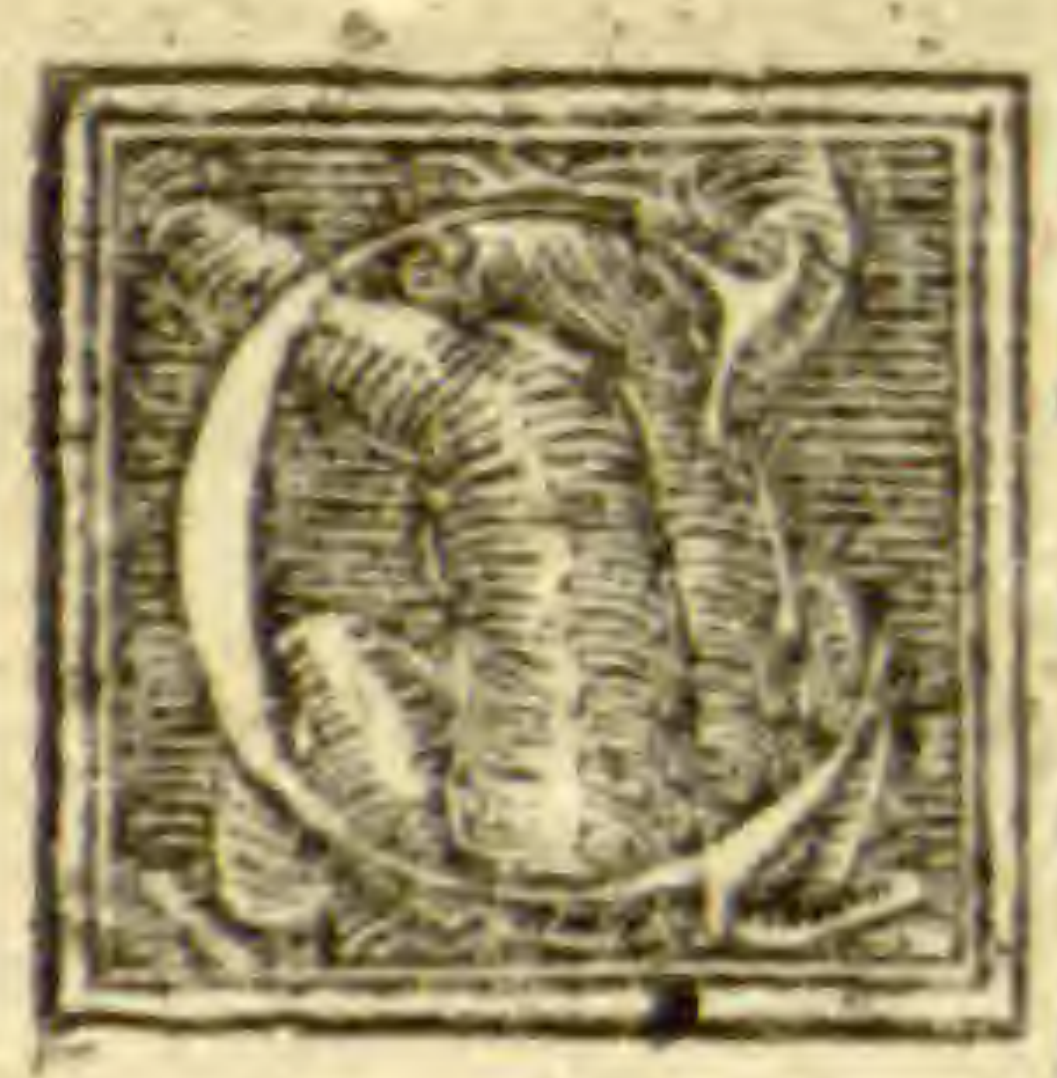

Etaci, ut balena, uitulus marinus, li= bella, delphini, $\mathcal{E}$ thunnipragrandes, dure o coetu diff cilis funt carnis, dC craßifucci. Sale autem conditta modera tiora redduntur, atq;alijs pifcibushu= midiora-Superfluttaibusmagis abundant, $\mathcal{E}$ ad per $=$ turbandium proniora funt, Salfamentorum differétia, pifctum ex quibus conftant fequiuntur difcrimina.

\section{E E V I I N I}

\section{potentia.}

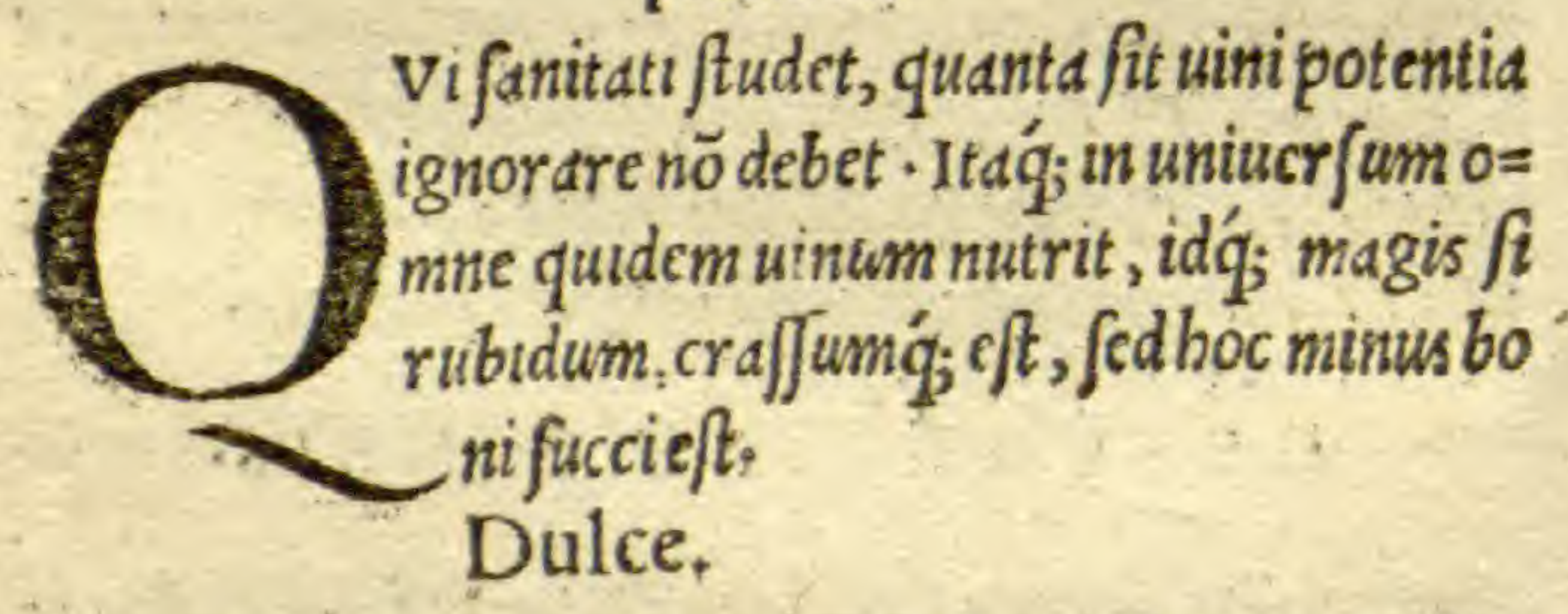

Dulccuero, quamuis nutriat fomachotamenalies numeft.

\section{Acerbum.}

Acerbum ftomac bo quidem gratum, fed non facile toranfmettutur, ac motnus alit, td'q; multo monus if fucrit, album.

\section{Fuluum.}

Fuluno nuitum atind melius cf?. 
RATIONE VICTVS. 84 Vinum Vetus.

Vetus uinum recenticalidius, ficciusq́; eft. Atque in fumma uini potentia bac eft.

In totum uero uinum calorem noftrum excitat, un= de $v$ coctio melior, e fanguinis utilior fit. Cibum, uts $=$ pote facile penetrans in omnes partes deducit, ob quàm caufam attenuatis quoq; ut morbl, bonum habttum re= ftituit, cibiappetentiam crigut, pituitam cxienuat, bi $=$ lem p.rurinas expurgat, colorem emendat, animum hi lariorem tucundum q́; reddit, corporis robur aluget. At que he funt utni modice epoti commoditates. Immodi= ce uero ingeftum, contrarta efficit, hinctemulentor um euerfiones, dementie, fopores $q$; peruenunt-Qu upro= ptercaliéda eft immodcrata uimi potatio, quamuis $f \mathbf{e x}$ longis temporum interuallis liberalie er bibatur, com= moditate non carcat, quando $\dot{q}_{\text {; }}$ urinds abunde moust, ac fudorcs profundit. At mox affumpto mulfoipfum cuomere fatius eft, ut ne parua quidem noxe ueftigium relinquat. 1 gitur potationi operam daturus alio ctbo non admodum fi replere debet, atque intcr bibcndum Braßtcamclixamedere, of bellaria, prefertim que ex angygdalis conftant, deguftarc $\cdot$ Hec enim capitis dolo= remlcuant, neq; ullam uomentibus moleftiam offeruntAc macerat quoq; abfynthij cremor ante potationem fumptus, biud medocrice auxilutur, ut ne altud qui= demunuentripoßit crapule magis intmicum.

$$
\text { M } 4 \text { Cnod }
$$




\section{P A V I. AE GIN E T A. DE}

owod fi quempiam mordeat uinum, frig:dam aqudn? Wibere debet. Poffero die abfyntbij cremorem fumere, ambulare, fricari, lauacrumingredi, atg; fubinde exis: guociborefici.

$$
\begin{gathered}
\text { DE M ELLE ET } \\
\text { MLUIJO. }
\end{gathered}
$$

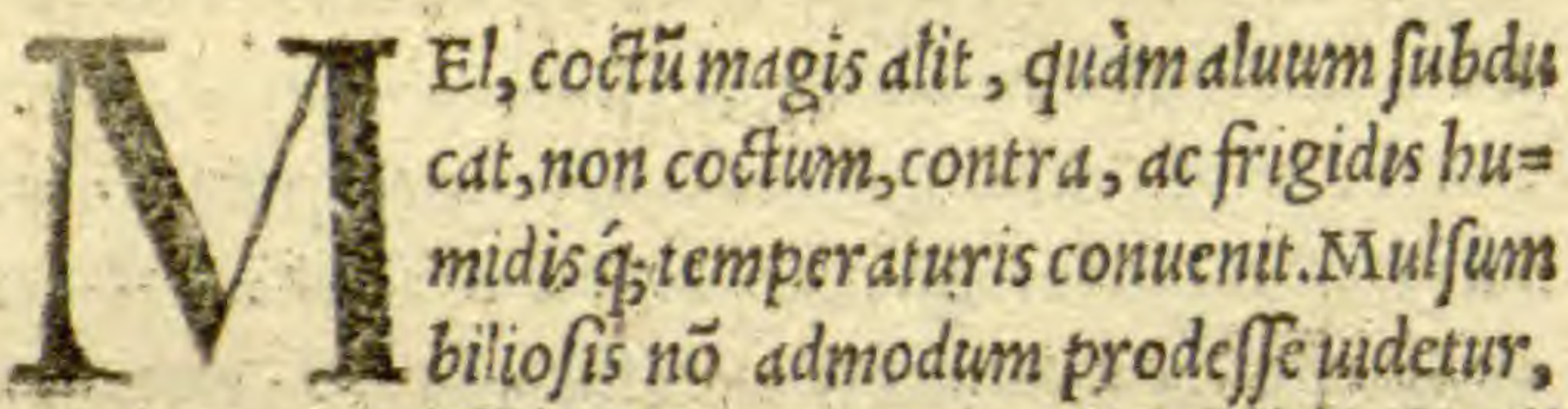
guippe quad if fis in bilem uertitur : Igitur hisufmodi naturis oportctipfum magis aquo fum exhibere, at qui bus uf ccra quoquomodo afficiuntur, is alienum eft.

Caterumbocmodomul lum preparatur, octuplum sque melliadijctitur, ac donec pumare defieritcoqui= tur, fpumam ucrofimul atg; orta cft, ingiter auferre pportete.

\section{DE $S O M N O$ :}

Y Vpereft poft omnia qua de uiçus ratione dixis mus, ut dc fomno quoq, diffor amus, quippequi $1)$ omnium ultimus magnda ex parte admittitur. igitur formusu, ut formula quadam loq̣uamur, ef t dnimalum nirium quies, ab ut hi humorccercbrum madefaciente proseniens. Hunc q̧uirediecape ßit, mul ta confequitur commoda Cibos entm conficit, bumores digerit,dolorcs $f$ cdat, fattigationes folatur, rigidare $=$ laxals 


\section{RATIONE VICTVS 8 F}

laxat, animi egritudines oblixione delet, mentisinfani atn corrigut . T cmpus fomno accommodatifumum eft, poft cibum, interdiu uero faclus, haud omnibusidone= us eft, id enim tempus quo dormiunt alcqui, hatud fatis of , ut in eo cibi perficte conficlantur . Intercifacnim ante fuam horsm concodtonc, per meridicm exurgen= tes fepenumero acidum ructant, flatibus implentur. Nonnullifuctuatione quoq; moleffantur, nifítuelexcō fuetudine, ueiquta fatis dormierunt, huut fcemodicda fus effugiant.

Noclurnosutem fomno baud alius melior eft, fiqui= dem nox fuapte natura tum bumore, tum tranquillitate fomnum alliciens, coctionem perfectam cfflcit .

Praterea post notturnos fomnos, magna exparte ad excrementadeijciendafeftunamus, Temporis au tem fomno debits terminus, ex alimenterum perfecta concoGione funitur, quam tum cxrufibus, tume cxuers tris contraction deprehendemus. Poft hanc, cxcitd= to non inuties eff, utpote, que concc flonis sxcremen: ta ad feceffum agt.

D E V I G.I I IA.

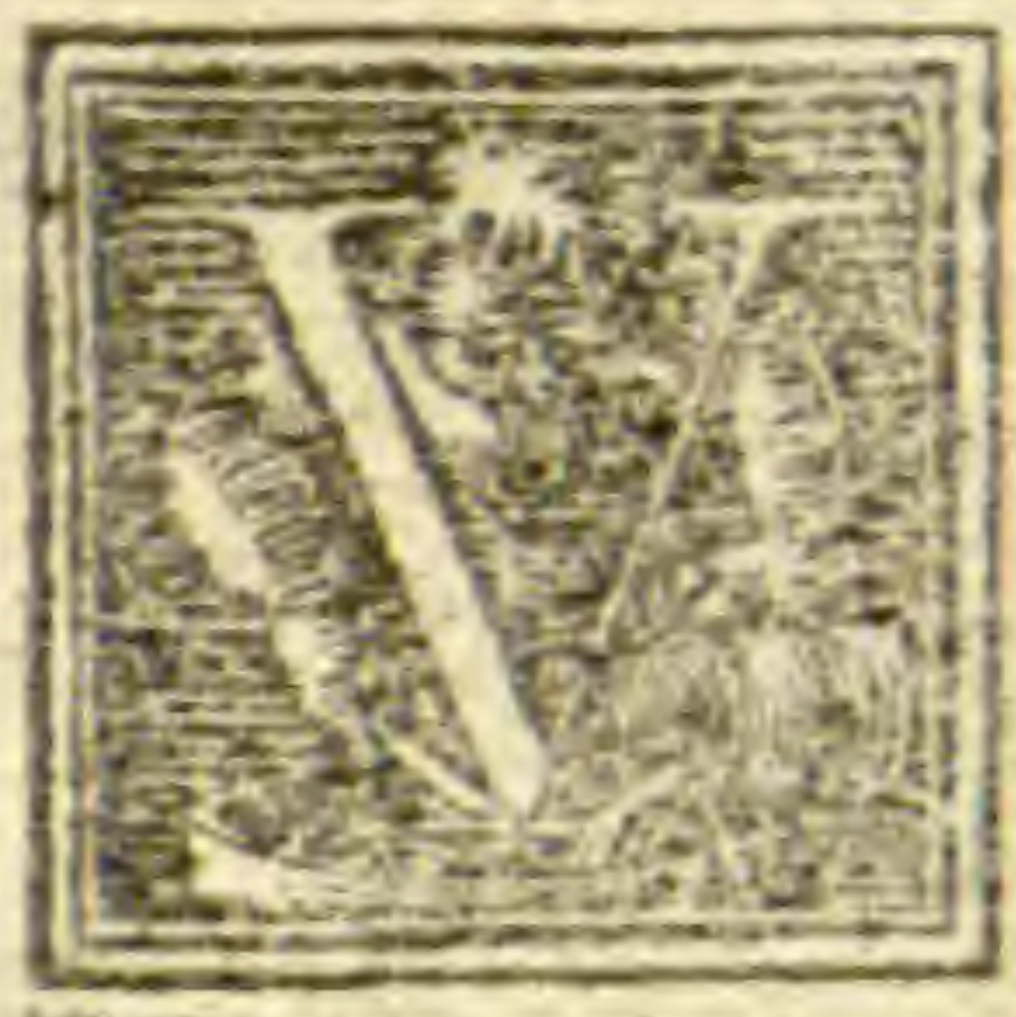

Iglidrum, que iam agyotantes, dut exdolorc, ant fobri, aut alio quouts ac= cid nie inferfant, curationem, in libro defibribus perfequenar.

un prefentio uero de his, wigulijs Ms 


\section{$\therefore$ P A L $A E G I N E T A D E$}

dumitaxat dicendum eft, que fanos interdummale aff $=$ ciunt. Igitur fi ucl ob moefticiam, Hel follicitudinem, aut aliam antmi agrttudinem quis ugilauerit. Imprimis mororem, quoad fieripoteft, fugabimus. Deinde $\rho_{0}=$ norum fuauitate, cogitatio abigenda eft, id quod non= nulliper lenes aque fonos efficcre molluntur, quienin fic demulcentur, facile fomnum fubeunt - Ac balncum quog; poti ßimum in ue $\beta$ era, cumbene concoxcrit, in= grediantur. Ciburq́; bumectantem affumant, ut ladeu cas, allosq́; buiufcemodi. Nigri quoq; papauerisuiridia folla obfonijs interdüadijciant, pifces coctu fácles edät, Vinumtenue, non antiquum, libcraliter bibant. $\mathrm{C} u=$ bilepetturi; rofaceocaput ungant, aut oleo, in quo pas paueris capita, aut mandragore decota fucrint. Ane= thinum etiamnum recens, fommiferum eft. Nouimus nonnullos polt farigationem fubito quicuiffe, ac fubin= - de dorminiffe. Id q́; ex modica etiam Vencre accidere fo let - Alì infrigidatocapite uel pedibus, fomno ficile cor ripiuntur. Quodfiobcibiuclmulitiudinem, uel mali= ciam, grauato ftomacho, alque uigul re cogantur, ntm o boc accidi]fecompertum habemis, qucridmodum alij rurfus preter confuetudinem dut tetunantes, aut exiguumabum fumentes, dormin: nequeunt, perpen

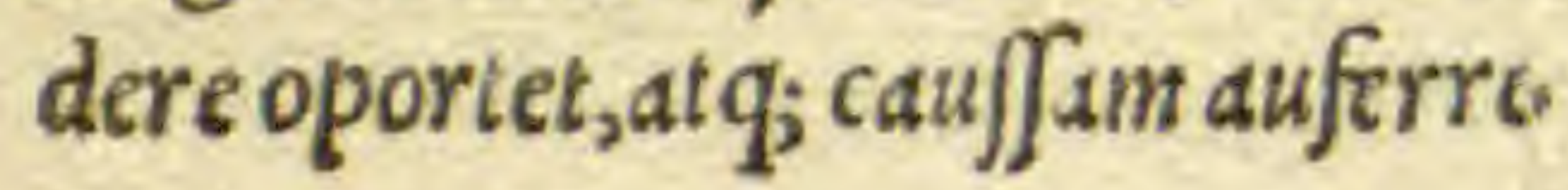

\section{DE SOPJRE}




\section{RATIONE VICTVS: E6}

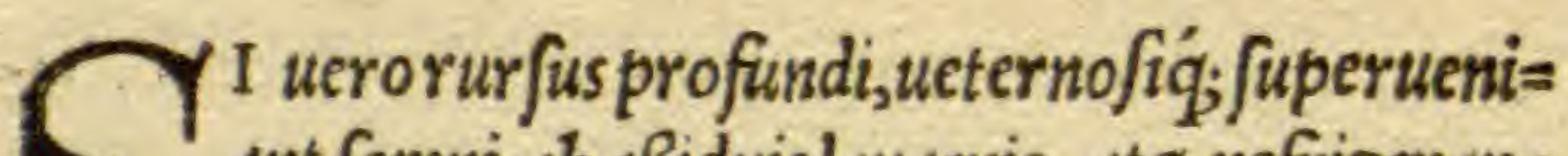
ant fomni, ab a ßiduis laudcris, atq; refrigeran= 1 tc unctione abftinere oportet. Apophlegmatif $=$ mis uero uti, atq; omninouictus rationem cali = diorem, aridiorem $q$, o cxiguum alimentum exhibere, quandoquiděbuiufcemodiaffs clus, à frigidiore, humidiorég;ma teriacerebrum irrigante,

magna ex parte accidit.

$\therefore$

PAVLI AEGINETAE DE RATIONE VICTVS. FINIS.

ARGENTORATI EXCVDEBAT GEORGIVS VLRICHER A NDLANVS, ANNO M. D. $\mathrm{XXXI}$. Menfe Sept.

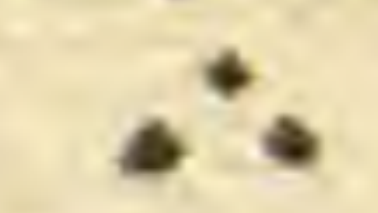

